\title{
Heavy ion collisions and neutron stars: dynamics and thermodynamics of QCD matter
}

\author{
Dissertation \\ zur Erlangung des Doktorgrades \\ der Naturwissenschaften
}

\author{
vorgelegt beim Fachbereich Physik \\ der Johann Wolfgang Goethe-Universität \\ in Frankfurt am Main
}

\author{
von \\ Anton Motornenko \\ aus Kiew, Ukraine
}

Frankfurt am Main 2021

D30 
vom Fachbereich Physik der

Johann Wolfgang Goethe-Universität als Dissertation angenommen.

Dekan: Prof. Dr. Harald Appelshauser

Gutachter: Prof. Dr. Horst Stöcker

Prof. Dr. Marcus Bleicher

Datum der Disputation: 19.07.2021 


\section{Contents}

Zusammenfassung iii

I Introduction $\quad \mathbf{1}$

I.1 Symmetries in fundamental physics . . . . . . . . . . . 6

II Hadron yields and fluctuations in heavy ion collisions: thermal and transport model calculations 13

II.1 Canonical Ensemble Hadron Resonance Gas . . . . . . . . . . 14

II.2 Hadron yields in the UrQMD model . . . . . . . . . . . . . 18

II.3 Comparison with the experimental data . . . . . . . . . . 23

III Modeling the hadronic phase of heavy ion collisions by an expanding hadron gas 29

III.1 Hadronic phase in heavy ion collisions . . . . . . . . . . . . . . 30

III.2 Partial chemical equilibrium in Hadron Resonance Gas . . . . 31

III.3 Kinetic freeze-out temperature from yields of short-lived resonances 34

IV Repulsive interactions between baryons in a hadron gas

IV.1 Classical excluded-volume model . . . . . . . . . . . . . . . . . 44

IV.2 Beth-Uhlenbeck approach . . . . . . . . . . . . . . . . 46

IV.3 Estimating the hadron repulsion from the lattice QCD data . . 54

V A unified approach for QCD matter: Chiral Mean Field model

V.1 Chiral Mean Field model . . . . . . . . . . . . . . 60 
V.2 Constraining the CMF model to the lattice data . . . . . . 66

V.3 The CMF model phase diagram . . . . . . . . . . . . . . 69

V.4 Taylor expansion at large densities and the role of HRG particle list . . . . . . . . . . . . . . . . . . 75

V.5 Application to heavy-ion collisions . . . . . . . . . . 76

V.6 Application to neutron stars . . . . . . . . . . . . . . . 82

VI Repulsive properties of hadrons in lattice QCD data and neutron stars $\quad 89$

VI.1 Lattice data comparison . . . . . . . . . . . . . . . . . 90

VI.2 Consequences of the modified excluded volumes . . . . . . . . 94

$\begin{array}{ll}\text { VII Summary and Outlook } & 107\end{array}$

$\begin{array}{ll}\text { Bibliography } & 111\end{array}$

$\begin{array}{ll}\text { Acknowledgments } & 133\end{array}$

$\begin{array}{ll}\text { Curriculum Vitae } & 134\end{array}$ 


\section{Zusammenfassung}

Diese Arbeit basiert auf folgenden Publikationen:

- V. Vovchenko, A. Motornenko, M. I. Gorenstein and H. Stoecker, "BethUhlenbeck approach for repulsive interactions between baryons in a hadron gas," Phys. Rev. C 97, no.3, 035202 (2018) [1]

- A. Motornenko, V. Vovchenko, J. Steinheimer, S. Schramm and H. Stoecker, "QCD at high density: Equation of state for nuclear collisions and neutron stars," Nucl. Phys. A 982, 891-894 (2019) [2]

- A. Motornenko, V. V. Begun, V. Vovchenko, M. I. Gorenstein and H. Stoecker, "Hadron yields and fluctuations at energies available at the CERN Super Proton Synchrotron: System-size dependence from $\mathrm{Pb}+\mathrm{Pb}$ to $p+p$ collisions," Phys. Rev. C 99, no.3, 034909 (2019) [3]

- M. Hanauske, J. Steinheimer, A. Motornenko, V. Vovchenko, L. Bovard, E. R. Most, L. J. Papenfort, S. Schramm and H. Stöcker, "Neutron Star Mergers: Probing the EoS of Hot, Dense Matter by Gravitational Waves," Particles 2, no.1, 44-56 (2019) [4]

- A. Motornenko, J. Steinheimer, V. Vovchenko, S. Schramm and H. Stoecker, "Equation of state for hot QCD and compact stars from a mean field approach," Phys. Rev. C 101, no.3, 034904 (2020) [5]

- A. Motornenko, J. Steinheimer, V. Vovchenko, S. Schramm and H. Stoecker, "Matter And Gravitation In Collisions of heavy ions and neutron stars: equation of state," PoS CORFU2018, 150 (2019) [6]

- A. Motornenko, V. Vovchenko, C. Greiner and H. Stoecker, "Kinetic freezeout temperature from yields of short-lived resonances," Phys. Rev. C 102, no.2, 024909 (2020) [7] 
- A. Motornenko, J. Steinheimer, V. Vovchenko, S. Schramm and H. Stoecker, "QCD equation of state at vanishing and high baryon density: Chiral Mean Field model," Nucl. Phys. A 1005, 121836 (2021) [8]

- A. Motornenko, S. Pal, A. Bhattacharyya, J. Steinheimer and H. Stoecker, "Repulsive properties of hadrons in lattice QCD data and neutron stars," [arXiv:2009.10848 [hep-ph]] [9]

Die Eigenschaften von stark wechselwirkender Materie und die Zustände, in denen sie auftreten kann, sind ein schwer fassbares Problem der modernen Quantenphysik. Die Quanten-Chromo-Dynamik (QCD), die fundamentale Quantentheorie der starken Wechselwirkung, gehört zu den am besten etablierten Theorien. Die Theorie der QCD ist nicht nur notwendig, um die Stabilität von Atomkernen zu beschreiben, sondern sie ist auch für die Entstehung von etwa 99\% der sichtbaren Masse im Universum verantwortlich (der Higgs-Mechanismus ist nur für das restliche $1 \%$ verantwortlich). Aufgrund der Komplexität der Wechselwirkungen in der Theorie sind direkte Berechnungen grundsätzlich nicht möglich. Daher ist die Verwendung von phänomenologischen Modellen zur Beschreibung von QCDMaterie in der wissenschaftlichen Gemeinschaft gut akzeptiert. Die sich daraus ergebende QCD-Phänomenologie deutet auf ein ziemlich reichhaltiges Phasendiagramm der QCD-Materie bei hohen Temperaturen und Dichten mit der möglichen Existenz extremer Materiezustände an, jedoch wurde noch kein wirklicher Konsensus über die Struktur des Phasendiagramms erreicht. Weltweit werden erhebliche Anstrengungen zur experimentellen Untersuchung des QCD-Phasendiagramms mit Hilfe von Hochenergie-Atomkern-Experimenten an Collider-Anlagen unternommen. Die Vorhersage eines Phasenübergangs zu einem neuen Zustand der Materie, der mit dem Bruch fundamentaler Symmetrien verbunden ist, weckt großes wissenschaftliches Interesse. Nach der Entdeckung der kosmischen Kollisionen zweier Neutronensterne, der dichtesten bekannten Objekte, deren Gravitationskollaps nur durch die Wechselwirkungen von Elementarteilchen verhindert wird, wurde vorgeschlagen, die von einem solchen Ereignis emittierten Gravitationswellen zu nutzen, um die Eigenschaften von QCD-Materie zu untersuchen.

Diese Doktorarbeit befasst sich mit der Phänomenologie der QCD-Materie, ihren Aspekten bei Schwerionenkollisionen und in Neutronensternen. Die erste Hälfte der Arbeit, die aus den Kapiteln II, III und IV besteht, konzentriert sich auf die hadronische Phase der QCD-Materie. Ein Schwerpunkt liegt darauf, wie 
sich die hadronische Phase bei Schwerionenkollisionen zeigt und wie ihre Dynamik simuliert werden kann.

Verschiedene Phasen von QCD-Materie, die bei Schwerionenkollisionen erzeugt werden, können nicht direkt nachgewiesen werden, da die Materie die experimentellen Detektoren nur in der hadronischen Phase erreicht. Es wird angenommen, dass sich das Auftreten des Übergangs zu einer anderen Phase in den Eigenschaften der gemessenen Hadronenzhlen widerspiegelt. Die gemessenen Fluktuationen der Hadronenzahl und einige spezielle Kombinationen von Hadronenmultiplizität, wie das Verhältnis $K^{+} / \pi^{+}$, werden als die bekanntesten angesehen. Diese Observablen reagieren jedoch auch empfindlich auf dynamische Effekte der Kollision, wie die Kollisionsgeometrie und die begrenzte Akzeptanz des experimentellen Detektors. In Kapitel II dieser Arbeit wurden mikroskopische Transportmodellsimulationen für SPS-Energien durchgeführt und mit thermischen Modellvorhersagen verglichen, um einen Referenzwert für das Verhältnis $K^{+} / \pi^{+}$und die Fluktuationen der Partikelmultiplizität zu erfahren. Das UrQMD-Transportmodell und das Hadron Resonance Gas (HRG) -Modell wurden verwendet, daher waren die betrachteten Szenarien a priori ohne Übergang zu einer anderen Phase der QCD. Um den experimentellen Versuchsaufbau zu imitieren, wurden die Zentralitätsauswahl und die Detektorakzeptanz der NA61/SHINE-Kollaboration in den Simulationen reproduziert. Ein Vergleich mit verfügbaren experimentellen Daten wurde durchgeführt. Es wird vorgeschlagen, ein Zentralitätsauswahlverfahren in p+pReaktionen zu implementieren, um die dynamischen Eigenschaften der Kollisionen zu verdeutlichen.

Statistische Modelle mit nur wenigen thermodynamischen Parametern liefern eine überraschend gute Beschreibung der Hadron Multiplizitäten aus dem System mit komplizierten Nichtgleichgewichts-Dynamiken und Wechselwirkungen. Die thermische Modellanalyse ermöglicht die Abbildung des chemischen Ausfrierens von Schwerionenkollisionen auf das QCD-Phasendiagramm. Das chemische Ausfrieren des Systems, der Moment, in dem die chemische Zusammensetzung des Systems festgelegt wird, ist jedoch nicht die letzte Etappe seiner Entwicklung. Das System dehnt sich weiter aus und kühlt ab, bis das sogenannte kinetische Ausfrieren auftritt. Ab diesem Punkt kann das kinetische Gleichgewicht nicht mehr aufrechterhalten werden. Die Temperatur des kinetischen Ausfrierens wird häufig durch Fits an die $p_{T}$-Spektren stabiler Hadronen extrahiert. Dieses Verfahren hängt von den Annahmen bezüglich des Strömungsgeschwindigkeit- 
sprofils und der Ausfrierhypersurfläche ab. In Kapitel III dieser Arbeit wird eine Methode zur Bestimmung der kinetischen Ausfriertemperatur bei Schwerionenkollisionen aus der gemessenen Multiplizität kurzlebiger Resonanzen vorgestellt. Die Methode basiert auf einer Beschreibung von Schwerionenkollisionen durch ein sich isentropisch ausdehnen Hadron-Resonanz-Gas im partiellen chemischen Gleichgewicht. Die Expansion wird zwischen dem chemischen und dem kinetischen Ausfrieren modelliert; in dieser Phase werden die Multiplizität vieler kurzlebiger Resonanzen unterdrückt. Die Werte von $T_{\text {kin }}$ und $T_{\text {ch }}$ werden für verschiedene Zentralitäten in $\mathrm{Pb}-\mathrm{Pb}-$ Kollisionen bei $\sqrt{s_{N N}}=2.76 \mathrm{TeV}$ durch Anpassung von vielen sowohl stabiler Hadronen als auch kurzlebiger Resonanzen wie $\rho^{0}$ und $\mathrm{K}^{* 0}$, die durch die ALICE Kollaboration bestimmt wurden. Dieses Verfahren ermöglicht es, die kinetische Ausfriertemperatur aus den gemessenen Hadronenund Resonanzmultiplizität zu extrahieren, unabhängig von Annahmen über das Strömungsgeschwindigkeitsprofil und die Ausfrierhyperfläche.

Nachdem die dynamischen Aspekte von Schwerionenkollisionen und die bei diesen Kollisionen verfügbaren Temperaturen abgeschätzt wurden, können die Eigenschaften der QCD-Materie diskutiert werden. In Kapitel IV wird die Rolle der Baryonenwechselwirkungen im Hadronenresonanzgas untersucht. Dies geschieht durch Anwendung des Beth-Uhlenbeck-Formalismus (BU) für repulsive HardcoreWechselwirkungen zwischen Baryonen. Der BU-Ansatz wird auch mit dem klassischen, excluded Volume modell (EV-Modell) "à la van der Waals" verglichen, bei dem Quanteneffekte vernachlässigt werden. Der zweite Virialkoeffizient $a_{2}$ - der excluded Volume Parameter, der im Rahmen des BU-Ansatzes berechnet wird, ist temperaturabhängig und unterscheidet sich drastisch vom klassischen EV-Modellergebnis. Bei Temperaturen von $T=100-200 \mathrm{MeV}$ unterschätzt das weit verbreitete klassische EV-Modell den EV-Parameter für Nukleonen bei einem bestimmten Wert des Nukleonen-Kernradius um Faktoren von 3-4. Die im Rahmen des BU-Ansatzes berechneten Werte von $a_{2}$ stimmen mit den Werten von $a_{2}$, die aus experimentell gemessenen Nukleonenphasenverschiebungen berechnet wurden, überein. Diese Ergebnisse weisen darauf hin, dass die vorherige Studien, in denen die Kernradien von Hadronen als Eingabe für das klassische EV-Modell verwendet wurden, unter Verwendung der entsprechend umskalierten EV-Parameter neu bewertet werden müssen.

Der zweite Teil dieser Arbeit besteht aus den Kapiteln V und VI. Hier wird ein einheitlicher Ansatz für die QCD-Materie das CMF-Modell vorgestellt. Das CMF- 
Modell umfasst viele Aspekte der QCD-Phänomenologie zusammen mit der zuvor diskutierten HRG-Beschreibung und den EV-Wechselwirkungen und ermöglicht eine einheitliche Beschreibung des Hadron-Quark-Übergangs, wodurch es für das gesamte QCD-Phasendiagramm anwendbar ist. Kapitel V der Arbeit beschreibt das CMF-Modell mit allen zugrunde liegenden Gleichungen und Freiheitsgraden im Detail. Die Parameter des Quarksektors des CMF-Modells wurden angepasst, um die Gitter-QCD-Daten zu beschreiben. Die Vorhersagen des CMF-Modells werden mit dem Schwerpunkt auf Fluktuationsmessungen diskutiert, von denen angenommen wird, dass sie die Phasenstruktur des Modells widerspiegeln. Die Werte der Netto-Baryonenladungskewness und der Kurtosis werden in der $T$ $\mu_{B}$-Ebene für isospin-symmetrische Materie dargestellt. Diese BaryonenzahlFluktuationsmessungen zeigen, dass das CMF-Modell die folgenden Übergänge hat: ein Phasenübergang erster Ordnung, Nuklearer Flüßig-Gas, Wiederherstellung der chiralen Symmetrie als Phasenübergang erster Ordnung, und ein glattes Crossover ist mit dem Deconfinement verbunden. Das CMF-Modell wird angewendet, um stabile Neutronensterne durch Lösen der Tolman-Oppenheimer-Volkoff-Gleichung zu modellieren. Die CMF-Vorhersagen für die Massen und Radien der NS stimmen mit den modernen astrophysikalischen Beobachtungen überein. Die berechneten Werte der NS-Gezeitenverformbarkeiten stimmen gut mit den Werten überein, die aus der Analyse von Gravitationswellen erhalten wurden, die von binären Neutronenstern-Verschmelzungen emittiert wurden.

Die verschiedenen Szenarien von Hardcore-Interaktionen im CMF-Modell werden in Kapitel VI untersucht. Die Werte der Volumenparameter von Hadronen werden durch die verfügbaren Gitter-QCD-Daten für die Suszeptibilitäten zweiter Ordnung $\chi_{i j}^{11}$ von Baryonen-, Elektro- und Seltsamkeitsladungen, $B, Q$ und $S$ eingeschränkt. Es wird festgestellt, dass $\chi_{i j}^{11}$ besonders empfindlich auf die kurzreichweitigen repulsive Wechselwirkungen von Hyperonen reagieren. Die Verringerung der Hyperonengröße im Vergleich zur Größe der nicht-seltsamen Baryonen verbessert die Übereinstimmung der CMF-Modellergebnisse mit den Gitter-QCD-Daten erheblich. Die von der elektrischen Ladung abhängigen Suszeptibilitäten sind empfindlich gegenüber dem kurzreichweitigen -Repulsivesvolumen der Mesonen. Der Vergleich mit Gitter-QCD-Daten deutet darauf hin, dass seltsame Baryonen, nicht-seltsame Mesonen und seltsame Mesonen deutlich kleinere Volumen haben als nicht-seltsame Baryonen. Das CMF-Modell mit diesen modifizierten Hadronenvolumen erlaubt eine hauptsächlich hadronische Beschreibung 
der QCD-Suszeptibilitäten wesentlich oberhalb der chiralen pseudokritischen Temperatur. Dieses verbesserte CMF-Modell, das auf den Gitter-QCD-Daten basiert, wurde verwendet, um die Eigenschaften sowohl der kalten QCD-Materie als auch der Neutronensternmaterie zu untersuchen. Die Phasenstruktur ist in beiden Fällen im Wesentlichen unverändert. Die Hyperonen überleben das Deconfinement zu höheren Dichten als nicht-seltsame Hadronen. Die maximale Masse des Neutronensterns bleibt etwa bei 2.1 $M_{\odot}$, und das Masse-Radius-Diagramm wird durch das Auftreten von Hyperonen nur geringfügig verändert und stimmt mit astrophysikalischen Beobachtungen überein. 


\section{Introduction}

On August 17th, 2017 the first gravitational wave (GW) signal from a binary neutron star (BNS) merger was recorded by LIGO and VIRGO detectors [10]. This event was the first long-sought gravitational wave signal coming from, at that time, only theorized neutron star collision. Such type of an event was long awaited since it allows to study dynamical properties of matter contained in neutron star interiors. The neutron stars are assumed to be the densest stable objects in the universe with central core densities reaching several times the density in nuclear cores $n_{0} \approx 10^{45}$ particles $/ \mathrm{m}^{3}$ and their sizes are of the order of $10 \mathrm{~km}$. An additional gravitational and the dynamical compression created by the merger creates an even more exotic environment where temperatures may reach up to $T \sim 100 \mathrm{MeV}$ which equals $\sim 10^{12}{ }^{\circ} \mathrm{C}$. At such extreme conditions, the matter properties are described by the theory of strong interactions - Quantum Chromodynamics (QCD). This detection was fascinating not only because it revealed the properties of macroscopic matter at extreme conditions, but also because the conditions were very similar to ones created in collisions of microscopic particles in the laboratory. It was pointed already in the early '50s [11, 12], even before the QCD was created, that similar extreme conditions can be created in a laboratory by colliding nuclei that have sizes of the order of $1 \mathrm{fm}=10^{-15} \mathrm{~m}$. Since then the idea of performing particle collision experiments to study extreme types of matter was dominant in high energy physics and allowed to gain a lot of knowledge on the extremely hot and dense matter before it was detected in neutron star mergers. 
In the second half of the 20th century the high energy particle collisions allowed to establish the most precise theory available today - the Standard Model of particle physics. The theory describes three out of four fundamental interactions, the electromagnetic, weak, and strong interactions, while the gravitational force still cannot be unified with the other three interactions. It can be split into two parts, the Quantum Chromodynamics, and the Quantum Electroweak theory. The Standard Model allows to classify all elementary particles (quarks, leptons, gluons, photons, Z- and W- bosons, and the Higgs boson), and to describe their interactions with exceptional accuracy. Only the quarks and gluons are subjects of strong interactions, while all of these elementary particles, except gluons, interact by the electroweak force. The quarks and leptons are fermions due to their half spin, they form the matter, while the other particles are bosons due to their integer spin, they mediate interactions among elementary particles. This classification allows to classify any composite particle which contains two or more elementary particles. High interest is attributed to hadrons, particles that are composed of quarks and virtual gluons, the properties of hadrons are described by means of QCD.

The Standard Model did help to answer many questions regarding the properties of composite and elementary particles, however, the complex structure of the theory does not allow to give precise predictions regarding possible macroscopic states of matter composed by these elementary and composite particles. Within this thesis, the main interest will be attributed to the different phases of strongly interacting matter, i.e., matter that is composed of particles that are subjected to strong interactions, the QCD matter. All features of the QCD theory are contained in its Lagrangian $\mathcal{L}_{\mathrm{QCD}}$ :

$$
\begin{aligned}
\mathcal{L}_{\mathrm{QCD}} & =\sum_{i, j} \bar{\psi}_{i}\left(i \gamma^{\mu}\left(\partial_{\mu} \delta_{i j}-\frac{i}{s} g \mathcal{A}_{\mu}^{a} \lambda_{a, i j}\right)-m_{i} \delta_{i j}\right) \psi_{j}-\frac{1}{4} G_{\mu \nu}^{a} G_{a}^{\mu \nu}, \\
G_{\mu \nu}^{a} & =\partial_{\mu} \mathcal{A}_{\nu}^{a}-\partial_{\nu} \mathcal{A}_{\mu}^{a}+g f^{a b c} \mathcal{A}_{\mu}^{b} \mathcal{A}_{\nu}^{c},
\end{aligned}
$$

here $\psi_{j}$ are quark fields, $\gamma_{\mu}$, are Dirac matrices, $g$ is the strong coupling constant, $\lambda_{a, i j}$ and $f^{a b c}$ are generators and structure constants of the $\mathrm{SU}(3)$ group respectively, $m_{i}$ is the mass of $i$-th quark. The summation over index $i$ goes over all known six quarks: up, down, strange, charm, bottom, and top. The $G_{\mu \nu}^{a}$ is the gauge invariant gluon field strength tensor and $A_{\mu}^{a}$ is the gluon field. Contrary to the electroweak theory where there are only two charges - electric and weak, which can be separated, i.e., each charge can be described within one theory, QCD has 
three conserved charges - three colors (ref, green, and blue) which can not be separated. Moreover, in QCD the gluons also carry a charge and can interact with each other by means of gluon exchanges. All in all, six different quark flavors, three different colors, gluons that can interact with each other, make QCD the most complicated fundamental theory. However, there is one separate feature of QCD that makes it outstanding, compared to the electroweak theory - quark confinement, a phenomenon that quarks and gluons can not be isolated and always form so-called color-neutral combinations, i.e. color-anticolor or in combinations of three different colors. Thus, quarks and gluons must always be confined in a group that forms a hadron. Asymptotic freedom is a feature of confinement that allows the coupling between quarks to become asymptotically weak at high energies or densities.

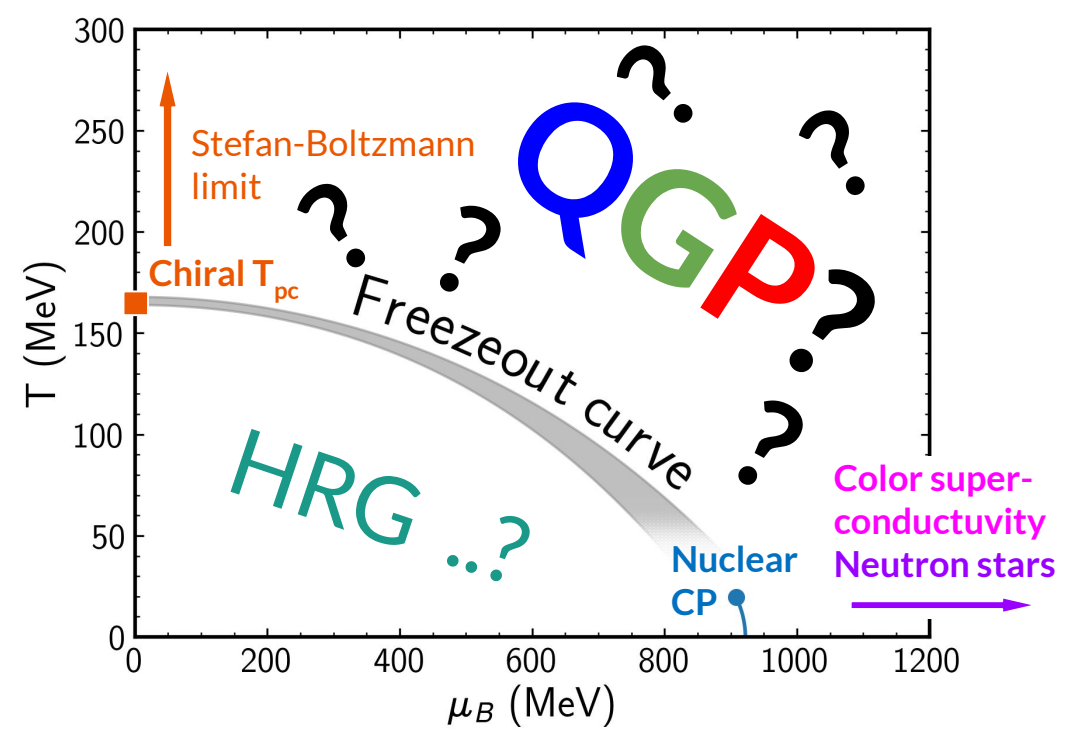

Figure I.1: Schematic view of the QCD phase diagram in temperature $T$ and baryon chemical potential $\mu_{B}$. At low energy densities the QCD matter is in a form of a hadron resonance gas, while at high energy densities the matter is present in a form of QGP. The mechanism behind the transition between the two phases is not known. The first principle Lattice QCD calculations allow to explore matter at vanishing baryon density, $\mu_{B}=0$. The heavy ion collisions probe the matter along the so-called freeze-out curve. The dense and cold QCD matter forms the cores of neutron stars.

The high number of QCD degrees of freedom hints that the QCD matter may 
have a rich phase diagram $[13,14,15,16,17]$. Although the QCD theory is well established, no direct calculations are possible and its phase structure remains unclear. The QCD theory suffers from calculation problems: the large coupling constant disfavors perturbative methods and the numerical sign problem prevents lattice (LQCD) calculations at finite densities. A big scientific interest is attracted to the state which is directly related to asymptotic freedom - the Quark-Gluon Plasma (QGP), where the energy densities are so high that quarks and gluons become free and form a new state of matter. A sketch of the QCD phase diagram with the current knowledge of its structure is presented in Fig. I.1.

Experimentally the QCD phase diagram is accessed via heavy ion collisions (HIC) where hundreds of neutrons and protons collide at high energy, so the kinetic energy is transformed into compression and heat to create a localized portion of hot QCD matter. The temperature and density of this QCD matter can be varied through the collision energy. However, there are numerous obstacles in relating heavy ion collisions to the QCD phase diagram due to the complicated dynamics that the system experiences due to the collision. After the violent shock transforms the colliding nuclei into a hot fireball which then cools and expands hence the temperature and density of created QCD matter varies. The heavy ion collisions are "recorded" by an experimental detector by measuring and identifying hadrons that yield from the fireball during its expansion. Surprisingly, the system with complicated dynamics and interactions can be described with just a few thermodynamic parameters which can be selected by analyzing the identified particle yields. This simplified description of heavy ion collisions allows mapping the latest stages of the collision to the QCD phase diagram via the so-called thermal fit procedure [18, 19, 20, 21]. Collision energy dependence of the extracted parameters, like temperature and chemical potential, defines the chemical freeze-out line $[22,23]$.

Currently, the state-of-the-art lattice QCD techniques offer the best approach to QCD matter by solving QCD at discretized space-time lattice. Due to the infamous sign problem, the LQCD methods are only available at vanishing baryon chemical potential $\mu_{B}=0$ which corresponds to a state where the number of baryons is equal to the number of anti-baryons. The LQCD simulations suggest that the transition from hadronic to QGP matter is a smooth crossover that takes place in a sizable region of temperatures $150 \mathrm{MeV} \lesssim T \lesssim 200 \mathrm{MeV}$, Fig. I.2. Due to the nature of LQCD methods, it is not possible to estimate quark and 


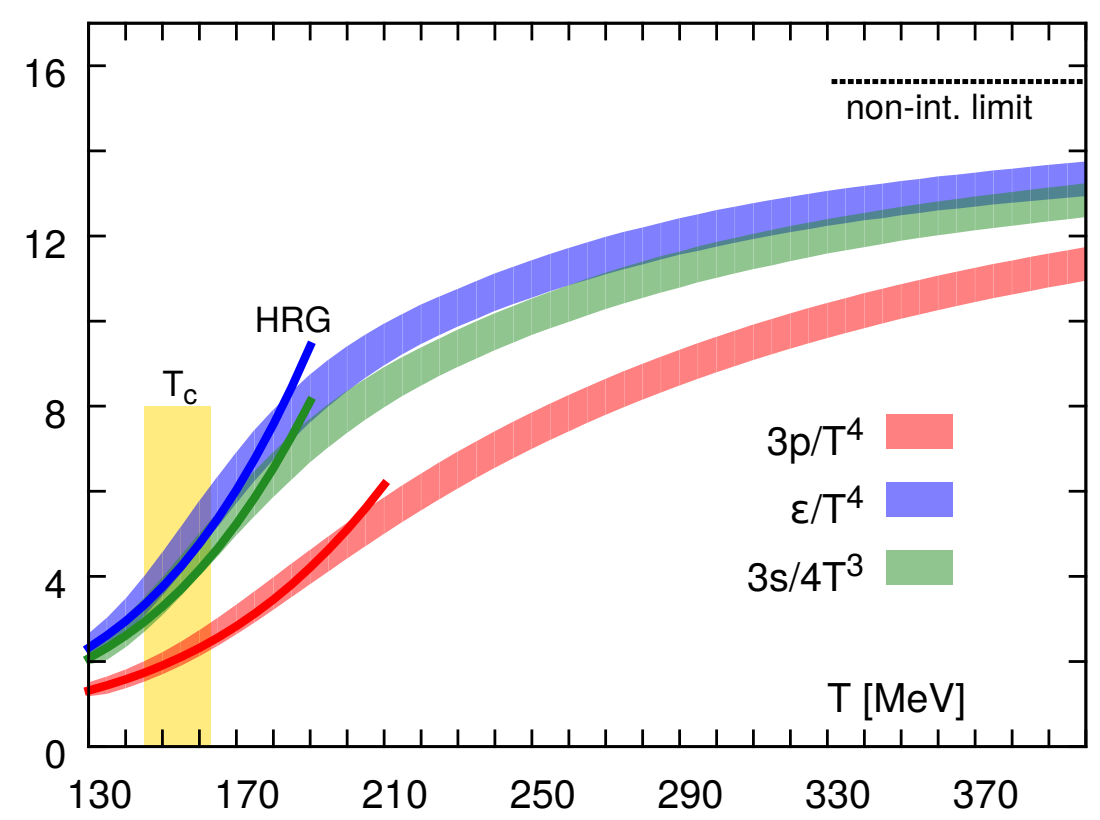

Figure I.2: The bands represent LQCD pressure, energy density, and entropy density as functions of the temperature at $\mu_{B}=0$. The dark lines correspond to the predictions of the HRG model. The horizontal line at $95 \pi^{2} / 60$ corresponds to the Stefan-Boltzmann limit for the energy density of massless 3 flavor quark-gluon gas. The yellow vertical band illustrates the location of the chiral pseudo-critical temperature, $T_{c}=(154 \pm 9) \mathrm{MeV}$. The figure is taken from [24].

hadron contributions separately at each temperature. An analysis of the socalled chiral condensate suggests a pseudocritical temperature of the transition $T_{\mathrm{pc}} \approx 154 \mathrm{MeV}[25,26]$. Significant interest is attributed to a possible critical point associated with the transition to QGP, it is conjectured that it is located at finite values of $\mu_{B}$ which are out of scope for the LQCD methods. The critical point scenario assumes that at some finite $\mu_{B}$ the smooth crossover to QGP state is replaced by a sharp first order transition, the point where this change occurs is called the critical point. Numerous phenomenological approaches to QCD favor this scenario [27, 17], however, these do not catch all aspects of QCD.

The LQCD results for the QCD equation of state at $\mu_{B}=0$, Fig. I.2, reveal several important phenomenological aspects of the QCD matter. For temperatures below $T \lesssim 170 \mathrm{MeV}$ the QCD thermodynamics can be described by the ideal Hadron Resonance Gas (HRG) model which represents an equilibrated gas of all known hadrons. This is the simplest approach for QCD matter which can be 
extended to finite values of chemical potentials. This is the HRG model which is widely used for the discussed before the description of the chemical freeze-out in heavy ion collisions. However, at some finite densities, the role of interactions between hadrons becomes prominent. For the higher temperatures $T \gtrsim 300 \mathrm{MeV}$ the thermodynamic quantities start to approach the Stefan-Boltzmann limit for non-interacting massless gas of quarks and gluons. However, the details of the transition between the two regimes remain unknown and phenomenological models are needed.

Another physical problem that can be used to explore another region of the QCD phase diagram is to study the properties of neutron stars (NS). The neutron stars are assumed to consist of neutron-dominated matter with densities several times of densities available inside of nuclei. With such large densities it is conjectured that a deconfined quark matter may be contained in NS interiors, however, no direct confirmation is present yet. The theoretical description of the NS relies on the knowledge of the QCD equation of state at zero temperature, $\mathrm{T}=0$, with an imposed condition of charge neutrality. Thus, astrophysical measurements of neutron star properties can be used to constrain the QCD equation of state at low temperatures. Recently, with the beginning of gravitational-wave astronomy, the detection and study of binary neutron star mergers became possible. This allows to analyze the properties of neutron stars at finite temperatures and to study new regions of the QCD phase diagram.

\section{I.1 Symmetries in fundamental physics}

Symmetries are the main mathematical tool in modern fundamental theoretical physics [28, 29]. All theories of fundamental interactions are based on symmetry relations: electroweak theory is based on the $S U(2) \times U(1)$ symmetry group, quantum chromodynamics is based on $S U(3)$ symmetry group, and general relativity is based on the assumption of local symmetry that the laws of nature should remain invariant under local changes of space-time coordinates, the famous equivalence principle. An interpretation of the Stern-Gerlach experiment and thus the discovery of particles' spin was possible only after the formulation of the exchange symmetry in quantum mechanics. The final discovery of particle physics and the last prediction of the Standard Model - the discovery of the Higgs boson is also an outcome of symmetries in theory. 


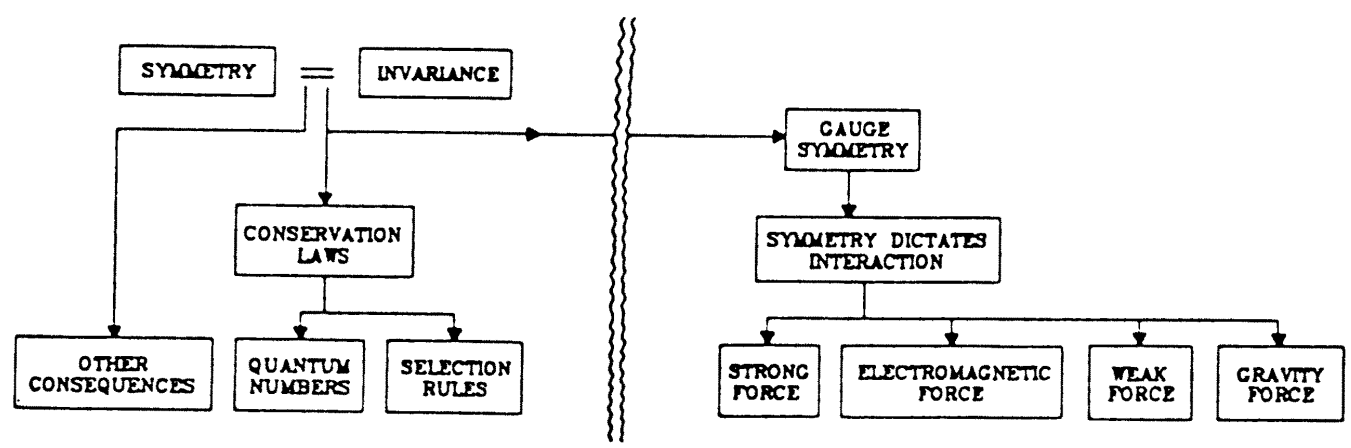

Figure I.3: The role of symmetry in fundamental Physics. Adapted from [29].

However, theory of symmetry didn't receive much attention in theoretical physics before the beginning of the 20th century. In 1905 Albert Einstein used symmetry arguments to formulate Maxwell's electrodynamics [30] which subsequently allowed to establish principles of special relativity. Then in 1918 Emmy Noether proved that every global continuous symmetry of a physical system has a corresponding conservation law [31]. The Noether theorem for some theory $\mathcal{L}(\psi(x))$ of field $\psi(x)$ with action $I(\psi)=\int d^{4} x \mathcal{L}(\psi(x))$ is formulated as follows:

$$
\psi(x) \rightarrow \psi(x)+\alpha \phi(x): 0=\delta I=\int d^{4} x \partial_{\mu}\left(\frac{\partial \mathcal{L}}{\partial\left(\partial_{\mu} \psi\right)} \phi(x)\right)
$$

where $\phi(x)$ is a generator of the symmetry. The invariance holds only on the condition of a conservation law:

$$
\partial_{\mu} j^{\mu}=0, \quad j^{\mu}=\frac{\partial \mathcal{L}}{\partial\left(\partial_{\mu} \psi\right)} \phi(x),
$$

this implies that the amount of a conserved quantity within a closed surface cannot change. A corresponding conserved charge $Q$ is defined as follows:

$$
Q=\int d^{3} x \mathrm{j}^{0}, \frac{d Q}{d t}=0
$$

The Noether theorem formalized a concept of conservation laws in theoretical physics: a conserved quantity is associated with every global continuous symmetry of the system. Thus, the energy conservation law is a result of time invariance, momentum is conserved as a result of translational invariance, and the angular momentum is conserved due to the rotation invariance.

Hand in hand with conservation laws the principle of gauge symmetry is used in fundamental physics. The concept of local gauge symmetry allows the 
formulation of physical forces in terms of fields that only can be measured up to some local gauge, which value does not affect the physical laws. This implies that the application of some local symmetry transformation doesn't change the description of the same physical situation. Historically the gauge symmetry was first discovered in the classical theory of electromagnetism. The electric field $\vec{E}$ and magnetic field $\vec{B}$ can be defined through a four-potential $A^{\mu}=(\phi, \vec{A})$ :

$$
\begin{array}{r}
\vec{E}=-\nabla \phi-\frac{\partial \vec{A}}{\partial t} \\
\vec{B}=\nabla \times \vec{A} .
\end{array}
$$

The four-potential $A^{\mu}$ can not be directly measured and can be inferred from measured values of $\vec{E}$ and $\vec{B}$ up to a constant in $A^{0} \rightarrow A^{0}+C$ and up to a gradient of some function in $\vec{A} \rightarrow \vec{A}+\nabla f$. The importance of the invariance of the Maxwell laws with respect to these gauge transformations was not fully understood until the significant development of quantum mechanics. In the quantum theory of electrodynamics, gauge symmetry is a feature of both electromagnetic waves, photons, and electron waves, electrons. These two symmetries are interrelated and are a result of $U(1)$ symmetry of the electron wave function. The QED Lagrangian reads as:

$$
\begin{array}{r}
\mathcal{L}_{\mathrm{QED}}=\bar{\psi}\left(i \gamma^{\mu} D_{\mu}-m\right) \psi-\frac{1}{4} F_{\mu \nu} F^{\mu \nu}, \\
D_{\mu}=\partial_{\mu}+i e A_{\mu}, \\
F_{\mu \nu}=\partial_{\mu} A_{\nu}-\partial_{\nu} A_{\mu},
\end{array}
$$

where $\psi$ is the electron quantum field, and $A_{\mu}$ is the photon quantum field. The electron field is required to obey $U(1)$ symmetry since only the phase difference of the wave function is measurable. The electron wave function $\psi$ should be invariant under the local $U(1)$ gauge transformation:

$$
\psi(x) \rightarrow \exp ^{i q \Theta(x)} \psi(x),
$$

this is only possible when the photon field $A_{\mu}$ transforms as:

$$
A_{\mu} \rightarrow A_{\mu}-i q \partial_{\mu} \Theta(x)
$$

In the language of the group theory, the electron field is in the fundamental representation and the photon field is in the adjoint representation of $U(1)$. This 
symmetry then, according to the Noether theorem, is associated with a conserved electric charge. Moreover, the $U(1)$ symmetry requires the photon to be massless. After the formulation of QED it was realized that in quantum field theories the interactions between conserved charges are associated with gauge symmetries.

Then in 1954 the idea of gauge of interactions was generalized mathematically by Yang and Mills [32] and extended to non-abelian symmetry groups such as $S U(N)$, however, at these times the theory was set aside. At these times particle physicists discovered an ever-growing number of hadrons. Such a plethora of particle species was assumed not to be fundamental. The particles were classified by charge, nuclear isospin, and strangeness by Gell-Mann and Nishijima [33, $34,35]$. This classification scheme led to the suggestion that the hadrons are composed of elementary particles - quarks which have three flavors, up, down, and strange. The discovery of $\Delta^{++}$and $\Omega^{-}$baryons became a benchmark for the hadron classification scheme. According to the Gall-Mann-Nishijima formula these hadrons should consist of three up and three strange quarks, respectively, which was forbidden by the Pauli principle, since quarks are fermions. This issue was suggested to resolve by an additional $S U(3)$ color charge attributed to quarks by Bogoliubov, Struminsky, Tavkhelidze [36], and separately by Greenberg [37], and by Han-Nambu [38]. With the concept of the color charge the nuclear force was naturally described as an $S U(3)$ gauge theory which was named Quantum Chromodynamics. QCD as any non-abelian theory, in contrast to QED which is abelian, has a different structure of the gauge field strength tensor $G_{\mu \nu}^{a}$ :

$$
G_{\mu \nu}^{a}=\partial_{\mu} \mathcal{A}_{\nu}^{a}-\partial_{\nu} \mathcal{A}_{\mu}^{a}+g f^{a b c} \mathcal{A}_{\mu}^{b} \mathcal{A}_{\nu}^{c}
$$

where the term $g f^{a b c} \mathcal{A}_{\mu}^{b} \mathcal{A}_{\nu}^{c}$ allows the QCD gauge bosons, gluons, to interact with each other and carry a color charge. In the QED theory, on the other hand, photons can not interact with each other directly. The self-interaction of the gauge bosons makes the QCD theory highly complicated since all equations become non-linear.

Then it was realized that the weak interaction which, for example, describes weak decay of the neutron, can be unified together with electromagnetism and described by $U(1) \times S U(2)$ gauge theory [40, 41, 42]. However, initially this theory predicted, besides then photon, three additional massless gauge bosons which were not observed yet. However, the short-range nature of the weak interactions suggested that these gauge bosons are massive. The problem was that no explicit 


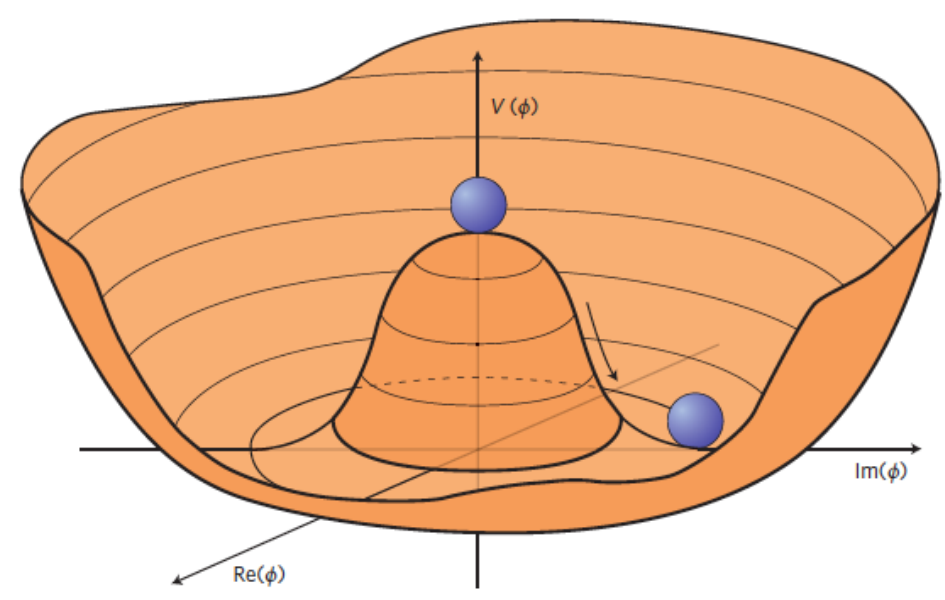

Figure I.4: Symmetry breaking effective 'Mexican hat' potential $V(\phi)$. The vacuum, the lowest energy state, is located at a random point around the lowest value of the potential. Adapted from [39].

mass term $\mathcal{L}_{\text {mass }}$ :

$$
\mathcal{L}_{\text {mass }}=-m^{2} A_{\mu}^{a} A_{a}^{\mu}
$$

is possible for a gauge field $A_{\mu}^{a}$ because it violates the $S U(N)$ symmetry under the gauge transformations:

$$
\begin{array}{r}
A_{\mu}^{a} \rightarrow A_{\mu}^{a}+\partial_{\mu} \alpha^{a}+g \epsilon_{a b c} A_{\mu}^{b} \alpha^{c}, \\
\mathcal{L}_{\text {mass }} \rightarrow-m^{2}\left|A_{\mu}^{a}+\partial_{\mu} \alpha^{a}+g \epsilon_{a b c} A_{\mu}^{b} \alpha^{c}\right|^{2} \neq \mathcal{L}_{\text {mass }}
\end{array}
$$

It was suggested by Englert and Brout, and separately Higgs [43, 44] that the mass of the weak bosons may be generated by a complex scalar field $\phi$ with a non-zero vacuum expectation value $\langle\psi\rangle=v$ given that the $\phi$ is also invariant under $S U(N)$ :

$$
\mathcal{L}_{s} \text { calar }=\left|D_{\mu} \phi\right|^{2}-\lambda\left(\phi^{\dagger} \phi-v^{2}\right)^{2}
$$

In this Lagrangian the coupling to the gauge field takes the form:

$$
-g^{2} \phi^{\dagger} \phi A^{\mu} A_{\nu}
$$

which resembles a mass term with dynamical mass $m^{2}=g^{2} \phi^{\dagger} \phi$ which is gauge invariant. The potential $V(\phi)=\left(\phi^{\dagger} \phi-v^{2}\right)^{2}$ ensures that at the vacuum expectation value is $\langle\psi\rangle=v$, so the mass of the gauge boson is $m^{2}=g^{2} v^{2}$. This is the 
Higgs mechanism of local gauge symmetry breaking which is a particular case of spontaneous symmetry breaking.

Spontaneous symmetry breaking is a process that appears in systems where the Lagrangian obeys symmetries but vacuum solutions do no exhibit the same symmetry. The origin of the symmetry breaking is clearly visible from the shape of the potential $V(\phi)$ as depicted in Fig. I.4. Even though the potential is symmetric, the lowest energy state is not and thus the system in a vacuum state favors the state with broken symmetry.

Spontaneous symmetry breaking is a useful tool for constructing effective field theories. It was used to describe quark interactions before the Higgs mechanism was proposed and QCD was formulated [46, 47]. In QCD an approximate chiral and flavor symmetry holds since the masses of up and down quarks can be approximately assumed negligible as compared to the QCD energy scale. Chiral symmetry assumes that the QCD Lagrangian is symmetrical under chiral transformations, transformations between the left and right quarks. This $S U(2)_{L} \times S U(2)_{R}$ symmetry implies that composite quark states, i.e. hadrons, are also chirally symmetrical which means that hadrons with the same quantum numbers but opposite parity

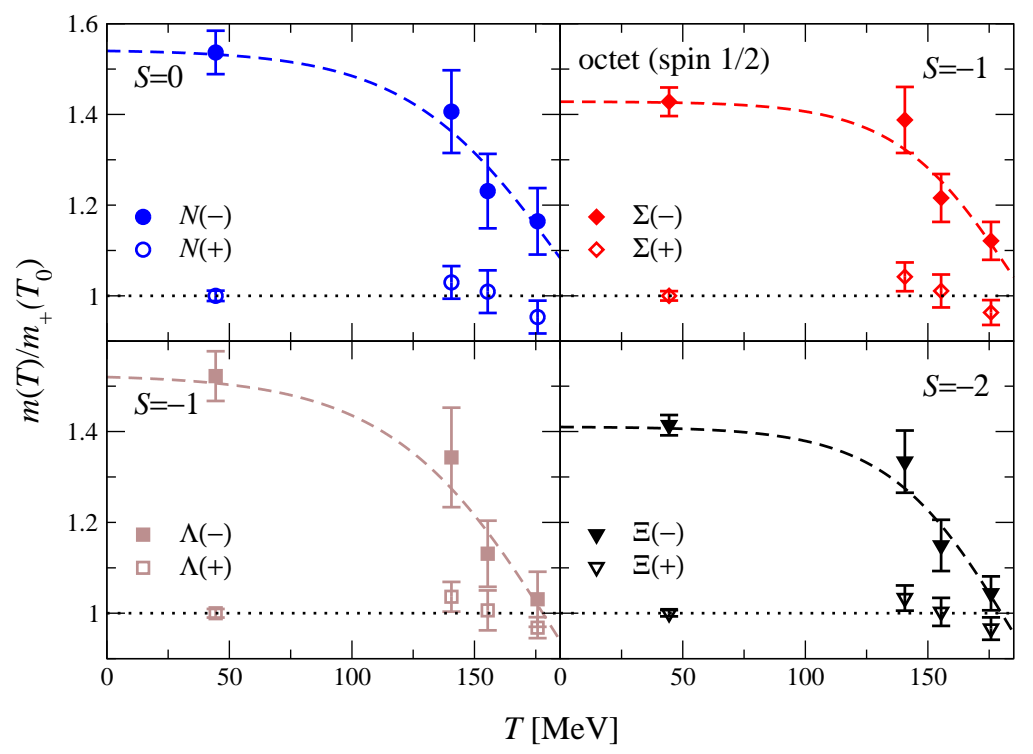

Figure I.5: Restoration of chiral symmtery in QCD at finite temperatures. The groundstate baryons become degenerate in mass with their parity partners as temperature rises. Adapted from [45]. 
have the same masses. However, no such symmetry is present in vacuum which means that chiral symmetry is spontaneously broken. The simplest Lagrangian which contains spontaneously broken chiral symmetry has the following form:

$$
\begin{aligned}
\mathcal{L}_{n_{f}=2}^{Q C D} \approx i \bar{\psi} \partial^{\mu} \gamma_{\mu} \psi+\frac{1}{2} \partial^{\mu} \vec{\pi} \partial_{\mu} \vec{\pi}+ & \frac{1}{2} \partial^{\mu} \sigma \partial_{\mu} \sigma- \\
& -g \bar{\psi}\left(\sigma+i \vec{\tau} \vec{\pi} \gamma_{5}\right) \psi-\lambda\left(\sigma^{2}+\vec{\pi}^{2}-v^{2}\right)^{2}
\end{aligned}
$$

here $\vec{\tau}$ are Pauli matrices, generators of $S U(2)$, and $\psi=(u, d)$. Since here not gauge, but global symmetry is broken, massless Goldstone bosons are not eaten by the gauge bosons and appear as interaction carriers. These are identified with pions and $\sigma$ meson.

Detailed first-principle lattice QCD calculations suggest that the chiral symmetry is indeed a feature of QCD. At high temperatures $T \approx m_{\pi}$ the masses of baryons become degenerate with the masses of baryons with the same quantum numbers but opposite parity [48, 45]. For example, nucleon becomes degenerate in mass with its parity partner $N^{*}$. Thus, at high energy densities the chiral symmetry becomes restored. 


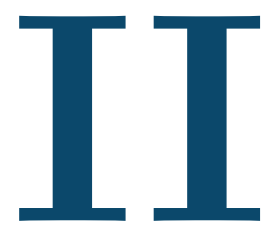

\section{Hadron yields and fluctuations in heavy ion collisions: thermal and transport model calculations}

The phase structure of the QCD matter is studied experimentally by measuring and analyzing hadron yields from laboratory heavy ion collisions. Only the latest, hadronic phase, of the fireball evolution created in the collisions reaches the detectors, thus, the full evolution of the system created in the collisions requires major effort to be reconstructed. However, the appearance of the transition to another phase is assumed to be reflected in the properties of several hadronic observables. The fluctuations of the measured hadron number, and special combinations of hadron yields, like the $K^{+} / \pi^{+}$ratio, are considered to be the most prominent. However, these observables are also sensitive to the dynamical effects of the collision, like collision geometry and finite acceptance of the experimental detector. In this chapter, the microscopic transport model simulations were performed for $\mathrm{p}+\mathrm{p}$, $\mathrm{Be}+\mathrm{Be}, \mathrm{Ar}+\mathrm{Sc}$, and $\mathrm{Pb}+\mathrm{Pb}$ collisions at the CERN Super Proton Synchrotron energy range to reveal the system size dependence of hadron production. The simulations results were compared with the thermal model predictions to evaluate a correct baseline for $\mathrm{K}^{+} / \pi^{+}$ratio and particle multiplicity fluctuations. The UrQMD transport model and Hadron Resonance Gas (HRG) models were used, 


\begin{tabular}{l|c|c|c|c|c|c} 
& \multicolumn{7}{|c}{$p_{\text {lab }}(A \mathrm{GeV} / c)$} \\
\hline NA49: $\mathrm{Pb}+\mathrm{Pb}$ & - & 20 & 30 & 40 & 80 & 158 \\
\hline NA61: $\mathrm{p}+\mathrm{p}$ & 13 & 20 & 31 & 40 & 80 & 158 \\
\hline NA61: Be+Be and Ar+Sc & 13 & 19 & 30 & 40 & 75 & 150
\end{tabular}

Table II.1: Collision momenta available at the SPS collider at CERN for the NA49, and NA61/SHINE collaborations.

so the considered scenarios were a priori without a transition to another phase of QCD. To mimic the experimental setup, the centrality selection and detector acceptance of the NA61/SHINE collaboration were reproduced in the simulations. A comparison with available experimental data was done. To understand a difference of the event-by-event fluctuations in $p+p$ and heavy ion collisions the centrality selection procedure in the sample of all inelastic $\mathrm{p}+\mathrm{p}$ events is proposed and analyzed within the UrQMD simulations.

This chapter is based on the research performed by the author of this thesis and published in Ref.[3].

\section{II.1 Canonical Ensemble Hadron Resonance Gas}

In the high multiplicity collisions of heavy nuclei, the formation of a statistical system is expected, this system is usually referenced as a fireball due to the estimated high temperatures in the interior. At the latest stages of the evolution, the system is in chemical and thermal equilibrium, so a hadron-resonance gas (HRG) description is applicable. The application of the HRG model to the hadron yields allows for a successful description of the chemical freeze-out in $\mathrm{p}+\mathrm{p}$ and $\mathrm{A}+\mathrm{A}$ collisions $[49,50,51,52]$, and references therein. In the case of $\mathrm{p}+\mathrm{p}$ collisions, the small size of the system requires exact charge conservation for each microstate of the statistical system. This leads to the Canonical Ensemble description. Here, the CE HRG calculations are performed for all systems in order to allow for a systematic comparison of the hadron production for both small and large systems within the HRG model. In large systems, i.e., $\mathrm{Pb}+\mathrm{Pb}$ collisions, the hadron yields at the SPS energies and higher, most of the CE results converge to the grand canonical ensemble (GCE) results. That is a result of the thermodynamic equivalence of the statistical ensembles. The GCE fixes the average values of the conserved charges, but their changes from one microstate to another are allowed. 
The e-by-e hadron number fluctuations are naturally different in the CE and GCE in the thermodynamic limit [53].

Here, a non-interacting CE HRG description is used in accord with previous works $[50,52]$. The calculations are performed using a publicly available Thermal-FIST package [54]. The CE description utilizes the following free parameters:

$$
T, V, \gamma_{S},
$$

where $T$ and $V$ are, respectively, the system temperature and volume at the chemical freeze-out, and $\gamma_{S}[55,56,57]$ is the strangeness suppression parameter that implements the incomplete chemical equilibration of strange hadrons. In the following the system radius $R \equiv[3 V /(4 \pi)]^{1 / 3}$ is used instead of volume $V$ for convenience. Additional constraints on the baryon number $B$, electric charge $Q$, and net strangeness $S=0$ are imposed to satisfy the conservation laws given by the content of the colliding nuclei.

As a first step to describe the system size dependence in a transparent way, the complete strangeness equilibrium, $\gamma_{S}=1$, is assumed and the same value of temperature $T$ for all colliding systems is considered. The values of $T$ at different collision energies are taken in the form of the freeze-out curve parameterization from [22]:

$$
T\left(\mu_{B}\right)=a-b \mu_{B}^{2}-c \mu_{B}^{4}, \quad \mu_{B}\left(\sqrt{s_{N N}}\right)=\frac{d}{1+e \sqrt{s_{N N}}}
$$

where the parameters $a, b, c, d$, and $e$ are extracted from fits to the central $\mathrm{Pb}+\mathrm{Pb}$ data at the SPS energies as in Ref. [50]. The $\sqrt{s_{N N}}$ is the center of mass energy of the nucleon pair in the colliding systems. With the above assumptions, the considered systems only differ at the freeze-out by the size $R$.

The system size at the freeze-out can be calculated by using an approximation of equal baryon densities $\rho_{B}$ in all considered $\mathrm{A}+\mathrm{A}$ systems, and assuming that this value of $\rho_{B}$ is equal to the value found in the GCE HRG with Eq. (II.2). The function $\rho_{B}\left(\sqrt{s_{N N}}\right)$ calculated in this way is shown in Fig. II.1 (a). The radius then can be calculated as $R=\left[3 B /\left(4 \pi \rho_{B}\right)\right]^{1 / 3}$, where total baryonic number $B$ equals the number of nucleon participants. The radius of different systems studied by the NA61/SHINE Collaboration is shown in Fig. II.1 (b). These results may be modified by the presence of nonzero baryon proper volumes which would lead to smaller $\rho_{B}$ and larger $R$ values [58]. 

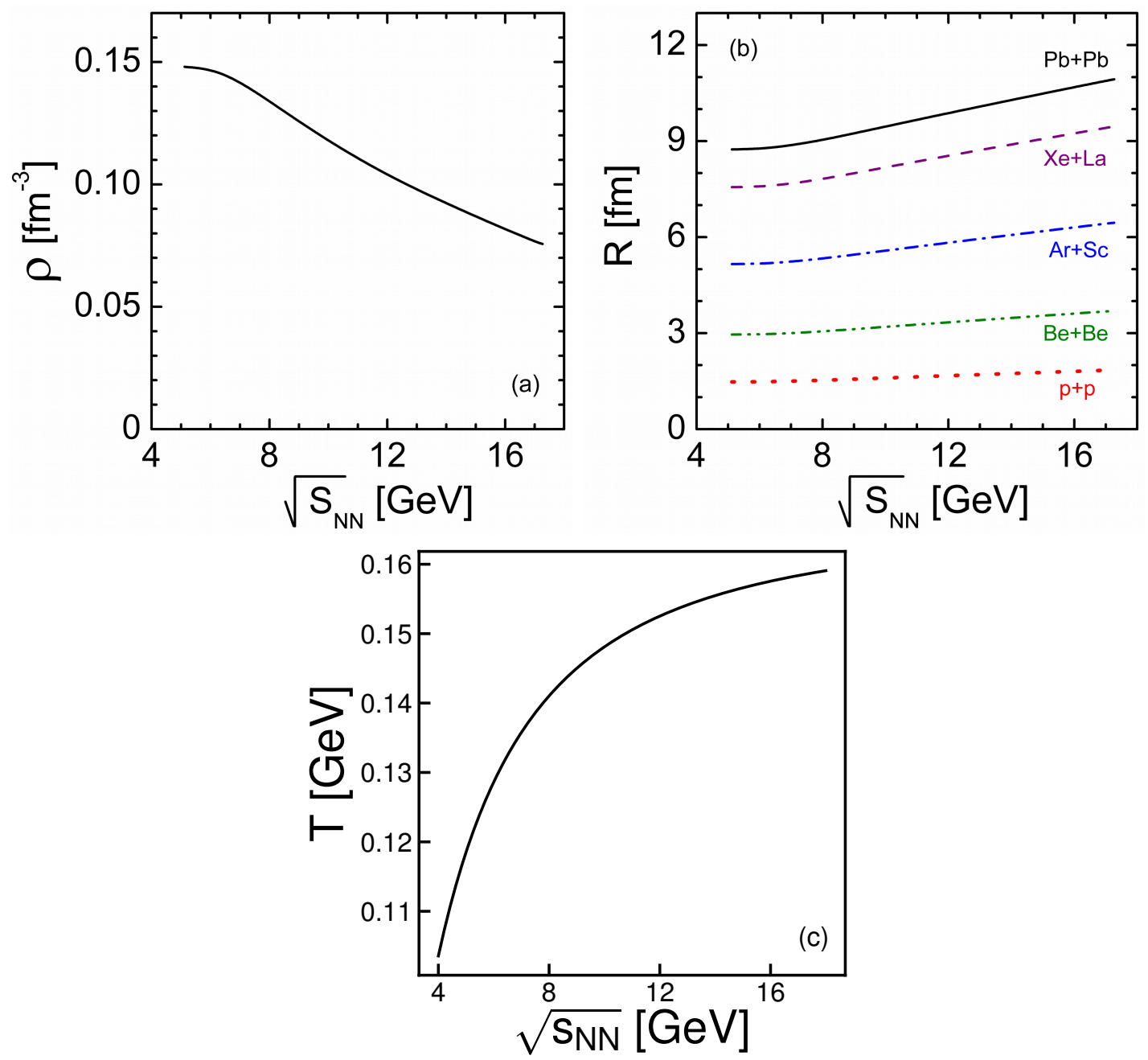

Figure II.1: The baryon density $\rho_{B}(\mathrm{a})$, the radius $R$ (b), and the temperature $T$ (c) calculated with CE HRG of the fireball at the chemical freeze-out as functions of the collision energy.

In the GCE, the intensive quantities $K^{+} / \pi^{+}$and $\omega^{-}$are the functions of $T$ and $\mu_{B}$, and are not sensitive to the system volume $V$. In the CE the global charge conservation introduces sensitivity to the volume and suppresses the mean multiplicities of hadrons in small systems. Significant CE suppression effects happen for hadron yields when the total number of particles and antiparticles of that corresponding conserved charge is of an order of unity or smaller. This leads, e.g., to $\left\langle K^{+}\right\rangle_{\mathrm{CE}}<\left\langle K^{+}\right\rangle_{\mathrm{GCE}}$ at finite values of $V$ because of the exact strangeness conservation. This difference of the hadron yields in the CE and GCE becomes 
negligible in the thermodynamic limit $V \rightarrow \infty$. On the other hand, the smaller $\mathrm{CE}$ values of the particle number fluctuations, e.g., $\omega_{\mathrm{CE}}^{-}<\omega_{\mathrm{GCE}}^{-} \cong 1$, are just most pronounced in the thermodynamic limit.
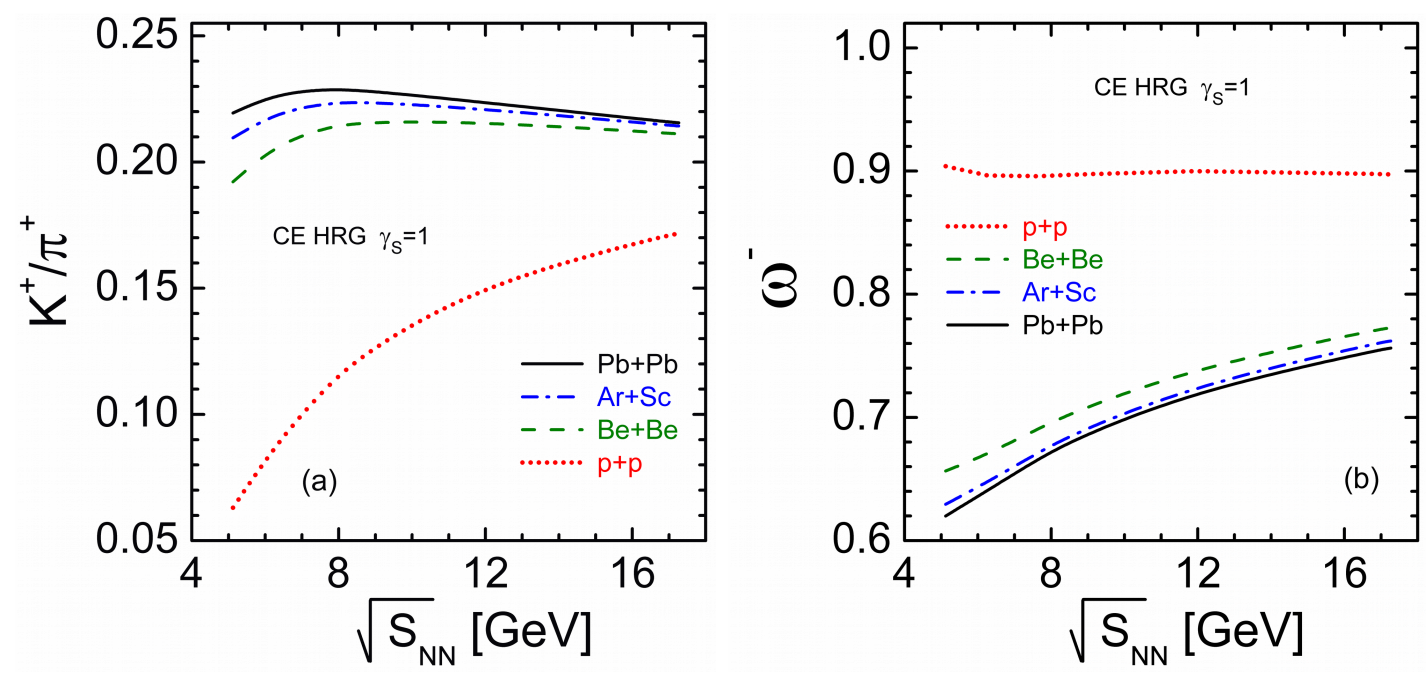

Figure II.2: The $K^{+} / \pi^{+}$ratio and the scaled variance $\omega^{-}$of negatively charged particles calculated with CE HRG along at the fireball freeze-out as functions of the collision energy.

The CE effects due to the charge conservation can be observed in Fig. II.2, i.e., $\left(K^{+} / \pi^{+}\right)_{\mathrm{A}+\mathrm{A}}>\left(K^{+} / \pi^{+}\right)_{\mathrm{p}+\mathrm{p}}$ and $\left(\omega^{-}\right)_{\mathrm{A}+\mathrm{A}}<\left(\omega^{-}\right)_{\mathrm{p}+\mathrm{p}}$. The $K^{+} / \pi^{+}$ratio increases and $\omega^{-}$decreases monotonously with the size of colliding systems. Therefore, the CE suppression is stronger for the particle yields in small systems like $p+p$, and for particle number fluctuations in large systems like Pb+Pb, Figs. II.2 (a) and (b), respectively (see also Ref. [53]). The differences between $\mathrm{p}+\mathrm{p}$ and $\mathrm{A}+\mathrm{A}$ collisions are stronger at the smallest collision energies. These features of the $\mathrm{CE}$ HRG are similar to those observed in the data.

\section{II.1.1 Strangeness suppression and acceptance corrections in HRG}

A comprehensive comparison between the CE HRG results and the data requires additional concepts to be incorporated in the model, namely, incomplete equilibration of strange hadrons and finite acceptance of detectors.

Previous analysis of the experimental hadron yields demonstrated suppression of strange hadrons as compared to the predictions of the HRG model, which is interpreted as an incomplete strangeness equilibration. That is usually treated 
by the strangeness suppression factor $\gamma_{S}[59,55,56,57]$ with numerical values in the range of $0.5<\gamma_{S}<1$ for the SPS energies. The suppression factor $\gamma_{S}^{n}$ takes into account the quark content of each hadron by considering the number $n$ of strange quarks and/or antiquarks in the given hadron. The small values of $\gamma_{S} \sim 0.5$ correspond to $\mathrm{p}+\mathrm{p}$ at small SPS energies, larger $\gamma_{S} \sim 1$ to central collisions of heavy nuclei, and large SPS energies [60].

By default, the HRG model considers contributions to the hadron multiplicities from the whole phase space, i.e., the so-called full $4 \pi$ acceptance. In the lab frame experiments, as NA61 is, the averaged full $4 \pi$ particle yields can be measured by mirroring the yields measured at positive rapidity to the negative rapidity region. However, that is not applicable for fluctuation studies, and only the fluctuations of particles at the forward rapidity region are measurable. The acceptance can be considered by the assumption that the probability of a particle to be detected governs binomial statistics. This assumption leads to a so-called binomial acceptance correction to the HRG model, here the scaled variance with respect to the experimental acceptance can be calculated by simple formula (see, e.g., [53])

$$
\omega^{-}=1-q+q \omega_{4 \pi}^{-} .
$$

In Eq. (II.3), $q=\left\langle N_{-}\right\rangle /\left\langle N_{-}\right\rangle_{4 \pi}$, where $\left\langle N_{-}\right\rangle$and $\left\langle N_{-}\right\rangle_{4 \pi}$ are the average $N_{-}$ values in the accepted region and in the full phase space, respectively, $\omega^{-}$and $\left(\omega^{-}\right)_{4 \pi}$ denote the scaled variances of the accepted hadrons and all final hadrons, respectively. The binomial acceptance is a reasonable approximation in most cases, but can be violated in some scenarios (see, e.g., Ref. [61]).

\section{II.2 Hadron yields in the UrQMD model}

The transport model simulations of heavy ion collisions allow to study the dynamics of the collisions in great detail, here the UrQMD model $[62,63]$ will be used as a well-adopted approach for medium energy heavy ion collisions. The UrQMD model operates on the hadronic level and directly allows to estimate effects of finite acceptance, centrality selection, and strangeness suppression. 


\section{II.2.1 Centrality selection in $A+A$ collisions}

Before studying the hadron yields in heavy ion collisions, the proper centrality selection should be implemented. For this purpose, the NA61/SHINE Collaboration uses the Projectile Spectator Detector (PSD) to measure the forward energy $E_{\mathrm{F}}$, these values then can be used to quantify the centrality of an event. This is the energy deposited in a small angle of the forward hemisphere where the spectators are assumed to be located. The calorimeter is not able to identify particles, thus, not only the projectile spectators contribute to $E_{\mathrm{F}}$. For a proper comparison with the experimental data, the experimental centrality selection procedures should be replicated and the detector acceptance should be imposed in the UrQMD simulations. This is done in $\mathrm{Be}+\mathrm{Be}$ and $\mathrm{Ar}+\mathrm{Sc}$ collisions with the acceptance maps of the PSD and NA61/SHINE detectors [64]. For the Pb+Pb collisions the zero value of the impact parameter $b$ can be used as a good approximation to $1 \%$ most central collisions (see, e.g., Refs. [65, 66]), and so only the acceptance maps of the NA61/SHINE detectors are to be imposed.

The selection of an appropriate centrality class is a special task. One has to select an appropriate sample of events with a sufficient number of events that correspond to some centrality to minimize the 'background fluctuations'. By studying events from one narrow centrality class, only the fluctuations that originate from strong interactions are expected to contribute to the particle production, so geometrical fluctuations of the impact parameter $b$ which change the number of participating nucleons are removed from the event sample. The centrality classes obtained in the UrQMD simulations with the PSD acceptance maps are presented in Fig. II.3. Ideally, these distributions should have strong peaks located at $E_{\mathrm{F}}=k \cdot E_{\text {lab }}$ where $E_{\text {lab }}$ is the projectile energy and $k$ is an integer number, so each peak will correspond to $k$ spectators in the event. However, the distributions are rather smooth since contributions from every single participant are smeared out by secondary particles that fly into the forward calorimeter. However, for small systems and/or low energies, the peaks still can be identified to quantify the contribution from a single participant. For example, in Be+Be at $30 A \mathrm{GeV}$ one can observe 7 peaks that are produced by 1-7 spectator nucleons in the Be+Be collision, see Fig. II.3(a).

The effect of centrality selection on the scaled variance $\omega^{-}$is shown in Fig. II.4. The values of scaled variance $\omega^{-}$saturate for $1 \%$ most central collision events and increase in the wider samples of collision events. This is, as discussed before, an 
effect due to the large fluctuations of the number of nucleon participants which has mostly geometrical origin.
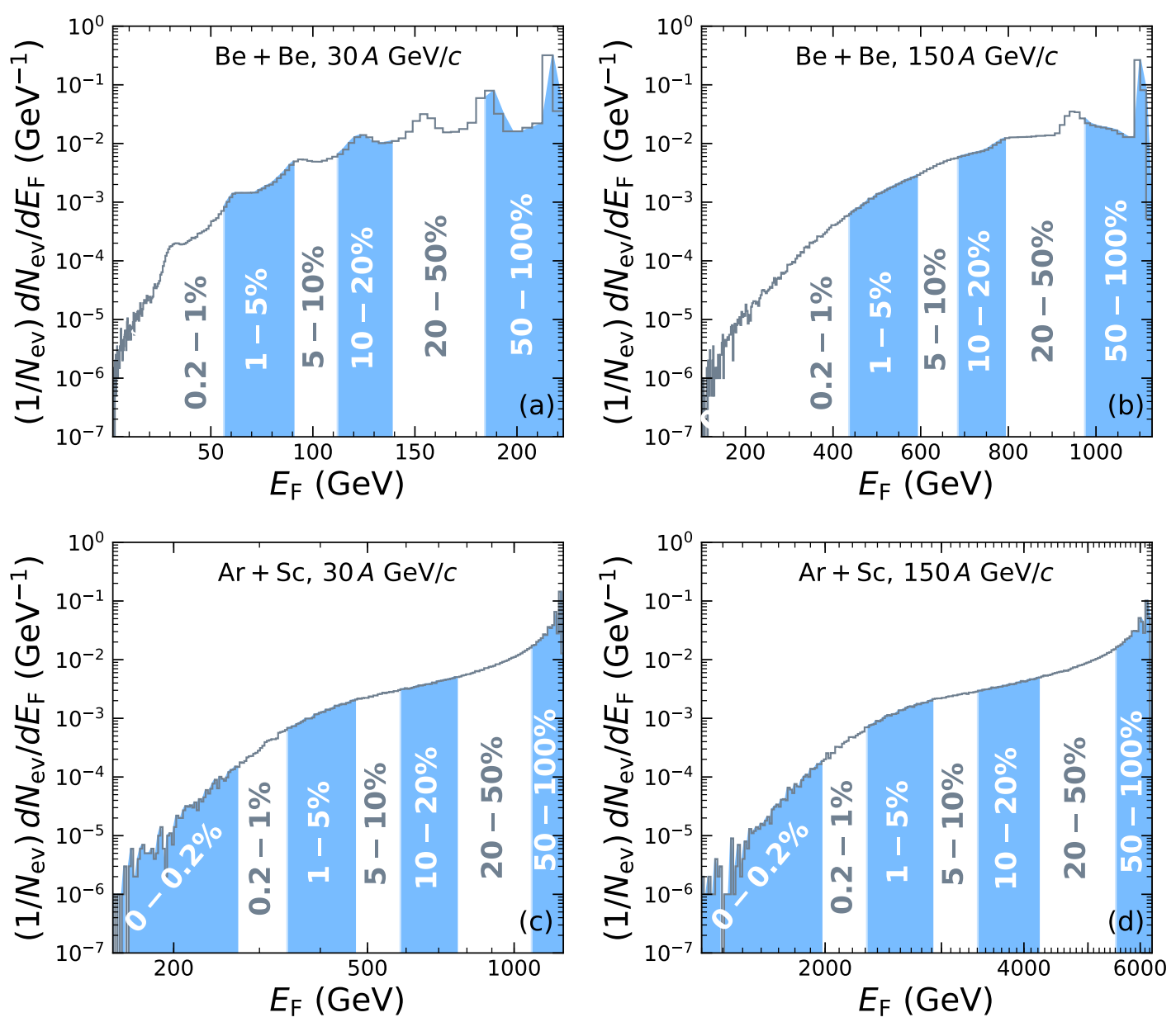

Figure II.3: Numbers of events as a functions of the forward energy $E_{\mathrm{F}}$ calculated in the minimum bias UrQMD simulations for $\mathrm{Ar}+\mathrm{Sc}$ and $\mathrm{Be}+\mathrm{Be}$ collisions at $E_{\text {lab }}=30$ and $150 \mathrm{AGeV} / \mathrm{c}$. The colored bands correspond to different centrality classes that are indicated with respective numbers.

The sufficient centrality class to study the particle number fluctuations can be also selected using a comparison of $\omega^{-}$with the strongly intensive analog of the scaled variance $-\Omega\left[N_{-}, E_{\mathrm{P}}\right][67]$ :

$$
\Omega\left[N_{-}, E_{\mathrm{P}}\right]=\omega^{-}-\left(\left\langle N_{-} E_{\mathrm{P}}\right\rangle-\left\langle N_{-}\right\rangle \cdot\left\langle E_{\mathrm{P}}\right\rangle\right) /\left\langle E_{\mathrm{P}}\right\rangle, \quad E_{\mathrm{P}}=E_{\text {beam }}-E_{\mathrm{F}} .
$$

The calculations confirm that $\Omega\left[N_{-}, E_{\mathrm{P}}\right]$ is almost insensitive to the centrality 

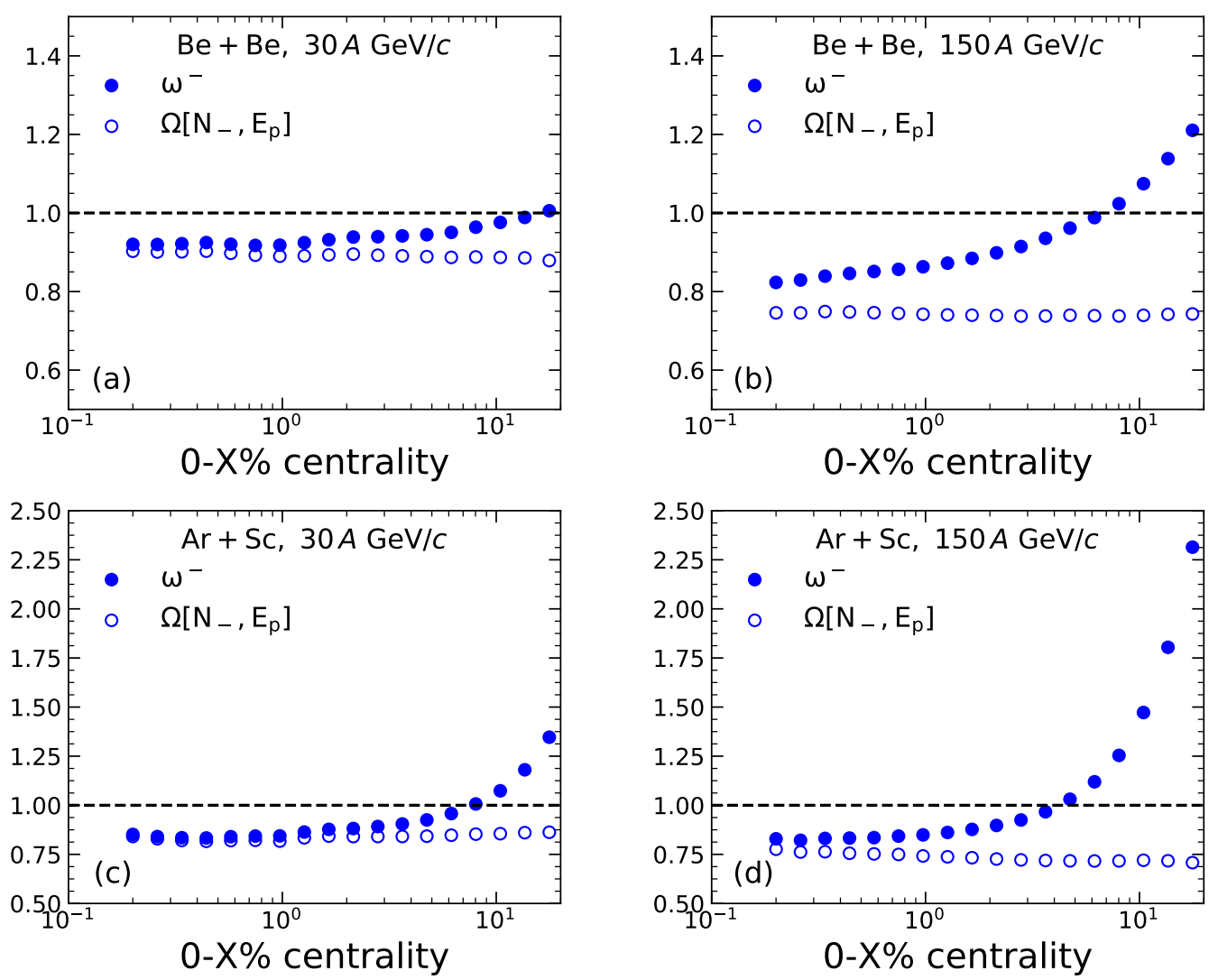

Figure II.4: The UrQMD results for the scaled variance $\omega^{-}$(full symbols) and strongly intensive quantity $\Omega\left[N_{-}, E_{\mathrm{P}}\right]$ (open symbols) as a functions of the centrality for $\mathrm{Be}+\mathrm{Be}$ (top) and $\mathrm{Ar}+\mathrm{Sc}$ (bottom) collisions at $p_{\text {lab }}=30 \mathrm{~A} \mathrm{GeV} / \mathrm{c}$ (left) and $p_{\text {lab }}=150 \mathrm{~A} \mathrm{GeV} / \mathrm{c}$ (right).

class, see Fig. II.4. Moreover, the saturation of $\omega^{-}$does occur in the region of centralities where $\omega^{-} \cong \Omega\left[N_{-}, E_{\mathrm{P}}\right]$.

\section{II.2.2 Centrality selection in $p+p$ collisions}

Contrary to $A+A$ ion collisions, the proton-proton collisions are usually divided only into two classes: elastic and inelastic, since the number of participants is always fixed to two, and the geometric fluctuations are hard to interpret. However, the centrality selection based on forward energy $E_{\mathrm{F}}$ can be introduced also to $p+p$ which may give some intuition about the geometric fluctuations there. Moreover, it is important in the context of recent observation by the NA61 collaboration where it was found that multiplicity fluctuations in $p+p$ are smaller than in most 
central $A+A$ collisions. In the model of independent sources, particularly in the wounded nucleon model [68], where A+A collisions are considered as independent nucleon-nucleon collisions, the scaled variance $\omega^{-}$can be presented as [68]

$$
\omega_{\mathrm{A}+\mathrm{A}}^{-}=\omega_{\mathrm{N}+\mathrm{N}}^{-}+\frac{1}{2}\left\langle n_{-}\right\rangle_{\mathrm{N}+\mathrm{N}} \omega_{\text {part }} .
$$

Here $\omega_{\mathrm{N}+\mathrm{N}}^{-}$and $\left\langle n_{-}\right\rangle_{\mathrm{N}+\mathrm{N}}$ are, respectively, the scaled variance and mean multiplicities of negatively charged hadrons in nucleon-nucleon collisions, and $\omega_{\text {part }}$ is the scaled variance for the e-by-e fluctuations of the nucleon participants. Under these assumptions, $\omega^{-}$in any $\mathrm{A}+\mathrm{A}$ collisions is larger than in $\mathrm{p}+\mathrm{p}$ collisions, $\omega_{\mathrm{A}+\mathrm{A}}^{-} \geq \omega_{\mathrm{p}+\mathrm{p}}^{-}$. To have $\omega_{\mathrm{A}+\mathrm{A}}^{-} \cong \omega_{\mathrm{p}+\mathrm{p}}^{-}$one needs $\omega_{\text {part }} \cong 0$ which may be possible for a very rigid centrality selection in A+A collisions. However, Eq. (II.5) is evidently in contradiction with $\omega_{\mathrm{p}+\mathrm{p}}^{-}>\omega_{\mathrm{Pb}+\mathrm{Pb}}^{-}$seen in the data of NA61 collaboration. The experimental data suggests that at the largest SPS collision energies the $\omega_{\mathrm{p}+\mathrm{p}}^{-}>1$, and $\omega_{\mathrm{A}+\mathrm{A}}^{-}<1$ for most central heavy ion collisions.

What is the origin of $\omega_{\mathrm{p}+\mathrm{p}}^{-}>1$ at large collision energies? One could argue that the main reason for the large e-by-e fluctuations of hadron multiplicities is the absence of the 'centrality selection' in $\mathrm{p}+\mathrm{p}$ inelastic reactions.

This problem can be tackled by an introduction of the 'centrality selection' for $\mathrm{p}+\mathrm{p}$ inelastic reactions within the UrQMD simulations. The centrality samples in $\mathrm{p}+\mathrm{p}$ inelastic reactions may be defined by the values of $E_{\mathrm{F}}$ the same as in the $\mathrm{A}+\mathrm{A}$ collisions. Here a physical interpretation of these different centralities in $\mathrm{p}+\mathrm{p}$ reactions is omitted. The $\mathrm{Be}+\mathrm{Be}$ experimental maps to calculate the energy deposited in the PSD are used due to the lack of the PSD acceptance maps for $\mathrm{p}+\mathrm{p}$ collisions. The UrQMD results for the 'centrality samples' in $\mathrm{p}+\mathrm{p}$ inelastic reactions are shown in Figs. II.5 (a) and (b). For the $\mathrm{p}+\mathrm{p}$ inelastic reactions, there is always a non-vanishing probability that no particle is emitted to the forward energy calorimeter due to the relatively low particle multiplicity. Among inelastic $\mathrm{p}+\mathrm{p}$ collisions at $p_{\text {lab }}=31 \mathrm{GeV} / c$ and $158 \mathrm{GeV} / c$ there are, respectively, about $48 \%$ and $13 \%$ of inelastic collision events with $E_{\mathrm{F}}=0$. The samples with $E_{\mathrm{F}}=0$ are thus considered as the most central $\mathrm{p}+\mathrm{p}$ inelastic reactions. In future experimental improvements, a more precise centrality triggering may be possible.

The change in scaled variance $\omega^{-}$in $p+p$ collisions due to the introduced centrality selection and a comparison with strongly intensive $\Omega\left[N_{-}, E_{\mathrm{P}}\right]$ is shown in Figs. II.5 (c) and (d). At $p_{\text {lab }}=158 \mathrm{~A} \mathrm{GeV} / c$ the value of $\omega^{-} \cong 1.15$ is obtained for all inelastic $\mathrm{p}+\mathrm{p}$ reactions and $\omega^{-} \cong 0.76$ for $13 \%$ most central $\mathrm{p}+\mathrm{p}$ collisions 

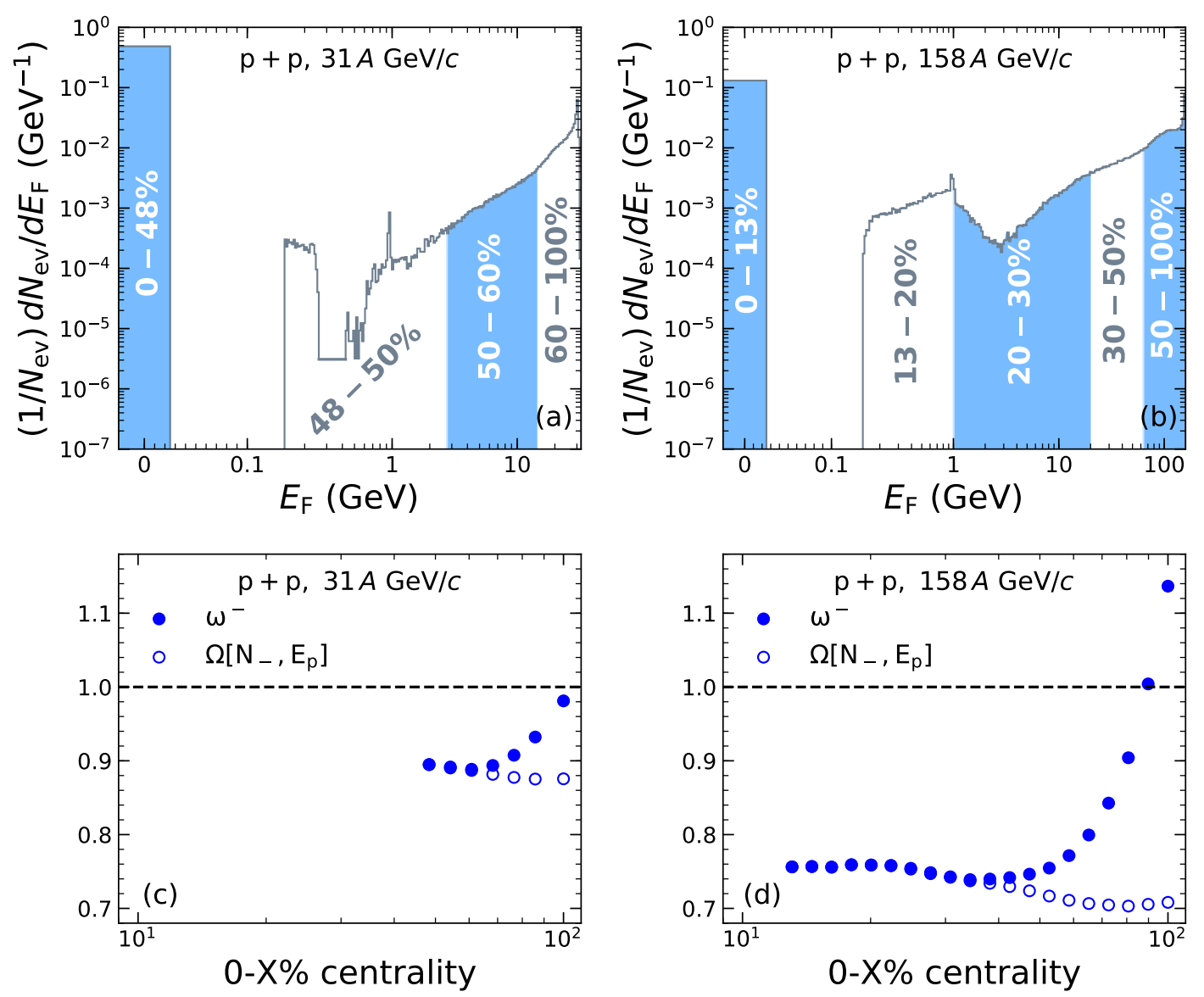

Figure II.5: The numbers of events as functions of the forward energy $E_{\mathrm{F}}$ calculated within UrQMD for inelastic $\mathrm{p}+\mathrm{p}$ collisions at $p_{\text {lab }}=30 \mathrm{~A} \mathrm{GeV} / \mathrm{c}$ (a) and $p_{\text {lab }}=150 \mathrm{~A} \mathrm{GeV} / \mathrm{c}(\mathrm{b})$. The scaled variance $\omega^{-}$(full symbols) and strongly intensive scaled variance $\Omega\left[N_{-}, E_{\mathrm{P}}\right]$ (open symbols) are presented as functions of the centrality for inelastic $\mathrm{p}+\mathrm{p}$ collisions at $p_{\mathrm{lab}}=30 \mathrm{~A} \mathrm{GeV} / \mathrm{c}(\mathrm{c})$ and $p_{\mathrm{lab}}=150 \mathrm{~A} \mathrm{GeV} / \mathrm{c}$ (d).

which illustrates a significant lowering of fluctuations in the most central samples.

\section{II.3 Comparison with the experimental data}

Here comparisons of the model calculations with the experimental data of NA61/SHINE Collaboration and older $\mathrm{Pb}+\mathrm{Pb}$ data of the NA49 Collaboration are presented.

The CE HRG results are depicted in Figs. II.6 there $\gamma_{S}=1$ was fixed. The values of $\gamma_{S}$ almost have no effect on the $\omega^{-}$, since the majority of negative charge 
is carried by non-strange $\pi^{-}$mesons. A monotonous decrease of the $4 \pi$ values of $\omega^{-}$ with the atomic number of colliding nuclei is predicted by the CE HRG which was presented in Fig. II.2 (b). The results presented in Figs. II.6 (c) and (d) are based on the binomial acceptance model in Eq. (II.3) where the acceptance parameter $q$ was estimated from microscopical simulations of the detector acceptance. The non-monotonous behavior of $\omega^{-}$with $A$ seen in Figs. II.6 (c) and (d) for the accepted particles appear only because of the smaller values of the acceptance parameter $q$ in Eq. (II.3) for $\mathrm{Pb}+\mathrm{Pb}$ collisions $(q \cong 0.06$ and $q \cong 0.16$ for 30 and $158 A \mathrm{GeV} / c$, respectively) in comparison to other $\mathrm{A}+\mathrm{A}$ and $\mathrm{p}+\mathrm{p}$ reactions ( $q \cong 0.3$ and $q \cong 0.4$ for $30 / 31$ and $150 / 158 A \mathrm{GeV} / c$, respectively).

The value of $\omega^{-}<1$ are observed for all colliding systems at $30 / 31 \mathrm{AGeV} / c$ and for large systems, namely $\mathrm{Ar}+\mathrm{Sc}$ and $\mathrm{Pb}+\mathrm{Pb}$, at the highest available SPS energy, 150/158 A GeV/c. All these data can be qualitatively described by the CE HRG. However, for the two smallest systems, $\mathrm{p}+\mathrm{p}$ and $\mathrm{Be}+\mathrm{Be}$, at 150/158 A GeV/c the data correspond to the large values, $\omega^{-}>1$. In these two cases, the CE HRG results are in contradiction with the data.

As seen from Figs. II.7 (a) and (b) the UrQMD values of the $K^{+} / \pi^{+}$ratio are systematically smaller than the experimental ones. In the UrQMD model the strange hadron production is underestimated because only the two-body hadron collisions are possible and no partonic degrees of freedom are excited [72]. In the transport approach which includes the parton degrees of freedom, the strange hadron production seems to be in agreement with the data [73]. On the other hand, a monotonic increase of the $K^{+} / \pi^{+}$ratio with the size of colliding nuclei observed in the data is reproduced by the UrQMD simulations.

The scaled variance $\omega^{-}$is very sensitive to the centrality selection. Figures II.7 (c) and (d) present a comparison of the UrQMD results with the available data for $\omega^{-}$. The UrQMD model produces a satisfactory agreement with the $\omega^{-}$ data for $\mathrm{Ar}+\mathrm{Sc}$ and $\mathrm{Pb}+\mathrm{Pb}$ central collisions, and for all inelastic $\mathrm{p}+\mathrm{p}$ collisions. Only for the $\mathrm{Be}+\mathrm{Be}$ collisions at $150 \mathrm{~A} \mathrm{GeV} / \mathrm{c}$ the model is not able to provide a description of the data. However, it should be noted that this data point has still a preliminary status, and additional experimental checks are in progress [74].

For the UrQMD simulations of $\mathrm{p}+\mathrm{p}$ reactions, we also present the results for 'most central' p+p events calculated in the samples with $E_{\mathrm{F}}=0$. These results are presented by crosses. The centrality selection performed for $\mathrm{p}+\mathrm{p}$ reactions within the UrQMD simulations leads to essentially smaller values of $\omega_{\mathrm{p}+\mathrm{p}}^{-}$. The 

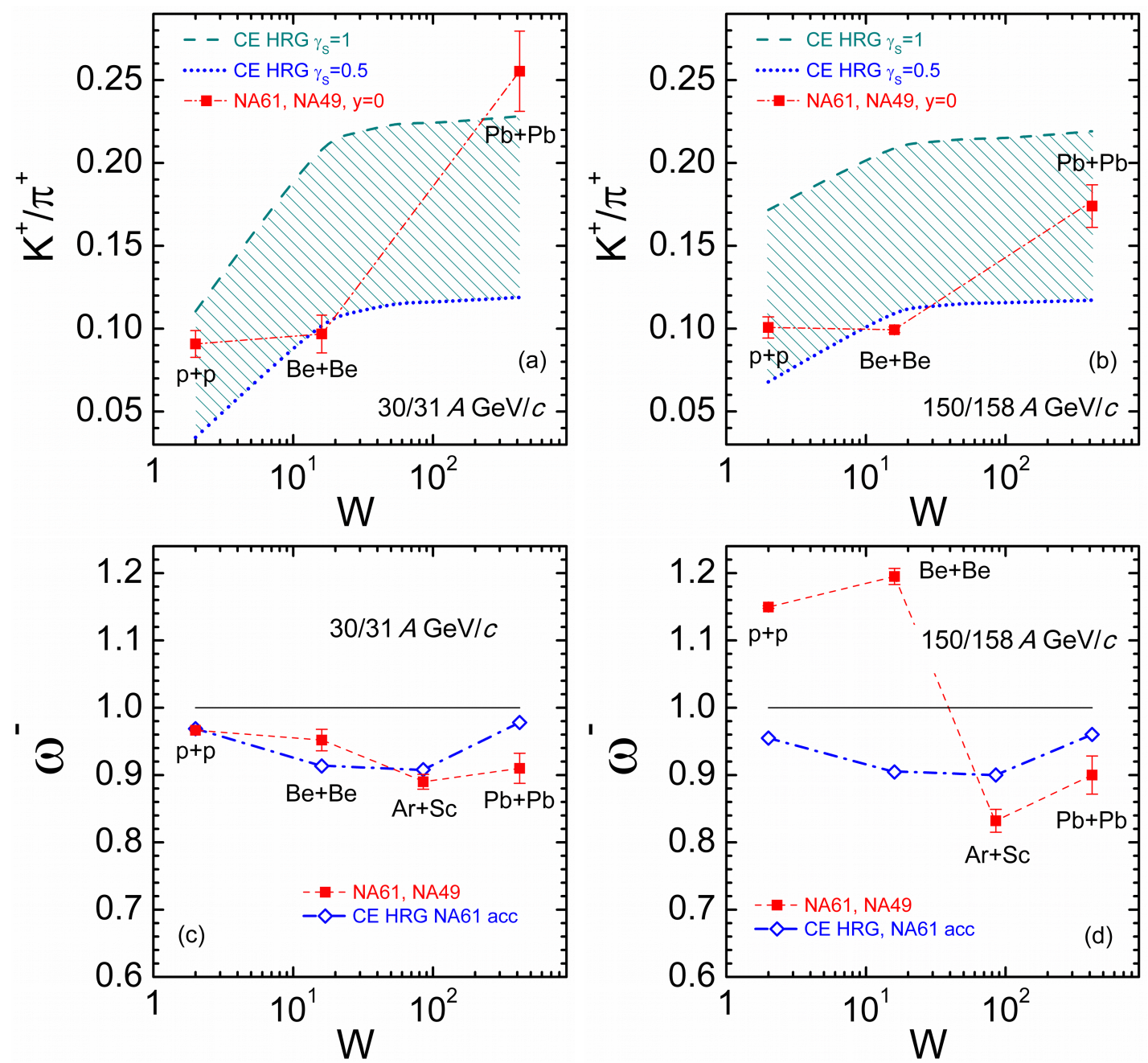

Figure II.6: The CE HRG results for $K^{+} / \pi^{+}(\mathrm{a}-\mathrm{b})$ as functions of $W \equiv A_{1}+$ $A_{2}$ which equals to the total number of nucleons in the colliding nuclei. The calculations are done at for the fireball at the freeze-out stage. The data are shown by red squares for $K^{+} / \pi^{+}[69,70,71]$.

suppression of fluctuations in the $p+p$ collisions due to the centrality selection suggests a monotonic behavior of fluctuations with the system size.

The UrQMD explains the qualitative trends of the $K^{+} / \pi^{+}$ratio. A comparison of the UrQMD results with the data on $\omega^{-}$looks well except for only one point $\mathrm{Be}+\mathrm{Be}$ at $150 \mathrm{~A} \mathrm{GeV} / c$.

Two effects - statistical and dynamical - are identified in the e-by-e fluctuations of hadron production at the SPS energies. Statistical effects are clearly seen in 

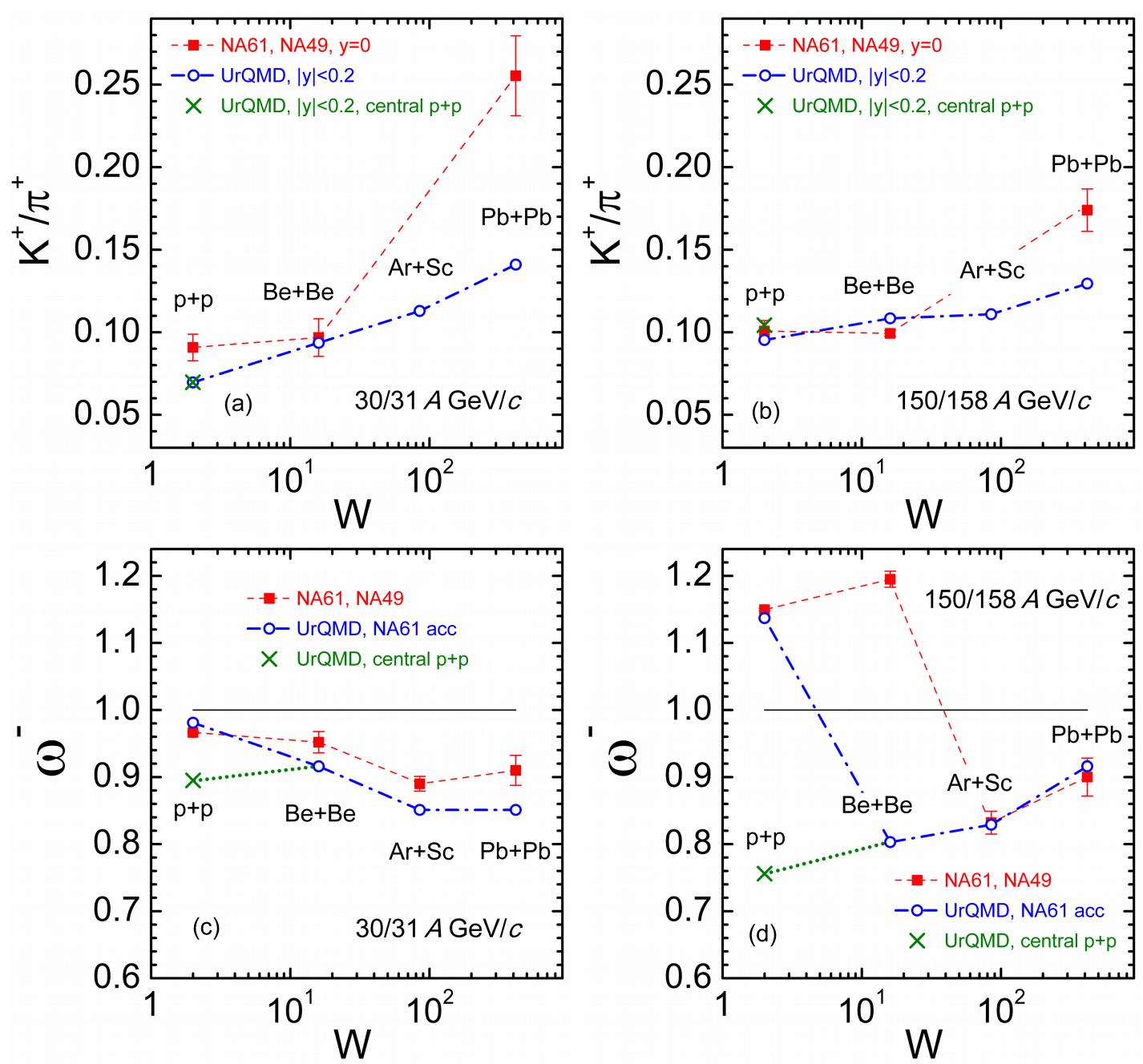

Figure II.7: The data are the same as in Fig. II.6. The UrQMD results are shown by open circles. See text for details. Crosses denote the UrQMD results for the most central $\mathrm{p}+\mathrm{p}$ inelastic collisions.

both CE HRG and UrQMD calculations. The global charge conservation imposed in the HRG by the CE formulation suppresses the particle number fluctuations. This effect is stronger for large collision systems. On the other hand, the dynamical fluctuations are most pronounced in the $p+p$ reactions at high collision energy. The experimental value of the scaled variance $\omega^{-}$becomes larger than unity. The values higher than 1 can not be explained within the CE HRG model. However, the UrQMD is capable to take into account these dynamic effects in $\mathrm{p}+\mathrm{p}$ reactions. The only reaction for which the value of $\omega^{-}$is not described by the UrQMD 
simulation is $\mathrm{Be}+\mathrm{Be}$ at $150 \mathrm{~A} \mathrm{GeV} / c$. Note that the UrQMD results for $\omega^{-}$in this reaction appear to be extremely sensitive to the exact centrality selection procedure.

To clarify the dynamical features of the e-by-e fluctuations, an implementation of the centrality selection procedure in $\mathrm{p}+\mathrm{p}$ reactions is proposed. A comparison of $\mathrm{p}+\mathrm{p}$ and $\mathrm{A}+\mathrm{A}$ collisions should be done with the appropriate centrality selection procedures in both reactions. 


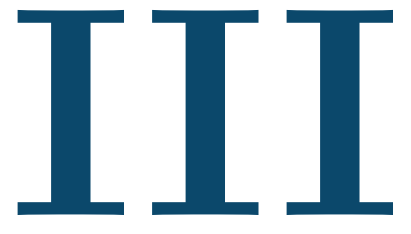

\section{Modeling the hadronic phase of heavy ion collisions by an expanding hadron gas}

The thermal statistical model discussed in the previous chapter provides a surprisingly accurate description of the latest stages of the heavy ion collisions. Only with a handful of thermodynamic parameters the thermal model is able to describe the particle yields at the chemical freeze-out stage of the collision. Though the thermal model does not take into account the complicated non-equilibrium dynamics and interactions of the system, assuming non-interacting Hadron Resonance Gas description, it provides as simple yet convenient tool to analyze heavy ion collisions. The thermal model analysis allows mapping the chemical freeze-out of heavy ion collisions to the QCD phase diagram. However, the chemical freeze-out of the system, the moment when the chemical composition of the system is assumed to be fixed, is not the latest stage of its evolution. The system continues to expand and cool down until the so-called kinetic freeze-out occurs, at this stage, the kinetic equilibrium can not be maintained anymore. The temperature of the kinetic freeze-out is often extracted by blast-wave fits to the $p_{T}$ spectra of stable hadrons. This procedure depends on the assumptions regarding the flow velocity profile and the freeze-out hypersurface. In this chapter, a method to determine the kinetic freeze-out temperature in heavy-ion collisions from the measured yields 
of short-lived resonances is presented. The method is based on a description of heavy ion collisions by an isentropically expanding Hadron Resonance Gas in partial chemical equilibrium. The expansion is modeled between the chemical and kinetic freeze-outs, during this stage, the yields of many short-lived resonances are suppressed. The framework describes the suppression of the yields of many short-lived resonances at $T=T_{\text {kin }}<T_{\mathrm{ch}}$. The values of $T_{\text {kin }}$ and $T_{\mathrm{ch}}$ are extracted for various centralities in $\mathrm{Pb}-\mathrm{Pb}$ collisions at $\sqrt{s_{N N}}=2.76 \mathrm{TeV}$ by fitting the abundances of both the stable hadrons and the short-lived resonances like $\rho^{0}$ and $\mathrm{K}^{* 0}$, that were measured by the ALICE collaboration. This allows to extract the kinetic freeze-out temperature from the measured hadron and resonance yields alone, independent of assumptions about the flow velocity profile and the freeze-out hypersurface. The extracted $T_{\text {ch }}$ values exhibit a moderate multiplicity dependence whereas $T_{\text {kin }}$ drops, from $T_{\text {kin }} \simeq T_{\text {ch }} \simeq 155 \mathrm{MeV}$ in peripheral collisions to $T_{\text {kin }} \simeq 110 \mathrm{MeV}$ in $0-20 \%$ central collisions. Predictions for other short-lived resonances are presented.

This chapter is based on the research performed by the author of this thesis and published in Ref.[7].

\section{III.1 Hadronic phase in heavy ion collisions}

The abundances of stable hadrons measured in relativistic heavy-ion experiments at the Schwerionen Synchrotron (SIS), the Super Proton Synchrotron (SPS), the Relativistic Heavy Ion Collider (RHIC), and the Large Hadron Collider (LHC) agree quite well with thermal model calculations which are based on the assumption of chemical freeze-out at temperature $T_{\text {ch }} \simeq 150-160 \mathrm{MeV}[75,76,21,77]$. However, the thermal model significantly overpredicts the yields of short-lived resonances, like $\mathrm{K}^{*}$ or $\rho[78,79,80,81]$, this indicates a presence of an additional mechanism that suppresses these yields. This suppression of resonances is usually attributed to the existence of a hadronic phase, which properties are usually estimated by analyzing particle $p_{T}$ spectra which are measured in detail at experiment $[82,77]$ for both, the long-lived and short-lived hadrons. The analysis of the measured particle spectra suggests that the expanding system seems to be in kinetic (but not in chemical) equilibrium. This kinetic equilibrium is maintained during the cooling after the chemical freeze-out down to a kinetic freeze-out temperature $T_{\text {kin }}<T_{\text {ch }}$. The common way to estimate the kinetic freeze-out temperature is by 
performing blast-wave fits to the $p_{T}$ spectra of stable hadrons. This procedure assumes an interplay of a particular flow velocity profile and a kinetic freeze-out hypersurface. Usually, a cylindrically-symmetric blast-wave model is sufficient to describe the expansion of the fireball [83], which yields $T_{\text {kin }} \sim 100 \mathrm{MeV}$ for the most central collisions at LHC [84], RHIC [77], and SPS [85]. However, different freeze-out geometries can lead to different conclusions [86]. This chapter suggests a novel approach to extract $T_{\text {kin }}$ by simulating the fireball expansion in the hadronic phase. The method, contrary to blast-wave fits, is independent of assumptions about the flow velocity profile and the freeze-out hypersurface.

\section{III.2 Partial chemical equilibrium in Hadron Resonance Gas}

The experimentally observed survival of stable hadron yields and the suppression of the resonance yields during the fireball expansion can be explained by rescattering in the hadronic phase. While the system expands in the hadronic phase the resonances decay and their decay products are rescattered [87, 88, 89]. After that these short-lived resonances can no longer be identified in invariant mass measurements. Hence, this looks like the "observed" resonance yields are suppressed. This scenario was used earlier to estimate the lifetime of the hadronic phase at RHIC and SPS energies from the measured resonance abundances [90, 91, 92], neglecting the effect of resonance regeneration. However, the resonance regeneration plays a significant role in a gas of hadrons, since many elastic hadronic reactions do form intermediate short-lived resonance states, this is reflected in meson-meson and meson-baryon cross-sections [93]. Common examples are $\pi \pi \rightarrow \rho \rightarrow \pi \pi, \pi \mathrm{K} \rightarrow \mathrm{K}^{*} \rightarrow \pi \mathrm{K}$, and $\pi \mathrm{N} \rightarrow \Delta \rightarrow \pi \mathrm{N}$. Rescattering of a resonance decay product in a hadronic fireball is likely to regenerate a resonance. This picture was verified in microscopic transport calculations [89] where it was proved that in the hadronic phase the resonance-formations dominate in pure elastic meson-meson and meson-baryon rescatterings.

The resonance-forming pseudo-elastic reactions obey the law of mass action during the hadronic phase. The kinetic equilibrium in an expanding system is maintained by these reactions, and the conditions of this scenario can be summarized as follows: 


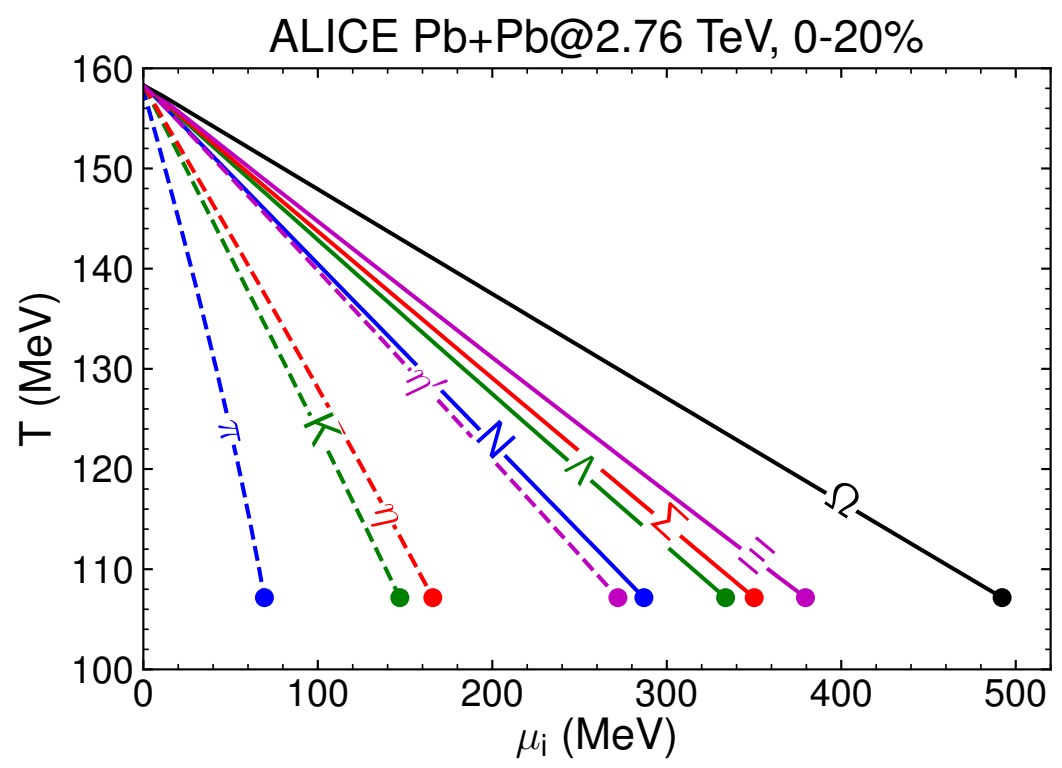

Figure III.1: Temperature dependence of effective chemical potentials of stable hadrons in the HRG in PCE approach. The trajectories describe the evolution in the hadronic phase for the ALICE $\mathrm{Pb}+\mathrm{Pb}$ most central data, the trajectories star at the chemical freeze-out and end at the kinetic freeze-out which is marked by circles. The baryons are depicted by solid and mesons by dashed lines.

- After the chemical freeze-out occurs at $T=T_{\text {ch }}$ the inelastic reactions drive the system out of the chemical equilibrium. The nonequilibrium is maintained in such a way that the total yields of all stable hadrons are frozen. This corresponds to the sum of the primordial yields of table hadrons and their yields that stem from decays of short-lived resonances.

- After the chemical equilibrium is lost, the system expands isentropically until the kinetic freeze-out temperature $T_{\text {kin }}<T_{\mathrm{ch}}$ is reached. This stage is identified with the hadronic phase. It is modeled by the concept of partial chemical equilibrium (PCE) [94]. The resonance decays and regenerations obey the law of mass action, hence the abundances of the different resonances stay in equilibrium with their decay products.

- After the kinetic freeze-out at $T=T_{\text {kin }}$ the resonance regeneration ceases to occur and the resonance decay products do not rescatter. At this point, the resonance abundances at $T=T_{\text {kin }}$ are identified with those measured 
experimentally. This implies that the chemical freeze-out of short-lived resonances coincides with the kinetic freeze-out of bulk hadron matter.

Of course, the actual decoupling of particles in an expanding system is a continuous process that takes place over a range of temperatures. In that sense, the $T_{\text {ch }}$ and $T_{\text {kin }}$ temperatures characterize average conditions for the chemical and kinetic freeze-outs.

The description suggested above is generally consistent with the pioneering ideas regarding strangeness production in heavy-ion collisions [95]. The thermodynamics of the matter in the hadronic phase of the fireball is described here using a hadron resonance gas (HRG) model in PCE [94, 96, 97, 98, 99]. The effective chemical potentials $\tilde{\mu}_{j}$ of all species can be obtained from the condition of equilibrium between the particle and its decay products, and thus are given by:

$$
\tilde{\mu}_{j}=\sum_{i \in \text { stable }}\left\langle n_{i}\right\rangle_{j} \mu_{i}
$$

The index $i$ runs over all particles, whose final abundances are frozen at $T=T_{\mathrm{ch}}$. These hadrons are the ones stable w.r.t. strong decays, i.e. $\pi, \mathrm{N}, \eta, \eta^{\prime}, \mathrm{K}, \Lambda, \Sigma$ 's, $\Xi$ 's, $\Omega$, as well their antiparticles ${ }^{(1)} \cdot \mu_{i}$ are the chemical potentials of particles considered stable. $\left\langle n_{i}\right\rangle_{j}$ is the mean number of hadron species $i$ resulting from decays of hadron species $j$. Then the system evolution in PCE follows from the conditions of the conservation of the total yields of the stable hadrons and constant entropy per baryon, i.e. isentropic expansion:

$$
\begin{aligned}
\sum_{j \in \mathrm{hrg}}\left\langle n_{i}\right\rangle_{j} n_{j}\left(T, \tilde{\mu}_{j}\right) V & =N_{i}^{\mathrm{tot}}\left(T_{\mathrm{ch}}\right), \quad i \in \text { stable } \\
\sum_{j \in \mathrm{hrg}} s_{j}\left(T, \tilde{\mu}_{j}\right) V & =S\left(T_{\mathrm{ch}}\right) .
\end{aligned}
$$

The description of the system can then be obtained after solving the above equations. The solution will provide the chemical potentials $\mu_{j}$ and the system volume $V$ during the system's expansion. The index $j$ runs over all hadrons and resonances in the list, $n_{j}$ and $s_{j}$ are the grand-canonical number- and entropy densities of the hadron species $j$ in the multi-component ideal hadron gas, $N_{i}^{\text {tot }}\left(T_{\text {ch }}\right)$ and $S\left(T_{\mathrm{ch}}\right)$ are, respectively, the total yield of stable hadron species $i$ and the

\footnotetext{
(1) Alternatively, one can treat the yields of long-lived resonances such $\phi, \omega, \Xi(1530)$, and/or $\Lambda(1520)$ to be frozen at $T=T_{\text {ch }}$ as well [99]. We verified that the results presented here look very similar in such a scenario.
} 
total entropy of the system during the whole expansion. In the following, the energy-dependent Breit-Wigner (eBW) scheme is used for modeling the spectral functions of all resonances [100]. The hadron branching ratios are assumed to be energy independent. $\left\langle n_{i}\right\rangle_{j}$ is evaluated using the PDG branching ratios. Excluded-volume and strangeness undersaturation effects are omitted unless stated otherwise. The calculations are performed using the open-source Thermal-FIST package [54], which contains a numerical implementation of the PCE-HRG model defined above (available since version 1.2.6 via [101]).

The Equations (III.2) and (III.3) can be solved numerically to obtain the temperature dependence of the volume $V$ and of the chemical potentials $\tilde{\mu}_{j}$ of all species during the hadronic phase III.1. Within the proposed PCE-HRG model, this dependence was presented earlier in Ref. [102] for the LHC energies. During the cooling, the yields of short-lived resonances, such as $\mathrm{K}^{*} / \mathrm{K}$ and $\rho / \pi$, are not conserved while the system is in the hadronic phase. They decrease as the system expands, their values at $T=T_{\text {kin }}$ are suggested to describe the suppression seen in measurements, as first predicted in Ref. [103] before precision data were available. This method of relating the resonance suppression to the duration of the hadronic phase is suggested to extract the kinetic freeze-out temperature from experimental data.

\section{III.3 Kinetic freeze-out temperature from yields of short-lived resonances}

Here the above approach is presented for a determination of the kinetic freeze-out temperature of $2.76 \mathrm{TeV} \mathrm{Pb}-\mathrm{Pb}$ collisions at the LHC. This is done by performing PCE-HRG model fits to the measured yields of pions, kaons, protons, $\Lambda, \Xi, \Omega$, $\phi, \mathrm{K}_{0}^{S}, \mathrm{~K}^{* 0}$, and $\rho^{0}$, of the ALICE collaboration, for $0-20 \%, 20-40 \%, 40-60 \%$, and $60-80 \%$ centralities $[82,104,105,80,81]$. It should be noted that the yields are symmetrized between particles and antiparticles, i.e., we assume $\mu_{B}=0$. The fitting procedure assumes three fit parameters: the chemical freeze-out temperature $T_{\mathrm{ch}}$ and volume $V_{\mathrm{ch}}$, and the kinetic freeze-out temperature $T_{\text {kin. }}$. As compared to the conventional chemical freeze-out fit, this procedure employs one additional parameter $T_{\text {kin }}$ to describe the yields of short-lived resonances. The final yields of all species are evaluated at $T=T_{\mathrm{kin}}$. The single freeze-out scenario, $T_{\mathrm{kin}}=T_{\mathrm{ch}}$, is also analyzed. All abundances of all species are, in this single freeze-out scenario, 
Table III.1: Values of the PCE-HRG model thermal fit parameters to ALICE data for $\sqrt{s_{N N}}=2.76 \mathrm{TeV} \mathrm{Pb}-\mathrm{Pb}$ collisions at different centralities. For each centrality the first row corresponds to the single freeze-out scenario, the second row corresponds to separate chemical and kinetic freeze-outs scenario.

\begin{tabular}{cccc}
\hline \hline Centrality & $T_{\text {ch }}(\mathrm{MeV})$ & $T_{\text {kin }}(\mathrm{MeV})$ & $\chi^{2} /$ dof \\
\hline $0-20 \%$ & $160.2 \pm 3.1$ & - & $23.6 / 8$ \\
& $158.3 \pm 2.8$ & $107.1 \pm 8.2$ & $10.5 / 7$ \\
\hline $20-40 \%$ & $162.9 \pm 3.1$ & - & $19.5 / 8$ \\
& $161.7 \pm 2.9$ & $117.3 \pm 10.8$ & $12.8 / 7$ \\
\hline $40-60 \%$ & $162.3 \pm 3.0$ & - & $12.5 / 8$ \\
& $161.8 \pm 2.9$ & $131.2 \pm 15.9$ & $10.6 / 7$ \\
\hline $60-80 \%$ & $155.5 \pm 2.5$ & - & $19.1 / 8$ \\
& $155.5 \pm 2.5$ & $155.5_{-24.5}^{+2.5}$ & $19.1 / 7$ \\
\hline \hline
\end{tabular}

described by the chemical equilibrium ideal HRG model. The PCE-HRG fit procedure described above has been implemented in Thermal-FIST since version 1.2.6 and can be obtained via Ref. [101].

The results of the performed fits are exhibited in Table III.1. The centrality dependencies of both $T_{\text {kin }}$ and $T_{\text {ch }}$ are shown in Fig. III.2 as a function of the charged particle multiplicity $d N_{\mathrm{ch}} / d \eta$ [106]. The parameter errors of the performed fits were obtained by analyzing the $\chi^{2}$ profiles, see e.g. $\chi^{2}$ profiles as functions of $T_{\text {kin }}$ in Fig. III.3. The error bar of $T_{\text {kin }}$ is asymmetric for the $60-80 \%$ centrality because of the restriction $T_{\text {kin }} \leq T_{\mathrm{ch}}$.

As discussed before, the single freeze-out scenario cannot describe simultaneously the yields of stable hadrons and short-lived resonances in central collisions. The yields of short-lived $\mathrm{K}^{* 0}$ and $\rho^{0}$ mesons are significantly overestimated by the model with $T_{\text {ch }}=T_{\text {kin }} \simeq 155 \mathrm{MeV}$. This overestimation becomes milder in peripheral collisions, where the suppression of the resonance yields is measured to be not so strong. The separation of kinetic and chemical freeze-outs leads to an improved description of the measured yields for all centralities, except for the most peripheral bin where the suppression is almost absent. $T_{\text {ch }}$ shows only a slight centrality dependence, suggesting a consistent and universal value in the $155-160 \mathrm{MeV}$ range throughout. The extracted kinetic temperature increases monotonically from $T_{\text {kin }} \simeq 110 \mathrm{MeV}$ for the $0-20 \%$ centrality bin, to $T_{\text {kin }} \simeq T_{\text {ch }}=155 \mathrm{MeV}$ for 


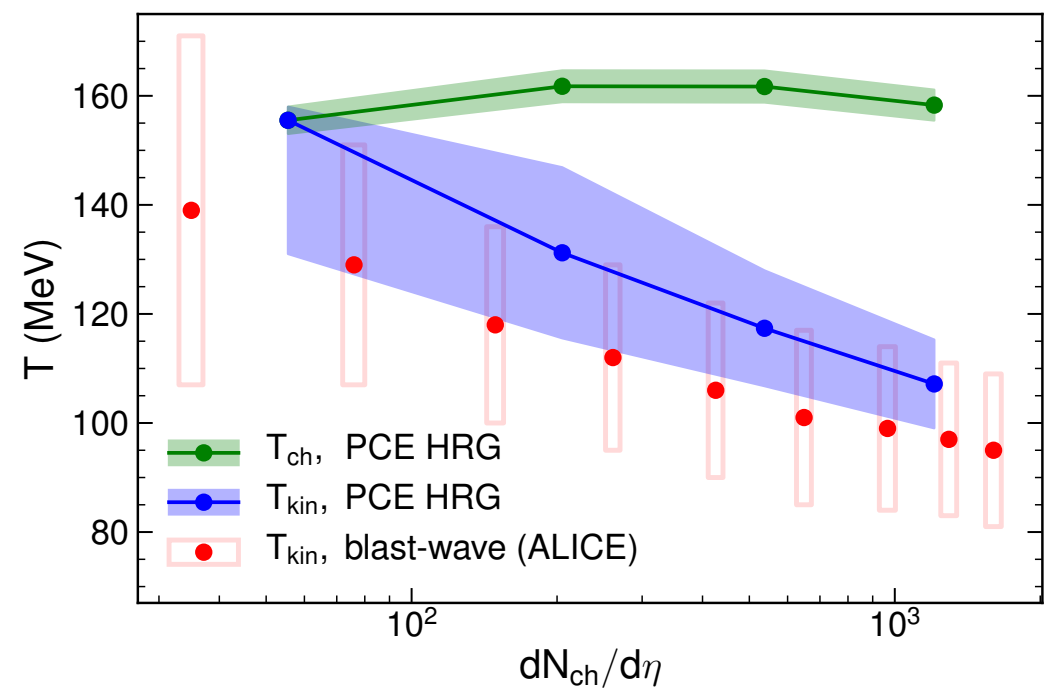

Figure III.2: The values of the chemical $T_{\mathrm{ch}}$ (green symbols) and kinetic $T_{\text {kin }}$ (blue symbols) freeze-out temperatures extracted from the PCE-HRG model fits to the ALICE collaboration data on the production of hadrons and resonances in $\mathrm{Pb}+\mathrm{Pb}$ collisions at $\sqrt{s_{\mathrm{NN}}}=2.76 \mathrm{TeV}$ for various centralities, depicted as a function of charged multiplicity. The red symbols depict the $T_{\text {kin }}$ values extracted from blast-wave fits to the $p_{T}$ spectra of pions, kaons, and protons in Ref. [82].

$60-80 \%$ centrality. These results point out the existence of a hadronic phase in heavy-ion collisions with a lifetime that depends on the geometry of the collision, a rather long-lived one in central collisions and a short-lived one in peripheral collisions.

Let us compare these results to the $T_{\text {kin }}$ values resulting from blast-wave model fits [83] to the $p_{T}$ spectra of pions, kaons, and protons, as presented by the ALICE collaboration in Ref. [82] (red symbols in Fig. III.2). The predictions of the proposed PCE-HRG model are in a fair agreement with this analysis, although the $T_{\text {kin }}$ values of Ref. [82] are on the lower side of our error bands. Recent blast-wave model studies $[107,108]$ take into account the resonance feeddown and the outcoming modifications of the $p_{T}$ spectra. The $T_{\text {kin }}$ values calculated in Ref. [107] lie considerably closer to $T_{\text {ch }}$ than in the present study, whereas Ref. [108] predicts a much smaller value $T_{\text {kin }} \simeq 80 \mathrm{MeV}$ for most central collisions. The present in the literature large spread of the $T_{\text {kin }}$ values is an indication of significant systematic uncertainties which are currently present in the blast-wave model approach. It should be noted that none of those above three analyses does 


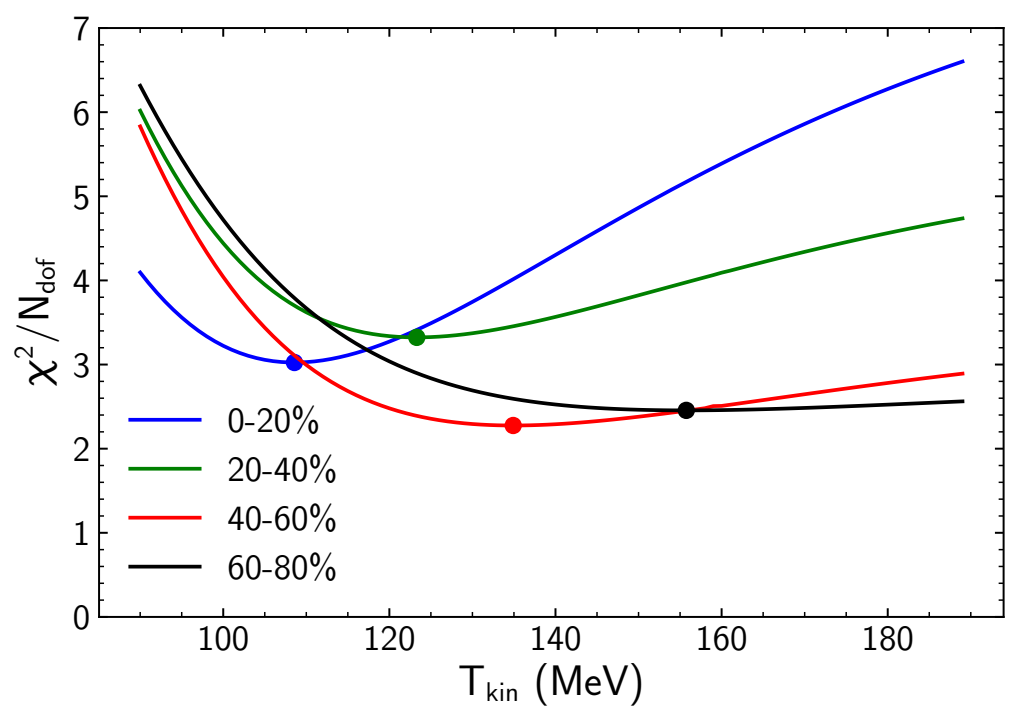

Figure III.3: The $\chi^{2}$ profiles of the fits from Fig. III.2 as functions of the kinetic $T_{\text {kin }}$ freeze-out temperature. The profiles were obtained by fitting the chemical $T_{\text {ch }}$ freeze-out temperature and the freeze-out volume $V$ at fixed $T_{\text {kin }}$.

incorporate constraints from the data on short-lived resonances, in contrast to the study presented here. Thus, the inclusion of the measured spectra of resonances is one way to improve the blast-wave approach. The $p_{T}$ spectra fit also depend on the validity of the blast-wave model's assumed flow velocity profile and freeze-out hypersurface. The concept presented here is free of this issue.

The uncertainties arising from the implementation of the HRG model deserve separate attention. The sensitivity to the modeling of spectral functions was estimated by considering, additionally to the eBW scheme, the zero-width treatment of resonances. The extracted $T_{\mathrm{ch}}$ and $T_{\text {kin }}$ values are, respectively, about $2-3 \mathrm{MeV}$ smaller and $5 \mathrm{MeV}$ larger in the zero-width case than in the eBW case. The fit quality worsens for all centralities (except for the most peripheral bin). This is mainly a consequence of the increased proton yield in the zero-width scheme. An incomplete strangeness equilibration in the HRG model can be studied by introducing a strangeness saturation parameter, $\gamma_{S} \leq 1[95,109]$. This influences significantly the most peripheral $(60-80 \%)$ bin only. Here, $\gamma_{S} \simeq 0.85$, and the extracted $\chi^{2}$ value decreases by about a factor of two, while $T_{\text {ch }}$ increases to about $160 \mathrm{MeV}$. This is in line with previous statistical model analyses of the LHC data $[110,111,112]$. Separate chemical freeze-outs of strange and non-strange hadrons is another possibility that has been discussed [113, 114]. 


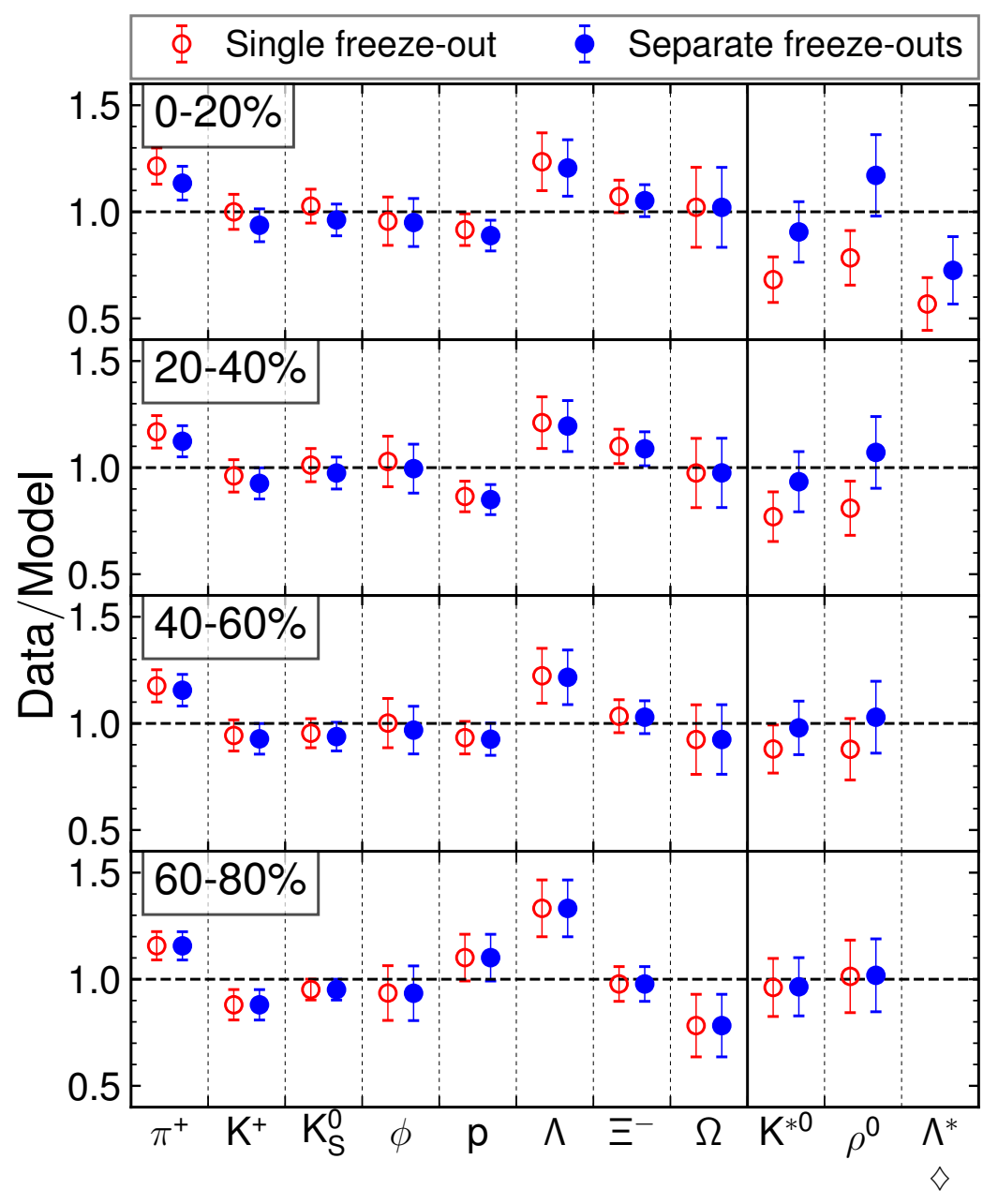

Figure III.4: The data/model ratios resulting from thermal fits to particle yields measured in $\mathrm{Pb}-\mathrm{Pb}$ collisions of various centrality at $\sqrt{s_{N N}}=2.76 \mathrm{TeV}$. Fits are performed within the single freeze-out HRG picture (open red circles), and the separate freeze-outs PCE-HRG picture (full blue circles). Here $\Lambda^{*}$ corresponds to the $\Lambda(1520)$. The $\Lambda(1520)$ yields were not used in the fit procedure.

The HRG model is often extended by considering repulsive interactions via excluded volume corrections, this can have a sizable influence on thermal fits to the data $[115,116,117]$. A moderate excluded volume correction is considered here by repulsive (anti)baryon-(anti)baryon interactions with a baryonic eigenvolume parameter $b \simeq 1 \mathrm{fm}^{3}$. This is motivated by the analysis of lattice QCD data on baryon number susceptibilities [1] and Fourier coefficients [118]. The excluded-volume PCE-HRG model fits slightly change $T_{\mathrm{ch}}$ and $T_{\text {kin }}$ values which 
are, respectively, about 2-3 MeV larger and 2-3 MeV smaller than in the ideal HRG case. The changes in the $\chi^{2}$ values are insignificant.

The particle list of the thermal HRG model is often extended by light nuclei to consider their yields in the analysis of experimental data [119]. Within the PCE-HRG framework, one can either treat the nuclei as stable species whose yields are frozen at $T_{\mathrm{ch}}$ or one accepts that these loosely bound objects, as compared to the temperatures available, can be destroyed and regenerated during the evolution in the hadronic phase. In the latter case, the nuclear abundances are in equilibrium with the abundances of their constituents, as follows from the Saha equation (see Ref. [102] for details). We verified that the available data on light nuclei production in $0-20 \%$ central $\mathrm{Pb}-\mathrm{Pb}$ collisions are well described in both scenarios.

The presented analysis can be further extended to study potentially measurable particles. Within the PCE-HRG model, it is interesting to test the sensitivity of other unstable hadrons to the value of kinetic freeze-out temperature $T_{\text {kin. }}$. This can be done by analyzing resonance-to-stable hadron yield ratios. Specifically, the behavior of ratios $\phi / \pi, \omega / \pi, \rho / \pi, \Delta^{++} / \mathrm{p}, \mathrm{K}^{* 0} / \mathrm{K}^{-}, f_{0}(980) / \pi$, $\Sigma(1385) / \Lambda, \Lambda(1520) / \Lambda, \Xi(1530)^{0} / \Xi$, and $\Xi(1820) / \Xi$, is studied here at the LHC conditions $\left(\mu_{B}=0\right)$. Given that the obtained chemical freeze-out temperature is in general independent on the centrality, here we fix $T_{\text {ch }}=155 \mathrm{MeV}$ and study the dependence of the ratios on $T_{\text {kin }}$ only. It was checked that the results are not sensitive to the specific value of $T_{\mathrm{ch}}$, e.g. $T_{\mathrm{ch}}=160 \mathrm{MeV}$ giving very similar results. All presented ratios here are normalized by their values at $T_{\mathrm{ch}}=155 \mathrm{MeV}$ to eliminate the influence of effects not related to the hadronic phase dynamics. These double ratios quantify the suppression of resonance yields in (semi-)central collisions, where $T_{\text {kin }}<T_{\text {ch }}$, relative to the most peripheral collisions (or, alternatively, to a pp/pA baseline), where $T_{\text {kin }} \simeq T_{\text {ch }}$. The $T_{\text {kin }}$ dependence of the above-listed double ratios is depicted in Fig. III.5.

In general, different resonance particles interact differently in the medium and thus can have different freeze-out temperatures. The effect of the hadronic phase on the resonances can be quantified by the temperature dependence in Fig. III.5. The yields of long-lived resonances $\phi(\tau \approx 46 \mathrm{fm} / c)$ and $\Xi(1530)(\tau \approx 22 \mathrm{fm} / c)$ change little in the PCE-HRG approach for $T_{\text {kin }} \gtrsim 100 \mathrm{MeV}$, even though this scenario assumes equilibrium of these long-lived resonances with their decay products. Hence, an absence of suppression of long-lived resonance yields, which does not necessarily imply that these objects do not interact after the chemical 
freeze-out. The long-lived $\omega$ meson $(\tau=23 \mathrm{fm} / c)$, on the other hand, would be notably suppressed in central collisions if it interacts in the hadronic phase.

The short-lived $\rho^{0}(\tau=1.3 \mathrm{fm} / c)$ and $\mathrm{K}^{* 0}(\tau=4.2 \mathrm{fm} / c)$ meson yields exhibit a significant suppression as $T_{\text {kin }}$ decreases, as elaborated earlier. On the other hand, the yields of short-lived baryonic resonances $\Delta^{++}(\tau=1.7 \mathrm{fm} / \mathrm{c})$ and $\Sigma(1385)(\tau=5 \mathrm{fm} / c)$ change only mildly. For $T_{\text {kin }}=100 \mathrm{MeV}$ one observes only a $10-15 \%$ suppression in the $\Delta^{++}$yields and virtually no change for $\Sigma(1385)$. Thus, if the slight system size dependence of the yield ratios involving these resonances will indeed be observed experimentally, such an observation cannot be interpreted as evidence against the existence of a long-lived hadronic phase. The presented observations are qualitatively consistent with prior results of Monte Carlo simulations of heavy-ion collisions where the hadronic afterburner UrQMD [87] was employed. The result for $\Sigma(1385) / \Lambda$ is also in line with a weak system-size dependence of this ratio as was observed experimentally at RHIC for $\sqrt{s_{N N}}=$ $200 \mathrm{GeV}[121]$.

A particularly interesting case is the scalar $f_{0}(980)$ meson. The nature of $f_{0}(980)$ is not established and its lifetime is not constrained. The PDG listing [93] gives a loose bound on the mass width $\Gamma_{f_{0}} \sim 10-100 \mathrm{MeV}$. This corresponds to a lifetime between about 2 and $20 \mathrm{fm} / c$. For the lower bound, the lifetime is shorter than the lifetime of the hadronic phase, hence the PCE-HRG model assumption of detailed balance between decays and regenerations of $f_{0}(980)$ is valid. If this is the case and the lifetime of $f_{0}(980)$ is much shorter than the lifetime of the hadronic phase then the $f_{0}(980) / \pi$ ratio will be significantly suppressed as presented in Fig. III.5, there the ratio drops by about a factor three for $T_{\text {kin }}=100 \mathrm{MeV}$. For the upper bound of the $f_{0}(980)$ lifetime, it is more reasonable to expect that its yield is frozen at $T_{\mathrm{ch}}$ and will not be modified significantly in the hadronic phase. The measurements of the $f_{0}(980) / \pi$ ratio in heavy-ion collisions at different centralities in the highest energy heavy ion collisions at the LHC (or RHIC) can thus provide indirect information on its lifetime: If the suppression of the $f_{0}(980) / \pi$ ratio in central collisions relative to peripheral ones will be observed, this will be evidence for a short $f_{0}(980)$ lifetime. An absence of such suppression, on the other hand, favors a large $f_{0}(980)$ lifetime.

To summarize, the above-presented approach can be used as a novel method to extract the kinetic freeze-out temperature in heavy-ion collisions based on the yields of short-lived resonances. This method, which assumes expanding hadron 
resonance gas in partial chemical equilibrium, does not require additional input such as the flow velocity profile and the freeze-out hypersurface. In this sense, the PCE-HRG approach to extracting $T_{\text {kin }}$ is advantageous to the commonly adopted fits to the $p_{T}$ spectra. The analysis of ALICE data on $\mathrm{Pb}-\mathrm{Pb}$ collisions at the LHC yields a moderate multiplicity dependence of $T_{\mathrm{ch}}$ whereas the kinetic freeze-out temperature drops from $T_{\text {kin }} \simeq T_{\text {ch }} \simeq 155 \mathrm{MeV}$ in peripheral collisions to $T_{\text {kin }} \simeq 110 \mathrm{MeV}$ in $0-20 \%$ most central collisions. This result is in qualitative agreement with prior studies employing the blast-wave model fits.

An interesting observation is that not all short-lived resonances are necessarily suppressed in a long-lasting hadronic phase: In contrast to $\rho^{0}$ and $\mathrm{K}^{* 0}$ mesons, the yields of baryon resonances $\Delta^{++}$and $\Sigma(1385)$ change slightly in the hadronic phase. We point out a possibility to constrain the lifetime of $f_{0}(980)$ meson: A (non)observation of a suppressed $f_{0}(980) / \pi^{ \pm}$ratio in central collisions favors a long (short) $f_{0}(980)$ lifetime. Studies within this framework can be extended further to lower collision energies, and to analyze other sensitive probes of freezeout dynamics and properties of the hadronic matter, such as fluctuations and correlations of identified hadron numbers [122, 123]. 


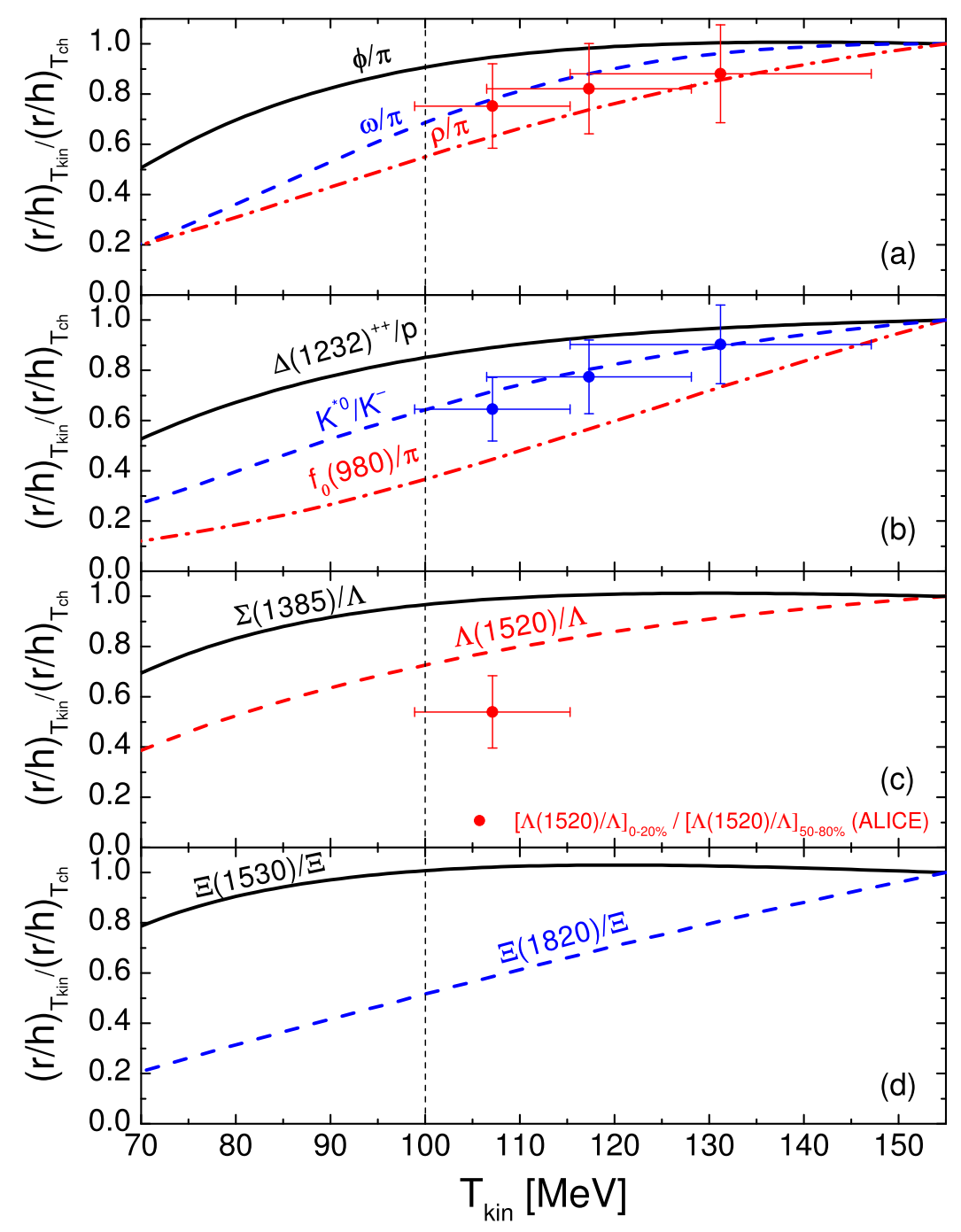

Figure III.5: Dependence of the yield ratios (a) $2 \phi /\left(\pi^{+}+\pi^{-}\right)$(solid black line), $2 \omega /\left(\pi^{+}+\pi^{-}\right)$(dashed blue line), and $2 \rho^{0} /\left(\pi^{+}+\pi^{-}\right)$(dot-dashed red line), (b) $\Delta^{++} / \mathrm{p}$ (solid black line), $\mathrm{K}^{* 0} / \mathrm{K}^{-}$(dashed blue line), and $2 f_{0}(980) /\left(\pi^{+}+\pi^{-}\right)$(dotdashed red line), (c) $\Sigma(1385) / \Lambda$ (solid black line) and $\Lambda(1520) / \Lambda$ (dashed red line), and (d) $\Xi(1530) / \Xi$ (solid black line) and $\Xi(1820) / \Xi$ (dashed blue line) on the kinetic freeze-out temperature $T_{\text {kin }}$. The ratios are normalized to their values at $T=T_{\mathrm{ch}}=155 \mathrm{MeV}$. The points in (a) and (b) depict, respectively, the experimental data for the ratios of $2 \rho^{0} /\left(\pi^{+}+\pi^{-}\right)$and $\mathrm{K}^{* 0} / \mathrm{K}^{-}$in $0-20 \%$, $20-40 \%$, and $40-60 \%$ relative to the ones in $60-80 \% \mathrm{~Pb}-\mathrm{Pb}$ collisions at $\sqrt{s_{N N}}=$ $2.76 \mathrm{TeV}$. The red point in (c) depicts ALICE collaboration data [120] for the ratio of $\Lambda(1520) / \Lambda$ measured in $0-20 \%$ to the one in $50-80 \% \mathrm{~Pb}-\mathrm{Pb}$ collisions at $\sqrt{s_{N N}}=2.76 \mathrm{TeV}$. The dashed vertical line corresponds to a $T_{\text {kin }}=100 \mathrm{MeV}-\mathrm{a}$ typical value for the kinetic freeze-out temperature in most central collisions. 


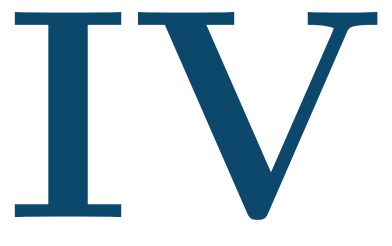

\section{Repulsive interactions between baryons in a hadron gas}

The previous chapters were related to the hadronic evolution of the heavy ion collisions. This chapter is related to static hadronic matter with repulsive interactions. The repulsive interactions are modeled by assigning finite volume to baryons. This is done by applying the Beth-Uhlenbeck (BU) formalism for repulsive hard-core interactions between baryons. The BU approach is also compared to the classical van der Waals excluded volume model which neglects quantum effects. The second virial coefficient $a_{2}$ - the "excluded volume" parameter, calculated within the BU approach is found to be temperature-dependent, and it differs dramatically from the classical EV model result. At temperatures $T=100-200 \mathrm{MeV}$, the widely used classical EV model underestimates the EV parameter for nucleons at a given value of the nucleon hard-core radius by large factors of 3-4. Previous studies, which employed the hard-core radii of hadrons as an input into the classical EV model, have to be re-evaluated using the appropriately rescaled EV parameters. The BU approach is used to model the repulsive baryonic interactions in the hadron resonance gas (HRG) model. Lattice data for the second and fourth-order net baryon susceptibilities are described fairly well when the temperature dependent BU baryonic excluded volume parameter corresponds to nucleon hard-core radii of $r_{c}=0.25-0.3 \mathrm{fm}$. The role of the attractive baryonic interactions is also 
considered. It is argued that the HRG model with a constant baryon-baryon EV parameter $v_{N N} \simeq 1 \mathrm{fm}^{3}$ provides a simple yet efficient description of baryon-baryon interaction in the crossover temperature region.

This chapter is based on the research performed by the author of this thesis and published in Ref.[1].

\section{IV.1 Classical excluded-volume model}

In a gas of parties the short-rage repulsive interactions can be modeled within the classical EV model where the available system volume is reduced by the particle finite volume $v$, i.e. $V \rightarrow V-v N$, where $N$ is the total number of particles [124, $125,126,127,128,129,130]$. The repulsive interactions transform the system energy $E$ in the Grand Canonical Ensemble as:

$$
E=-p V+s T+\mu N \rightarrow E=-p V+s T+(\mu-p v) N
$$

which introduces the effective chemical potential $\mu^{*}=\mu-p v$. In the Canonical Ensemble this substitution results in the well known van der Waals equation of state

$$
p^{\mathrm{ev}}(T, n)=\frac{T n}{1-v n}
$$

in which the attractive van der Waals interactions are omitted. Here $n \equiv N / V$ is the particle number density.

The pressure function, $p(T, n)$, can be expanded in a series where $k$-th term is proportional to the particlle density to the power of $k$, i.e. $n^{k}$, this is the virial expansion [131, 132, 133]

$$
p(T, n)=T \sum_{k=1}^{\infty} a_{k}(T) n^{k} .
$$

Here $a_{k}$ are the virial coefficients. From Eq. (IV.2) it follows that in the classical EV model these virial coefficients are temperature independent:

$$
a_{k}^{\mathrm{ev}}=v^{k-1}
$$

From the second virial coefficient a hard-core radius $r_{c}$ of a given constituent can be extracted. In an approximation where quantum mechanical effects are 
neglected, 2-body interaction potential can be related to $a_{2}(T)$ by

$$
a_{2}(T)=\frac{1}{2} \int d^{3} r\left\{1-\exp \left[-\frac{U(r)}{T}\right]\right\} .
$$

A repulsive hard-core potential $U(r)$ is a barrier with an infinite wall placed at $r=2 r_{c}$ :

$$
U(r)= \begin{cases}\infty, & \text { if } r<2 r_{c} \\ 0, & \text { if } r>2 r_{c} .\end{cases}
$$

Substituting $U(r)$ from (IV.6) into (IV.5) yields

$$
a_{2}^{\mathrm{ev}}=v=\frac{16 \pi r_{c}^{3}}{3}
$$

On the other side, the pressure of an interacting thermodynamic system can be expressed via the Mayer's cluster expansion. This expansion is in terms of the powers of the fugacity, $\lambda=e^{\mu / T}$. It will be used below for the comparison with the Beth-Uhlenbeck approach. The cluster expansion is written as $[131,132,133]$

$$
p(T, \mu)=T \sum_{k=1}^{\infty} b_{k}(T)[g \phi(T ; m) \lambda]^{k}=T \sum_{k=1}^{\infty} b_{k}(T) z^{k} .
$$

Here $z \equiv g \phi(T ; m) \lambda$ is the absolute activity, which can be considered as the density of the ideal gas with Boltzmann statistics at a given $T$ - $\mu$ pair, and $b_{k}(T)$ are the cluster integrals, i.e. the coefficients of the Mayer's cluster expansion in fugacities (see, e.g., Chapter 10 in Ref. [132]). Function $\phi(T ; m)$ is expressed via the modified Bessel function $K_{2}$,

$$
\phi(T ; m)=\frac{m^{2} T}{2 \pi^{2}} K_{2}\left(\frac{m}{T}\right)
$$

where we assumed the relativistic dispersion relation $\varepsilon(k)=\sqrt{m^{2}+k^{2}}$.

In the Grand Canonical Ensemble where $T$ and $\mu$ are the thermodynamic variable, the pressure of the EV model is given in terms of the transcendental equation $p^{\mathrm{ev}}(T, \mu)=p^{\mathrm{id}}\left(T, \mu-v p^{\mathrm{ev}}\right)$. Cluster expansion of the $\mathrm{EV}$ model pressure around the ideal gas pressure $p^{\text {id }}(T, \mu)$ yields

$$
\begin{aligned}
p^{\mathrm{ev}}(T, \mu) & =p^{\mathrm{id}}\left(T, \mu-v p^{\mathrm{ev}}\right) \\
& =p^{\mathrm{id}}(T, \mu)-n^{\mathrm{id}}(T, \mu) v p^{\mathrm{ev}}(T, \mu)+\ldots \\
& =T g \phi(T ; m) \lambda-T v[g \phi(T ; m)]^{2} \lambda^{2}+O\left(\lambda^{3}\right),
\end{aligned}
$$


where $g$ is the internal degeneracy factor (for nucleons $g_{N}=4$ ). Note, that the effects of quantum statistics were neglected in the final line in Eq. (IV.10). Here and in further subsections only the behavior of the 2nd cluster or virial coefficients is analyzed, therefore, the expansion in Eq. (IV.10) is written only up to the 2nd order.

Comparison of Eqs. (IV.10) and (IV.8) yields

$$
b_{2}^{\mathrm{ev}}=-v=-a_{2}^{\mathrm{ev}} .
$$

Thus, the 2nd cluster integral is straightforwardly connected to the excluded volume parameter $v$.

\section{IV.2 Beth-Uhlenbeck approach}

\section{IV.2.1 Formalism}

Interactions in a thermodynamic quantum system can be described by both the virial (IV.3) and the cluster (IV.8) expansions. In the case of only elastic interactions which do not create bound states, the 2nd cluster integral can be expressed by the generalized BU formula $[134,135,136]^{(1)}$

$$
b_{2}(T)=[g \phi(T ; m)]^{-2} \frac{T}{2 \pi^{3}} \int_{2 m}^{\infty} d \varepsilon \varepsilon^{2} \mathrm{~K}_{2}(\varepsilon / T) \sum_{Q} g_{Q} \frac{d \delta_{Q}(\varepsilon)}{d \varepsilon} .
$$

Here the integration is carried over all values of the invariant mass $\varepsilon$ of two particle system in the center-of-mass frame. The summation is done over all relevant channels of all two-particle states which have a set of quantum numbers $Q$. The specific definition of $Q$ depends on a particular system studied (see below). $\delta_{Q}(\varepsilon)$ is the corresponding scattering phase shift for channel $Q$. In the equation (IV.12) the relativistic dispersion relation $\varepsilon(k)=\sqrt{m^{2}+k^{2}}$ between energy and momentum is assumed.

Furthermore, a system of interacting nucleons is considered. For nucleonnucleon scattering, the corresponding set of quantum numbers is $Q=(T, S, L, J)$ : isospin $T=0,1$; spin $S=0,1$; orbital momentum $L$; total angular momentum $J$, which takes the values $|L-S|<J<(L+S)$. The value of the orbital momentum

\footnotetext{
${ }^{(1)}$ The contribution of quantum statistics to $b_{2}(T)$ of ideal gas is found to be negligible for the applications considered in the present paper and is neglected for simplicity.
} 
$L$ determines the symmetry of the coordinate part of the two-nucleon wave function with respect to the exchange of the coordinates of two nucleons: the coordinate part is symmetric for even values of $L$, while it is antisymmetric for odd values of $L$. The total two-nucleon wave function is antisymmetric with respect to the exchange of their indices. Hence, $L$ is odd valued if the spin-isospin part is symmetric, and is even valued otherwise. For the nucleon system, the summation in (IV.12) is done over all possible $(T, S, L, J)$ values that are consistent with the above restrictions.

For the hard-sphere scattering potential (IV.6), the scattering phase-shifts can be calculated analytically by [137]

$$
\delta_{L}^{\mathrm{hc}}(\varepsilon)=\arctan \left[\frac{j_{L}\left(2 r_{c} q\right)}{n_{L}\left(2 r_{c} q\right)}\right] .
$$

Here $q \equiv q(\varepsilon)$ is the momentum of a particle in the c.m. frame, $j_{L}$ and $n_{L}$ are spherical Bessel functions. Assuming the relativistic dispersion relation, $q(\varepsilon)$ reads as $q(\varepsilon)=\frac{1}{2} \sqrt{\varepsilon^{2}-\left(2 m_{N}\right)^{2}}$. This yields the following expression for the 2 nd cluster integral of the nucleon system with a hard-core interaction

$$
\begin{aligned}
b_{2}^{N N}(T)=\left[g_{N} \phi\left(T ; m_{N}\right)\right]^{-2} \frac{T}{2 \pi^{3}} \int_{2 m_{N}}^{\infty} d \varepsilon \varepsilon^{2} \mathrm{~K}_{2}(\varepsilon / T) \\
\quad \times \sum_{T=0,1} \sum_{S=0,1} \sum_{L} \sum_{J=|L-S|}^{L+S}(2 T+1)(2 J+1) \frac{d \delta_{L}^{\mathrm{hc}}(\varepsilon)}{d \varepsilon} .
\end{aligned}
$$

After integrating by parts one obtains

$$
\begin{aligned}
b_{2}^{N N}(T)=[ & \left.g_{N} \phi\left(T ; m_{N}\right)\right]^{-2} \frac{1}{2 \pi^{3}} \int_{2 m_{N}}^{\infty} d \varepsilon \varepsilon^{2} \mathrm{~K}_{1}(\varepsilon / T) \\
& \times \sum_{T=0,1} \sum_{S=0,1} \sum_{L} \sum_{J=|L-S|}^{L+S}(2 T+1)(2 J+1) \delta_{L}^{\mathrm{hc}}(\varepsilon) .
\end{aligned}
$$

As follows from Eq. (IV.11), the BU approach with hard-core interaction (BU-HC) approach predicts a temperature dependence of the excluded volume parameter as $v_{N N}(T)=-b_{2}^{N N}(T)$, already on the level of the 2 nd order virial expansion.

All possible nucleon-nucleon combinations, i.e. proton-proton, proton-neutron, and neutron-neutron scatterings, contribute to the $b_{2}^{N N}$. These contributions to the 2nd cluster integral $b_{2}^{p p}$ can be calculated separately, e.g., for a pure proton system. The proton-proton contribution coincides with the $b_{2}^{n n}$ coefficient of the 
pure neutron system due to isospin symmetry. The specific value of the isospin quantum number is fixed $T=1$, and $b_{2}^{p p}=b_{2}^{n n}$ reads

$b_{2}^{p p}(T)=\left[\left(g_{N} / 2\right) \phi\left(T ; m_{N}\right)\right]^{-2} \frac{1}{2 \pi^{3}} \int_{2 m_{N}}^{\infty} d \varepsilon \varepsilon^{2} \mathrm{~K}_{1}(\varepsilon / T) \sum_{S=0,1} \sum_{L} \sum_{J=|L-S|}^{L+S}(2 J+1) \delta_{L}^{\mathrm{hc}}(\varepsilon)$.

It is also useful to estimate relativistic effects by considering the original non-relativistic BU formula [138],

$$
\begin{gathered}
b_{2}^{N N, \mathrm{nr}}(T)=\left[g_{N} \phi^{\mathrm{nr}}\left(T ; m_{N}\right)\right]^{-2} \frac{\left(2 m_{N}\right)^{2}}{2 \pi^{3}} \sqrt{\frac{\pi T}{4 m_{N}}} \exp \left(-\frac{2 m_{N}}{T}\right) \int_{0}^{\infty} d \varepsilon \exp (-\varepsilon / T) \\
\times \sum_{T=0,1} \sum_{S=0,1} \sum_{L} \sum_{J=|L-S|}^{L+S}(2 T+1)(2 J+1) \delta_{L}^{\mathrm{hc}}(\varepsilon),
\end{gathered}
$$

where

$$
\phi^{\mathrm{nr}}(T ; m)=\left(\frac{m T}{2 \pi}\right)^{3 / 2} \exp \left(-\frac{m}{T}\right)
$$

A comparison of the non-relativistic BU-HC result (IV.17) with the classical result (IV.7) provides an important cross check. At high temperatures, the quantum effects in the BU-HC model become unimportant, hence, the results of (IV.17) and (IV.7) coincide.

\section{IV.2.2 Calculation results}

Figure IV.1 depicts the temperature dependence of the nucleon-nucleon excluded volume parameter $v_{N N}$, calculated using Eq. (IV.15) for the nucleon hard-core radius of $r_{c}=0.3 \mathrm{fm}$. The temperature dependencies of the contribution NN systems are depicted as well: proton-proton eigenvolume $v_{p p}$, and of proton-neutron eigenvolume $v_{p n}=2 v_{N N}-v_{p p}$. The classical result (IV.7) is depicted by the solid horizontal line. In the numerical evaluation of Eq. (IV.15) the terms with $L>10$ are disregarded. It was numerically checked that the higher order terms give a negligible contribution to $b_{2}^{\mathrm{NN}}$ for temperatures up to $T=300 \mathrm{MeV}^{(2)}$.

\footnotetext{
${ }^{(2)}$ The figures presented consider rather high temperatures. These temperatures are presented to illustrate a connection between different model formulations. In reality, hadrons are not expected to be the dominant constituents of the strongly interacting matter at $T>200 \mathrm{MeV}$.
} 


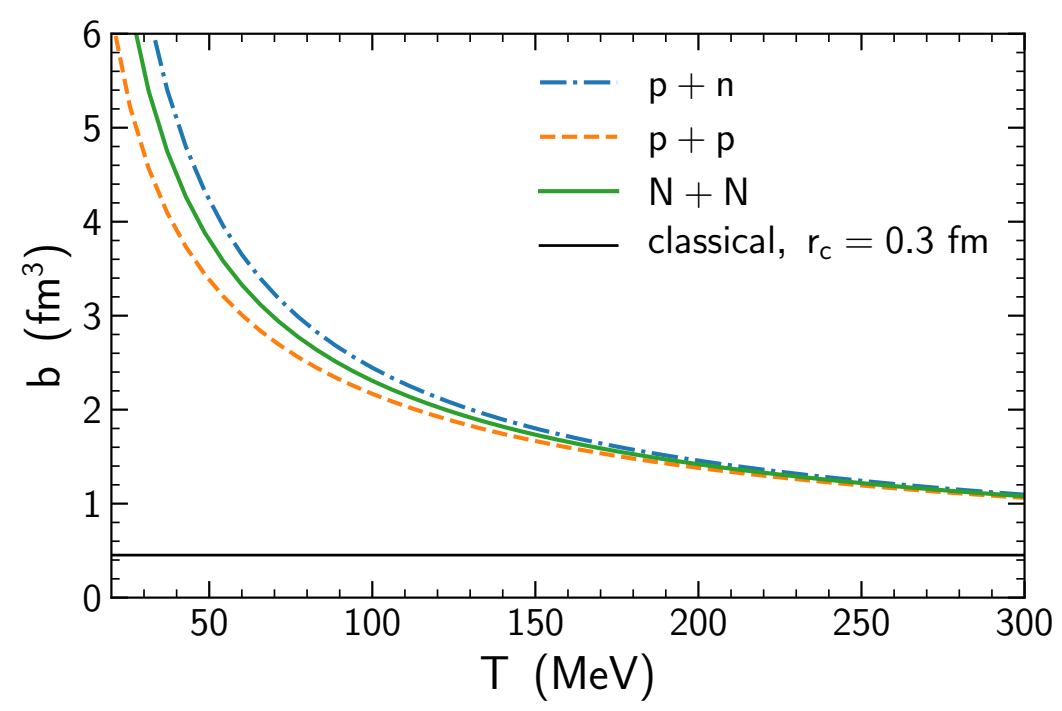

Figure IV.1: The temperature dependence of the nucleon-nucleon excluded volume parameter $v_{\mathrm{NN}}$ (solid green line), the proton-proton excluded volume parameter $v_{p p}$ (dashed yellow line), and the proton-neutron excluded volume parameter $v_{p n} \equiv 2 v_{\mathrm{NN}}-v_{p p}$ (dot-dashed blue line), as calculated within the relativistic BethUhlenbeck approach for a hard-core potential with the nucleon hard-core radius of $r_{c}=0.3 \mathrm{fm}$. The dashed horizontal line shows the prediction of the classical EV model (IV.7) with the same value of $r_{c}=0.3 \mathrm{fm}$.

The Figure IV.1 suggests that the classical EV model [Eq. (IV.10)] underestimates the value of the nucleon-nucleon excluded volume parameter by large factors of $3-4$, at temperatures $T=100-200 \mathrm{MeV}$. This temperature range is rather typical for the phenomenological applications of the EV model in the context of heavy-ion collisions and (Lattice) QCD equation of state. A strong increase of $v_{N N}$ at low temperatures correlates with an increase of the thermal wavelength $\lambda_{d B}$.

This result is quite remarkable: the hard-core radii of hadrons are often used as an input into the classical EV-HRG model to describe the repulsive interactions between hadrons at high densities (see e.g. Refs. [115, 139, 140, 22, 141, 142, $143,144,145,116,146,147])$. A value $r_{c}=0.3 \mathrm{fm}$ is sometimes taken based on the properties of microscopical nucleon-nucleon scatterings $[141,143]$. The large discrepancy between the classical EV model and the BU approach suggests that the former can only be considered as a simplified effective approach when used 
in hadronic physics applications. This means that the parameter $a_{2}^{\text {ev }}$ of the EV model should not be connected to the values of the hard-core radii via Eq. (IV.7). Note that similar concerns were presented before, based on BU calculations for spinless particles $[136,148]$. More accurate analyses shall also take into account interaction-channel dependent hard-core radii [136, 149].

The classical EV model result [Eq. (IV.10)] is only valid when both, quantum mechanical and relativistic effects, can be neglected. The non-relativistic BU$\mathrm{HC}$ formula (IV.17) is expected to coincide with the classical result (IV.10) at high temperatures. This was proven for spinless particles with hard-core interactions $[136,148]$. A numerical check for spin- $1 / 2$ nucleons is presented in Figure IV.2, the temperature dependence of the nucleon-nucleon excluded volume parameter $v_{N N}$, as calculated in the non-relativistic (solid blue line) and relativistic (dash-dotted red line) BU-HC approach, for $r_{c}=0.3 \mathrm{fm}$, is shown on a logarithmic temperature scale, in the range $T=10^{1}-10^{6} \mathrm{MeV}$. Note, in the present work, the difference between the relativistic and non-relativistic BU approaches appears only in the dispersion relation between energy and momentum. At very high temperatures, $T \sim 10^{5} \mathrm{MeV}$, the excluded volume parameter of the non-relativistic BU formula approaches the classical limit (dashed line) from above, as expected. These huge temperatures, however, can not be related to any practical applications since nucleons are expected to already melt into partons there.

The behavior of $v_{N N}(T)$ in the relativistic and in the non-relativistic BU-HC approaches are similar. The relativistic approach yields systematically smaller values of $v_{N N}(T)$. The limiting value of relativistic $v_{N N}(T)$ is slightly below the classical limit. Note that a relativistic formulation of the hard-core interaction problem is not fully consistent, since the concept of hard-core interactions is inconsistent with causality. However, at the temperature range of interest for hadronic physics applications is not affected that strongly by relativistic effects. Therefore, the treatment of the hard-core repulsion between nucleons within the relativistic BU-HC approach is considered satisfactory.

Scattering phase shifts can also be employed to study the non-equilibrium properties of interacting hadrons [150]. Hence, one can study in a similar fashion the differences between the classical and quantum mechanical hard-core repulsion mechanisms, such as the scattering cross section and transport coefficients. 


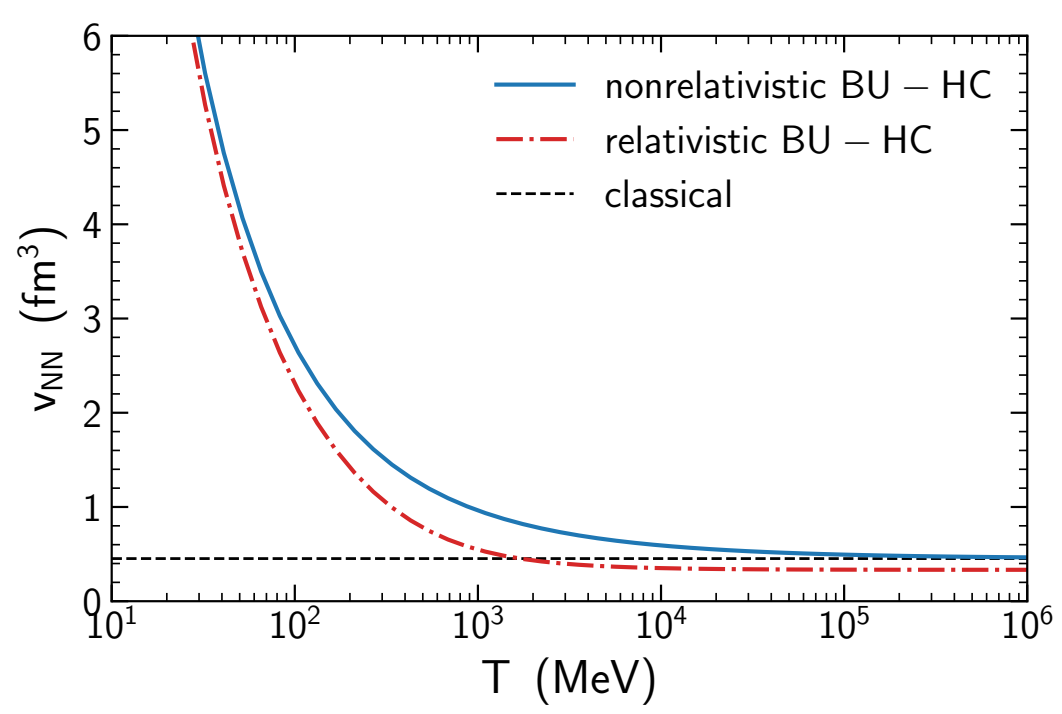

Figure IV.2: The temperature dependence of the nucleon-nucleon excluded volume parameter $v_{\mathrm{NN}}$ calculated using the non-relativistic (solid blue line) and relativistic (dash-dotted red line) dispersion relations in the Beth-Uhlenbeck approach for hard core interaction potential, shown on the logarithmic temperature scale. Nucleon hard-core radius of $r_{c}=0.3 \mathrm{fm}$ is assumed. The dashed horizontal line shows the prediction of the classical EV model (IV.7) with the same value of $r_{c}=0.3 \mathrm{fm}$.

\section{IV.2.3 Other estimates and the role of attraction}

The present approach can be compared to other estimates of the 2nd virial coefficient for nucleons. The 2 nd virial coefficient should not be only associated with a hard-core interaction potential and is not identified exclusively with an eigenvolume parameter. Therefore, we use the notation $a_{2}^{N N}$ instead of $v_{N N}$ for this comparison. The comparison illustrates the relevance of the hard-core repulsion for the thermodynamics of a nucleon gas.

For the hard-core repulsion, the empirical values of the nucleon hard-core radius $r_{c}$ are considered in the range $r_{c}=0.25-0.30 \mathrm{fm}$, as suggested by the analysis of $N N$-scattering phase shift data [151]. The corresponding BU result is depicted in Fig. IV.3 by the blue band. Decreasing $r_{c}$ from $0.3 \mathrm{fm}$ to $0.25 \mathrm{fm}$ results in about $30 \%$ decrease of $a_{2}^{N N}(T)$ at a given temperature.

The present BU-HC approach only accounts for the short range repulsive hard-core interactions in the second virial coefficient. However, at an intermediate 


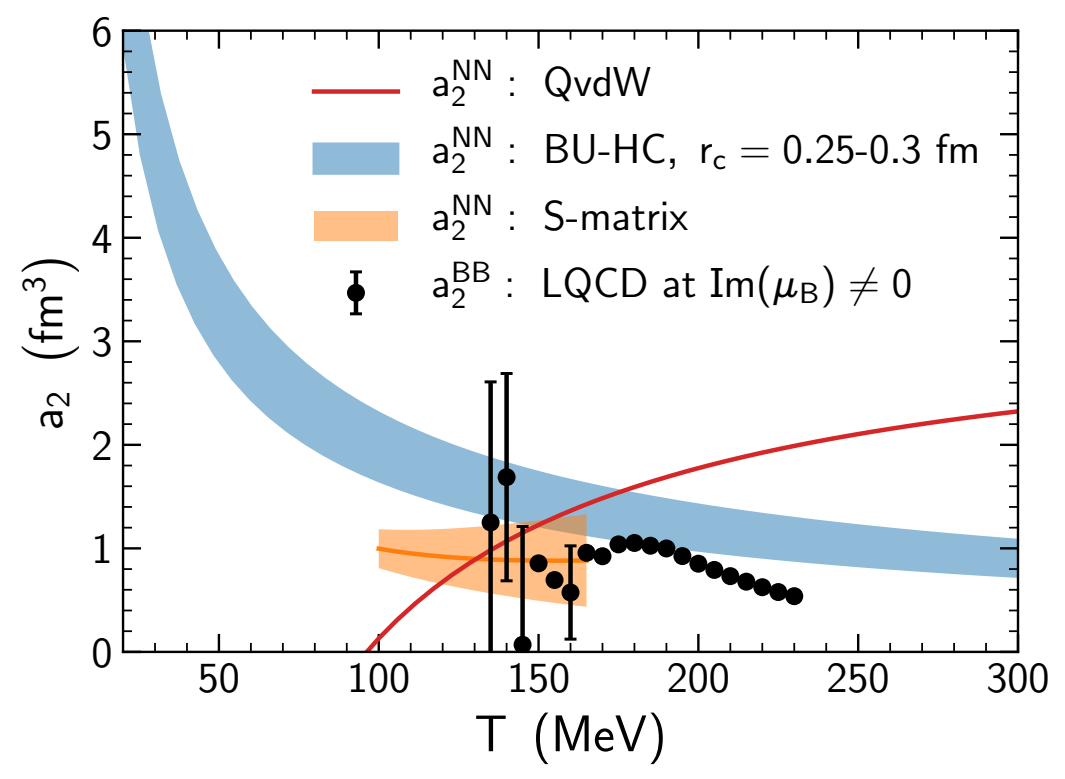

Figure IV.3: The temperature dependence of the second virial coefficient $a_{2}(T)$ of nucleon-nucleon interaction, calculated within different approaches. The calculations within the relativistic Beth-Uhlenbeck approach for the system of nucleons with a hard-core interaction are depicted by the blue band, which results from the variation of the nucleon hard-core radius in the range $0.25<r_{c}<0.30 \mathrm{fm}$. The calculations of Ref. [152] within the S-matrix formalism, employing the empirical phase shifts of $N N$-scattering, are depicted by the yellow line with a band. The red line depicts the second virial coefficient of nucleon-nucleon interaction in the quantum van der Waals model of nuclear matter [153]. Lattice QCD results for the 2nd virial coefficient of "baryon-baryon interaction" [118], obtained from simulations at an imaginary baryochemical potential, are depicted by black symbols with error bars.

distance range, the nucleon-nucleon interactions are also attractive. Attractive interactions give sizable negative contributions to $a_{2}^{N N}$. Especially at low temperatures, $T<20 \mathrm{MeV}$, calculations [154] based on empirical phase shift data do suggest that attractive interactions give the dominant contribution to $a_{2}^{N N}$. Thus, the large positive contribution of the hard-core repulsion at low temperatures, as seen in Figs. IV.1-IV.3, is compensated by a similarly large, but negative contribution from the attraction.

A simple model which takes into account both attractive and repulsive inter- 
actions between nucleons is the Quantum van der Waals (QvdW) model [153]. The QvdW model takes into account the effects of quantum Fermi statistics which are important for the description of the nuclear matter in the region of small temperatures and large baryon densities. In the QvdW the repulsive and attractive interactions between nucleons are characterized by the temperature independent $\mathrm{vdW}$ parameters $b$ and $a$, respectively. A fit to the nuclear ground state properties at $T=0$ yields values of $b=3.42 \mathrm{fm}^{3}$ and $a=329 \mathrm{MeV} \mathrm{fm}{ }^{3}$ for nucleons [153]. The second virial coefficient in this QvdW model is expressed as ${ }^{(3)}$ $a_{2}(T)=b-a / T$. The temperature dependence of $a_{2}^{N N}(T)$ in the QvdW model is depicted in Fig. IV.3, red line. $a_{2}^{N N}$ is negative at small temperatures, crosses zero at $T=a / b \simeq 96 \mathrm{MeV}$, and increases monotonically at larger temperatures. This sign change of $a_{2}^{N N}(T)$ is expected for any system of interacting particles with short-range repulsion and intermediate range attraction. At the same time, the continued increase of $a_{2}^{N N}(T)$ in the high temperature region in the QvdW model appears to be different from the results of the BU-HC formalism. This occurs because of the large temperature independent value of the excluded-volume parameter $b$ in the QvdW model. Assuming $b=16 \pi r_{c}^{3} / 3$ one finds $r_{c} \cong 0.59 \mathrm{fm}$. This is essentially larger than $r_{c}=0.2-0.3 \mathrm{fm}$ for the BU-HC results presented in Fig. IV.3.

Empirical details of nucleon-nucleon microscopical interactions can be considered in the second virial coefficient via the S-matrix approach. This will allow employing the empirically known phase shifts of $N N$-scattering. Recently it was done in Ref. [152] for intermediate temperature range $100<T<165 \mathrm{MeV}$. The result of these calculations is depicted by the yellow band in Fig. IV.3. The width of the band is a result of the uncertainties in the contributions of the inelastic $N N$ channels to $a_{2}^{N N}(T)$. The S-matrix result for $a_{2}$ of Ref. [152] is slightly suppressed as compared to our BU calculation. This is expected, the S-matrix calculation takes into account both the attraction and repulsion between the nucleons to $a_{2}^{N N}(T)$. The overestimation of $a_{2}^{N N}$ by the BU-HC calculation is apparent, as in by design only the repulsive hard-core interactions between nucleons are considered. The difference between the present calculation and the S-matrix calculation of Ref. [152] is reduced at higher temperatures: this reflects the fact that the short-range repulsive interactions dominate at higher temperatures.

To make the comparison complete, the imaginary- $\mu_{B}$ lattice QCD calculations

${ }^{(3)}$ Again, here the small ideal Fermi gas contribution to $a_{2}(T)$ is neglected. 
of the partial pressure of QCD in the baryon number $|B|=2$ sector [118] are also shown in Fig. IV.3 by black circles. A purely hadronic description, reasonable at moderate temperatures, yields partial pressure proportional to an "average" second virial coefficient $a_{2}^{B B}$ for baryon-baryon interactions. The lattice estimations for $a_{2}^{N N}$ at $T<160 \mathrm{MeV}$ provide rather large error bars. The lattice results lie below the results of the BU calculations.

The above analysis of Fig. IV.3 $r_{c}=0.25 \mathrm{fm}$ is a reasonable estimate for the BU-HC calculation for $a_{2}^{N N}$ in the crossover temperature region, $T \sim 150 \mathrm{MeV}$. The BU-HC approach overestimates $a_{2}^{N N}$ at smaller temperatures due to the missing attractive interactions. Therefore, modifications of the BU-HC approach are desirable for applications at these temperatures.

\section{IV.3 Estimating the hadron repulsion from the lattice QCD data}

The BU-HC formalism discussed above can come into use to model the repulsive interactions between baryons in the HRG model. Here the approach of Ref. [155] is followed, so an extension of the ideal HRG model with repulsive interactions only between pairs of baryons and between pairs of antibaryons is employed. Hence, the system consists of three independent subsystems: non-interacting mesons, interacting baryons, and interacting antibaryons. Thus, the total pressure can be split into three contributions $p=P_{M}+P_{B}+P_{\bar{B}}$. Additionally, it is assumed that at vanishing baryon density the 2 nd virial coefficient, $v_{B B}(T)$, which characterizes the baryon-baryon interactions, is the same for all (anti-)baryon pairs at a given temperature. The nucleon-nucleon values, $v_{N N}(T)$, are assumed for all baryon-baryon and antibaryon-antibaryon pairs, i.e. $v_{B B}(T) \equiv v_{N N}(T)$. This simplifying assumption of similar repulsive core for different baryon pairs is supported by lattice QCD simulations [156]. This model does not take into account the role of high thermal pressure on all hadron volumes $[157,158]$, hence, probably overestimates the repulsive effects at high temperatures.

The partial pressure of the baryonic and antibaryonic subsystems in the BU-HC approach is expressed as

$$
\begin{aligned}
& P_{B}^{\mathrm{BU}}\left(T, \mu_{B}\right)=T \phi_{B}(T) \lambda_{B}-T v_{B B}(T)\left[\phi_{B}(T) \lambda_{B}\right]^{2}, \\
& P_{\bar{B}}^{\mathrm{BU}}\left(T, \mu_{B}\right)=T \phi_{B}(T) \lambda_{B}^{-1}-T v_{B B}(T)\left[\phi_{B}(T) \lambda_{B}^{-1}\right]^{2},
\end{aligned}
$$


where $\lambda_{B}=\exp \left(\mu_{B} / T\right)$ and

$$
\phi_{B}(T)=\sum_{i \in B} \int d m \rho_{i}(m) \frac{d_{i} m^{2} T}{2 \pi^{2}} K_{2}\left(\frac{m}{T}\right)
$$

is the baryonic spectrum, $d_{i}$ is the degeneracy- and $\rho_{i}$ is a properly normalized mass- distributions for hadron type $i$, and where the sum goes over all baryons in the system. We include all baryon states, which are listed as "confirmed" in the Particle Data Tables [159]. The non-zero widths of the resonances are assumed to have Breit-Wigner shapes and are taken into account in the function $\rho_{i}$, following Refs. [160, 161].

The model given by Eq. (IV.19) is titled BU-HRG. The baryonic pressures (IV.19a) and (IV.19b) contain only quadratic interaction terms, which are proportional to the 2nd cluster integral. At large enough values of temperature and/or fugacity, the baryonic pressure becomes negative due to the negative sign of the quadratic term. Thus, this pure BU approach is expected to break down at some point, when the higher order terms of the cluster expansion become significant. in the scope of the previous results, it is instructive to consider the EV-HRG model with an effective temperature dependent excluded volume parameter. The partial pressure of baryons and of antibaryons in such a model reads ${ }^{(4)}$

$$
\begin{aligned}
& P_{B}^{\mathrm{ev}}\left(T, \mu_{B}\right)=T \phi_{B}(T) \lambda_{B} \exp \left(-\frac{v_{B B}(T) P_{B}^{\mathrm{ev}}\left(T, \mu_{B}\right)}{T}\right), \\
& P_{\bar{B}}^{\mathrm{ev}}\left(T, \mu_{B}\right)=T \phi_{B}(T) \lambda_{B}^{-1} \exp \left(-\frac{v_{B B}(T) P_{\bar{B}}^{\mathrm{ev}}\left(T, \mu_{B}\right)}{T}\right) .
\end{aligned}
$$

The pressure of the EV-HRG model suggested in (IV.21) is consistent with the BU approach (IV.19) up to the second order of the cluster expansion. However, the EV-HRG model also contains nonzero higher order coefficients in the cluster expansion. Hence, the large differences between the two models may indicate that the second order cluster expansion is not applicable any longer.

The temperature dependence of the baryon susceptibilities at $\mu_{B}=0$ of the $n$-th order $\chi_{n}^{B}$ is defined as

$$
\chi_{n}^{B}=\left.\frac{\partial^{n}\left(p / T^{4}\right)}{\partial\left(\mu_{B} / T\right)^{n}}\right|_{\mu_{B}=0} .
$$

These higher-order susceptibilities are important measures of how the systems respond to changes in the $\mu_{B} / T$ values and are especially sensitive to the various

\footnotetext{
(4) The Fermi statistics effects are small in the considered temperature region and at $\mu_{B}=0$.
} 

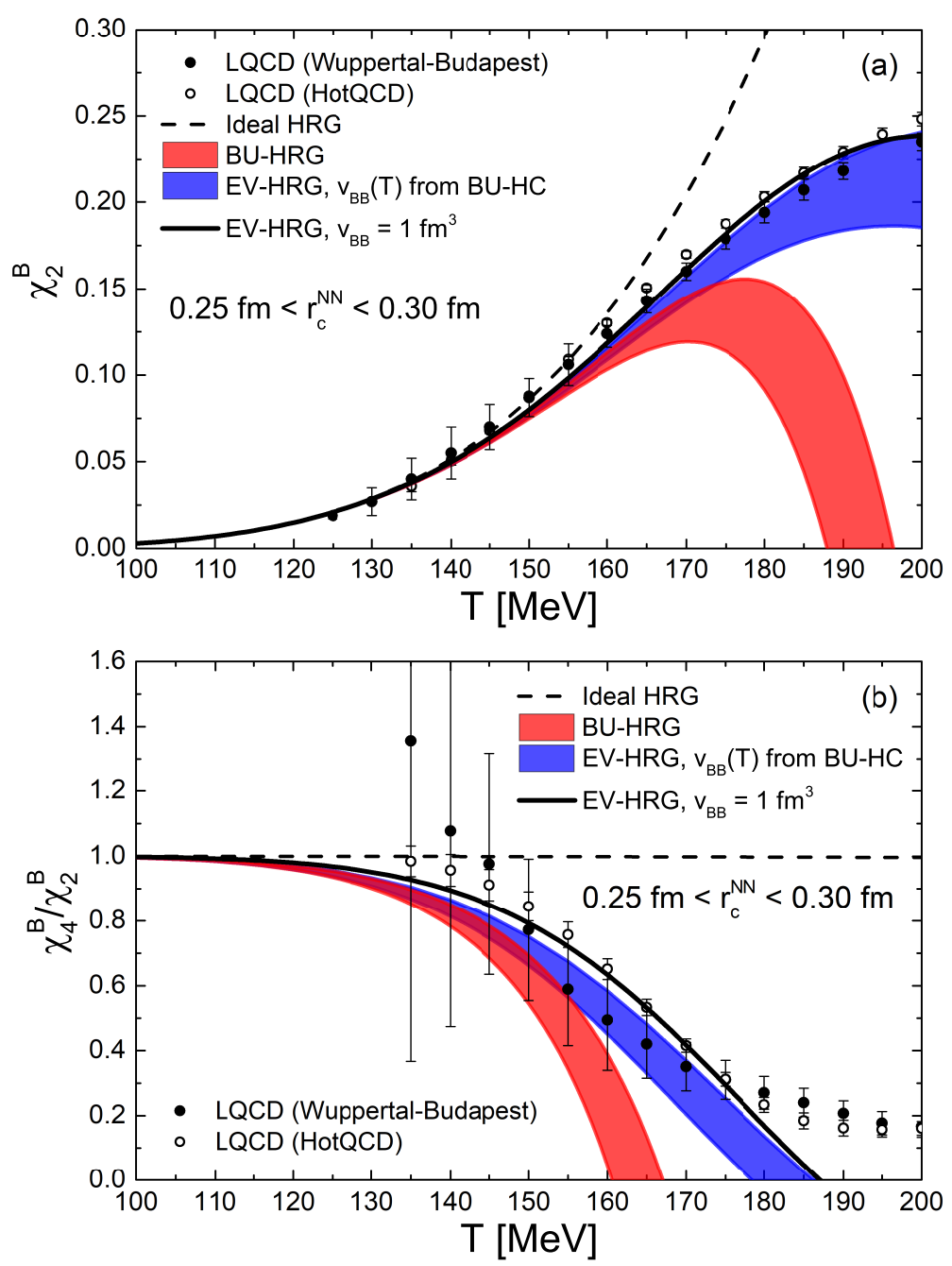

Figure IV.4: The temperature dependence of (a) $\chi_{2}^{B}$ and (b) $\chi_{4}^{B} / \chi_{2}^{B}$ net baryon number susceptibilities at $\mu_{B}=0$, as calculated within the I-HRG model (dashed black lines), the BU-HRG model (red bands), and the EV-HRG model (blue bands) with the temperature dependent baryon excluded volume parameter, using for all (anti)baryons the Beth-Uhlenbeck value for nucleons. The bands result from the variation of the nucleon hard-core radius in the range $r_{c} \simeq 0.25-0.3 \mathrm{fm}$. The lattice QCD results of the Wuppertal-Budapest $[162,163]$ and HotQCD [164, 165] collaborations are shown by the full and open symbols, respectively. Solid lines correspond to the EV-HRG model with $v_{B B}=1 \mathrm{fm}^{3}$.

baryon-baryon interactions. In Figure IV.3 the effects of the repulsive hard-core interactions between baryons are considered for these observables: the BU-HC 
calculations of $v_{N N}(T)$ for nucleons with $r_{c}=0.25-0.3 \mathrm{fm}$, are used for $v_{B B}(T)$ in Eqs. (IV.21a) and (IV.21b).

The resulting $\chi_{2}^{B}(T)$ and $\chi_{4}^{B}(T) / \chi_{2}^{B}(T)$ are depicted in Fig. IV.4. The red bands correspond to the BU-HRG model (IV.19a,IV.19b), the blue bands depict the EV-HRG model (IV.21a,IV.21b), and the ideal HRG model results are shown by the dashed lines. The lattice QCD results of the Wuppertal-Budapest $[162,163]$ and HotQCD [164, 165] collaborations are shown by the full and open symbols, respectively. At lower temperatures, $T \lesssim 110 \mathrm{MeV}$, the effect of the repulsive interactions on $\chi_{2}^{B}(T)$ and $\chi_{4}^{B}(T) / \chi_{2}^{B}(T)$ is negligible since the system pressure is low enough there. This is in spite of the strong increase of the excluded-volume parameter in the BU-HC approach at low temperatures. The effect is small because of an exponential decrease of the density of baryons, which renders the influence of baryonic interactions negligible at low temperatures and $\mu_{B}=0$. Repulsive baryon-baryon interactions suppress baryon susceptibilities at higher temperatures, compared to the ideal HRG result. At moderate temperatures, $T \lesssim 150 \mathrm{MeV}$ for $\chi_{2}^{B}$, and $T \lesssim 130 \mathrm{MeV}$ for $\chi_{4}^{B} / \chi_{2}^{B}$, this suppression is described nearly identically in BU-HRG and EV-HRG models. The total densities of baryons and of antibaryons at $\mu_{B}=0$ increase strongly as the temperature is increased. Higher terms of the cluster expansion become significant at higher temperatures. That can be noted already by the presence of large differences between the BUHRG and the EV-HRG models at $T \gtrsim 160 \mathrm{MeV}$. Figure IV.4 suggests that $\chi_{2}^{B}$ is negative at $T \gtrsim 190 \mathrm{MeV}$ in the BU-HRG model. By definition, $\chi_{2}^{B}$ characterizes the width of the fluctuations of the net baryon number. The negative values of $\chi_{2}^{B}$ in the BU-HRG model signal the breakdown of the second order virial expansion at high temperatures.

In contrast, the EV-HRG model predicts a reasonable behavior of the baryon number susceptibilities even at higher temperatures. The EV-HRG calculations with $v_{B B}(T)=v_{N N}(T)$ calculated within BU-HC approach for $r_{c}=0.25 \mathrm{fm}$ give an overall satisfactory description of the lattice data up to $T \simeq 175-180 \mathrm{MeV}$. The deviations of the ideal HRG model from lattice QCD data for the baryon susceptibilities in the vicinity and even above the pseudocritical temperature can be understood in terms of the repulsive baryonic interactions. This conclusion was reported previously in Refs. [155, 166, 152].

Calculations of $\chi_{2}^{B}$ and $\chi_{4}^{B} / \chi_{2}^{B}$ within the EV-HRG model with a constant temperature independent value $v_{N N}=1 \mathrm{fm}^{3}$, motivated by the $a_{2}^{N N}$ estimates 

58

in Fig. IV.3, are depicted in Fig. IV.4 (solid lines). This further improvement of the description of the lattice data in the crossover region by the EV-HRG model with $v_{N N}=1 \mathrm{fm}^{3}$ provides effectively a good approximation of the quantum description of baryon-baryon interactions in the crossover temperature region. Thus, this model can be used for the interpretation of the lattice QCD data; the model is also quite reasonable for the thermal analysis of baryon-related observables in heavy-ion collision experiments. Note that the value $v_{N N}=1 \mathrm{fm}^{3}$ was also suggested in the recent analysis of the lattice QCD data at imaginary baryochemical potential [118]. 


\section{A unified approach for QCD matter: Chiral Mean Field model}

In the previous chapters, the aspects of confined QCD matter were discussed with a main focus on the post freeze-out stages of heavy ion collisions. However, at high energy densities QCD matter appears in a deconfined state where free quarks are the dominant degrees of freedom and hadrons are suppressed. This chapter presents a unified approach for the QCD matter, the CMF model. The CMF model includes many aspects of QCD phenomenology, together with the HRG description and EV interactions discussed before, and allows for a self-consistent description of the hadron-quark transition, thus being applicable for the whole QCD phase diagram. Here, the CMF model is described in detail with all underlying equations and degrees of freedom. The quark sector of the CMF model is tuned to describe the $\mu_{B}=0$ thermodynamics data of lattice QCD. The resulting lines of constant physical variables as well as the baryon number susceptibilities are studied in some detail in the temperature/chemical potential plane. The CMF model predicts three consecutive transitions, the nuclear first-order liquid-vapor phase transition, chiral symmetry restoration, and the cross-over transition to a quark matter phase. All three phenomena are crossover for most of the $T-\mu_{B}$-plane. The deviations from the free ideal hadron gas baseline at $\mu_{B}=0$ and $T \approx 100-200 \mathrm{MeV}$ can be attributed to remnants of the liquid-vapor first order phase transition in nuclear matter. The chiral crossing transition determines the baryon fluctuations at much 
higher $\mu_{B} \approx 1.5 \mathrm{GeV}$. At high baryon densities, $\mu_{B} \approx 2.4 \mathrm{GeV}$, the behavior of fluctuations is controlled by a cross-over to quark matter. The CMF model is applied to model stable neutron stars by solving the Tolman-Oppenheimer-Volkoff equation. The CMF predictions for the NSs masses, and radii are found to be in agreement with modern astrophysical constraints. The calculated values of NSs tidal deformabilities agree well with the values obtained from the analysis of gravitational waves emitted from binary neutron star mergers.

This chapter is based on the research performed by the author of this thesis and published in Refs.[2, 4, 5, 6, 8].

\section{V.1 Chiral Mean Field model}

The Chiral SU(3)-flavor parity-doublet Polyakov-loop quark-hadron mean-field model (or the CMF model) describes the thermodynamics of strongly interacting matter in different regimes. The CMF model allows to calculate the equation of state (EOS) of QCD matter at a wide range of temperatures and densities, as well as the transition from the hadronic to free quark regime. It incorporates major concepts of QCD phenomenology: chiral interactions in the baryon octet [167], the full PDG hadron list [93], excluded volume repulsive interactions among all hadrons [128, 168], baryon parity doubling [169], and quarks coupled to an effective Polyakov loop Potential (similar to the Polyakov Nambu Jona-Lasinio model [170]).

The main component of the CMF model is the three flavor chiral Lagrangian for strange hadronic matter first introduced in [167]. The Lagrangian $\mathcal{L}_{S U(3)_{f}}$ consists of the following parts:

$$
\mathcal{L}_{S U(3)_{f}}=\mathcal{L}_{\mathcal{B}}+U_{\text {sc }}+U_{\text {vec }}
$$

here $\mathcal{L}_{\mathcal{B}}$ describes the scalar and vector mean field interactions between the ground state octet baryons and their parity partners:

$$
\begin{aligned}
\mathcal{L}_{\mathcal{B}} & =\sum_{b}\left(\bar{B}_{b} i \not \partial B_{b}\right)+\sum_{b}\left(\bar{B}_{b} m_{b}^{*} B_{b}\right) \\
& +\sum_{b}\left(\bar{B}_{b} \gamma_{\mu}\left(g_{\omega b} \omega^{\mu}+g_{\rho b} \rho^{\mu}+g_{\phi b} \phi^{\mu}\right) B_{b}\right),
\end{aligned}
$$

where the index $b$ runs through all ground-state baryons, $p, n, \Lambda, \Sigma^{+, 0,-}, \Xi^{0,-}$, and their respective parity partners, $N(1535)^{+, 0}, \Lambda(1405), \Sigma(1750)^{+, 0,-}, \Xi(1950)^{0,-}$. 
$U_{\text {sc }}$ describes the potential of the scalar $\sigma$ and $\zeta$ fields, and $U_{\text {vec }}$ is the potential of the vector $\omega, \rho$, and $\phi$ fields.

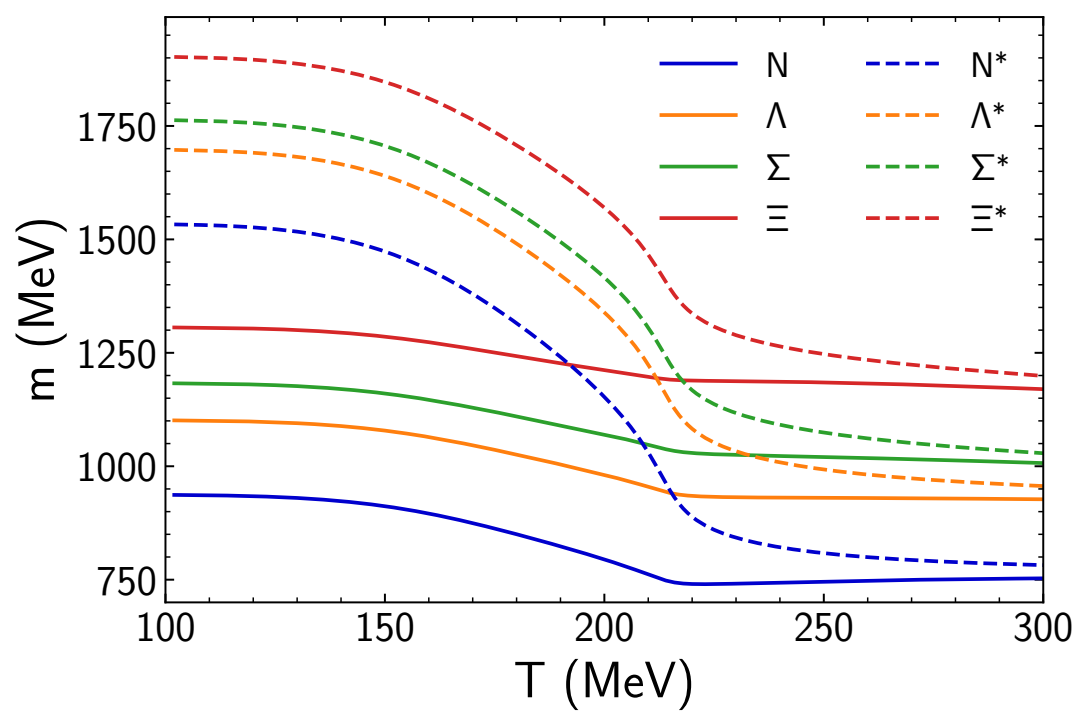

Figure V.1: Masses of baryon octet and their parity partners as functions of $T$ at $\mu_{B}=0$, note smooth appearance of mass degeneracy at high $T$ between ground state baryons and their respective parity partners.

The effective masses of the ground state octet baryons and their parity partners (assuming isospin symmetry) read [171]:

$$
\begin{aligned}
m_{b \pm}^{*} & =\sqrt{\left[\left(g_{\sigma b}^{(1)} \sigma+g_{\zeta b}^{(1)} \zeta\right)^{2}+\left(m_{0}+n_{s} m_{s}\right)^{2}\right]} \\
& \pm g_{\sigma b}^{(2)} \sigma
\end{aligned}
$$

where the various coupling constants $g_{* b}^{(*)}$ are determined by vacuum masses and by nuclear matter properties, the $\mathrm{SU}(3)$ breaking mass term that generates an explicit mass corresponding to the strangeness $n_{s}$ of the baryon is included. This approach describes parity doubling in the baryon octet implying a mass splitting between the baryon parity partners which is assumed to be generated by the scalar mesonic fields $\sigma$ and $\zeta[169,172,48,173]$.

The chiral field dynamics are determined self consistently by the scalar meson interaction potentials, driving the spontaneous breaking of the chiral symmetry:

$$
\begin{aligned}
U_{\mathrm{sc}} & =V_{0}-\frac{1}{2} k_{0} I_{2}+k_{1} I_{2}^{2}-k_{2} I_{4}+k_{6} I_{6} \\
& +k_{4} \ln \frac{\sigma^{2} \zeta}{\sigma_{0}^{2} \zeta_{0}}+U_{\mathrm{sb}},
\end{aligned}
$$


with

$$
\begin{aligned}
I_{2}=\left(\sigma^{2}+\zeta^{2}\right) & , \quad I_{4}=-\left(\sigma^{4} / 2+\zeta^{4}\right), \\
I_{6} & =\left(\sigma^{6}+4 \zeta^{6}\right)
\end{aligned}
$$

where $V_{0}$ is fixed by demanding that the pressure vanishes in the vacuum. In addition, an explicit symmetry breaking term is introduced in the scalar potential:

$$
U_{\mathrm{sb}}=m_{\pi}^{2} f_{\pi} \sigma+\left(\sqrt{2} m_{k}^{2} f_{k}-\frac{1}{\sqrt{2}} m_{\pi}^{2} f_{\pi}\right) \zeta
$$

The mean field vector repulsion is mediated by the fields: $\omega$ for repulsion at finite baryon densities, the $\rho$ for repulsion at finite isospin densities, and the $\phi$ for repulsion when finite strangeness density is generated. The vector fields depend on the respective conserved charge densities and are controlled by the potential $U_{\text {vec }}$

$$
\begin{aligned}
U_{\mathrm{vec}} & =-\frac{1}{2}\left(m_{\omega}^{2} \omega^{2}+m_{\rho}^{2} \rho^{2}+m_{\phi}^{2} \phi^{2}\right) \\
& -g_{4}\left(\omega^{4}+6 \beta_{2} \omega^{2} \rho^{2}+\rho^{4}+\frac{1}{2} \phi^{4}\left(\frac{Z_{\phi}}{Z_{\omega}}\right)^{2}\right. \\
& \left.+3\left(\rho^{2}+\omega^{2}\right)\left(\frac{Z_{\phi}}{Z_{\omega}}\right) \phi^{2}\right)
\end{aligned}
$$

Finally the remaining mesonic and non-interacting hadronic degrees of freedom are included in a form of Hadron Resonance Gas as a thermal heat bath according to their vacuum masses.

Altogether, the baryonic interactions allow for a reasonable description of nuclear matter properties. The coupling constants of the hadronic sector and the parameters of the effective potential for these fields (see [171] for details) are chosen such that the properties of nuclear matter are reproduced: ground state density $n_{0} \approx 0.16 \mathrm{fm}^{-3}$, the binding energy per nucleon is $E_{0} / B \approx-15.2 \mathrm{MeV}$, asymmetry energy $S_{0} \approx 31.9 \mathrm{MeV}$, and compressibility $K_{0} \approx 267 \mathrm{MeV}$. All fixed parameters and coupling constants used in the CMF model are summarized in table V.1.

The quark degrees of freedom are introduced as in the Polyakov-loop-extended Nambu Jona-Lasinio (PNJL) model [170], where their thermal contribution is directly coupled to the Polyakov Loop order parameter $\Phi[5]$, the quark thermal 
CHAPTER V. A UNIFIED APPROACH FOR QCD MATTER: CHIRAL

\begin{tabular}{cc||cc||cc}
\hline \hline$m_{\pi}$ & $138 \mathrm{MeV}$ & $g_{q \sigma}$ & -1 & $g_{\rho p, n}$ & \pm 4.55 \\
\hline$m_{K}$ & $498 \mathrm{MeV}$ & $g_{s \zeta}$ & -1 & $g_{\rho \Lambda}$ & 0 \\
\hline$m_{\omega}$ & $783 \mathrm{MeV}$ & $g_{\sigma N}^{(1)}$ & -9.45 & $g_{\rho \Sigma^{ \pm}}$ & \pm 3.63 \\
\hline$m_{\rho}$ & $761 \mathrm{MeV}$ & $g_{\sigma \Lambda}^{(1)}$ & -7.62 & $g_{\rho \Xi^{ \pm}}$ & \pm 1.816 \\
\hline$m_{\phi}$ & $1019 \mathrm{MeV}$ & $g_{\sigma \Sigma}^{(1)}$ & -5.83 & $g_{\phi N}$ & 0 \\
\hline$m_{0 q}$ & $253 \mathrm{MeV}$ & $g_{\sigma \Xi}^{(1)}$ & -4.89 & $g_{\phi \Lambda}$ & -3.34 \\
\hline$\delta m_{q}$ & $56 \mathrm{MeV}$ & $g_{\sigma B}^{(2)}$ & 3.21 & $g_{\phi \Sigma}$ & -3.34 \\
\hline$m_{s}$ & $130 \mathrm{MeV}$ & $g_{\zeta N}^{(1)}$ & -0.899 & $g_{\phi \Xi}$ & -6.69 \\
\hline$f_{\pi}$ & $93 \mathrm{MeV}$ & $g_{\zeta \Lambda}^{(1)}$ & -3.49 & $k_{0}$ & $242^{2} \mathrm{MeV}^{2}$ \\
\hline$f_{K}$ & $122 \mathrm{MeV}$ & $g_{\zeta \Sigma}^{(1)}$ & -6.02 & $k_{1}$ & 4.818 \\
\hline$m_{0}$ & $759 \mathrm{MeV}$ & $g_{\zeta \Xi}^{(1)}$ & -7.35 & $k_{2}$ & -23.3 \\
\hline$T_{0}$ & $180 \mathrm{MeV}$ & $g_{\zeta B}^{(2)}$ & 0 & $k_{4}$ & $76^{4} \mathrm{MeV}^{4}$ \\
\hline$a_{0}$ & 3.51 & $g_{\omega N}$ & 5.45 & $k_{6}$ & $10^{-4} \mathrm{MeV}^{-2}$ \\
\hline$a_{1}$ & -11.67 & $g_{\omega \Lambda}$ & 6 & $\beta_{2}$ & 1500 \\
\hline$a_{2}$ & 9.33 & $g_{\omega \Sigma}$ & 8.175 & $Z_{\phi}$ & 2.239 \\
\hline$b_{3}$ & -0.53 & $g_{\omega \Xi}$ & 4.905 & $Z_{\omega}$ & 1.322 \\
\hline \hline
\end{tabular}

Table V.1: List of default parameters and coupling constants of the CMF model.

contribution reads as:

$$
\begin{aligned}
\Omega_{\mathrm{q}}= & -V T \sum_{q_{i} \in Q} \frac{d_{q_{i}}}{(2 \pi)^{3}} \int d^{3} k \frac{1}{N_{c}} \ln \left(1+3 \Phi e^{-\left(E_{q_{i}}^{*}-\mu_{q_{i}}^{*}\right) / T}\right. \\
& \left.+3 \bar{\Phi} e^{-2\left(E_{q_{i}}^{*}-\mu_{q_{i}}^{*}\right) / T}+e^{-3\left(E_{q_{i}}^{*}-\mu_{q_{i}}^{*}\right) / T}\right)
\end{aligned}
$$

where the index $q_{i}$ runs through $u, d, s$ flavors. The anti-quark contribution can be obtained by replacing $\mu_{q_{i}}^{*} \rightarrow-\mu_{q_{i}}^{*}$, and $\Phi \leftrightarrow \bar{\Phi}$. The Polyakov-loop order parameter $\Phi$ effectively describes the gluon contribution to the thermodynamic potential and is controlled by the temperature-dependent potential [5]:

$$
\begin{aligned}
U_{\mathrm{Pol}}(\Phi, \bar{\Phi}, T) & =-\frac{1}{2} a(T) \Phi \bar{\Phi} \\
+b(T) \ln [1-6 \Phi \bar{\Phi} & \left.+4\left(\Phi^{3}+\bar{\Phi}^{3}\right)-3(\Phi \bar{\Phi})^{2}\right] \\
a(T) & =a_{0} T^{4}+a_{1} T_{0} T^{3}+a_{2} T_{0}^{2} T^{2} \\
b(T) & =b_{3} T_{0}^{4}
\end{aligned}
$$

The dynamical quark masses $m_{q}^{*}$ of the light and strange quarks are also 
determined by the $\sigma$ - and $\zeta$ - fields, with the exception of a fixed mass term $m_{0 q}$, which can be understood as the contribution of the gluon condensate to the quark mass:

$$
\begin{gathered}
m_{u, d}^{*}=-g_{u, d \sigma} \sigma+\delta m_{u}, d+m_{0 u, d} \\
m_{s}^{*}=-g_{s \zeta} \zeta+\delta m_{s}+m_{0 q} .
\end{gathered}
$$

The full grand canonical potential of the CMF model can be expressed as follows:

$$
\Omega=\Omega_{\mathrm{q}}+\Omega_{\overline{\mathrm{q}}}+\Omega_{\mathrm{h}}+\Omega_{\overline{\mathrm{h}}}-\left(U_{\mathrm{sc}}+U_{\mathrm{vec}}+U_{\mathrm{Pol}}\right)
$$

$\Omega_{\mathrm{h}}$ and $\Omega_{\overline{\mathrm{h}}}$ are the contributions from the hadrons which are the octet and the parity partners according to $\mathcal{L}_{\mathcal{B}}$ and the rest of the hadron list is incorporated in a form of a hadron resonance gas. $U_{\mathrm{sc}}$ is the mean field interaction potential of the scalar mean fields $\sigma$ and $\zeta$, and $U_{\text {vec }}$ of the repulsive vector mean fields $\omega$, $\rho$, and $\phi . U_{\text {Pol }}$ describe an effective gluon potential contribution as a part of the PNJL description.

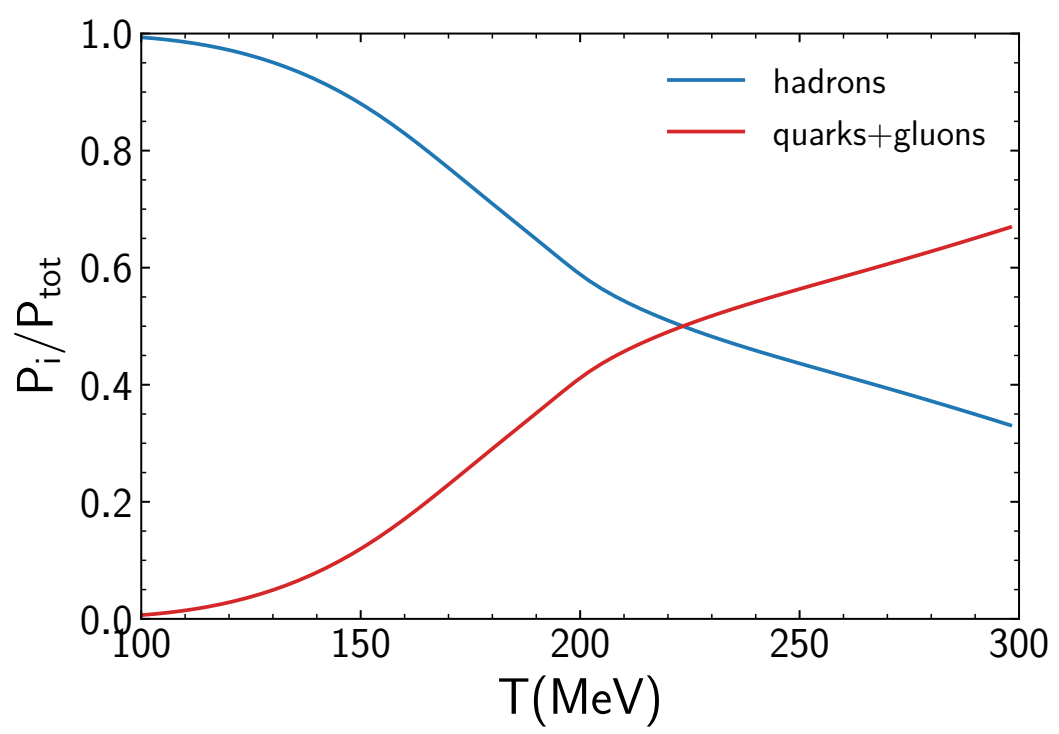

Figure V.2: Contribution to the total pressure separately from quarks+gluons and from hadrons as functions of temperature $T$ at $\mu_{B}=0$. The suppression of quarks at low temperatures is provided due to the values of Polyakov loop parameter $\Phi$ and $\bar{\Phi}$, hadrons at high $T$ are suppressed by excluded volume interactions. 
The transition between the quark and hadronic degrees of freedom is controlled by two mechanisms ${ }^{(1)}$ :

1. As the Polyakov loop order parameter becomes finite, free quarks can appear.

2. Hadrons are suppressed in the deconfined phase due to the excluded volume interactions.

The suppression of hadrons at high energy densities is maintained by their excludedvolume hard core interactions $[128,171]$. Due to the assumption of finite size, the hadrons are attributed an explicit volume term. This volume term then introduces an effective chemical potential $\mu_{j}^{\text {eff }}$ :

$$
\mu_{j}^{\mathrm{eff}}=\mu_{j}^{*}-v_{j} P
$$

for each hadronic particle specie $j$. Here, $P$ is the total pressure of the system and the $v_{j}$ are the EV parameters for the different particle species. The quarks in the CMF model are always assumed to be point-like.

As soon as quarks contribute to the pressure $P$, they reduce the hadronic density $\rho_{i}$ by lowering their chemical potential:

$$
\rho_{i}=\frac{\rho_{i}^{\mathrm{id}}\left(T, \mu_{i}^{*}-v_{i} P\right)}{1+\sum_{j \in \mathrm{HRG}} v_{j} \rho_{j}^{\mathrm{id}}\left(T, \mu_{j}^{*}-v_{j} P\right)},
$$

where $i$ refers to all possible contributions from baryons, mesons as well as quarks.

In its default version, the CMF model predicts two first order phase transitions for isospin symmetric matter. The nuclear liquid-vapor phase transition mimics the transition from dilute gas of nucleons to the dense nuclear matter, this transition is located at $\mu_{B} \approx m_{N}$ with critical temperature $T_{\mathrm{CP}} \approx 17 \mathrm{MeV}$. At higher densities, the CMF model exhibits a first order phase transition due to the chiral symmetry restoration among baryon parity partners $[171,175]$ with rather low critical temperature $T_{\mathrm{CP}} \approx 17 \mathrm{MeV}$. The transition occurs due to the rapid drop in the chiral condensates $\sigma$ and $\zeta$ so the mass gap between parity partners is reduced.

\footnotetext{
${ }^{(1)}$ Note in an earlier version of the CMF model which does not include the chiral partners of the baryons, the deconfinement phase transition is moderated by an additional $\Phi$ term in the effective mass of the fermions [174]. Here the point-like hadrons are suppressed by an explicitly $\mu_{B}$ dependent term in the Polyakov Loop potential.
} 


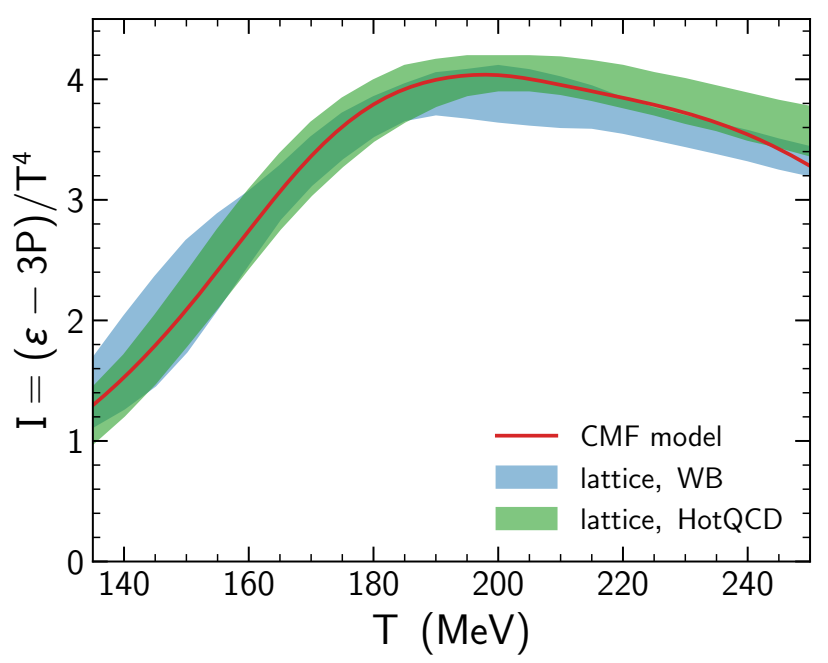

Figure V.3: Trace anomaly $I$ at $\mu_{B}=0$ as a function of temperature $T$. Comparison between model predictions and LQCD results [177, 24].

The CMF model can be applied to study neutron stars without changing its parameters. In this case, electric charge neutrality and $\beta$-equilibrium are imposed, so the conditions of the neutron star interior are fulfilled. To model the NS crust, which presumably consists of mostly neutron-rich nuclei and clusters in equilibrium, additional input is needed. That is done by matching the classical crust-EOS [176] to the CMF-EOS at $n_{B} \approx 0.05 \mathrm{fm}^{-3}$, such that below this density the matter is described by the crust EOS.

\section{V.2 Constraining the CMF model to the lat- tice data}

To introduce the constraints on the CMF model parameters from lattice QCD at high temperature and zero net baryon density the QCD trace anomaly I, "the interaction measure", can be used as a reference:

$$
\frac{I}{T^{4}}=\frac{\varepsilon-3 P}{T^{4}} .
$$

Trace anomaly $I$ is assumed to depict the appearance of quark degrees of freedom. $I$ effectively reflects the change of the number of degrees of freedom with an increase of temperature [178]. The hadronic models are capable of reproducing 
lattice data on $I$ for $T \lesssim 150 \mathrm{MeV}$ [171]. In this region, $I$ reflects the increase of the number of degrees of freedom due to the excitation of heavier resonances. However, the subsequent peak in $I$ and the following decrease is attributed to the appearance of quarks and gluons and so to the reduction of degrees of freedom. With this assumption, the free parameters of the present model, namely, parameters of the quark sector, are tuned to reproduce the lattice data for the trace anomaly.

The LQCD trace anomaly permits to calculate all observable thermodynamic quantities. An analysis of other Lattice data (chiral susceptibility) seem to show that for chirally related observables there is a crossover transition with a pseudo-critical temperature at $T \approx 156 \mathrm{MeV}$. The analysis of this data by a phenomenological model suggests a half-hadron, half-quark composition in that region [179].

The parameters of the CMF model's quark sector needed to reproduce the trace anomaly data from LQCD are found by a least mean squares fitting procedure for the parameters of the Polyakov loop potential $U_{\mathrm{Pol}}(\Phi, \bar{\Phi}, T)$ and for the coupling constants $g_{q \sigma}$ and $g_{s \zeta}$ of the quarks to the chiral condensates $\sigma$ and $\zeta$, respectively. All in all this fixes 5 model parameters, $T_{0}, a_{1}, a_{2}, b_{3}, g_{q \sigma}=g_{s \zeta}$ (we set $g_{q \sigma}$ and $g_{s \zeta}$ to the same value). The quark parameter fitting is performed through a scan over the parameter space on a $8 \times 6 \times 7 \times 6 \times 6$ sized grid, minimizing the root mean square deviation of the CMF model data on $I / T^{4}$ from the computed on the lattice results. The resulting parameter values are presented in Table V.2. A comparison of the CMF model to the lattice data is shown in Fig. V.3.

\begin{tabular}{ccccc}
$T_{0}(\mathrm{MeV})$ & $a_{1}$ & $a_{2}$ & $b_{3}$ & $g_{q \sigma}=g_{s \zeta}$ \\
\hline \hline 180.0 & -11.67 & 9.33 & -0.53 & -1.0
\end{tabular}

Table V.2: Best fit values of parameters extracted from a scan over the parameter space.

The values in Table V.2 suggest couplings $g_{q \sigma}, g_{s \zeta}$ of quarks to the chiral fields of about $1 / 3$ of the baryons, which one may expect from the additive quark model. Larger values of quark couplings would significantly influence the size of the peak in interaction measure $I / T^{4}$, as studied in Ref. [180]. In the case of large values of $g_{q \sigma}, g_{s \zeta}$ the strong interplay between the chiral symmetry restoration and the deconfinement transition would result in too large values of the interaction measure. The large peaks in the baryon number susceptibilities are in contrast to the lattice data. 


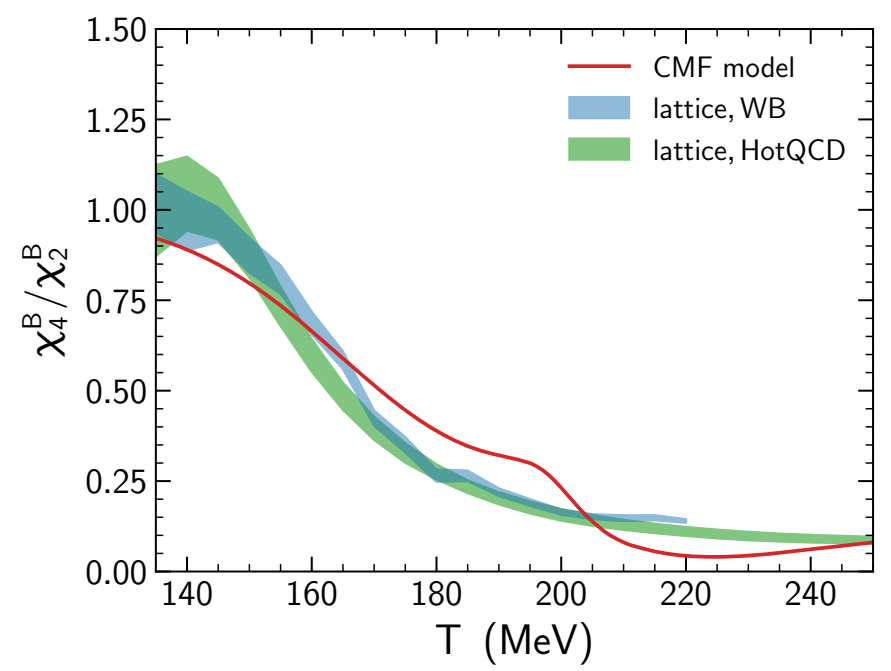

Figure V.4: The temperature dependence of net baryon number susceptibility ratio $\chi_{4}^{B} / \chi_{2}^{B}$ is shown as a function of temperature $T$. The red line depicts calculations within the CMF model, blue and green color bands are the results of the lattice QCD calculations from the Wuppertal-Budapest and the HotQCD collaborations, respectively [162, 163, 164, 165].

Higher order baryon number susceptibilities $\chi_{n}^{B}$ that are an LQCD measure of the particle number fluctuations

$$
\chi_{n}^{B}=\frac{\partial^{n}\left(P / T^{4}\right)}{\left(\partial \mu_{B} / T\right)^{n}},
$$

as well as the curvatures of various lines of constant physical quantities are also interesting in the scope of LQCD data.

The behavior of the $\chi_{4}^{B} / \chi_{2}^{B}$ at $\mu_{B}=0$ is presented in this section, the study for finite values of $\mu_{B}$ is presented in Sec. V.3. A comparison of the CMF model with the available LQCD data for the $\chi_{4}^{B} / \chi_{2}^{B}$ is shown in Fig. V.4, indicating a fair agreement of the CMF model with the lattice data.

Lattice QCD studies often explore regions of finite $\mu_{B}$ by using the Taylor series expansion. The Taylor expansion in a series of $T$ and $\mu_{B}$ up to $\mathcal{O}\left(\mu_{B}^{4}\right)$ was used in [164] to calculate "lines of constant physics": i.e. lines in the $T-\mu_{B}$ plane where certain thermodynamic quantities like pressure, energy density, and entropy density $P, \varepsilon, s$, are constant. The coefficients $\kappa_{2}^{f}$ and $\kappa_{4}^{f}(f \equiv P, \varepsilon, s)$ represent 
these contour lines in the $T-\mu_{B}$ plane using the following parametrization [164]:

$$
T_{f}\left(\mu_{B}\right)=T_{0}\left(1-\kappa_{2}^{f}\left(\frac{\mu_{B}}{T_{0}}\right)^{2}-\kappa_{4}^{f}\left(\frac{\mu_{B}}{T_{0}}\right)^{4}\right) .
$$

Here the coefficients $\kappa_{2}^{f}$ and $\kappa_{4}^{f}$ are calculated from Eqs. (V.17) and (V.19), see Ref. [164] for details:

$$
\kappa_{4}^{f}=\frac{\left.\frac{1}{2}\left(\kappa_{2}^{f}\right)^{2} T_{0}^{2} \frac{\partial^{2} f_{0}(T)}{\partial T^{2}}\right|_{\left(T_{0}, 0\right)} ^{f}=\frac{1}{T_{0}} \frac{f_{2}\left(T_{0}\right)}{\left.\frac{\partial f_{0}(T)}{\partial T}\right|_{\left(T_{0}, 0\right)}},}{\left.\frac{\partial f_{0}(T)}{\partial T}\right|_{\left(T_{0}, 0\right)} ^{f}\left(\left.T_{0} \frac{\partial f_{2}(T)}{\partial T}\right|_{\left(T_{0}, 0\right)}-2 f_{2}\left(T_{0}\right)\right)+f_{4}\left(T_{0}\right)} .
$$

Here

$$
f_{2 n}(T)=\left.\frac{1}{(2 n) !} \frac{\partial^{2 n} f\left(T, \mu_{B}\right)}{\partial\left(\mu_{B} / T\right)^{2 n}}\right|_{(T, 0)} .
$$

The coefficients $\kappa_{2}^{f}$ and $\kappa_{4}^{f}$ are calculated in the CMF model for the pressure $P$, the energy density $\varepsilon$, and the entropy density $s$ as functions of the temperature $T$. The CMF model predictions are in a reasonable agreement with recent LQCD calculations [164]. The rather low values of $\kappa_{2}^{f}$ and $\kappa_{4}^{f}$ suggest also small curvatures of the lines of constant physical observables in the temperature region studied here. Effects of the finite chemical potential are small, therefore these lines are almost horizontal in the $T-\mu_{B}$ plane. The coefficients for the entropy and energy density indicate that $\kappa_{2}^{s}<\kappa_{2}^{\varepsilon}$, meaning a decrease of the entropy density along the lines of constant energy density.

\section{V.3 The CMF model phase diagram}

Two order parameters, the chiral condensate $\sigma$ and the Polyakov loop $\Phi$, plus the interacting baryon octet within the $\mathrm{SU}(3)$-flavor $\sigma$-model permit four different phases within the CMF model. These phases are characterized as:

- A dilute gas of interacting hadrons;

- A hadronic liquid - a dense hadronic phase, the transition from the hadron gas to the hadronic liquid is the nuclear liquid-vapor phase transition. Quarks start to appear in the hadronic liquid, but they are negligible; 

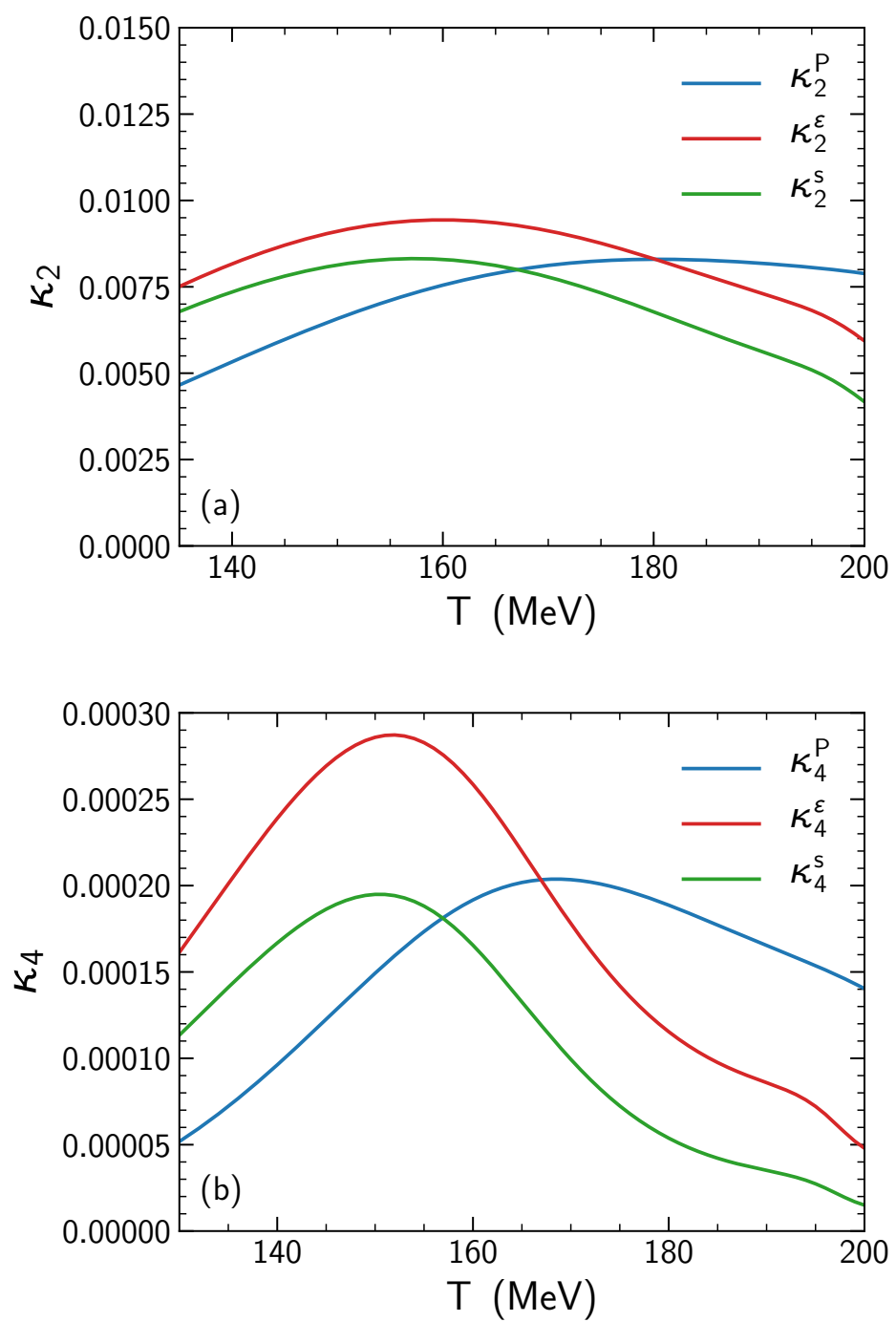

Figure V.5: Second $\kappa_{2}^{f}$ (a) and fourth $\kappa_{4}^{f}$ (b) coefficients of line of constant pressure $P$, energy density $\varepsilon$ and entropy density $s$ versus temperature $T$. Explanation and lattice data can be found at [164].

- A chirally restored phase, where the mass symmetry between the parity partners is restored. Here the quark masses are decreased, hence quarks give a sizable contribution to the thermodynamics;

- A quark matter phase, where baryonic density is carried by quark degrees of freedom. The gluon contribution is modeled by the Polyakov loop potential [181] and $\frac{1}{3} n_{q} / n_{B}=1$. 

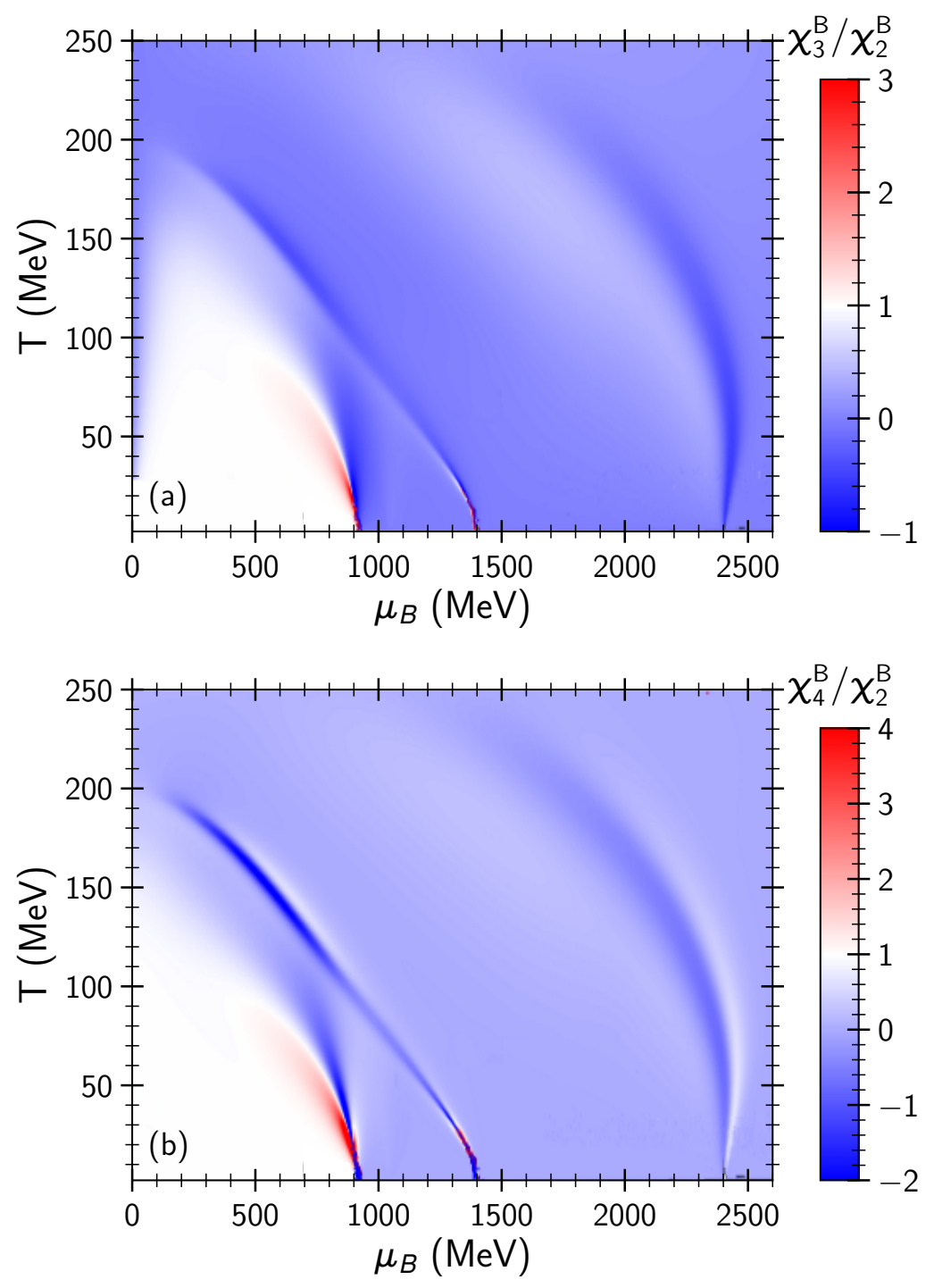

Figure V.6: Ratios of the CMF baryon number susceptibilities $\chi_{3}^{\mathrm{B}} / \chi_{2}^{\mathrm{B}}$ skewness (a) and $\chi_{4}^{\mathrm{B}} / \chi_{2}^{\mathrm{B}}$ kurtosis (b) in the baryon chemical potential - $\mu_{\mathrm{B}}$ and temperature - $T$ plane. Note the 3 distinct critical regions, with their remnants reaching from $T=0$ up to $T>200 \mathrm{MeV}$. Note the absence of calculation results for skewness at $\mu_{B} \approx 0$ and $T<100 \mathrm{MeV}$ region.

The baryon number susceptibilities $\chi_{n}^{B}$, which can be calculated using Eq. V.15, are proportional to the respective cumulants of the baryon number distribution. Higher-order baryon number susceptibilities do increase in proportion to the increasing power of the correlation length [182]. Such an increase in correlation 

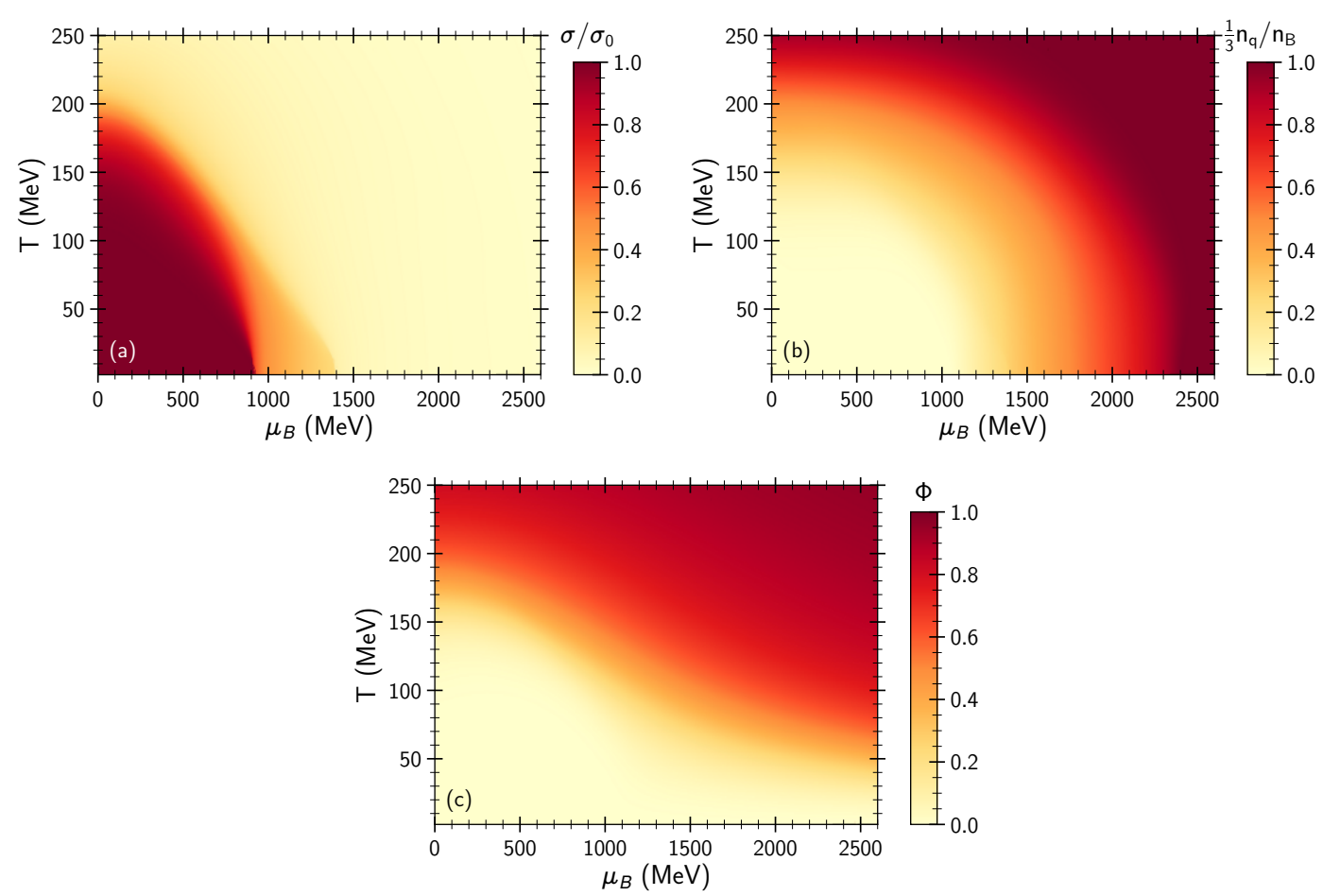

Figure V.7: The normalized non-strange chiral condensate $\sigma / \sigma_{0}$ (the sigma field) (a), the quark fraction $\frac{1}{3} n_{q} / n_{B}(\mathrm{~b})$, and the value of Polyakov loop $\Phi$ (c) of the CMF model in baryon chemical potential $\mu_{\mathrm{B}}$ and temperature $T$ plane. Note that the rather fast change of the chiral condensate appears at moderate energy densities, while deconfinement appears only at much higher energy densities/chemical potentials.

length would be reflected in large values of the 2nd and higher-order susceptibilities in the vicinity of a critical point and in the region of phase transition. Hence, these quantities are useful indicators of critical behavior in the CMF model. Deviations of $\chi_{n}^{B}$ from the corresponding baselines indicate a transformation between different phases, which is reflected usually in a non-monotonic behavior of these observables, e.g. skewness $\chi_{3}^{B} / \chi_{2}^{B}$ and kurtosis $\chi_{4}^{B} / \chi_{2}^{B}$.

The calculated skewness $\left(\chi_{3}^{B} / \chi_{2}^{B}\right)$ and kurtosis $\left(\chi_{4}^{B} / \chi_{2}^{B}\right)$ (Fig. V.6) in the CMF model exhibit non-trivial structures in the $T$ - $\mu_{B}$ phase diagram. The regions of deviations from the baseline separate regions with quantitatively different properties, which are often dubbed - "phases". Note the sharp phase boundaries indicate first order phase transitions, FOPT, these are only observable at quite moderate temperatures $T<50 \mathrm{MeV}$. The hadron phase located at both low 
temperatures $T$ and baryon chemical potential $\mu_{B}$ represents a dilute gas of interacting hadrons. There, the fluctuation measures $\chi_{2}^{B} / \chi_{1}^{B}, \chi_{3}^{B} / \chi_{2}^{B}, \chi_{4}^{B} / \chi_{2}^{B}$ are quite close to unity, consistent with the Skellam distribution baseline. The system exhibits a FOPT to a dense hadronic liquid phase with a rising chemical potential $\mu_{B} \approx 1 \mathrm{GeV}$. Here, the fluctuations are reduced due to the repulsive interactions. Quarks start to appear in moderation. The liquid phase exhibits an additional FOPT at $\mu_{B} \approx 1.5 \mathrm{GeV}$, and second order transition at $\mu_{B} \approx 2.4 \mathrm{GeV}$, which show up in the structure of the baryon number susceptibilities at these high $\mu_{B}$. The transition at $\mu_{B} \approx 1.5 \mathrm{GeV}$ is due to the restoration of chiral symmetry. The transition at $\mu_{B} \approx 2.4 \mathrm{GeV}$ is due to the quark matter phase where the baryonic density is mainly contributed by quarks. The non-monotonic behavior of the fluctuation measures $\chi_{3}^{B} / \chi_{2}^{B}$ and $\chi_{4}^{B} / \chi_{2}^{B}$ reflect the transitions. In contrast to the liquid-vapor transition, those two transitions do not change the Skellam baseline. Hence, the fluctuation measures are rather small $\lesssim 1$ before and after the "transition".

The chiral critical point of the CMF model is located at a rather low temperature $T_{C P}^{c h} \approx 17 \mathrm{MeV}$. This value is close to the critical temperature of the nuclear liquid-gas transition in the same model, the critical $\mu_{B}^{C P}$ is remarkably different, though: the appearance of the parity partners controls the dynamics of the chiral fields: as the parity partners - in the CMF model - obey the same repulsive interaction strength as the nucleons, the critical point appears at that low temperature. This phenomenon has been observed in various mean field models before.

The different phases shown in Fig. V.7 in the $T-\mu_{B}$ plane are related to the chiral field $\sigma$ and the quark fraction. The chiral field is close to its vacuum value, $\sigma=\sigma_{0}$, at the hadron gas region, here the quark fraction is close to zero, as expected. Both observables deviate from their vacuum values at higher densities and temperatures only.

The chiral field drops off more slowly at $\mu_{B}=0$ than seen in lattice QCD calculations, where the chiral field rapidly drops around $T=155 \mathrm{MeV}$. The reason for this discrepancy is due to the fact that in the present CMF model the thermodynamics at these temperatures are strongly influenced by the many hadronic states which are not coupled to the chiral fields here. Baryon resonances like the $\Delta$ or mesons like $\pi$ are significantly abundant at these temperatures and thus may significantly affect the values of chiral field. The coupling of the chiral 


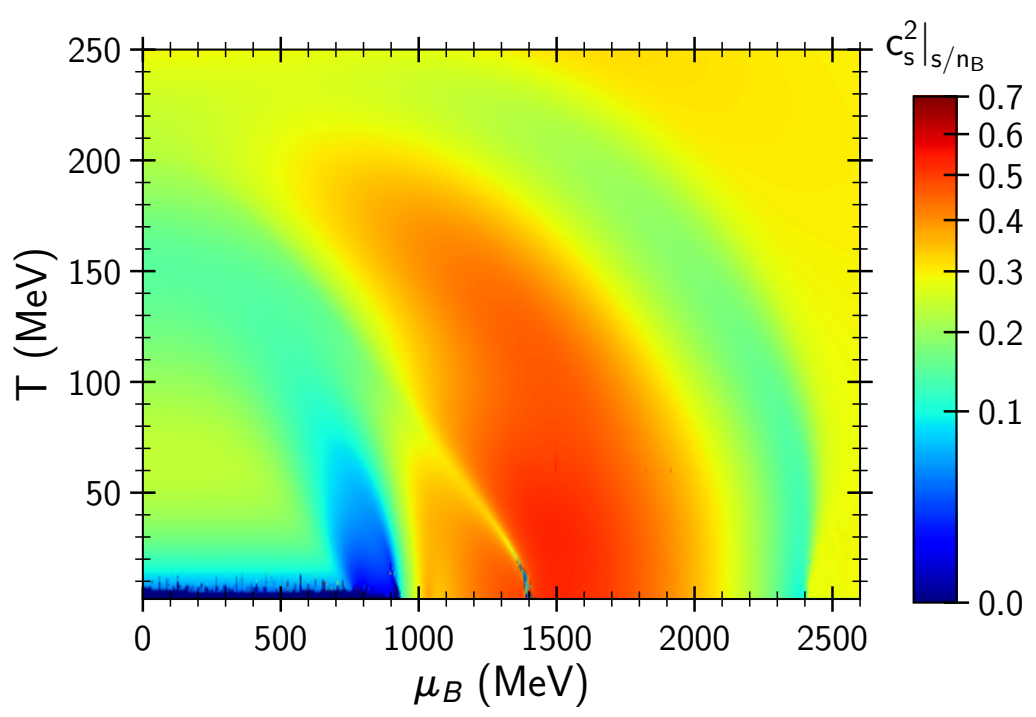

Figure V.8: The CMF model results for the square of the isentropic speed of sound $c_{s}^{2}=\left(\frac{\partial p}{\partial \epsilon}\right)_{s / n_{B}}$, calculated along lines of constant total entropy density per net baryon density $s / n_{B}=$ const, as from Eq. V.20.

field to the hadronic states beyond the baryon octet was studied in [183], it was found that this scenario brings the chiral transition to lower temperatures.

The speed of sound is another important signature for the simulations of the dynamics of heavy ion collisions and neutron star mergers. $c_{s / n_{B}}^{2}$ presents a derivative $c_{s / n_{B}}^{2}=\frac{\partial P}{\partial \varepsilon}$ at constant $S / A=s / n_{B}$ entropy per baryon, that allows to estimate the speed of propagation of sound-like excitations in non-dissipative hydrodynamic evolution. The isentropic speed of sound can be calculated as [184]:

$$
c_{s / n_{B}}^{2}=\frac{n_{B}^{2} \partial_{T} s-2 n_{B} s \partial_{\mu_{B}} s+s^{2} \partial_{\mu_{B}} n_{B}}{(\epsilon+P)\left(\partial_{\mu_{B}} n_{B} \partial_{T} s-\left(\partial_{T} n_{B}\right)^{2}\right)}
$$

The partial derivatives with respect to the chemical potential and to the temperature are performed at constant temperature and at constant chemical potential, respectively. The calculated speed of sound shows three local minima which correspond to the three locally softest points of the EoS. These three minima correspond to phase boundaries, where the baryon number susceptibilities present a non-monotonic behavior. Note, that the speed of sound reaches quite large values, $c_{s}^{2} \approx 0.7$, in the higher density region of nuclear matter. This high speed of sound results due to the strong repulsion between the baryons, before the onset of deconfinement. Thereafter, the vector repulsion and baryon excluded volume 
cease, as such terms have not been predicted for the quarks [185, 186].

\section{V.4 Taylor expansion at large densities and the role of HRG particle list}

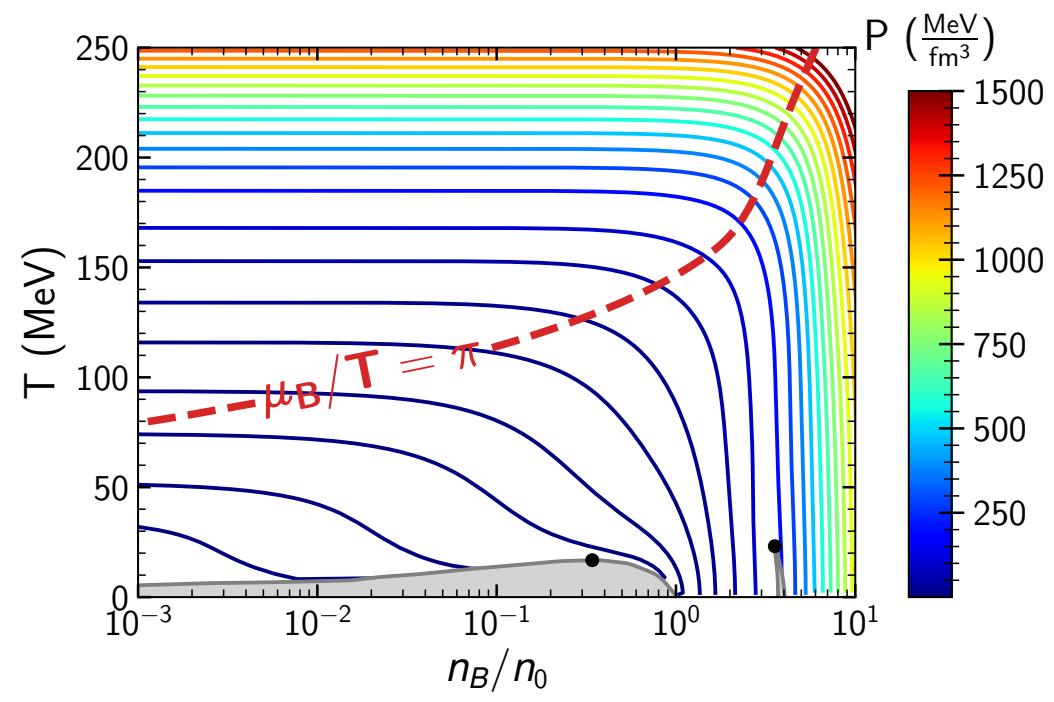

Figure V.9: Lines of constant pressure over $n_{B}-T$ plane, color indicates the value of pressure $P$ at the contour, baryon density $n_{B}$ is normalized to the nuclear saturation density $n_{0}$. The red dashed line indicates the line of $\mu_{B} / T=\pi$, this line lies in a proximity of abrupt tilt of constant pressure lines. The grey shaded regions illustrate mixed regions produced by nuclear liquid-vapor and chiral phase transitions. Black dots indicate critical endpoints.

The LQCD studies of the QCD phase diagram are mainly carried out via the Taylor expansion in series of $\mu_{B} / T$ [187]. The current estimates of a radius of convergence of the expansion suggest $R_{\mu_{B} / T} \sim 3$ at $T \gtrsim 135 \mathrm{MeV}[164,188]$, which limits the applicability of the lattice studies of matter at large baryon densities. The CMF model allows studying physics also at much higher $\mu_{B}$. On Fig. V.9 (left) the lines of constant pressure - the isobars - are presented across the $n_{B}-T$ plane. Additionally, the $\mu_{B} / T=\pi$ line is plotted, which is a convergence radius limitation that may arise due to the Roberge-Weiss [189] singularity at imaginary values of chemical potential, $\mu_{B} / T=i \pi . R_{\mu_{B} / T} \approx \pi$ values have been suggested by means of a cluster expansion model (CEM) analysis of LQCD data [188]. 
Fig. V.9 (left) illustrates that up to $\mu_{B} / T \approx \pi$ the pressure changes insignificantly with isobars being virtually horizontal. In this region, the pressure is dominated by particles and antiparticles almost on the same level and for $T \gtrsim 100 \mathrm{MeV}$ is dominated by mesons that do not carry baryon charge. For temperatures $T>100 \mathrm{MeV}$ the convergence region significantly widens due to the increasing dominance of mesonic degrees of freedom which smears out the effects of finite $\mu_{B}$. At sufficiently large values of $T$ the matter is mostly composed by quarks and gluons with the latter not carrying the baryon charge. For quark-gluon matter, the radius of convergence is expected to be limited by the Roberge-Weiss transition although the answer may depend on the exact nature of this transition [190]. The presented behavior suggests that the Taylor expansion in $\mu_{B} / T$ in the region $\mu_{B} \approx 0$ only allows to explore the matter where effects of finite baryon densities are small and the pressure is dominated by mesons and gluons, the pressure of particles that carry the baryon charge is on the same level as of antiparticles with a negative baryon charge. At temperature $T \approx 150 \mathrm{MeV}$ the expansion is reliable up to $n_{B} \lesssim 2 n_{0}$ and decreases for smaller temperatures. This finite region of baryon density limits usage of the LQCD results and makes it inapplicable to low energy heavy ion collisions and neutron star mergers. Therefore, hydrodynamical simulations of such systems require the EoS produced by different means.

We also examine the role of mesonic and resonance degrees of freedom in the hydrodynamic evolution of a hot, strongly interacting fireball. The relevance of mesons and hadronic resonances in the expansion of the fireball created in heavy ion collisions was pointed out a long time ago [191]. However, the role of these thermally excited states in hot hadronic matter created in neutron star mergers is still under investigation $[4,6]$. Figure V.9 presents isentropes - the lines of constant entropy per baryon $S / A=$ const - which represent trajectories in the $n_{B}-T$ plane of an ideal hydrodynamic expansion. The trajectories show that an omission of mesons and baryonic resonances leads to an artificial increase of temperature in the hydrodynamic evolution.

\section{V.5 Application to heavy-ion collisions}

The presented EoS is used as input for hydrodynamical simulations of both heavy-ion collisions and neutron star mergers. To illustrate which regions of the phase diagram can be reached in collisions at low and moderate collision 


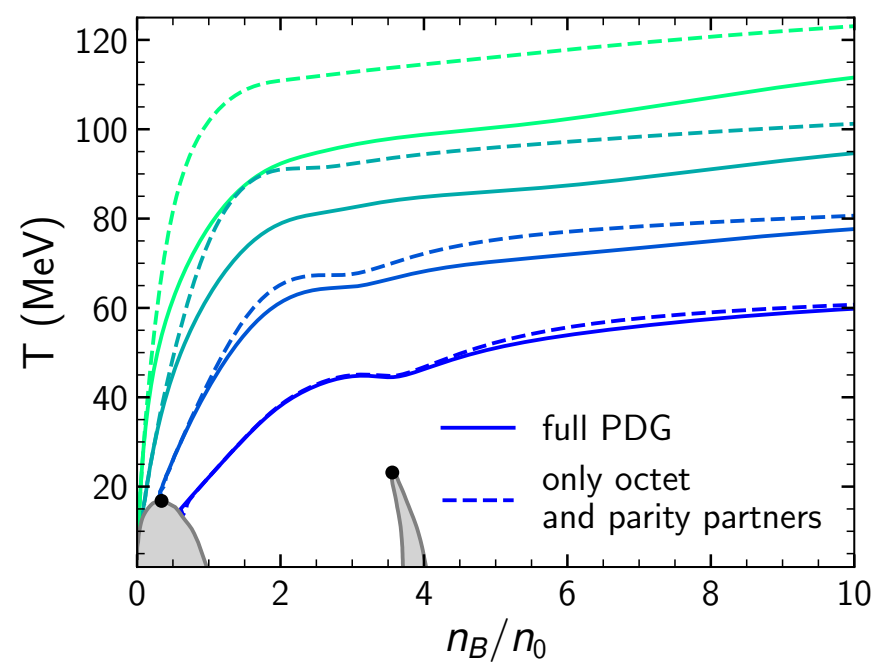

Figure V.10: Isentropic trajectories, lines of constant entropy per baryon $S / A$, calculated within the CMF model with the full particle list (solid) and only with the stable baryons+quarks (dashed) where mesons and resonances are neglected. Note the increase of temperature for the isentropes where mesons and resonances are neglected.

energies, the stationary 1-dimensional Taub adiabat model is used [192, 191, 193]. The expansion is described at lines of constant entropy per baryon $S / A=$ const (isentropes). These lines depict the isentropic matter evolution of ideal fluid dynamics at different collision energies.

The entropy is produced in the earliest stage of a heavy ion collision by the violent shock compression [194]. During the system's expansion, there is only a moderate increase of entropy due to the rather small viscosity [195, 196], hence, an isentropic expansion scenario is a reasonable approximation [197].

The expansion of the equilibrated matter then continues until the system becomes so dilute that the chemical, as well as the kinetic freeze-out, occur and the chemical composition is fixed.

The entropy per baryon $(S / A)$ is calculated in the 1-dimensional stationary scenario of central heavy ion collisions - the two colliding slabs of cold nuclear matter [198, 191, 199, 200, 201, 202, 194] conserve the baryon number, energy and momentum across the shock front in accord with the relativistic Rankine-Hugoniot equation (Taub adiabat), RRHT, [192, 193]. Thus, the produced entropy is directly 


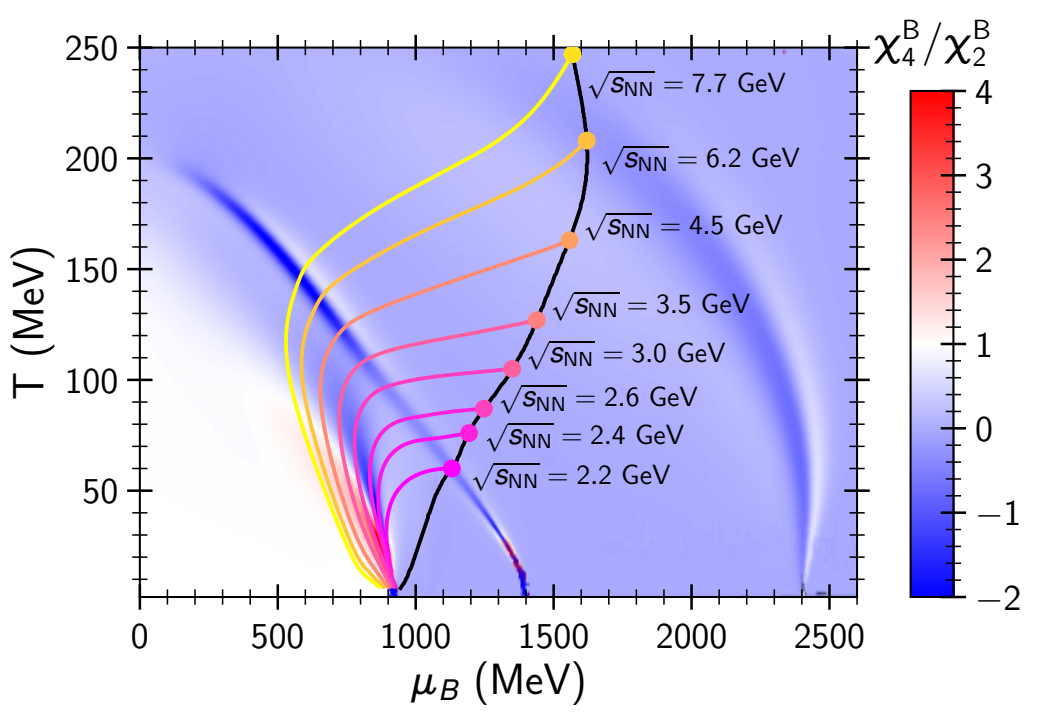

Figure V.11: Evolution of heavy-ion collisions in the high baryon density region of the $T-\mu_{B}$ phase diagram for different collision energies. Black line - Taub adiabat which describes the initial state of heavy ion collisions as an implicit function of $\sqrt{s_{\mathrm{NN}}}$. Colored lines - isentropic lines of constant entropy per baryon $S / A$ at different bombarding energies $\sqrt{s_{N N}}$ respectively. See Table V.3 for details.

\begin{tabular}{cccccc}
$\sqrt{s_{N N}}(\mathrm{GeV})$ & $E_{\mathrm{lab}}(\mathrm{GeV})$ & $S / A$ & $T(\mathrm{MeV})$ & $n_{B} / n_{0}$ & $\frac{1}{3} n_{q} / n_{B}$ \\
\hline \hline 2.2 & 1.6 & 2.8 & 60.0 & 2.8 & 0.11 \\
2.4 & 2.1 & 3.5 & 76.0 & 3.4 & 0.20 \\
2.6 & 2.7 & 4.1 & 87.0 & 3.9 & 0.26 \\
3.0 & 3.9 & 5.3 & 105.0 & 4.9 & 0.35 \\
3.5 & 5.6 & 6.6 & 127.0 & 6.0 & 0.46 \\
4.5 & 9.9 & 8.4 & 163.0 & 8.6 & 0.70 \\
6.2 & 19.6 & 10.7 & 208.0 & 14.8 & 0.96 \\
7.7 & 30.7 & 13.1 & 247.0 & 18.7 & 1.00
\end{tabular}

Table V.3: Initial state properties obtained from the one-dimensional stationary case of the central heavy ion collision - Taub adiabat $[192,193]$. The entropy per baryon $S / A$, temperature $T$, the initial baryon density $n_{B} / n_{0}$ and the quark fraction $\frac{1}{3} n_{q} / n_{B}$ are presented for various colliding energies.

associated with the collision energy. The thermodynamic properties across the 

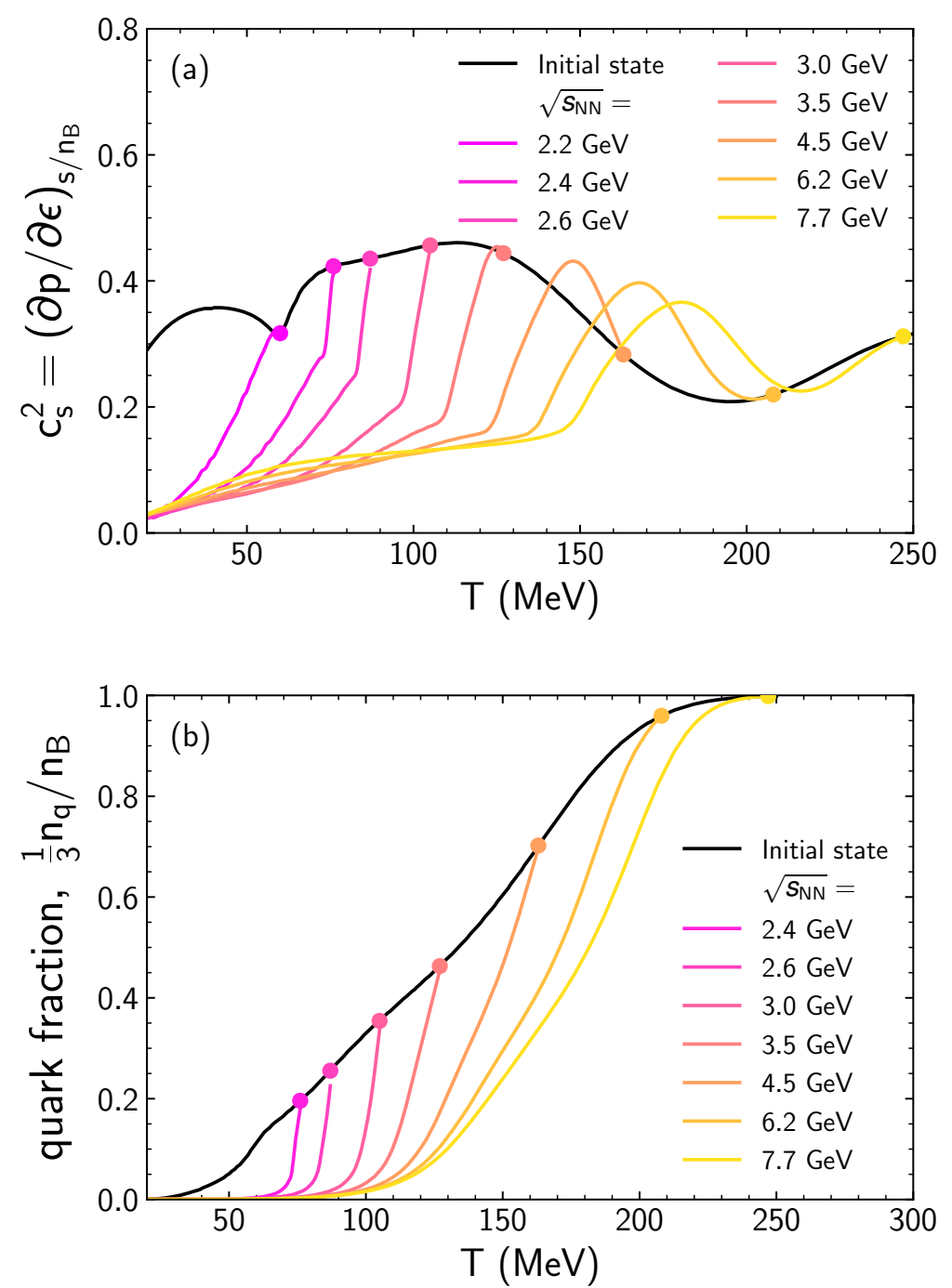

Figure V.12: Speed of sound at constant entropy per baryon $c_{s}^{2}$ (a) and quark fraction (b) along the isentropes as functions of temperature $T$. Colored lines correspond to different collision energies (initial entropy per baryon $S / A$ ), black solid line correspond to the initial speed of sound and the quark fraction respectively. Isentropes are the same as in Fig. V.11. See Table V.3 for details.

shock front are described by the RRHT-equation

$$
\left(P_{0}+\varepsilon_{0}\right)\left(P+\varepsilon_{0}\right) n^{2}=\left(P_{0}+\varepsilon\right)(P+\varepsilon) n_{0}^{2},
$$

where $P_{0}, \varepsilon_{0}$ and $n_{0}$ correspond to the initial pressure, energy density, and baryon density in the local rest frame of each of the two slabs. The two symmetric slabs 
consist of the nuclear matter in the ground state, $P_{0}=0, \varepsilon_{0} / n_{0}-m_{N}=-16$ $\mathrm{MeV}$ and $n_{0}=0.16 \mathrm{fm}^{-3}$. With any known relation $P=P(\varepsilon, n)$, Eq. V.21 can be solved. Furthermore, the collision energy is related to the created density as follows:

$$
\gamma^{\mathrm{CM}}=\frac{\varepsilon n_{0}}{\varepsilon_{0} n}, \gamma^{\mathrm{CM}}=\sqrt{\frac{1}{2}\left(1+\frac{E_{\mathrm{lab}}}{m_{N}}\right)} .
$$

Here, $\gamma^{\mathrm{CM}}$ is the Lorentz gamma factor in the center of mass frame of the heavy ion collisions and $E_{\text {lab }}$ is the beam energy per nucleon in the laboratory frame of a fixed target collision. This relation can be obtained from the full stopping condition [191, 199, 200, 201, 202, 194, 203]. The initial state thermodynamics (density, temperature and entropy) of the hot, dense participant matter is obtained from Eqs. (V.21) and (V.22) as a function of the collision energy. The known initial entropy yields the lines of constant entropy which give the trajectories of the heavy ion collisions in the phase diagram.

The predicted isentropic expansion trajectories are shown in the $T-\mu_{B}$ plane phase diagram in Fig. V.11.

Note that 1-dimensional stationary RRHT-adiabat scenario predicts a very strong compression and heating already at intermediate bombarding energies. The heavy ion participant system crosses the weak chiral transition predicted by the present $\mathrm{CMF}$ model already at $E_{\text {lab }} \approx 2 \mathrm{~A} \mathrm{GeV}$, i.e. at the GSI's SIS18 accelerator facility. Here, the specific total entropy is predicted to reach $S / A \approx 3$, in accord with previous RMF-calculations [202] which also used the 1-D RRHTscenario. The $T-\mu_{B}$ values, $T \approx 70 \mathrm{MeV}, \mu_{B} \approx 1.2 \mathrm{GeV}$, with net baryon densities $n_{B} / n_{0} \approx 3$, reached here in heavy ion collisions, coincide with the $T-\mu_{B}$ values reached in binary neutron star collisions, as recent general relativistic fully 3+1-dimensional hydrodynamical calculations have confirmed [204, 205] for the gravitational wave event GW170817. At these temperatures and densities, $T \approx 70$ $\mathrm{MeV}$ and $n_{B} / n_{0} \approx 3$, the RRHT model predicts that about $20 \%$ of the dense matter is already transformed to deconfined quarks.

At $E_{\text {lab }}=5.6 \mathrm{~A} \mathrm{GeV}, \sqrt{s_{\mathrm{NN}}}=3.5 \mathrm{~A} \mathrm{GeV}$ roughly $40 \%$ of the CMF-matter is in the quark state in the RRHT model - a prerequisite for hot quarkyonic matter. Hence, this energy is of great interest: here the matter starts to be dominated by quarks, rather than by in-medium baryons, at $T>100 \mathrm{MeV}$ and $\mu_{B} \gtrsim 1.5 \mathrm{GeV}$. This is predicted by the present CMF model when using the 1-D RRHT ideal hydrodynamics. This model predicts that the quarkyonic transition is crossed 
also at higher energies, using the isentropic expansion of the matter at specific total entropy $S / A>6$. Non-equilibrium viscous effects may increase the specific entropy of the system. However, pre-freezeout radiation, e.g. of Kaons and other hadrons with small scattering cross-sections which can escape early from the semi-equilibrated baryon-rich, dense system can considerably lower the specific entropy during the expansion. So an answer to the question of whether the local entropy per baryon increases or decreases during the time evolution awaits more detailed microscopic/macroscopic modeling.

Hence, heavy ion fixed target experiments of SIS at FAiR and SPS at CERN as well as STAR BES program at RHIC probe temperatures from $50<T<280$ $\mathrm{MeV}$ and chemical potentials from $500<\mu_{B}<1700 \mathrm{MeV}$ for the collision energy range $\sqrt{s_{\mathrm{NN}}}<10 \mathrm{GeV}$ considered here. In this region, the CMF model does not show an additional phase transition, but the remnants of the nuclear liquid-vapor transition at $T \approx 20 \mathrm{MeV}$. The chiral transition at larger chemical potentials can influence the dynamical evolution, too. The present results suggest that heavy-ion collisions mostly probe regions where the nuclear matter liquid-vapor critical point dominates - hence, the observed baryon fluctuations are largely due to remnants of the nuclear liquid-vapor phase transition. This had been suggested also in previous works $[206,180,155,207]$. The $\mathrm{CP}$ associated with the chiral symmetry restoration in the $\mathrm{CMF}$ model lies at $\mu_{B} \approx 1.5 \mathrm{GeV}$ and $T \approx 17 \mathrm{MeV}$. This high density region is, to the best of our knowledge, reachable only in the interiors of neutron stars, NS, and in binary general relativistic NS mergers [208, 209, 205, $210,4]$.

Fig. V.12 presents the square of the isentropic speed of sound, at fixed specific entropy, $c_{s}^{2}$ and the quark fraction as functions of the temperature for the studied collision energies, i.e. it shows how respective observable quantities evolve during the cooling of the system while it expands. Those isentropic lines which belong to $\sqrt{s_{N N}}>4.5 \mathrm{GeV}$ probe the softest point of the EoS, which is attributed to the chiral symmetry restoration. At this energy region, there are strong local maxima and minima of the speed of sound squared after which $c_{s}^{2}$ rapidly increases during the expansion due to the decrease of the quark fraction, as a result of the rapid appearance of baryons, the EoS stiffens quickly due to the hard-core baryon-baryon repulsion. For collision energies $\sqrt{s_{N N}}<4.5 \mathrm{GeV}$ the initial state is not dominated by quarks, hence, the system starts to expand at rather high values of $c_{s}^{2}$, which then monotonously decrease during the expansion, as a result 
of the diminishing repulsion between the baryons.

\section{V.6 Application to neutron stars}

The densities in a neutron star interior can exceed several times the nuclear matter saturation density. At these high densities, the lack of detailed knowledge of the equation of state and the appropriate microscopic degrees of freedom is similar as in relativistic heavy ion collisions discussed above. The discussion of the role of hyperonic, quarkyonic, and strange quark degrees of freedom at these NS densities is ongoing.

The CMF model can be employed directly to describe neutron star matter. Here we work without any changes to the coupling constants and parameters used to describe the $\mu_{B}=0$ LQCD results. The temperatures isolated in neutron star interiors are negligibly small, in comparison to what we observe in heavy ion collisions and hot QCD scales. The calculations here are done in the limit $T=0$. In contrast to ordinary isospin symmetric nuclear matter, neutron star matter is in $\beta$-equilibrium which preserves the total electric charge neutrality of NS matter and locally ensures stability with respect to $\beta$-decay. As a consequence, strangeness and hypercharges assume finite values. These constraints require the presence of leptons.

In the case when two conserved charges are present, namely, electric and baryonic, a phase transition is non-congruent $[211,212,213]$ as a result of global charge conservation. The CMF-model predicts only one, chiral phase transition for matter in $\beta$-equilibrium. This transition is non-congruent and results only in a moderate increase of baryon density between the two phases.

Figure V.13 depicts the CMF model predictions of the relative particle densities of all different particle species present inside a CMF neutron star, at $T=0$, as a function of baryon chemical potential. One feature of the present CMF model is the absence of baryon resonances (Deltas etc. and of hyperons) even though they are included in the CMF model. Their total absence in the present CMF model calculation at $T=0$ is due to the very strong hard-core repulsion by the excluded volume corrections.

The calculated EoS at $T=0$ can be used as the input for the TolmanOppenheimer-Volkoff (TOV) equation, which allows the relation between the mass with the radius of any static, spherical, gravitationally bound object [214, 215], 

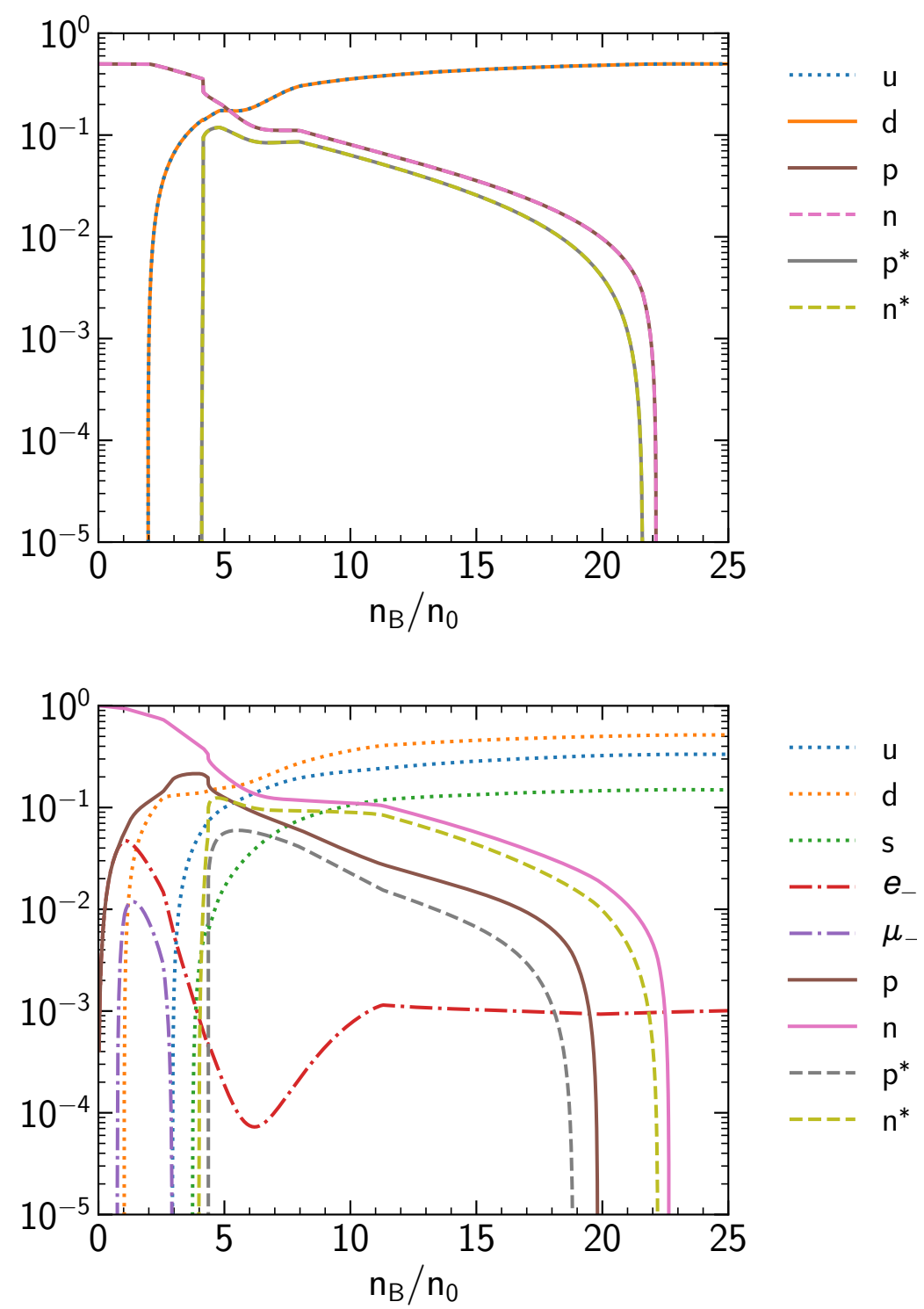

Figure V.13: Particle density ratios to the density of baryons $n_{i} / n_{B}$ at $T=0$, for quarks a factor of $1 / 3$ is used, presented as functions of baryon density $n_{B}$. The CMF-results are obtained for isospin symmetric matter (top) and in $\beta$-equilibrium (bottom).

i.e. here a static neutron star, NS. The outer layers of a neutron star presumably consist of mostly neutron rich nuclei and clusters in chemical and $\beta$ - equilibrium. Those nuclei are not yet included in the CMF model. Hence, another input for the EoS of the NS crust is needed. Here, we use the classical crust-EoS [176] matched 
to the CMF-EoS at $n_{B} \approx 0.05 \mathrm{fm}^{-3}$.

Figure V.14 presents the results on the NS mass-radius relations obtained by solving the TOV equation with the present CMF EoS, matched just to that crust-EoS. The total fraction of the star's mass which consists of light and strange quarks is presented in color-code. The most massive stable solution of the TOV equation contains only $<30 \%$ deconfined quarks, i.e. for lighter NS only a small fraction of the star's mass originates from deconfined quark matter. If the quark fraction is increased above $30 \%$ the stars become unstable. The central density of the stable stars can never exceed $n_{B}=6 n_{0}$, as shown in the lower part of Fig. V.14. Here again, the maximum mass indicates the "last stable star". The continuous slow transition from NS matter to a sizable deconfined quark phase implies a smooth appearance of quarks in the star structure. This does prohibit a strict separation between a quark core and the hadronic interior of the star. This is a CMF result due to the Polyakov loop implementation of the deconfinement mechanism and no vector repulsion among quarks. The absence of quark repulsion along with the smooth appearance of free quarks disfavors the "second family" of neutron stars since in the quark phase EoS is soft and cannot support a strong gravitational compression. Although LQCD data disfavors repulsive forces for quarks, there is active discussion in the astrophysical community on the role of the vector repulsion in the physics of neutron stars [216, 217, 218]. An approach with density dependent quark vector coupling is developed in [219] where it is argued that the repulsion arises from non-perturbative gluon exchange.

The Quarkyonic Matter-model yields similar to our results [220]. There the deconfinement is realized by the appearance of the quarks from inside of the Fermi sea while the hadrons reside exclusively on the surface shell in momentum space. A similar approach to deconfinement was suggested in [221, 222], however, there the produced mass-radius diagram differs from the CMF-model [2] due to the different realization of the chiral interactions.

The response of a neutron star to non-spherical gravitational fields is reflected in the tidal deformability coefficient $\lambda$ [223], which depends strongly on the EoS. During the inspiral phase of the binary neutron star merger, both neutron stars experience tidal deformations induced by the other respective accompanying neutron star partner. The tidal deformability $\lambda$ is a measure of the induced quadruple moment $Q_{i j}$ in a response to the external tidal field $\mathcal{E}_{i j}$ :

$$
Q_{i j}=-\lambda \mathcal{E}_{i j} .
$$


$\lambda$ is directly proportional to the second Love number $k_{2}$ :

$$
\lambda=\frac{2}{3} k_{2} R^{5} .
$$

For convenience, usually the dimensionless tidal deformability $\Lambda$ is presented as:

$$
\Lambda=\frac{\lambda}{M^{5}}=\frac{2}{3} k_{2}\left(\frac{R}{M}\right)^{5}
$$

Here, $M$ and $R$ are the mass and radius of the neutron star. A proper value of $\Lambda$ is important for the description of the inspiral stage during the merger of two neutron stars.

Various estimates of $\Lambda$ emerged after the detection of GW170817 by the LIGO and the Virgo collaborations [224]. Ref. [225] argued that for a $1.4 M_{\odot}$ neutron star the tidal deformability and star radius are constrained to $\Lambda_{1.4 M_{\odot}}>120$ and $R_{1.4 M_{\odot}}<13.6 \mathrm{~km}$. It was concluded by means of a Bayesian analysis that for a $1.4 M_{\odot}$ star the deformability should be $375.5<\Lambda_{1.4 M_{\odot}}<800$ and the radius is at $12.00<R_{1.4 M_{\odot}}<13.45 \mathrm{~km}$, with respective $2 \sigma$ confidence levels, see Ref. [226]. A recent analysis by the LIGO and Virgo collaborations [227, 228] provides detailed constraints, by using a Bayesian analysis based on the reproducing of the details of the gravitational wave signal. 

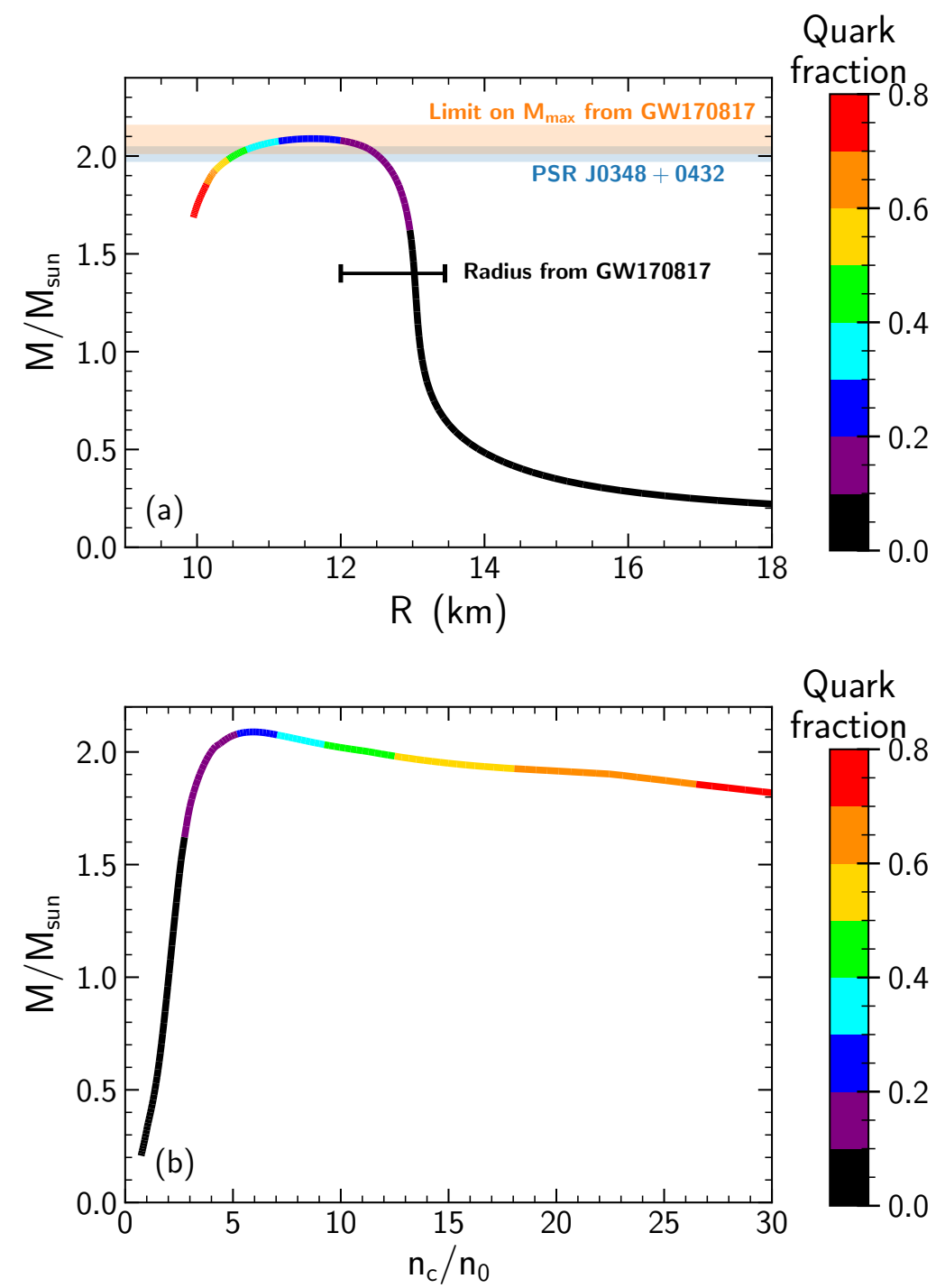

Figure V.14: The CMF mass-radius diagram (a) for neutron stars is shown as calculated within the CMF model. The CMF neutron star mass is shown as a function of central density $n_{c}$ normalized to saturation density $n_{0}$ (b). Color indicate the fraction of the star coming from the quarks. 
CHAPTER V. A UNIFIED APPROACH FOR QCD MATTER: CHIRAL
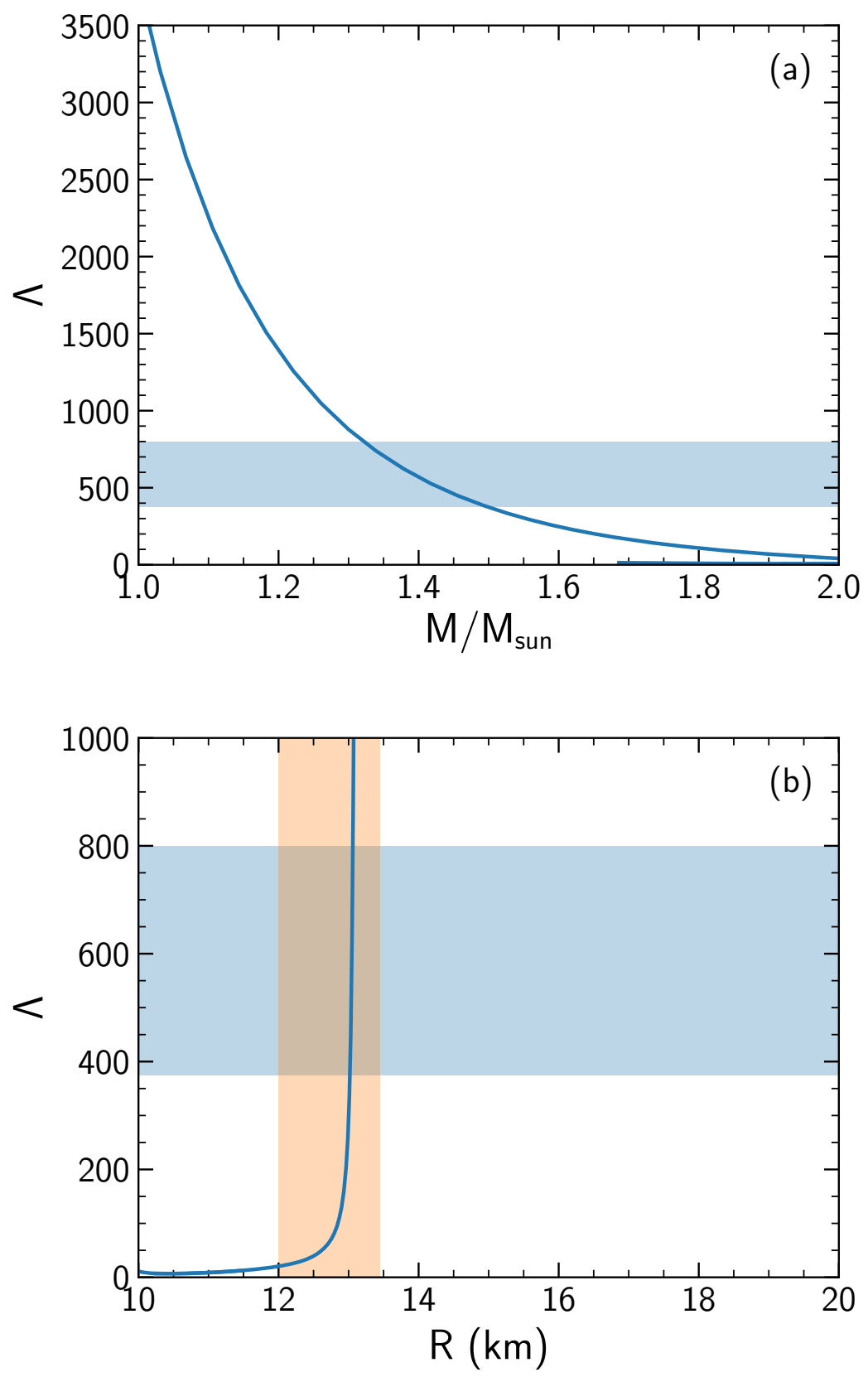

Figure V.15: Tidal deformability $\Lambda$ as function of NS mass (a) and radii (b). Blue bands correspond to $\Lambda$ constraints of NS with $M=1.4 M_{\text {sun }}$, and yellow bands constraints on the radius of NS with $M=1.4 M_{\text {sun }}[226]$. 


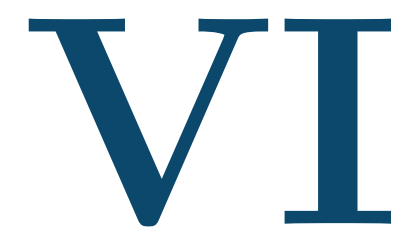

\section{Repulsive properties of hadrons in lattice QCD data and neutron stars}

In this chapter different scenarios of hadron hard-core interactions in the CMF model are studied. Here the CMF model presented before is used with varied excluded volume parameters of non-strange baryons, strange baryons, non-strange mesons, and strange baryons. The values of the hadron excluded volume parameters are constrained by the available lattice QCD data for the second order susceptibilities $\chi_{i j}^{11}$ of baryon, electric, and strangeness charges, $B, Q$, and $S$. It is found that $\chi_{i j}^{11}$ are especially sensitive to the short-range repulsive interactions of hyperons. Decreasing the hyperons size, as compared to the size of the non-strange baryons, does improve significantly the agreement of the CMF model results with the Lattice QCD data. The comparison with lattice QCD data suggests that strange baryons, non-strange mesons, and strange mesons have significantly smaller excluded volumes than non-strange baryons. The CMF model with these modified hadron volumes allows for a mainly hadronic description of the QCD susceptibilities significantly above the chiral pseudocritical temperature. This improved CMF model which is based on the lattice QCD data has been used to study the properties of both cold QCD matter and neutron star matter. The phase structure in both cases is essentially unchanged. The hyperons survive deconfinement to higher densities than non-strange hadrons. The neutron star maximal mass remains close to $2.1 M_{\odot}$ and the mass-radius diagram is only 
modified slightly due to the appearance of hyperons and is in agreement with astrophysical observations.

This chapter is based on the research performed by the author of this thesis and published in Ref.[9].

\section{VI.1 Lattice data comparison}

Important information about the phase structure at vanishing and finite baryon densities can be extracted from the fluctuations and correlations of conserved charges which are characterized by the susceptibilities [229], these quantities are sensitive to the effective degrees of freedom and their interactions. The critical regions of the QCD phase diagram are characterized by a non-monotonic behavior of these susceptibilities [182]. However, the lattice results for vanishing chemical potentials show a smooth transition between two baselines, a non-interacting ideal Hadron Resonance Gas (HRG) and weakly interacting quark-gluon matter in the region of temperatures between $100-250 \mathrm{MeV}[177,24]$. From duality arguments it should be possible to describe this transition, up to a certain point, in terms of a strongly interacting gas of hadronic degrees of freedom. Such a study is usually carried out by phenomenological models where the effective degrees of freedom and their interactions are given as input.

Here we employ an effective hadron-quark model, the CMF model, which already incorporates a smooth transition between hadrons and quarks. First, different second order susceptibilities of conserved charges are calculated within the CMF model and a comparison with available lattice data is presented. This is done to highlight the importance of different repulsive interactions for nonstrange and strange baryons and mesons for the extracted susceptibilities. A thermal model analysis of experimental hadron yields already provides indications that flavor dependent interactions in the EV-HRG are important to describe the transition region properly [117]. This idea was then further extended by a brief analysis of LQCD on susceptibilities in Ref. [231]. The results indicated that susceptibilities which involve the baryon $B$ and strange $S$ charges are sensitive to the repulsive interactions amongst strange hadrons. Using the CMF model, we can study how the LQCD data can be described by a proper modelling of the repulsive short range interactions represented by the effective excluded volume sizes of the hadrons. 


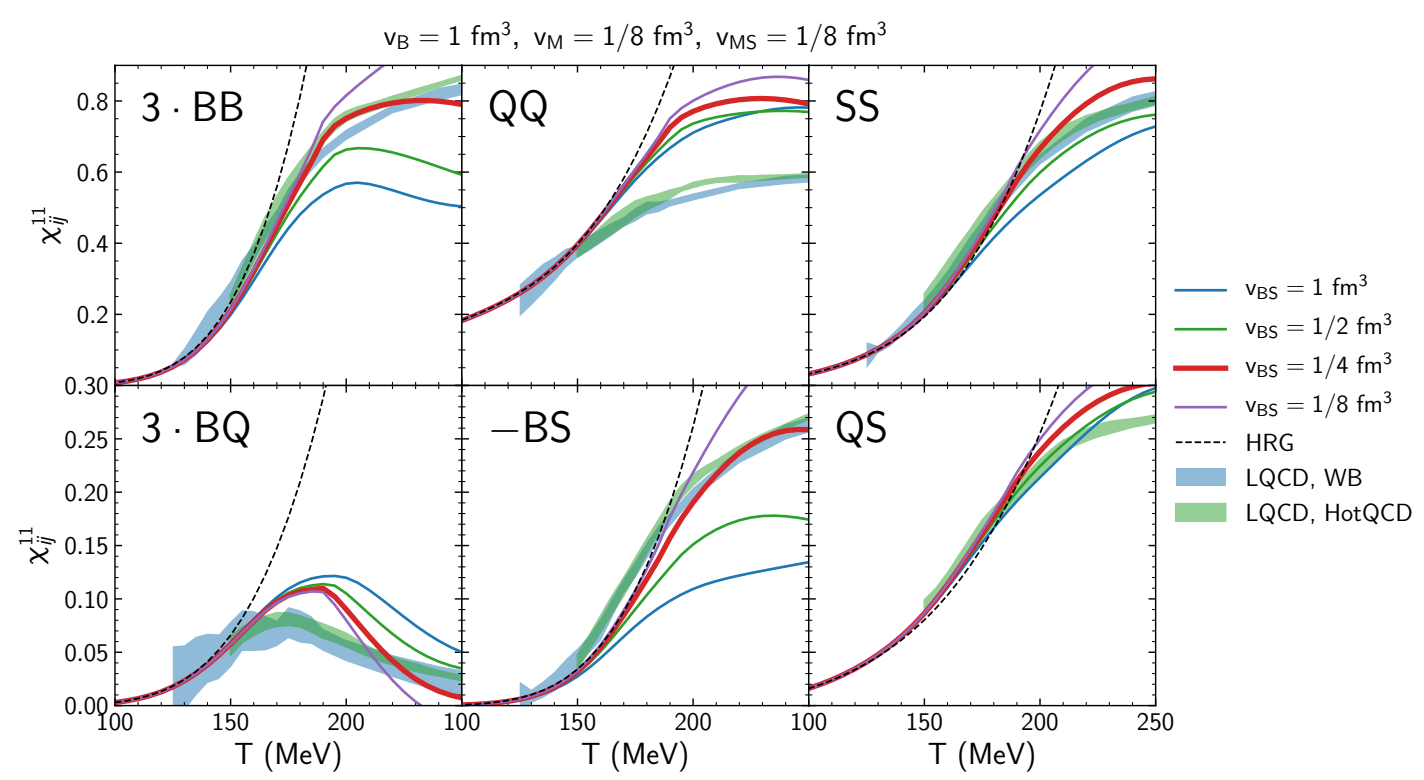

Figure VI.1: Second order susceptibilities $\chi_{i j}^{11}$ as functions of temperature $T$ for various excluded volume parameters of strange baryons $v_{\mathrm{BS}}$. The predictions of the CMF model, solid lines, are compared with available results of lattice QCD calculations by the Wuppertal-Budapest collaboration [162] and HotQCD collaborations [230], blue and green colorbands, respectively. The HRG results are also presented by the black dashed lines. All susceptibilities related to the baryon number and strangeness show a strong sensitivity to the hyperon EV. The line which best fits to the lattice data is presented in bold for $v_{\mathrm{BS}}=1 / 4 \mathrm{fm}^{3}$.

The conserved charge susceptibilities are related to the Taylor series expansion in powers of baryon, electric, and strange chemical potentials, $\mu_{B}, \mu_{Q}$, and $\mu_{S}$, of the thermodynamic pressure of matter at vanishing chemical potentials [187]. The pressure expansion to finite chemical potentials takes the form:

$$
P=P_{0}+T^{4} \sum_{i, j, k} \frac{1}{i ! j ! k !} \chi_{B, Q, S}^{i, j, k}\left(\frac{\mu_{B}}{T}\right)^{i}\left(\frac{\mu_{Q}}{T}\right)^{j}\left(\frac{\mu_{S}}{T}\right)^{k}
$$

where $P_{0}$ is the pressure at vanishing chemical potentials, and $\chi_{B, Q, S}^{i, j, k}$ are the conserved charge susceptibilities which are defined as:

$$
\chi_{B, Q, S}^{i, j, k}=\frac{\partial^{i} \partial^{j} \partial^{k} P\left(T, \mu_{B}, \mu_{Q}, \mu_{S}\right) / T^{4}}{\partial\left(\mu_{B} / T\right)^{i} \partial\left(\mu_{Q} / T\right)^{j} \partial\left(\mu_{S} / T\right)^{k}} .
$$

We limit the study only to second order derivatives of the QCD pressure which 
already provides sufficient information to extract the hierarchy of EV sizes in the baryonic, strange, and mesonic sectors of hadronic matter.

Throughout all the following results, it is assumed that the size of non-strange baryons is fixed to the size of the nucleon $v_{\mathrm{B}}=1 \mathrm{fm}^{3}$. This value is found to be in agreement with the microscopical quantum nuclear interactions of nucleons and is also supported by the analysis of LQCD data [1]. The value of $v_{\mathrm{B}}=1$ $\mathrm{fm}^{3}$ corresponds to the proton radius as $R_{\mathrm{p}}=\left(\frac{3}{16 \pi} v_{\mathrm{B}}\right)^{1 / 3} \approx 0.39 \mathrm{fm}$, the value is in agreement with the values suggested by the analysis of NN-scattering phase shift data [151]. In the first step, the sensitivity of the susceptibilities on the strange baryon size is presented. The values of strange baryon sizes are varied as $v_{\mathrm{BS}}=1,1 / 2,1 / 4,1 / 8 \mathrm{fm}^{3}$. The volume of mesons here are initially fixed to $1 / 8$ $\mathrm{fm}^{3}$ as in Ref. [171, 5] and will be varied later.

The resulting second order susceptibilities are presented in Fig. VI.1. As expected, the $B B, S S, B S$ susceptibilities show a strong sensitivity to the size of strange baryons in the temperature range $150<T<250 \mathrm{MeV}$, which can be considered as the transition region between hadrons and quarks. A decrease of the strange baryon size, to $v_{\mathrm{BS}}=1 / 4 \mathrm{fm}^{3}$, allows a reasonable description of the $B B, S S$, and $B S$ susceptibilities.

The susceptibilities which involve the electric charge, however, show much less sensitivity to the strange baryon volume. Since a large fraction of the electric charge is carried by mesons, a change in the meson EV parameter should affect the electric charge susceptibilities. To study the susceptibilities which involve the electric charge, we vary the EV parameters for strange $v_{\mathrm{MS}}$ and non-strange $v_{M}$ mesons while $v_{B}=1 \mathrm{fm}^{3}$ and $v_{\mathrm{BS}}=1 / 4 \mathrm{fm}^{3}$ are fixed as a result of the comparison presented in Fig. VI.1. The results are presented in Fig. VI.2 where four combinations of meson volumes are compared, $v_{\mathrm{M}}=v_{M S}=1 / 8 \mathrm{fm}^{3}$ as in the default version of the CMF, $v_{\mathrm{M}}=v_{M S}=1 / 4 \mathrm{fm}^{3}, v_{\mathrm{M}}=v_{M S}=1 / 2 \mathrm{fm}^{3}$, and $v_{\mathrm{M}}=1 / 2 \mathrm{fm}^{3} v_{\mathrm{MS}}=1 / 4 \mathrm{fm}^{3}$. From these parameterizations the last one, which assumes a larger volume for non-strange mesons, appears to describe the lattice data best.

Consequently, the parameterization with $v_{B}=1 \mathrm{fm}^{3}, v_{\mathrm{BS}}=1 / 4 \mathrm{fm}^{3}, v_{\mathrm{M}}=1 / 2$ $\mathrm{fm}^{3}, v_{\mathrm{MS}}=1 / 4 \mathrm{fm}^{3}$ provides a much improved agreement with LQCD data for the second order $B B, Q Q, S S, B S, Q S$, susceptibilities. Only the $B Q$ susceptibility appears to be unaffected by all EV parameterizations studied above. Since the $B Q$ combination is sensitive to the baryon charge correlations we conjecture that the 


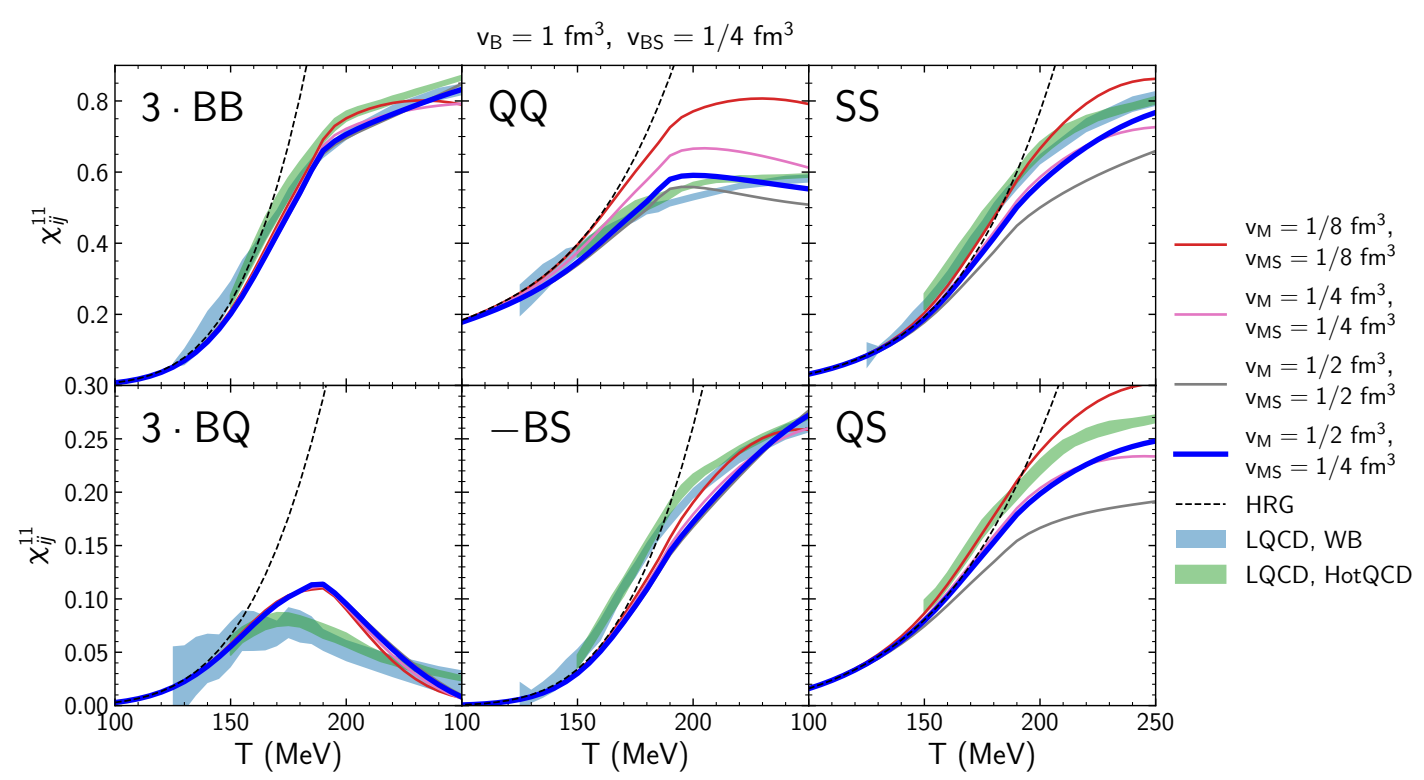

Figure VI.2: The same as Fig. VI.1, but for various excluded volume parameters of mesons $v_{\mathrm{M}}$ and strange mesons $v_{\mathrm{MS}}$. The line which best fits to the lattice data is presented in bold for $v_{\mathrm{M}}=1 / 2 \mathrm{fm}^{3}$ and $v_{\mathrm{MS}}=1 / 4 \mathrm{fm}^{3}$. The EV parameter of strange baryons is fixed to $v_{\mathrm{BS}}=1 / 4 \mathrm{fm}^{3}$ as the best result from Fig. VI.1. The predictions of the CMF model, solid lines, are compared with available results of lattice QCD calculations by the Wuppertal-Budapest collaboration [162] and HotQCD collaborations [230], blue and green colorbands, respectively. The HRG results are also presented by the black dashed lines. The electric charge susceptibilities show a particular strong dependence on the meson EV. Only the baryon-electric charge correlation appears to be insensitive.

$B Q$ susceptibility can be better described by a change of the EV parameters of the $\Delta$ - and $N^{*}$-baryons. This would require one or more additional parameters related to the $\Delta$ and $N^{*}$ repulsive interactions, supporting the scenario of a unique EV parameter for every hadron, which are however, mainly unknown. Such a picture seems reasonable and it would introduce a whole plethora of new parameters which allows for the description of even higher orders of LQCD susceptibilities. 


\section{VI.2 Consequences of the modified excluded volumes}

As discussed above, the introduction of species-dependent repulsive interactions of hadron yields a good description of lattice QCD data, essentially up to an arbitrary order. Such a procedure, however, poses the question what conclusions can be drawn. Instead of trying to understand and justify every parameter, it is more convenient to study the sensitivity of the CMF model predictions for the high density matter on these parameters. In the following, we will discuss how the modified EV parameters change the phase structure of the model and the equation of state for dense nuclear and neutron star matter. Thus, the goal of this section is to explain the consequences of the modified hyperon repulsion on different relevant states of matter: iso-spin symmetric, heavy ion collisions (with strangeness conservation) as well as net strange matter. All these forms of QCD matter can be studied in different experimental and observational scenarios:

1. Iso-spin Symmetric matter: Here one assumes that up and down quarks (as well as protons and neutrons) are equally abundant. This scenario is often studied when one refers to the 'QCD-phase diagram'. In particular we will assume that the strange chemical potential $\mu_{S}=0$ vanishes which can lead to a finite net strangeness.

2. The EOS for Heavy Ion Collisions: This state of matter is close to iso-spin symmetric matter, but obeys an additional constraint of zero net strangeness. This type of matter is created in heavy ion collisions at various beam energies where net strangeness is conserved.

3. Neutron star matter: Neutron stars are cold compact stellar objects which are composed of QCD matter in $\beta$-equilibrium and in local charge neutrality. There at low densities neutrons are much more abundant than protons and strangeness is not conserved, i.e. $\mu_{S}=0$. The densities in the NS interiors surpass several times nuclear saturation density. The description of such matter is essential for the calculations of neutron star properties and stands as a benchmark for QCD phenomenology for a region in the QCD phase diagram which is not accessible by LQCD methods or heavy ion collisions. 


\section{VI.2.1 Phase structure of Iso-spin Symmetric matter}

The interactions in the CMF model provide a reasonable description of the nuclear ground state properties, such as binding energy, compressibility, asymmetry energy, and the slope parameter [5]. The changes of the EV parameters introduced in the previous section do not allow hyperons to appear at below and slightly higher than the nuclear saturation density. As shown in Fig. VI.3 the properties of the nuclear ground state are not affected by the change of EV parameters. The figure shows the energy per baryon $\varepsilon / n_{B}$ at $T=0$ for isospin symmetric matter as function of the order parameter, the chiral condensate $\sigma / \sigma_{0}$. After a density of $n_{B} \approx 0.5$ $\mathrm{fm}^{-3}$ the parameterizations start to deviate for different values of the hyperon EV parameter (at $T=0$ mesons are not excited and the meson Bose condensation is not included in our calculations). The non-monotonic behaviour of the energy per baryon indicates the presence of a phase transition with a metastable state. Note that even though a metastable state with a small energy barrier is created, no absolutely stable state of matter can be generated by the appearance of the hyperons.

The effect of the modified EV parameters on the phase structure is also depicted in figure VI.4. Here we show the net baryon density $n_{B}$ as a function of the baryon chemical potential $\mu_{B}$ for $T=0$ and iso-spin symmetric matter. To better illustrate the position of the first order transition, a Maxwell construction between two coexisting phases was done. For the default version of the CMF, a very weak chiral phase transition appears at $\mu_{B}^{C} \approx 1400 \mathrm{MeV}$, with a critical endpoint at $T_{\mathrm{CP}} \approx 17 \mathrm{MeV}$. As the volume of the hyperons is decreased, this transition gets slightly stronger, i.e., the latent heat and the jump in the density are increased and at the same time the critical chemical potential is increased. It was checked that the value of critical temperature (temperature of the chiral critical point) is not significantly affected by the change of interaction parameters. Thus, the general characteristics of the phase structure, i.e., a critical endpoint at a very low temperature, are not changed.

The standard CMF parameterization, Refs. $[168,171,5]$, yields matter at $T=0$ (assuming $\mu_{S}=0$ ) which is only composed of nucleons and their parity partners. Heavier hadrons as deltas and hyperons are suppressed by the interactions. This is a result of the EV interactions in the CMF model: hadrons are suppressed at higher densities as a result of their repulsive hard core interactions. The quarks become the dominant degrees of freedom in the medium. The degree of 


\section{VI.2. CONSEQUENCES OF THE MODIFIED EXCLUDED VOLUMES}

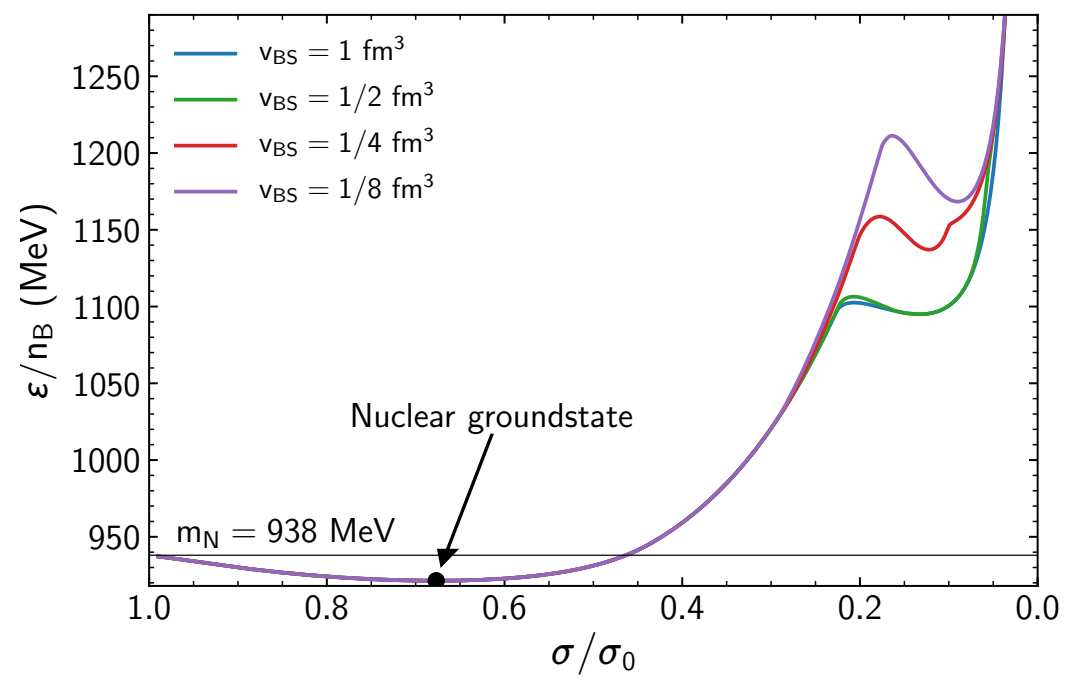

Figure VI.3: Energy per baryon $\varepsilon / n_{B}$ as a function of the chiral condensate $\sigma / \sigma_{0}$ for $T=0$, isospin symmetric nuclear matter. Four different parameterizations of the strange baryon excluded volume $v_{\mathrm{BS}}=1,1 / 2,1 / 4,1 / 8 \mathrm{fm}^{3}$ are presented. While the nuclear ground-state properties are unchanged, a second minimum in the energy per baryon located at smaller values of the chiral condensate, indicating the chiral phase transition, is sensitive to the EV parameterization. This second minimum signals a metastable state of chirally restored matter.

suppression depends on the repulsion coefficient, i.e. the EV parameter. The higher the value of this parameter, the less of the hadrons will be present as the pressure is increased. If the EV coefficient of the strange baryons is smaller than the $\mathrm{EV}$ of the non-strange baryons, then the strange baryons will survive to higher energy densities.

This allows for a distinct type of nuclear matter to emerge prior to the transition to the quark matter. Hyperonic matter thus appears as an additional phase between nuclear and quark matter. Hypermatter is a metastable state which appears as an exotic strange form of matter [232, 233, 234, 235, 236, 237, 238].

Figure VI.5 shows the strangeness per baryon $f_{S}=-\left(n_{S} / n_{B}\right)$ as a function of the baryon density for $T=0$ iso-spin symmetric matter. The limit for $f_{S}$ is 3 as then the matter would be made up completely of strange quarks. A value of $f_{S}=1$ would correspond to $\Lambda$ matter where $1 / 3$ of the baryon charge is carried by the strange quarks. 


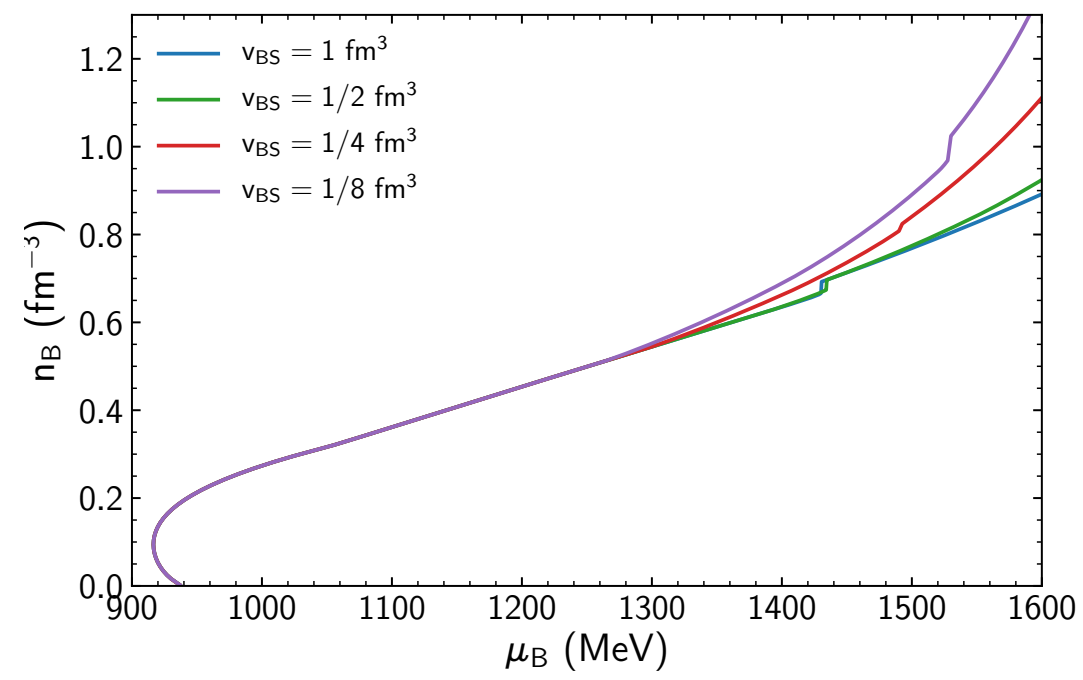

Figure VI.4: Baryon density $n_{B}$ as a function of baryon chemical potential $\mu_{B}$ for $T=0$ isospin symmetric nuclear matter for four different parameterizations of strange baryon excluded volume $v_{\mathrm{BS}}=1,1 / 2,1 / 4,1 / 8 \mathrm{fm}^{3}$. Note that no additional phase transition appears while the chiral transition is shifted to higher values of baryon chemical potential.

The four values of the hyperonic volume are located within the purple band which covers the possible range of $f_{S}$ for $v_{\mathrm{BS}}=1 / 8-1 \mathrm{fm}^{3}$. The dashed lines correspond to the fraction of $f_{S}$ which stems from the hyperons. Since in the scenario with $v_{B S}=1 \mathrm{fm}^{3}$ all strangeness is carried by the $s$-quark, the blue dashed line constantly stays at zero. As the EV of the strange hadrons is decreased, the fraction of hyperonic matter is increased significantly. At the density around $n_{B} \approx 1.5-2 \mathrm{fm}^{-3}$ The hyperons start to be suppressed, this is a result of EV suppression when the free quarks create a significant contribution to the total system pressure. At very high densities, $n_{B} \approx 20 \mathrm{fm}^{-3}$, strangeness fraction $f_{S}$ for all parameterizations coincide, this is where the pure quark matter is produced and all hadrons are completely suppressed. However, a super-rich strange state $f_{S}>1$ is never produced by multistrange baryons and the strangeness fraction increases continuously from 0 to 1 which is also the limit for a free gas with three quark flavors.

As the meta-stable states observed in figure VI.3 appear for systems below the critical temperature of $T_{C P} \approx 17 \mathrm{MeV}$, states of hyperon rich matter may survive here for an extended time. It is questionable whether such a cold and 


\section{VI.2. CONSEQUENCES OF THE MODIFIED EXCLUDED VOLUMES}

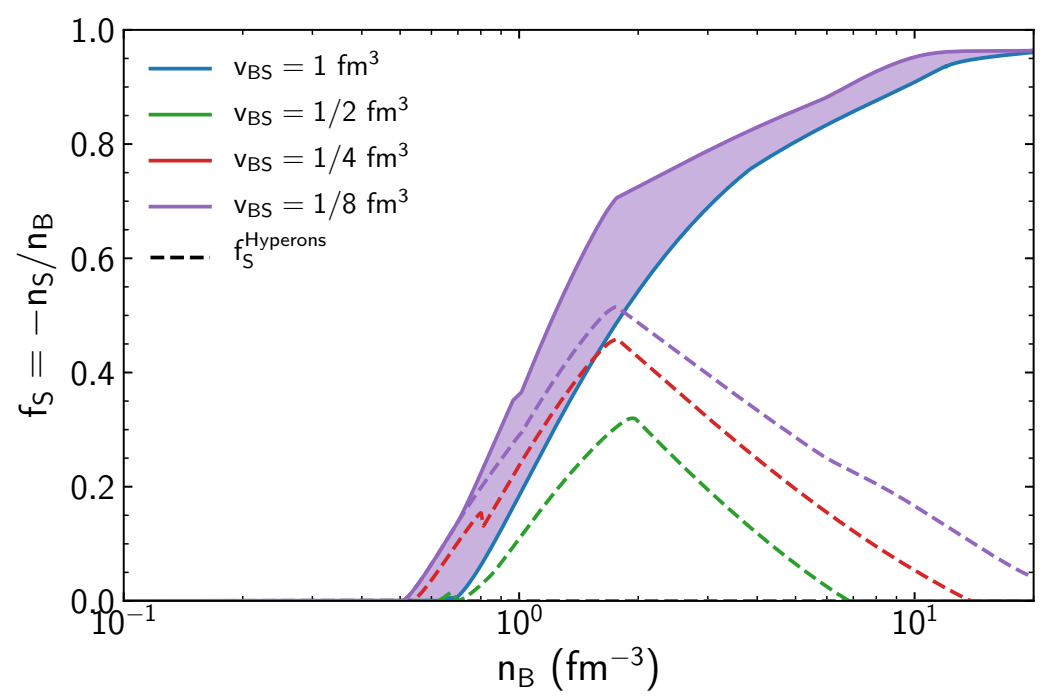

Figure VI.5: Strangeness fraction $f_{S}$ as a function of baryon density $n_{B}$ for $T=0$ isospin symmetric nuclear matter. The band illustrates the range of values due to varying the hyperon EV parameter in range $v_{\mathrm{BS}}=1 / 8-1 \mathrm{fm}^{3}$. The hyperon contribution to strangeness fraction is illustrated by dashed lines. Note, no additional phase transition or a state of bound hyperon matter appear.

dense environment could be created in heavy ion collisions, however in neutron stars and their mergers this scenario appears feasible.

\section{VI.2.2 The EOS for Heavy Ion collisions}

A direct confirmation of the equation of state at high density from heavy ion data would require the space-time simulation of the collision, using the CMF model as input. A first study in this direction was already done for low beam energies using ideal fluid dynamics [239]. However, for heavy ion collisions it is essential to also take into account the non-equilibrium aspects. Early studies extracted an effective nuclear equation of state from the flow data [240]. This method can not be directly be compared to the CMF finite temperature EoS. It is planned to apply the proper treatment to take into account the interactions in relativistic transport through the mean field description at finite temperatures, as shown in $[241,242]$.

This work focuses on thermodynamic properties of the CMF model related to heavy ion collisions. Effects of the different EV parameterizations may be 


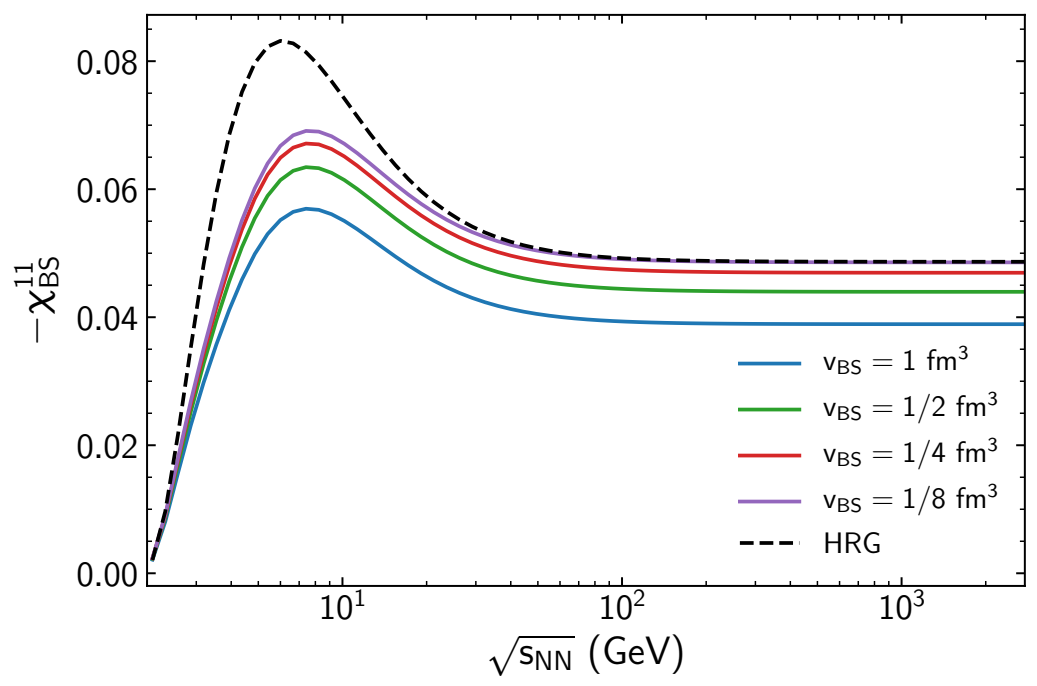

Figure VI.6: Collision energy dependence of baryon-strangeness second order susceptibility $\chi_{\mathrm{BS}}^{11}$ estimated in the CMF model along the chemical freeze-out curve of Ref. [50] for zero net-strangeness isospin-symmetric matter.

observed in the late stages of heavy ion collisions and neutron stars. The change in the repulsive properties leads to different thermodynamic properties of the system at the chemical freeze-out which, potentially, can be measured through the final particle yields. The chemical freeze-out conditions depend on the energy of the nuclear collision, which allows experiments to probe various regions of the QCD phase diagram experimentally. Since the bulk evolution, at any given beam energy, is well characterized by the produced entropy per baryon, the mapping between the collision energy and the expansion path through the phase diagram can be done by the chemical composition of hadrons after the chemical freeze-out $[18$, 19, 20]. For simplicity, we use the so-called freeze-out line for our comparison. Through the measured chemical composition of particles, this line provides a mapping of the collision energy $\sqrt{s_{N N}}$ with temperature $T$ and baryon chemical potential $\mu_{B}$ at the chemical freeze-out. Here the chemical freeze-out curve from Ref. [50] is used.

The strangeness-baryon cross susceptibility $\chi_{B S}^{11}$ is particularly sensitive to the strange hadron EV-parameter: we estimate the values of $\chi_{B S}^{11}$ along the freeze-out line for 4 different values of $v_{\mathrm{BS}}$. Figure VI.6 compares these resulting CMFsusceptibilities $\chi_{B S}^{11}$ with the well known ideal HRG results. As the chemical freeze-out is assumed to occur when matter is quite dilute, moderate effects of 
the EV interactions are observed. (1)

The matter at the studied freeze-out scenario does not produce such significant sensitivity to the EV parameters as the lattice QCD data. In addition, for a meaningful comparison of measured susceptibilities with our model calculation, some elaborate simulations, taking into account the effects of the finite size and lifetime of the system, would be necessary. Thus we conclude that low baryon densities offer for the LQCD data a good benchmark to probe hadronic interactions. These interactions and the related phase structure should be tested with heavy ion collisions in the high baryon density regime, e.g., at FAIR facility. In addition, nuclear astrophysics offers an alternative venue through the study of neutron star properties and binary neutron star mergers with their gravitational wave signals $[205,245,4]$.

\section{VI.2.3 Neutron stars}

Observations of neutron stars provide another way to probe the equation of state of cold and dense nuclear matter and possibly deconfined quark matter. The CMF model, in its default parameterization, gives a satisfactory description of the properties of cold static non-rotating neutron stars. In particular, the mass-radius relation $M(R)$, and the tidal deformability $\Lambda$ [5]. The mass-radius relation is obtained by solving the Tolmann-Oppenheimer-Volkoff (TOV) equation [214, 215], which uses the equation of state as input and provides the density and pressure profiles of the NS. A solution of the TOV equation relates the central density to the NS mass and radius. The densities in the NS's interiors can reach several times nuclear saturation density $n_{0}$. This allows for the formation of quark cores in the interior of the stars [246, 247, 248, 205, 220, 249, 250, 251]. These cases are not yet observed. They could be tested in future by measurements of NS masses and radii, e.g., with the NICER X-ray telescope [252, 253], and by the next generation GW detectors [254, 255]. However, even in the hadronic part of the EOS the chemical composition is not well known. The assumption of $\beta$-equilibrium implies that the matter is dominated by neutrons at densities close to $n_{0}$ and that the

\footnotetext{
(1) A more elaborate scenario for chemical freeze-out which implies two or more separate freezeout points for strange and non-strange particles finds that strange hadrons could freeze-out at 10-15 MeV higher temperatures than the light hadrons at the highest collision energies [243, $244,114]$. A strange freeze-out at these higher temperatures could provide stronger signals of different EV interaction schemes.
} 


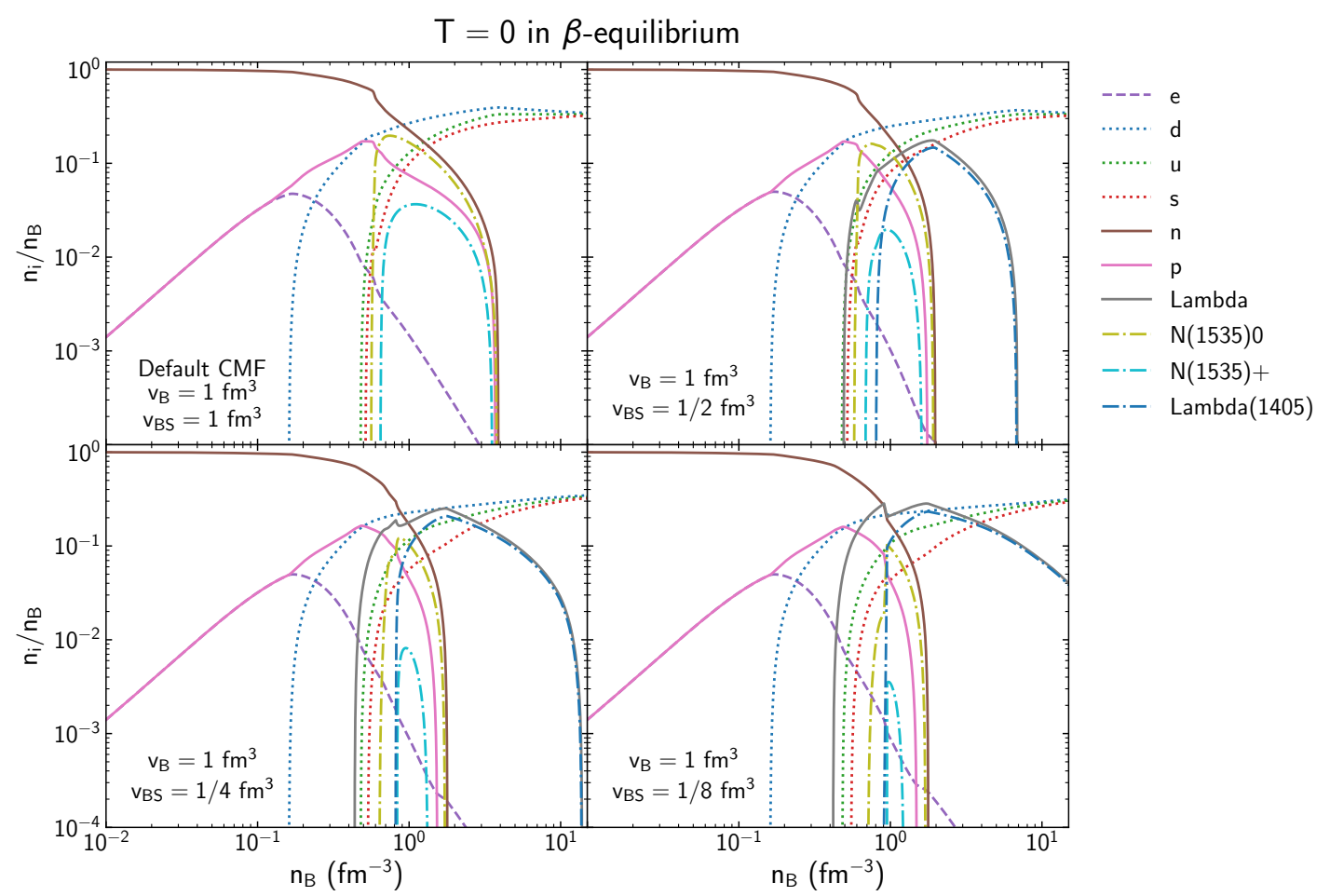

Figure VI.7: Particle composition by the CMF model for $T=0$ matter in $\beta$-equilibrium. Particle number densities $n_{i}$, over the baryon density $n_{B}$ are presented as functions of baryon density, note an additional factor $1 / 3$ for quarks is used. Four plots correspond to four different parameterizations of strange baryon excluded volume $v_{\mathrm{BS}}=1,1 / 2,1 / 4,1 / 8 \mathrm{fm}^{3}$. Electrons and baryons only from the groundstate octet are presented by solid lines, quarks by dotted lines, octet parity partners by dot-dashed lines.

charge of the small admixture of protons is compensated by the same number of electrons ${ }^{(2)}$. With increasing density, heavier hadrons should appear. The implication of the hyperon appearance for the NS properties is actively discussed as "hyperon puzzle", (for a review refer to Ref. [256]), which traces back to the 1960s [257].

Furthermore, in neutron star matter, $d$-quarks are favorable as compared to $u$-quarks or protons due to their opposite electric charge. They are easier to excite than neutrons. Even at $n_{B} \approx 2 n_{0}$ the free quarks make up only to $20 \%$

\footnotetext{
${ }^{(2)}$ Note that we have checked that the inclusion of muons does not alter our results in any significant way.
} 
of the baryonic charge. In the CMF model, the chemical composition of isospin symmetric matter contains no free quarks for $n_{B}<2 n_{0}$, and free quarks make up $20 \%$ of the total baryon number only at $n_{B} \approx 5 n_{0}$.

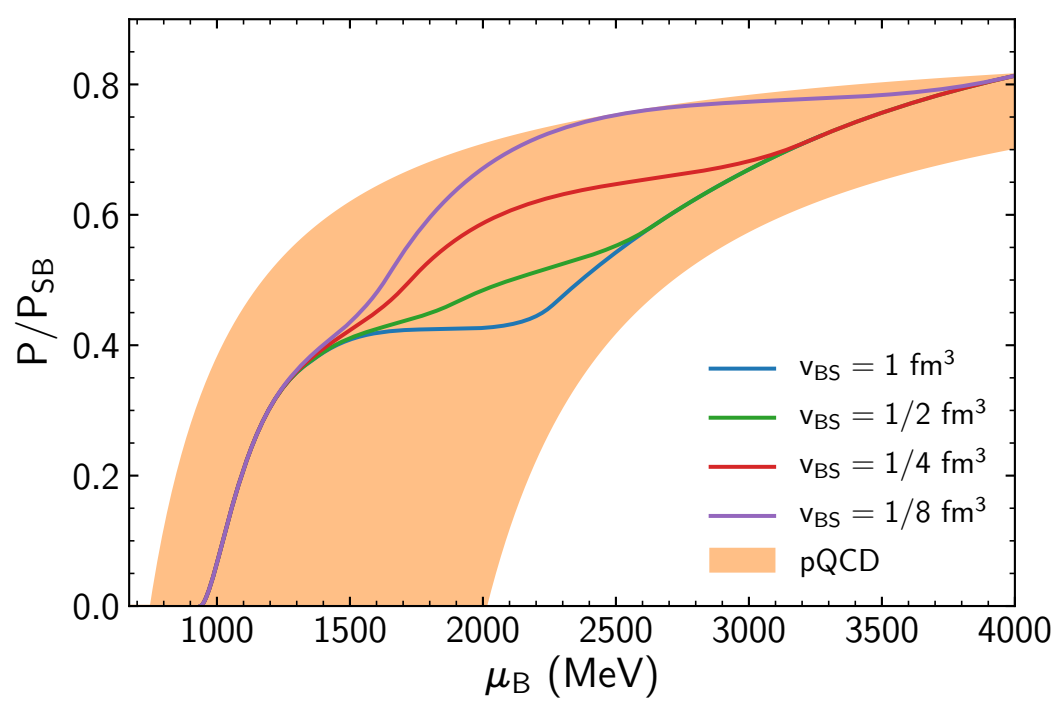

Figure VI.8: The ratio of charge neutral CMF matter pressure $P$ at $T=0$ in $\beta$-equilibrium, $P$, to the Stefan-Boltzmann pressure limit of a massless 3 flavor gas of quarks, $P_{\mathrm{SB}}$. Lines correspond to the four different excluded volume parameters of strange baryons used. The respective particle contents are illustrated in Fig. VI.7. The yellow colorband illustrates parameterization [258] of three-loop pQCD calculations for pressure of cold quark matter in $\beta$-equilibrium [259].

When the hyperon volumes are treated the same as the non-strange baryons, i.e., $v_{\mathrm{SB}}=v_{\mathrm{B}}$, the hyperons in the CMF model are suppressed by both, their higher masses and their EV interactions. Hence, they do not appear in neutron stars [5]. The same is true for any other higher mass baryons which are suppressed at $T=0$ by their repulsive excluded volume interactions. In this case, the hadronic part of the NS is only composed of nucleons and their parity partners. The early appearance of the parity partners, as opposed to, e.g., the Delta baryons which have a smaller vacuum mass, is the parity doubling due to chiral symmetry restoration. The effective masses of heavy parity partners of both $N$ and $\Lambda$ are significantly decreased in chirally restored phase. While the masses of $\Delta \mathrm{s}$ in the current approach remain constant.

This scenario can significantly change as the EV-parameter of the hyperons is 
reduced: the chemical composition of neutron star matter is shown in Fig. VI.7 as a function of the baryon density. The non-strange baryon EV parameter is fixed at $v_{\mathrm{B}}=1 \mathrm{fm}^{3}$, while the $\mathrm{EV}$ parameter of strange baryons is varied, $v_{\mathrm{BS}}=1,1 / 2,1 / 4,1 / 8 \mathrm{fm}^{3}$. Decreasing the hyperon repulsion allows $\Lambda$-baryons and their parity partners $\Lambda(1405)$ to populate NS matter, while heavier hyperons are suppressed due to their higher mass. The threshold for the appearance of $\Lambda$ is $n_{B} \approx 0.3-0.4 \mathrm{fm}^{-3}$ (for all values of $v_{\mathrm{BS}}$ except the largest). This is clearly below the density of the chiral phase transition in the CMF model. The location of the chiral transition is sensitive to $v_{\mathrm{BS}}$ as well: for $v_{\mathrm{BS}}=1 \mathrm{fm}^{3}$ and $1 / 2 \mathrm{fm}^{3}$ the transition is located at $n_{B} \approx 0.6 \mathrm{fm}^{-3}$. For $v_{\mathrm{BS}}=1 / 4 \mathrm{fm}^{3}$ it is shifted to higher density, $n_{B} \approx 0.8 \mathrm{fm}^{-3}$. The transition is located at $n_{B} \approx 1 \mathrm{fm}^{-3}$ for $v_{\mathrm{BS}}=1 / 8$ $\mathrm{fm}^{3}$. At the chiral transition, the parity partner mass drops to the $\Lambda$-mass. Hence, $\Lambda(1405)$ contributes to the strangeness fraction similarly to the octet $\Lambda$-hyperon.

The reduced $v_{\mathrm{BS}}$-repulsion in strange baryon sector yields a significant hyperon fraction of the total baryon density. If the repulsion among the strange particles is 8 times smaller than among non-strange, as illustrated in Fig. VI.7 which shows that for $v_{\mathrm{BS}}=1 / 8 \mathrm{fm}^{3}$, the hyperons can survive up to extreme densities of $10 \mathrm{fm}^{-3}$ and even more. At these densities quarks are the dominant degrees of freedom. However this type of matter is distinct from the quark matter due to the small admixture of the strange hadrons.

The appearance of the additional hyperon degrees of freedom leads to a softening of the NS-matter EOS. This inevitably changes the properties of neutron stars. To illustrate the change of the EOS due to $v_{\mathrm{BS}}$, Fig. VI.8 shows the pressure $P$ for the CMF calculations as a function of the baryon chemical potential $\mu_{B}$ as compared to the results of pQCD calculations [259]. The additional degrees of freedom at a given chemical potential yield additional pressure. For the values $v_{\mathrm{BS}}=1 / 4,1 / 8 \mathrm{fm}^{3}$ a significant increase in $P / P_{\mathrm{SB}}$ is observed. This is a result of the sudden appearance and subsequent suppression of hyperons in the EOS. For $v_{\mathrm{BS}}=1 / 8 \mathrm{fm}^{3}$ the increase reaches the borders of the pQCD bands of confidence suggesting that it can be considered as an absolute lower bound for $v_{\mathrm{BS}}$. However, all parameterizations fit within the pQCD band and merge into one line at the region of chemical potential $\mu_{B}>3500 \mathrm{MeV}$ where the pQCD bands become narrow, there the baryon densities are extreme with $n_{B}>20 \mathrm{fm}^{3}$. At these values of $\mu_{B}$, as predicted by the CMF model, the matter is composed of free quarks only, without the admixture of hadrons. At the lower values of the chemical potential 
the pQCD bands permit various scenarios of hadron-quark interactions as shown by the CMF results.

These differences of the EOS due to the possible variation of $v_{B S}$ change the properties of neutron stars: Fig. VI.9 depicts the mass-radius relations as calculated from the Tollman-Oppenheimer-Volkov equation ${ }^{(3)}$, for the EOS parameterizations discussed above. The additional degrees of freedom results in a softening of the EOS. This decreases the maximum mass of the NS families by $5 \%$, from $M^{\max } \approx 2.15 M_{\odot}$ to $M^{\max } \approx 2.05 M_{\odot}$. The differences appear only in the highest mass region because the hyperons modify the EOS. These high densities can be reached only in the most massive stars. Mergers of neutron stars yield much higher densities and high temperatures. Therefore, a study of the effects of the hyperonic repulsion in simulations of neutron star mergers is needed. Another worthwhile study could be the effect on neutron star cooling: an early study of the CMF model in the context of parity doubling showed that the cooling curve can be reasonably well described within this model. There, certain assumptions on the role of the parity partners are made [261].

${ }^{(3)}$ The numerical solutions were obtained using the TOV solver of [260]. 


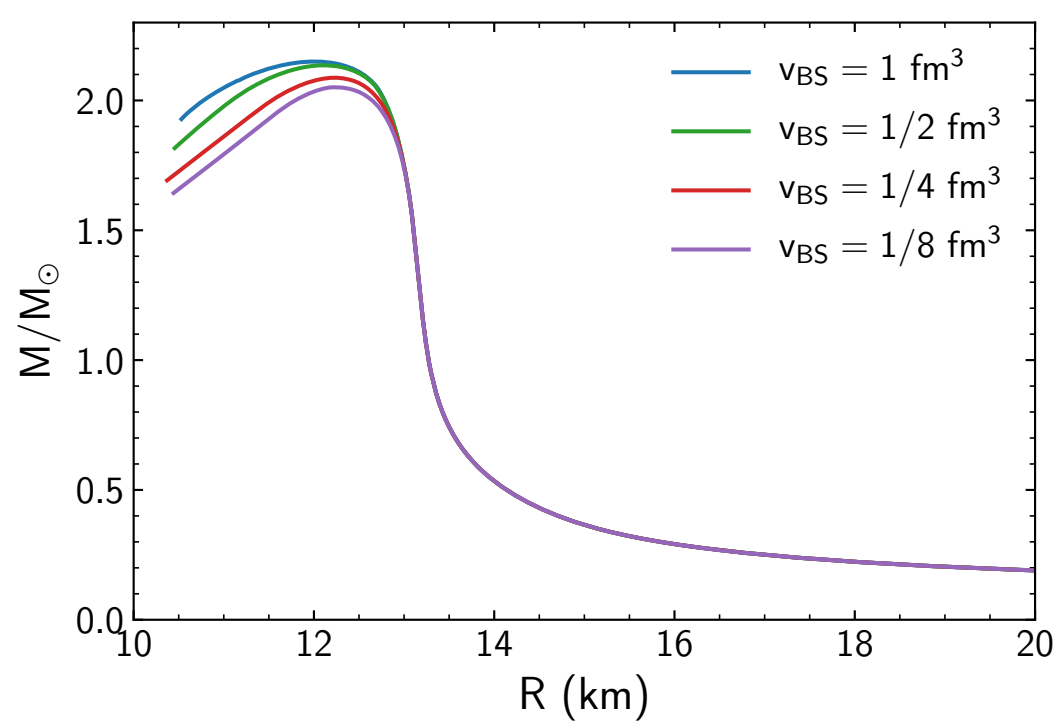

Figure VI.9: The CMF mass-radius diagrams for four different excluded volume parameters of strange baryons. The respective particle content is illustrated in Fig. VI.7. Note that the appearance of numerous strange baryons only slightly changes the mass-radius diagram, substantially affecting only the unstable branch of the solutions. 
106 VI.2. CONSEQUENCES OF THE MODIFIED EXCLUDED VOLUMES 


\section{VII}

\section{Summary and Outlook}

The results presented in this thesis are related to the following three topics: the dynamics of the QCD matter in heavy ion collisions, QCD matter in compact stars, and the phase structure of the QCD matter. Thus, this thesis is devoted to the properties of the QCD matter under different conditions. The first half of the thesis is dedicated to the QCD matter in the hadronic phase, and how it reveals in the late evolution of heavy ion collisions. In the second part, a phenomenological approach for QCD matter at a wide range of temperatures and densities, the CMF model, is presented. The CMF model includes many aspects of QCD phenomenology, which makes the model applicable simultaneously to heavy ion collisions, neutron stars, and analysis of lattice QCD data.

As a first step, the hadronic observables like the $K / \pi$ ratio and scaled variance of negative charged particles are studied in hadronic models, the microscopic transport UrQMD model, and the statistical thermal model. A non-monotonic behavior of the studied observables is often used as the signature of a phase change during heavy ion collisions. However, the experimentally measured values should be considered with care, since dynamical contributions can significantly affect the measured values. The effects of charge conservation, finite detector acceptance, and centrality selection are estimated. A comparison with available experimental data from NA61/SHINE and NA49 collaborations is done for $K^{+} / \pi^{+}$ratio and scaled variance of negatively charged particles $\omega^{-}$measured for $\mathrm{p}+\mathrm{p}$ and $\mathrm{A}+\mathrm{A}$ collisions at SPS energy range. The used models are purely hadronic and do not include transitions to other QCD phases, however, the predictions of the thermal 
model and UrQMD are strongly sensitive to the centrality selection and detector acceptance. It is found that the non-monotonic behavior of $\omega^{-}$as a function of the colliding system size is well reproduced by the UrQMD model, it is discovered that the non-monotonicity is attributed to the centrality selection. The main result from the study is that a centrality selection procedure in $\mathrm{p}+\mathrm{p}$ reactions should be incorporated to remove dynamical effects that significantly modify the value of event-by-event particle number fluctuations.

A novel method to extract the temperature of kinetic freeze-out based on the measured yields of the short-lived resonances such as $\rho^{0}$ and $\mathrm{K}^{* 0}$ was presented. The suggested method assumes that the system created in heavy ion collision evolves between the chemical and kinetic freeze-outs as expanding hadron resonance gas in partial chemical equilibrium. This method, contrary to conventional blast-wave fits, does not require additional input such as the flow velocity profile and the freeze-out hypersurface. In this sense, the PCE-HRG approach to extracting $T_{\text {kin }}$ is advantageous to the commonly adopted fits to the $p_{T}$ spectra. The ALICE data on $\mathrm{Pb}-\mathrm{Pb}$ collisions at the LHC yields was analysed with the PCE-HRG approach, the analysis yields a moderate multiplicity dependence of $T_{\mathrm{ch}}$ whereas the kinetic freeze-out temperature drops from $T_{\text {kin }} \simeq T_{\text {ch }} \simeq 155 \mathrm{MeV}$ in peripheral collisions to $T_{\text {kin }} \simeq 110 \mathrm{MeV}$ in $0-20 \%$ most central collisions. This result is in qualitative agreement with prior studies employing the blast-wave model fits. The presented framework can be applied to lower collision energies and used to analyze other sensitive probes of freeze-out dynamics, such as fluctuations and correlations of identified hadron numbers.

These results indicate that the dynamics of the simplest phase of QCD, the ideal Hadron Resonance Gas, in heavy ion collisions, can already produce nontrivial observations. However, description of QCD matter requires efforts that go much further than the non-interacting gas of hadrons. An analysis of lattice QCD data hints at the importance of repulsive interactions among hadrons. The strength of hadron repulsive interactions between nucleons/baryons, with quantum effects taken into account, can be estimated by the Beth-Uhlenbeck (BU) approach. In this thesis, the second virial coefficient, the excluded volume (EV) parameter, is calculated within the BU formalism. Here, the hard-core two-particle scattering potential with radii $r_{c}=0.25-0.3 \mathrm{fm}$ between nucleons is assumed. It is found that quantum effects cause the excluded volume parameter to be temperaturedependent, contrary to classical van der Waals approach. The calculated BU 
second virial coefficient is in a fair agreement with S-matrix calculations based on empirical phase shifts of nucleon scatterings. The calculated values of EV parameter are used to describe the repulsive interactions among baryons in the EV-HRG description of QCD matter. The predictions for net baryon number susceptibilities are compared to lattice QCD calculations. It is found that this modified Beth-Uhlenbeck approach describes fairly well the deviations of the lattice QCD data from the ideal HRG model at $T \lesssim 160 \mathrm{MeV}$. The excluded volume HRG model with the temperature dependent baryonic eigenvolume, on the other hand, extends the agreement with the lattice data for baryon number susceptibilities even to temperatures beyond $160 \mathrm{MeV}$. It is found that the excluded volume HRG model with a constant effective baryonic "excluded-volume" parameter $v_{B B}=1 \mathrm{fm}^{3}$ provides a simple yet efficient description of the net effect of the repulsive and attractive baryon-baryon interactions on the hadronic equation of state in the crossover temperature region.

The remaining part of the present thesis presents an attempt to describe the QCD matter in a unified approach appropriate at essentially all temperatures and densities relevant for both heavy ion collisions and neutron star matter. This was done utilizing the improved CMF model which, besides many other features, describes lattice QCD data on thermodynamics of QCD matter at $\mu_{B}=0$. The CMF model allows for a simultaneous description of many nuclear (astro-) physical data, consistent with astrophysical observations as well as heavy ion collisions and of compact stars. The CMF model is used to explore the phase diagram of QCD matter at a wide range of $T$ and $\mu_{B}$. Three critical regions are found, which are connected to the nuclear liquid-vapor phase transition, to the chiral symmetry restoration, and to the quark matter. The model predicts two critical points, first one associated with nuclear liquid-vapor phase transition, and one from chiral symmetry restoration. The transition to quark matter is always a smooth crossover. The region of phase diagram accessible to experiments of high energy heavy-ions collisions is dominated by remnants of the nuclear liquid-vapor phase transition. Other critical regions may be probed by the neutron star structure and in binary neutron star mergers. Different scenarios of hard-core interactions in the CMF model are studied. The sensitivity of the available lattice QCD data for the second order susceptibilities $\chi_{i j}^{11}$ of baryon, electric, and strangeness charges, $B$, $Q$, and $S$, to the values of hadron excluded volume parameters is examined. It is found that $\chi_{i j}^{11}$ are especially sensitive to the short-range repulsive interactions of 
hyperons. Decreasing the hyperons size, as compared to the size of the non-strange baryons, does improve significantly the agreement of the CMF model results with the Lattice QCD data. The electric charge-dependent susceptibilities are sensitive to the excluded volume of mesons. The predicted by CMF properties of neutron stars, like the mass-radius relation, the chemical composition of the stars and the tidal deformabilities are in good agreement with recent experimental observations. The applicability of the CMF model to such a wide range of strongly interacting systems is impressive. In this thesis, for the first time, a QCD-motivated EoS is presented which precisely describes the thermodynamic observables for the whole QCD phase diagram.

Overall, the results of the CMF model presented in this thesis pave a road to bringing together heavy ion collisions with physics of neutron stars. QCD matter described by the CMF model is, with slightly different chemical composition, the same matter as the hot matter created in heavy ion collisions and as the cold compressed matter contained in neutron star interiors and their mergers. In the new era of gravitational wave astronomy, detections of neutron star mergers provide a new way to study hot QCD matter with densities unavailable in heavy ion collisions. The CMF model provides a rich spectrum of possibilities to compare heavy ion collisions with neutron star mergers. The next and the most natural way will be to utilize the equation of state of the CMF model in both hydrodynamic simulations of relativistic nuclear collisions and general-relativistic mergers of compact stars. 


\section{Bibliography}

[1] Volodymyr Vovchenko, Anton Motornenko, Mark I. Gorenstein, and Horst Stoecker. In: Phys. Rev. C97.3 (2018), p. 035202. DoI: 10.1103/PhysRevC. 97.035202. arXiv: 1710.00693 [nucl-th].

[2] Anton Motornenko, Volodymyr Vovchenko, Jan Steinheimer, Stefan Schramm, and Horst Stoecker. In: Nucl. Phys. A982 (2019), pp. 891-894. DOI: 10. 1016/j.nuclphysa.2018.11.028. arXiv: 1809.02000 [hep-ph].

[3] A. Motornenko, V. V. Begun, V. Vovchenko, M. I. Gorenstein, and H. Stoecker. In: Phys. Rev. C 99.3 (2019), p. 034909. Dor: 10.1103/PhysRevC. 99.034909. arXiv: 1811.10645 [nucl-th].

[4] Matthias Hanauske, Jan Steinheimer, Anton Motornenko, Volodymyr Vovchenko, Luke Bovard, Elias R. Most, L. Jens Papenfort, Stefan Schramm, and Horst Stoecker. In: Particles 2.1 (2019), pp. 44-56. DOI: 10.3390/ particles2010004.

[5] Anton Motornenko, Jan Steinheimer, Volodymyr Vovchenko, Stefan Schramm, and Horst Stoecker. In: Phys. Rev. C 101.3 (2020), p. 034904. DOI: 10. 1103/PhysRevC.101.034904. arXiv: 1905.00866 [hep-ph].

[6] Anton Motornenko, Jan Steinheimer, Volodymyr Vovchenko, Stefan Schramm, and Horst Stoecker. In: PoS CORFU2018 (2019). Ed. by Konstantinos Anagnostopoulos et al., p. 150. DOI: 10.22323/1.347.0150. arXiv: 1907.05921 [hep-ph].

[7] Anton Motornenko, Volodymyr Vovchenko, Carsten Greiner, and Horst Stoecker. In: Phys. Rev. C 102.2 (2020), p. 024909. DOI: 10.1103/PhysRevC. 102.024909. arXiv: 1908.11730 [hep-ph]. 
[8] Anton Motornenko, Jan Steinheimer, Volodymyr Vovchenko, Stefan Schramm, and Horst Stoecker. In: Nucl. Phys. A 1005 (2021). Ed. by Feng Liu, Enke Wang, Xin-Nian Wang, Nu Xu, and Ben-Wei Zhang, p. 121836. DOI: 10.1016/j.nuclphysa.2020.121836. arXiv: 2002.01217 [hep-ph].

[9] Anton Motornenko, Somenath Pal, Abhijit Bhattacharyya, Jan Steinheimer, and Horst Stoecker. In: (Sept. 2020). arXiv: 2009.10848 [hep-ph].

[10] B. P. Abbott et al. In: Phys. Rev. Lett. 119.16 (2017), p. 161101. DoI: 10.1103/PhysRevLett.119.161101. arXiv: 1710.05832 [gr-qc].

[11] Enrico Fermi. In: Prog. Theor. Phys. 5 (1950), pp. 570-583. DoI: 10.1143/ PTP.5.570.

[12] L. D. Landau. In: Izv. Akad. Nauk Ser. Fiz. 17 (1953), pp. 51-64.

[13] Krishna Rajagopal. In: Nucl. Phys. A661 (1999), pp. 150-161. DoI: 10. 1016/S0375-9474(99)85017-9. arXiv: hep-ph/9908360 [hep-ph].

[14] Mark G. Alford. In: Nucl. Phys. Proc. Suppl. 117 (2003). [,65(2002)], pp. 65-82. DOI: 10.1016/S0920-5632(03)01411-7. arXiv: hep-ph/0209287 [hep-ph].

[15] Michael Buballa. In: Phys. Rept. 407 (2005), pp. 205-376. DOI: 10.1016/j. physrep.2004.11.004. arXiv: hep-ph/0402234 [hep-ph].

[16] Thomas Schäfer. "Quark matter". In: [,185(2003)]. 2003, pp. 185-233. arXiv: hep-ph/0304281 [hep-ph] .

[17] Kenji Fukushima and Tetsuo Hatsuda. In: Rept. Prog. Phys. 74 (2011), p. 014001. DOI: 10.1088/0034-4885/74/1/014001. arXiv: 1005.4814 [hep-ph].

[18] J. Cleymans and H. Satz. In: Z. Phys. C 57 (1993), pp. 135-148. DoI: 10.1007/BF01555746. arXiv: hep-ph/9207204.

[19] P. Braun-Munzinger and J. Stachel. In: Nucl. Phys. A 606 (1996), pp. 320328. DOI: 10.1016/0375-9474(96)00198-4. arXiv: nucl-th/9606017.

[20] F. Becattini, J. Cleymans, A. Keranen, E Suhonen, and K. Redlich. In: Phys. Rev. C64 (2001), p. 024901. DOI: 10.1103/PhysRevC.64.024901. arXiv: hep-ph/0002267 [hep-ph]. 
[21] Anton Andronic, Peter Braun-Munzinger, Krzysztof Redlich, and Johanna Stachel. In: Nature 561.7723 (2018), pp. 321-330. DOI: 10.1038/s41586018-0491-6. arXiv: 1710.09425 [nucl-th].

[22] J. Cleymans, H. Oeschler, K. Redlich, and S. Wheaton. In: Phys. Rev. C 73 (2006), p. 034905. DOI: 10.1103/PhysRevC.73.034905. arXiv: hep$\mathrm{ph} / 0511094$.

[23] A. Andronic, P. Braun-Munzinger, and J. Stachel. In: Nucl. Phys. A772 (2006), pp. 167-199. DOI: 10.1016/j.nuclphysa.2006.03.012. arXiv: nuclth/0511071 [nucl-th].

[24] A. Bazavov et al. In: Phys. Rev. D90 (2014), p. 094503. DoI: 10.1103/ PhysRevD.90.094503. arXiv: 1407.6387 [hep-lat].

[25] Szabolcs Borsanyi, Zoltan Fodor, Christian Hoelbling, Sandor D Katz, Stefan Krieg, Claudia Ratti, and Kalman K. Szabo. In: JHEP 09 (2010), p. 073. DOI: 10.1007/JHEP09(2010)073. arXiv: 1005.3508 [hep-lat].

[26] A. Bazavov et al. In: Phys. Rev. D 85 (2012), p. 054503. DoI: 10.1103/ PhysRevD.85.054503. arXiv: 1111.1710 [hep-lat].

[27] Adam Miklos Halasz, A.D. Jackson, R.E. Shrock, Misha A. Stephanov, and J.J.M. Verbaarschot. In: Phys. Rev. D 58 (1998), p. 096007. DoI: 10.1103/PhysRevD.58.096007. arXiv: hep-ph/9804290.

[28] DJ Gross. In: Proceedings of the National Academy of Sciences of the United States of America 93.25 (Dec. 1996), pp. 14256-14259. ISSN: 0027-8424. DOI: 10.1073/pnas.93.25.14256. URL: https://europepmc.org/articles/ PMC34470.

[29] Chen Ning Yang. In: Proceedings of the American Philosophical Society 140.3 (1996), pp. 267-288. ISSN: 0003049X. URL: http://www.jstor.org/ stable/987310.

[30] Albert Einstein. In: Annalen Phys. 17 (1905), pp. 891-921. DOI: 10.1002/ andp.200590006.

[31] Emmy Noether. In: Gott. Nachr. 1918 (1918), pp. 235-257. DOI: 10.1080/ 00411457108231446 . arXiv: physics/0503066.

[32] Chen-Ning Yang and Robert L. Mills. In: Phys. Rev. 96 (1954). Ed. by Jong-Ping Hsu and D. Fine, pp. 191-195. DoI: 10.1103/PhysRev.96.191. 
[33] T. Nakano and K. Nishijima. In: Prog. Theor. Phys. 10 (1953), pp. 581-582. DOI: $10.1143 /$ PTP.10.581.

[34] Kazuhiko Nishijima. In: Prog. Theor. Phys. 13.3 (1955), pp. 285-304. DoI: 10.1143/PTP.13.285.

[35] M. Gell-Mann. In: Nuovo Cim. 4.S2 (1956), pp. 848-866. DOI: 10.1007/ BF02748000.

[36] N. Bogolubov, B. Struminsky, and A. Tavkhelidze. In: JINR Preprint D-1968 (1965).

[37] O. W. Greenberg. In: Phys. Rev. Lett. 13 (1964), pp. 598-602. DoI: 10. 1103/PhysRevLett.13.598.

[38] M. Y. Han and Yoichiro Nambu. In: Phys. Rev. 139 (1965). Ed. by T. Eguchi, B1006-B1010. DoI: 10.1103/PhysRev.139.B1006.

[39] John Ellis, Mary K. Gaillard, and Dimitri V. Nanopoulos. "A Historical Profile of the Higgs Boson". In: The standard theory of particle physics: Essays to celebrate CERN's 60th anniversary. Ed. by Luciano Maiani and Luigi Rolandi. 2016. DOI: 10.1142/9789814733519_0014. arXiv: 1504.07217 [hep-ph].

[40] Abdus Salam and John Clive Ward. In: Nuovo Cim. 11 (1959), pp. 568-577. DOI: $10.1007 / \mathrm{BF} 02726525$.

[41] Sheldon L. Glashow. In: Nucl. Phys. 10 (1959), pp. 107-117. DoI: 10.1016/ 0029-5582(59)90196-8.

[42] Steven Weinberg. In: Phys. Rev. Lett. 19 (1967), pp. 1264-1266. DOI: 10.1103/PhysRevLett.19.1264.

[43] F. Englert and R. Brout. In: Phys. Rev. Lett. 13 (1964). Ed. by J. C. Taylor, pp. 321-323. DOI: 10.1103/PhysRevLett.13.321.

[44] Peter W. Higgs. In: Phys. Rev. Lett. 13 (1964). Ed. by J. C. Taylor, pp. 508509. DOI: 10.1103/PhysRevLett.13.508.

[45] Gert Aarts, Chris Allton, Davide De Boni, and Benjamin Jäger. In: (2018). arXiv: 1812.07393 [hep-lat].

[46] F. Gursey. In: Nuovo Cim. 16 (1960), pp. 230-240. DOI: 10.1007/BF02860276.

[47] Murray Gell-Mann and M Levy. In: Nuovo Cim. 16 (1960), p. 705. DOI: 10.1007/BF02859738. 
[48] Gert Aarts, Chris Allton, Davide De Boni, Simon Hands, Benjamin Jäger, Chrisanthi Praki, and Jon-Ivar Skullerud. In: JHEP 06 (2017), p. 034. DOI: 10.1007/JHEP06(2017)034. arXiv: 1703.09246 [hep-lat].

[49] F. Becattini and Ulrich W. Heinz. In: Z. Phys. C 76 (1997). [Erratum: Z.Phys.C 76, 578 (1997)], pp. 269-286. DOI: 10.1007/s002880050551. arXiv: hep-ph/9702274.

[50] V. Vovchenko, V.V. Begun, and M.I. Gorenstein. In: Phys. Rev. C 93.6 (2016), p. 064906. DOI: 10.1103/PhysRevC.93.064906. arXiv: 1512.08025 [nucl-th].

[51] Sandeep Chatterjee, Ajay Kumar Dash, and Bedangadas Mohanty. In: $J$. Phys. G 44.10 (2017), p. 105106. DOI: 10.1088/1361-6471/aa8857. arXiv: 1608.00643 [nucl-th].

[52] V.V. Begun, V. Vovchenko, M.I. Gorenstein, and H. Stoecker. In: Phys. Rev. C 98.5 (2018), p. 054909. DoI: 10.1103/PhysRevC.98.054909. arXiv: 1805.01901 [nucl-th].

[53] V.V. Begun, Mark I. Gorenstein, and O.S. Zozulya. In: Phys. Rev. C 72 (2005), p. 014902. DOI: 10.1103/PhysRevC.72.014902. arXiv: nuclth/0411003.

[54] Volodymyr Vovchenko and Horst Stoecker. In: Comput. Phys. Commun. 244 (2019), p. 295. DOI: 10.1016/j.cpc.2019.06.024. arXiv: 1901.05249 [nucl-th].

[55] Jean Letessier and Johann Rafelski. In: Phys. Rev. C 59 (1999), pp. 947954. DOI: 10.1103/PhysRevC.59.947. arXiv: hep-ph/9806386.

[56] Jean Letessier and Johann Rafelski. In: Eur. Phys. J. A 35 (2008), pp. 221242. DOI: 10.1140/epja/i2007-10546-7. arXiv: nucl-th/0504028.

[57] Johann Rafelski. In: Eur. Phys. J. A 51.9 (2015), p. 114. DOI: 10.1140/ epja/i2015-15114-0. arXiv: 1508.03260 [nucl-th].

[58] Volodymyr Vovchenko, Mark I. Gorenstein, and Horst Stoecker. In: Phys. Rev. C 98.6 (2018), p. 064909. DoI: 10.1103/PhysRevC.98.064909. arXiv: 1805.01402 [nucl-th].

[59] Jean Letessier, Ahmed Tounsi, Ulrich W. Heinz, Josef Sollfrank, and Johann Rafelski. In: Phys. Rev. D 51 (1995), pp. 3408-3435. DoI: 10.1103/ PhysRevD.51.3408. arXiv: hep-ph/9212210. 
[60] P. Castorina, S. Plumari, and H. Satz. In: Int. J. Mod. Phys. E 25.08 (2016), p. 1650058. DOI: 10.1142/S0218301316500580. arXiv: 1603.06529 [hep-ph].

[61] Anton Motornenko, Katarzyna Grebieszkow, Elena Bratkovskaya, Mark I. Gorenstein, Marcus Bleicher, and Klaus Werner. In: J. Phys. G 45.11 (2018), p. 115104. DOI: 10.1088/1361-6471/aae149. arXiv: 1711.07789 [nucl-th].

[62] S.A. Bass et al. In: Prog. Part. Nucl. Phys. 41 (1998), pp. 255-369. DoI: 10.1016/S0146-6410(98)00058-1. arXiv: nucl-th/9803035.

[63] M. Bleicher et al. In: J. Phys. G 25 (1999), pp. 1859-1896. DOI: 10.1088/ 0954-3899/25/9/308. arXiv: hep-ph/9909407.

[64] Andrey Seryakov. In: (2018). URL: https:// edms.cern.ch / document/ 1867336/1.

[65] C. Alt et al. In: Phys. Rev. C 78 (2008), p. 034914. Dor: 10.1103/PhysRevC. 78.034914. arXiv: 0712.3216 [nucl-ex].

[66] V.P. Konchakovski, B. Lungwitz, M.I. Gorenstein, and E.L. Bratkovskaya. In: Phys. Rev. C 78 (2008), p. 024906. DOI: 10.1103/PhysRevC.78.024906. arXiv: 0712.2044 [nucl-th].

[67] M.I. Gorenstein and M. Gazdzicki. In: Phys. Rev. C 84 (2011), p. 014904. DOI: 10.1103/PhysRevC.84.014904. arXiv: 1101.4865 [nucl-th].

[68] A. Bialas, M. Bleszynski, and W. Czyz. In: Nucl. Phys. B 111 (1976), pp. 461-476. DOI: 10.1016/0550-3213(76)90329-1.

[69] A. Aduszkiewicz et al. In: Eur. Phys. J. C 77.10 (2017), p. 671. DOI: 10.1140/epjc/s10052-017-5260-4. arXiv: 1705.02467 [nucl-ex].

[70] Antoni Aduszkiewicz. In: Nucl. Phys. A 967 (2017). Ed. by Ulrich Heinz, Olga Evdokimov, and Peter Jacobs, pp. 35-42. DOI: 10.1016/j.nuclphysa. 2017.04.046. arXiv: 1704.08071 [hep-ex].

[71] C. Alt et al. In: Phys. Rev. C 78 (2008), p. 034918. Dor: 10.1103/PhysRevC. 78.034918. arXiv: 0804.3770 [nucl-ex].

[72] E.L. Bratkovskaya, M. Bleicher, M. Reiter, S. Soff, Horst Stoecker, M. van Leeuwen, S.A. Bass, and W. Cassing. In: Phys. Rev. C 69 (2004), p. 054907. DOI: 10.1103/PhysRevC.69.054907. arXiv: nucl-th/0402026. 
[73] A. Palmese, W. Cassing, E. Seifert, T. Steinert, P. Moreau, and E.L. Bratkovskaya. In: Phys. Rev. C 94.4 (2016), p. 044912. DoI: 10.1103/ PhysRevC.94.044912. arXiv: 1607.04073 [nucl-th].

[74] Marek Gazdzicki. In: (2018).

[75] Francesco Becattini, Marcus Bleicher, Thorsten Kollegger, Tim Schuster, Jan Steinheimer, and Reinhard Stock. In: Phys. Rev. Lett. 111 (2013), p. 082302. DOI: 10.1103/PhysRevLett.111.082302. arXiv: 1212.2431 [nucl-th].

[76] Michal Petrán, Jean Letessier, Vojtěch Petráček, and Johann Rafelski. In: Phys. Rev. C88.3 (2013), p. 034907. DoI: 10.1103/PhysRevC.88.034907. arXiv: 1303.2098 [hep-ph] .

[77] L. Adamczyk et al. In: Phys. Rev. C96.4 (2017), p. 044904. DoI: 10.1103/ PhysRevC.96.044904. arXiv: 1701.07065 [nucl-ex].

[78] M. M. Aggarwal et al. In: Phys. Rev. C84 (2011), p. 034909. DoI: 10.1103/ PhysRevC.84.034909. arXiv: 1006.1961 [nucl-ex].

[79] T. Anticic et al. In: Phys. Rev. C84 (2011), p. 064909. Dor: 10.1103/ PhysRevC.84.064909. arXiv: 1105.3109 [nucl-ex].

[80] Betty Bezverkhny Abelev et al. In: Phys. Rev. C91 (2015), p. 024609. DoI: 10.1103/PhysRevC.91.024609. arXiv: 1404.0495 [nucl-ex].

[81] Shreyasi Acharya et al. In: Phys. Rev. C99.6 (2019), p. 064901. DOI: 10. 1103/PhysRevC.99.064901. arXiv: 1805.04365 [nucl-ex].

[82] Betty Abelev et al. In: Phys. Rev. C88 (2013), p. 044910. Dor: 10.1103/ PhysRevC.88.044910. arXiv: 1303.0737 [hep-ex].

[83] Ekkard Schnedermann, Josef Sollfrank, and Ulrich W. Heinz. In: Phys. Rev. C48 (1993), pp. 2462-2475. DoI: 10.1103/PhysRevC.48.2462. arXiv: nucl-th/9307020 [nucl-th].

[84] Jaroslav Adam et al. In: Phys. Rev. C93.2 (2016), p. 024917. DOI: 10.1103/ PhysRevC.93.024917. arXiv: 1506.08951 [nucl-ex].

[85] T. Anticic et al. In: Phys. Rev. C94.4 (2016), p. 044906. Dor: 10.1103/ PhysRevC.94.044906. arXiv: 1606.04234 [nucl-ex]. 
[86] Wojciech Broniowski and Wojciech Florkowski. In: Phys. Rev. Lett. 87 (2001), p. 272302. DOI: 10.1103/PhysRevLett.87.272302. arXiv: nuclth/0106050 [nucl-th].

[87] A. G. Knospe, C. Markert, K. Werner, J. Steinheimer, and M. Bleicher. In: Phys. Rev. C93.1 (2016), p. 014911. DoI: 10.1103/PhysRevC.93.014911. arXiv: 1509.07895 [nucl-th].

[88] Sungtae Cho, Taesoo Song, and Su Houng Lee. In: Phys. Rev. C97.2 (2018), p. 024911. DoI: 10.1103/PhysRevC.97.024911. arXiv: 1511.08019 [nucl-th].

[89] J. Steinheimer, J. Aichelin, M. Bleicher, and H. Stoecker. In: Phys. Rev. C95.6 (2017), p. 064902. DOI: 10.1103 / PhysRevC.95.064902. arXiv: 1703.06638 [nucl-th].

[90] Johann Rafelski, Jean Letessier, and Giorgio Torrieri. In: Phys. Rev. C64 (2001). [Erratum: Phys. Rev.C65,069902(2002)], p. 054907. DOI: 10.1103/ PhysRevC.64.054907,10.1103/PhysRevC.65.069902. arXiv: nucl-th/ 0104042 [nucl-th].

[91] Giorgio Torrieri and Johann Rafelski. In: Phys. Lett. B509 (2001), pp. 239245. DOI: 10 . 1016 / S0370 - 2693(01 ) 00492 - 0. arXiv: hep-ph / 0103149 [hep-ph].

[92] C. Markert, G. Torrieri, and Johann Rafelski. In: AIP Conf. Proc. 631.1 (2002), p. 533. DOI: 10.1063/1.1513698. arXiv: hep-ph/0206260 [hep-ph].

[93] M. Tanabashi et al. In: Phys. Rev. D98.3 (2018), p. 030001. DoI: 10.1103/ PhysRevD.98.030001.

[94] H. Bebie, P. Gerber, J. L. Goity, and H. Leutwyler. In: Nucl. Phys. B378 (1992), pp. 95-128. DOI: 10.1016/0550-3213(92)90005-V.

[95] P. Koch, Berndt Muller, and Johann Rafelski. In: Phys. Rept. 142 (1986), pp. 167-262. DOI: 10.1016/0370-1573(86)90096-7.

[96] C. M. Hung and Edward V. Shuryak. In: Phys. Rev. C57 (1998), pp. 18911906. DOI: 10.1103/PhysRevC.57.1891. arXiv: hep-ph/9709264 [hep-ph].

[97] Tetsufumi Hirano and Keiichi Tsuda. In: Phys. Rev. C66 (2002), p. 054905. DOI: 10.1103/PhysRevC.66.054905. arXiv: nucl-th/0205043 [nucl-th].

[98] Peter F. Kolb and Ralf Rapp. In: Phys. Rev. C67 (2003), p. 044903. DoI: 10.1103/PhysRevC.67.044903. arXiv: hep-ph/0210222 [hep-ph]. 
[99] Pasi Huovinen. In: Eur. Phys. J. A37 (2008), pp. 121-128. DOI: 10.1140/ epja/i2007-10611-3. arXiv: 0710.4379 [nucl-th].

[100] Volodymyr Vovchenko, Mark I. Gorenstein, and Horst Stoecker. In: Phys. Rev. C98.3 (2018), p. 034906. DOI: 10.1103/PhysRevC.98.034906. arXiv: 1807.02079 [nucl-th].

[101] https://github.com/vlvovch/Thermal-FIST [Online; accessed 11-July-2020].

[102] Volodymyr Vovchenko, Kai Gallmeister, Jürgen Schaffner-Bielich, and Carsten Greiner. In: Phys. Lett. B 800 (2020), p. 135131. DOI: 10.1016/j. physletb.2019.135131. arXiv: 1903.10024 [hep-ph].

[103] Ralf Rapp. In: Nucl. Phys. A725 (2003), pp. 254-268. Dor: 10.1016/S03759474(03)01581-1. arXiv: hep-ph/0305011 [hep-ph].

[104] Betty Bezverkhny Abelev et al. In: Phys. Rev. Lett. 111 (2013), p. 222301. DOI: 10.1103/PhysRevLett.111.222301. arXiv: 1307.5530 [nucl-ex].

[105] Betty Bezverkhny Abelev et al. In: Phys. Lett. B728 (2014). [Erratum: Phys. Lett.B734,409(2014)], pp. 216-227. DOI: 10.1016/j.physletb.2014.05. 052,10.1016/j.physletb.2013.11.048. arXiv: 1307.5543 [nucl-ex].

[106] Kenneth Aamodt et al. In: Phys. Rev. Lett. 106 (2011), p. 032301. DOI: 10.1103/PhysRevLett.106.032301. arXiv: 1012.1657 [nucl-ex].

[107] Aleksas Mazeliauskas and Vytautas Vislavicius. In: Phys. Rev. C 101.1 (2020), p. 014910. DoI: 10.1103/PhysRevC.101.014910. arXiv: 1907.11059 [hep-ph].

[108] Ivan Melo and Boris Tomášik. In: J. Phys. G 47.4 (2020), p. 045107. DOI: 10.1088/1361-6471/ab5f03. arXiv: 1908.03023 [nucl-th].

[109] Johann Rafelski. In: Phys. Lett. B262 (1991), pp. 333-340. DoI: 10.1016/ 0370-2693(91)91576-H.

[110] F. Becattini, Eduardo Grossi, Marcus Bleicher, Jan Steinheimer, and Reinhard Stock. In: Phys. Rev. C90.5 (2014), p. 054907. DoI: 10.1103/ PhysRevC.90.054907. arXiv: 1405.0710 [nucl-th].

[111] Natasha Sharma, Jean Cleymans, Boris Hippolyte, and Masimba Paradza. In: Phys. Rev. C99.4 (2019), p. 044914. DoI: 10.1103/PhysRevC.99.044914. arXiv: 1811.00399 [hep-ph] . 
[112] Volodymyr Vovchenko, Benjamin Dönigus, and Horst Stoecker. In: Phys. Rev. C 100.5 (2019), p. 054906. DoI: 10.1103/PhysRevC.100.054906. arXiv: 1906.03145 [hep-ph].

[113] Rene Bellwied, Szabolcs Borsanyi, Zoltan Fodor, Sandor D Katz, and Claudia Ratti. In: Phys. Rev. Lett. 111 (2013), p. 202302. Dor: 10.1103/ PhysRevLett.111.202302. arXiv: 1305.6297 [hep-lat].

[114] Fernando Antonio Flor, Gabrielle Olinger, and Rene Bellwied. In: Phys. Lett. $B 814$ (2021), p. 136098. DOI: 10.1016/j.physletb.2021.136098. arXiv: 2009.14781 [nucl-ex].

[115] Granddon D. Yen, Mark I. Gorenstein, Walter Greiner, and Shin-Nan Yang. In: Phys. Rev. C 56 (1997), pp. 2210-2218. Dor: 10.1103/PhysRevC.56.2210. arXiv: nucl-th/9711062.

[116] Volodymyr Vovchenko and Horst Stoecker. In: J. Phys. G44.5 (2017), p. 055103. DOI: 10.1088/1361-6471/aa6409. arXiv: 1512.08046 [hep-ph].

[117] P. Alba, V. Vovchenko, M.I. Gorenstein, and H. Stoecker. In: Nucl. Phys. A 974 (2018), pp. 22-34. DOI: 10.1016/j.nuclphysa.2018.03.007. arXiv: 1606.06542 [hep-ph].

[118] Volodymyr Vovchenko, Attila Pasztor, Zoltan Fodor, Sandor D. Katz, and Horst Stoecker. In: Phys. Lett. B 775 (2017), pp. 71-78. DOI: 10.1016/j. physletb.2017.10.042. arXiv: 1708.02852 [hep-ph].

[119] A. Andronic, P. Braun-Munzinger, J. Stachel, and H. Stoecker. In: Phys. Lett. B697 (2011), pp. 203-207. DOI: 10.1016/j.physletb.2011.01.053. arXiv: 1010.2995 [nucl-th].

[120] Shreyasi Acharya et al. In: Phys. Rev. C99 (2019), p. 024905. DoI: 10.1103/ PhysRevC.99.024905. arXiv: 1805.04361 [nucl-ex].

[121] B. I. Abelev et al. In: Phys. Rev. Lett. 97 (2006), p. 132301. DOI: 10.1103/ PhysRevLett.97.132301. arXiv: nucl-ex/0604019 [nucl-ex].

[122] S. Jeon and V. Koch. In: Phys. Rev. Lett. 83 (1999), pp. 5435-5438. DoI: 10.1103/PhysRevLett.83.5435. arXiv: nucl-th/9906074 [nucl-th].

[123] Giorgio Torrieri, Rene Bellwied, Christina Markert, and Gary Westfall. In: J. Phys. G37 (2010), p. 094016. DOI: 10.1088/0954-3899/37/9/094016. arXiv: 1001.0087 [nucl-th]. 
[124] R. Hagedorn and Johann Rafelski. In: Phys. Lett. B 97 (1980), p. 136. DoI: 10.1016/0370-2693(80)90566-3.

[125] Mark I. Gorenstein, V.K. Petrov, and G.M. Zinovev. In: Phys. Lett. B 106 (1981), pp. 327-330. DOI: 10.1016/0370-2693(81)90546-3.

[126] R. Hagedorn. In: Z. Phys. C 17 (1983), p. 265. DOI: 10.1007/BF01578153.

[127] Joseph I. Kapusta and Keith A. Olive. In: Nucl. Phys. A 408 (1983), pp. 478-494. DOI: 10.1016/0375-9474(83)90241-5.

[128] Dirk H. Rischke, Mark I. Gorenstein, Horst Stoecker, and Walter Greiner. In: Z. Phys. C 51 (1991), pp. 485-490. DOI: 10.1007/BF01548574.

[129] D. Anchishkin. In: Sov. Phys. JETP 75 (1992), pp. 195-199.

[130] Dmitry Anchishkin and Esko Suhonen. In: Nucl. Phys. A 586 (1995), pp. 734-754. DOI: 10.1016/0375-9474(94)00822-5.

[131] J. E. Mayer and M. G. Mayer. Statistical mechanics. New York: John Wiley \& Sons, 1977.

[132] K. Huang. Statistical Mechanics. New York: John Wiley \& Sons, 1963.

[133] W. Greiner, L. Neise, and H. Stöcker. Thermodynamics and Statistical Mechanics. Inc: Springer-Verlag New York, 1995.

[134] Roger Dashen, Shang-Keng Ma, and Herbert J. Bernstein. In: Phys. Rev. 187 (1969), pp. 345-370. DOI: 10.1103/PhysRev.187.345.

[135] R. Venugopalan and M. Prakash. In: Nucl. Phys. A 546 (1992), pp. 718-760. DOI: 10.1016/0375-9474(92)90005-5.

[136] A. Kostyuk, M. Gorenstein, Horst Stoecker, and W. Greiner. In: Phys. Rev. C 63 (2001), p. 044901. DOI: 10.1103/PhysRevC.63.044901. arXiv: hep-ph/0004163.

[137] L. I. Schiff. Quantum Mechanics. McGraw-Hill, 1968.

[138] E. Beth and G. Uhlenbeck. In: Physica 4 (1937), pp. 915-924. DOI: 10. 1016/S0031-8914(37)80189-5.

[139] Granddon D. Yen and Mark I. Gorenstein. In: Phys. Rev. C 59 (1999), pp. 2788-2791. DOI: 10.1103/PhysRevC.59.2788. arXiv: nucl-th/9808012.

[140] P. Braun-Munzinger, I. Heppe, and J. Stachel. In: Phys. Lett. B 465 (1999), pp. 15-20. DOI: 10.1016/S0370-2693(99)01076-X. arXiv: nucl-th/9903010. 
[141] A. Andronic, P. Braun-Munzinger, J. Stachel, and M. Winn. In: Phys. Lett. B 718 (2012), pp. 80-85. DOI: 10.1016/j.physletb.2012.10.001. arXiv: 1201.0693 [nucl-th].

[142] V.V. Begun, M. Gazdzicki, and M.I. Gorenstein. In: Phys. Rev. C 88.2 (2013), p. 024902. DoI: 10.1103/PhysRevC.88.024902. arXiv: 1208.4107 [nucl-th].

[143] Abhijit Bhattacharyya, Supriya Das, Sanjay K. Ghosh, Rajarshi Ray, and Subhasis Samanta. In: Phys. Rev. C 90.3 (2014), p. 034909. DoI: 10.1103/PhysRevC.90.034909. arXiv: 1310.2793 [hep-ph].

[144] V. Vovchenko, D.V. Anchishkin, and M.I. Gorenstein. In: Phys. Rev. C 91.2 (2015), p. 024905. DOI: 10.1103/PhysRevC.91.024905. arXiv: 1412.5478 [nucl-th].

[145] Krzysztof Redlich and Kacper Zalewski. In: Phys. Rev. C 93.1 (2016), p. 014910. DOI: 10.1103/PhysRevC.93.014910. arXiv: 1507.05433 [hep-ph].

[146] D. Anchishkin and V. Vovchenko. In: J. Phys. G 42.10 (2015), p. 105102. DOI: 10.1088/0954-3899/42/10/105102. arXiv: 1411.1444 [nucl-th].

[147] Guru Prakash Kadam and Hiranmaya Mishra. In: Phys. Rev. C 92.3 (2015), p. 035203. DOI: 10.1103/PhysRevC.92.035203. arXiv: 1506.04613 [hep-ph].

[148] S. Typel. In: Eur. Phys. J. A 52.1 (2016), p. 16. DoI: 10.1140/epja/i201616016-3.

[149] Pok Man Lo, Bengt Friman, Michal Marczenko, Krzysztof Redlich, and Chihiro Sasaki. In: Phys. Rev. C 96.1 (2017), p. 015207. Dor: 10.1103/ PhysRevC.96.015207. arXiv: 1703.00306 [nucl-th].

[150] Madappa Prakash, Manju Prakash, R. Venugopalan, and G. Welke. In: Phys. Rept. 227 (1993), pp. 321-366. DOI: 10.1016/0370-1573(93)90092-R.

[151] Robert B. Wiringa, V.G.J. Stoks, and R. Schiavilla. In: Phys. Rev. C 51 (1995), pp. 38-51. DOI: 10.1103/PhysRevC.51.38. arXiv: nucl-th/9408016.

[152] Pasi Huovinen and Peter Petreczky. In: Phys. Lett. B 777 (2018), pp. 125130. DOI: 10.1016/j.physletb.2017.12.001. arXiv: 1708.00879 [hep-ph].

[153] V. Vovchenko, D.V. Anchishkin, and M.I. Gorenstein. In: Phys. Rev. C 91.6 (2015), p. 064314. DOI: 10.1103/PhysRevC.91.064314. arXiv: 1504.01363 [nucl-th]. 
[154] C.J. Horowitz and A. Schwenk. In: Nucl. Phys. A 776 (2006), pp. 55-79. DOI: 10.1016/j.nuclphysa.2006.05.009. arXiv: nucl-th/0507033.

[155] Volodymyr Vovchenko, Mark I. Gorenstein, and Horst Stoecker. In: Phys. Rev. Lett. 118.18 (2017), p. 182301. DOI: 10.1103/PhysRevLett.118.182301. arXiv: 1609.03975 [hep-ph].

[156] Takashi Inoue, Sinya Aoki, Takumi Doi, Tetsuo Hatsuda, Yoichi Ikeda, Noriyoshi Ishii, Keiko Murano, Hidekatsu Nemura, and Kanji Sasaki. In: Nucl. Phys. A 881 (2012). Ed. by Avraham Gal, Osamu Hashimoto, and Josef Pochodzalla, pp. 28-43. DOI: 10.1016/j.nuclphysa.2012.02.008. arXiv: 1112.5926 [hep-lat].

[157] Shigenori Kagiyama, Akihiro Nakamura, and Toshihiro Omodaka. In: Z. Phys. C 53 (1992), pp. 163-168. DOI: 10.1007/BF01483885.

[158] L. Ferroni and V. Koch. In: Phys. Rev. C 79 (2009), p. 034905. DoI: 10.1103/PhysRevC.79.034905. arXiv: 0812.1044 [nucl-th].

[159] C. Patrignani et al. In: Chin. Phys. C 40.10 (2016), p. 100001. DoI: 10. 1088/1674-1137/40/10/100001.

[160] Francesco Becattini. In: Z. Phys. C 69.3 (1996), pp. 485-492. DOI: 10.1007/ BF02907431.

[161] S. Wheaton and J. Cleymans. In: Comput. Phys. Commun. 180 (2009), pp. 84-106. DOI: 10.1016/j.cpc.2008.08.001. arXiv: hep-ph/0407174.

[162] Szabolcs Borsanyi, Zoltan Fodor, Sandor D. Katz, Stefan Krieg, Claudia Ratti, and Kalman Szabo. In: JHEP 01 (2012), p. 138. DOI: 10.1007/ JHEP01(2012)138. arXiv: 1112.4416 [hep-lat] .

[163] R. Bellwied, S. Borsanyi, Z. Fodor, S. D. Katz, A. Pasztor, C. Ratti, and K. K. Szabo. In: Phys. Rev. D92.11 (2015), p. 114505. DoI: 10.1103 / PhysRevD.92.114505. arXiv: 1507.04627 [hep-lat].

[164] A. Bazavov et al. In: Phys. Rev. D95.5 (2017), p. 054504. DoI: 10.1103/ PhysRevD.95.054504. arXiv: 1701.04325 [hep-lat].

[165] A. Bazavov et al. In: Phys. Rev. D96.7 (2017), p. 074510. DoI: 10.1103/ PhysRevD.96.074510. arXiv: 1708.04897 [hep-lat].

[166] Volodymyr Vovchenko. In: Phys. Rev. C 96.1 (2017), p. 015206. DOI: 10.1103/PhysRevC.96.015206. arXiv: 1701.06524 [nucl-th]. 
[167] P. Papazoglou, D. Zschiesche, S. Schramm, J. Schaffner-Bielich, Horst Stoecker, and W. Greiner. In: Phys. Rev. C59 (1999), pp. 411-427. DoI: 10.1103/PhysRevC.59.411. arXiv: nucl-th/9806087 [nucl-th].

[168] J. Steinheimer, S. Schramm, and H. Stoecker. In: J. Phys. G38 (2011), p. 035001. DOI: $10.1088 / 0954-3899 / 38 / 3 / 035001$. arXiv: 1009.5239 [hep-ph].

[169] Carleton E. Detar and Teiji Kunihiro. In: Phys. Rev. D39 (1989), p. 2805. DOI: 10.1103/PhysRevD.39.2805.

[170] Kenji Fukushima. In: Phys. Lett. B591 (2004), pp. 277-284. DOI: 10.1016/j. physletb.2004.04.027. arXiv: hep-ph/0310121 [hep-ph].

[171] J. Steinheimer, S. Schramm, and H. Stoecker. In: Phys. Rev. C84 (2011), p. 045208. DOI: 10.1103/PhysRevC.84.045208. arXiv: 1108.2596 [hep-ph].

[172] D. Zschiesche, L. Tolos, Jurgen Schaffner-Bielich, and Robert D. Pisarski. In: Phys. Rev. C 75 (2007), p. 055202. Dor: 10.1103/PhysRevC.75.055202. arXiv: nucl-th/0608044.

[173] Chihiro Sasaki. In: Nucl. Phys. A 970 (2018), pp. 388-397. DoI: 10.1016/j. nuclphysa.2018.01.004. arXiv: 1707.05081 [hep-ph].

[174] V.A. Dexheimer and S. Schramm. In: Phys. Rev. C 81 (2010), p. 045201. DOI: 10.1103/PhysRevC.81.045201. arXiv: 0901.1748 [astro-ph.SR].

[175] Yuichi Motohiro, Youngman Kim, and Masayasu Harada. In: Phys. Rev. C 92.2 (2015). [Erratum: Phys.Rev.C 95, 059903 (2017)], p. 025201. DOI: 10.1103/PhysRevC.92.025201. arXiv: 1505.00988 [nucl-th].

[176] Gordon Baym, Christopher Pethick, and Peter Sutherland. In: Astrophys. J. 170 (1971), pp. 299-317. DOI: 10.1086/151216.

[177] Szabocls Borsanyi, Zoltan Fodor, Christian Hoelbling, Sandor D. Katz, Stefan Krieg, and Kalman K. Szabo. In: Phys. Lett. B730 (2014), pp. 99104. DOI: 10.1016/j.physletb.2014.01.007. arXiv: 1309.5258 [hep-lat].

[178] Kenji Fukushima. In: Phys. Rev. D77 (2008). [Erratum: Phys. Rev.D78,039902(2008)], p. 114028. DOI: 10.1103/PhysRevD.77.114028,10.1103/PhysRevD.78. 039902. arXiv: 0803.3318 [hep-ph].

[179] M. Albright, J. Kapusta, and C. Young. In: Phys. Rev. C90.2 (2014), p. 024915. DOI: 10.1103/PhysRevC.90.024915. arXiv: 1404.7540 [nucl-th]. 
[180] A. Mukherjee, J. Steinheimer, and S. Schramm. In: Phys. Rev. C96.2 (2017), p. 025205. DOI: 10.1103/PhysRevC.96.025205. arXiv: 1611.10144 [nucl-th].

[181] Claudia Ratti, Michael A. Thaler, and Wolfram Weise. In: Phys. Rev. D73 (2006), p. 014019. DOI: 10.1103/PhysRevD.73.014019. arXiv: hepph/0506234 [hep-ph].

[182] M. A. Stephanov. In: Phys. Rev. Lett. 102 (2009), p. 032301. DoI: 10.1103/ PhysRevLett.102.032301. arXiv: 0809.3450 [hep-ph].

[183] D. Zschiesche, G. Zeeb, S. Schramm, and Horst Stoecker. In: J. Phys. G31 (2005), pp. 935-946. DOI: 10.1088/0954-3899/31/8/022. arXiv: nucl-th/0407117 [nucl-th].

[184] Stefan Floerchinger and Mauricio Martinez. In: Phys. Rev. C92.6 (2015), p. 064906. DOI: 10.1103/PhysRevC.92.064906. arXiv: 1507.05569 [nucl-th].

[185] J. Steinheimer and S. Schramm. In: Phys. Lett. B696 (2011), pp. 257-261. DOI: 10.1016/j.physletb.2010.12.046. arXiv: 1005.1176 [hep-ph] .

[186] Jan Steinheimer and Stefan Schramm. In: Phys. Lett. B736 (2014), pp. 241245. DOI: 10.1016/j.physletb.2014.07.018. arXiv: 1401.4051 [nucl-th].

[187] C.R. Allton, S. Ejiri, S.J. Hands, O. Kaczmarek, F. Karsch, E. Laermann, C. Schmidt, and L. Scorzato. In: Phys. Rev. D 66 (2002), p. 074507. Dor: 10.1103/PhysRevD.66.074507. arXiv: hep-lat/0204010.

[188] Volodymyr Vovchenko, Jan Steinheimer, Owe Philipsen, and Horst Stoecker. In: Phys. Rev. D 97.11 (2018), p. 114030. DoI: 10.1103/PhysRevD.97. 114030. arXiv: 1711.01261 [hep-ph].

[189] Andre Roberge and Nathan Weiss. In: Nucl. Phys. B 275 (1986), pp. 734745. DOI: 10.1016/0550-3213(86)90582-1.

[190] Maria Paola Lombardo. In: PoS LAT2005 (2006). Ed. by Christopher Michael, p. 168. DoI: 10.22323/1.020.0168. arXiv: hep-lat/0509181.

[191] Horst Stoecker, W. Greiner, and W. Scheid. In: Z. Phys. A286 (1978), p. 121. DOI: 10.1007/BF01434620.

[192] A. H. Taub. In: Phys. Rev. 74 (1948), pp. 328-334. DoI: 10.1103/PhysRev. 74.328 .

[193] K. S. Thorne. In: 179 (Feb. 1973), pp. 897-908. DOI: 10.1086/151927. 
[194] Horst Stoecker and W. Greiner. In: Phys. Rept. 137 (1986), pp. 277-392. DOI: 10.1016/0370-1573(86)90131-6.

[195] Laszlo P. Csernai, Joseph.I. Kapusta, and Larry D. McLerran. In: Phys. Rev. Lett. 97 (2006), p. 152303. DOI: 10.1103/PhysRevLett.97.152303. arXiv: nucl-th/0604032 [nucl-th].

[196] Paul Romatschke and Ulrike Romatschke. In: Phys. Rev. Lett. 99 (2007), p. 172301. DOI: 10.1103/PhysRevLett.99.172301. arXiv: 0706.1522 [nucl-th].

[197] J. Steinheimer, M. Bleicher, H. Petersen, S. Schramm, H. Stoecker, and D. Zschiesche. In: Phys. Rev. C77 (2008), p. 034901. DoI: 10.1103/PhysRevC. 77.034901. arXiv: 0710.0332 [nucl-th].

[198] H. G. Baumgardt, J. U. Schott, Y. Sakamoto, E. Schopper, Horst Stoecker, J. Hofmann, W. Scheid, and W. Greiner. In: Z. Phys. A273 (1975), pp. 359371. DOI: $10.1007 / \mathrm{BF} 01435578$.

[199] Horst Stoecker, G. Graebner, J. A. Maruhn, and W. Greiner. In: Phys. Lett. 95B (1980), pp. 192-197. DOI: 10.1016/0370-2693(80)90467-0.

[200] Horst Stoecker, A. A. Ogloblin, and W. Greiner. In: Z. Phys. A303 (1981), pp. 259-266. DOI: 10.1007/BF01421522.

[201] Horst Stoecker, M. Gyulassy, and J. Boguta. In: Phys. Lett. 103B (1981), pp. 269-274. DOI: 10.1016/0370-2693(81)90222-7.

[202] D. Hahn and Horst Stoecker. In: Nucl. Phys. A476 (1988), pp. 718-772. DOI: 10.1016/0375-9474(88)90332-6.

[203] A. V. Merdeev, L. M. Satarov, and I. N. Mishustin. In: Phys. Rev. C84 (2011), p. 014907. DOI: 10.1103/PhysRevC.84.014907. arXiv: 1103.3988 [hep-ph].

[204] Matthias Hanauske, Jan Steinheimer, Luke Bovard, Ayon Mukherjee, Stefan Schramm, Kentaro Takami, Jens Papenfort, Natascha Wechselberger, Luciano Rezzolla, and Horst Stoecker. In: J. Phys. Conf. Ser. 878.1 (2017), p. 012031. DOI: 10.1088/1742-6596/878/1/012031.

[205] Elias R. Most, L. Jens Papenfort, Veronica Dexheimer, Matthias Hanauske, Stefan Schramm, Horst Stoecker, and Luciano Rezzolla. In: Phys. Rev. Lett. 122.6 (2019), p. 061101. DOI: 10.1103/PhysRevLett.122.061101. arXiv: 1807.03684 [astro-ph.HE]. 
[206] Kenji Fukushima. In: Phys. Rev. C91.4 (2015), p. 044910. DOI: 10.1103/ PhysRevC.91.044910. arXiv: 1409.0698 [hep-ph].

[207] Volodymyr Vovchenko, Lijia Jiang, Mark I. Gorenstein, and Horst Stoecker. In: Phys. Rev. C98.2 (2018), p. 024910. DOI: 10.1103/PhysRevC.98.024910. arXiv: 1711.07260 [nucl-th].

[208] Tim Dietrich, Sebastiano Bernuzzi, Maximiliano Ujevic, and Bernd Brugmann. In: Phys. Rev. D91.12 (2015), p. 124041. DoI: 10.1103/PhysRevD. 91.124041. arXiv: 1504.01266 [gr-qc].

[209] David Radice, Sebastiano Bernuzzi, Walter Del Pozzo, Luke F. Roberts, and Christian D. Ott. In: Astrophys. J. 842.2 (2017), p. L10. DOI: 10.3847/20418213/aa775f. arXiv: 1612.06429 [astro-ph.HE].

[210] Andreas Bauswein, Niels-Uwe F. Bastian, David B. Blaschke, Katerina Chatziioannou, James A. Clark, Tobias Fischer, and Micaela Oertel. In: Phys. Rev. Lett. 122.6 (2019), p. 061102. Dor: 10.1103/PhysRevLett.122. 061102. arXiv: 1809.01116 [astro-ph.HE].

[211] C. Greiner, P. Koch, and Horst Stoecker. In: Phys. Rev. Lett. 58 (1987), pp. 1825-1828. DOI: 10.1103/PhysRevLett.58.1825.

[212] Matthias Hempel, Veronica Dexheimer, Stefan Schramm, and Igor Iosilevskiy. In: Phys. Rev. C88.1 (2013), p. 014906. DoI: 10.1103/PhysRevC.88.014906. arXiv: 1302.2835 [nucl-th].

[213] Roman Poberezhnyuk, Volodymyr Vovchenko, Mark I. Gorenstein, and Horst Stoecker. In: Phys. Rev. C99.2 (2019), p. 024907. Dor: 10.1103/ PhysRevC.99.024907. arXiv: 1810.07640 [hep-ph].

[214] Richard C. Tolman. In: Phys. Rev. 55 (1939), pp. 364-373. DoI: 10.1103/ PhysRev.55.364.

[215] J. R. Oppenheimer and G. M. Volkoff. In: Phys. Rev. 55 (1939), pp. 374381. DOI: 10.1103/PhysRev.55.374.

[216] Sanjin Benic, David Blaschke, David E. Alvarez-Castillo, Tobias Fischer, and Stefan Typel. In: Astron. Astrophys. 577 (2015), A40. DOI: 10.1051/ 0004-6361/201425318. arXiv: 1411.2856 [astro-ph.HE] .

[217] Mark Alexander Randolph Kaltenborn, Niels-Uwe Friedrich Bastian, and David Bernhard Blaschke. In: Phys. Rev. D96.5 (2017), p. 056024. DOI: 10.1103/PhysRevD.96.056024. arXiv: 1701.04400 [astro-ph.HE]. 
[218] Tobias Fischer, Niels-Uwe F. Bastian, Meng-Ru Wu, Petr Baklanov, Elena Sorokina, Sergei Blinnikov, Stefan Typel, Thomas Klähn, and David B. Blaschke. In: Nat. Astron. 2.12 (2018), pp. 980-986. DOI: 10.1038/s41550018-0583-0. arXiv: 1712.08788 [astro-ph.HE].

[219] Yifan Song, Gordon Baym, Tetsuo Hatsuda, and Toru Kojo. In: (2019). arXiv: 1905.01005 [astro-ph.HE].

[220] Larry McLerran and Sanjay Reddy. In: Phys. Rev. Lett. 122.12 (2019), p. 122701. DOI: 10.1103/PhysRevLett.122.122701. arXiv: 1811.12503 [nucl-th].

[221] Sanjin Benic, Igor Mishustin, and Chihiro Sasaki. In: Phys. Rev. D91.12 (2015), p. 125034. DOI: 10.1103/PhysRevD.91.125034. arXiv: 1502.05969 [hep-ph].

[222] Michał Marczenko, David Blaschke, Krzysztof Redlich, and Chihiro Sasaki. In: Phys. Rev. D98.10 (2018), p. 103021. DOI: 10.1103/PhysRevD.98.103021. arXiv: 1805.06886 [nucl-th].

[223] Tanja Hinderer, Benjamin D. Lackey, Ryan N. Lang, and Jocelyn S. Read. In: Phys. Rev. D81 (2010), p. 123016. DOI: 10.1103/PhysRevD.81.123016. arXiv: 0911.3535 [astro-ph.HE].

[224] B. P. Abbott et al. In: Phys. Rev. Lett. 119.16 (2017), p. 161101. DoI: 10.1103/PhysRevLett.119.161101. arXiv: 1710.05832 [gr-qc].

[225] Eemeli Annala, Tyler Gorda, Aleksi Kurkela, and Aleksi Vuorinen. In: Phys. Rev. Lett. 120.17 (2018), p. 172703. DOI: 10.1103/PhysRevLett.120.172703. arXiv: 1711.02644 [astro-ph.HE].

[226] Elias R. Most, Lukas R. Weih, Luciano Rezzolla, and Jürgen SchaffnerBielich. In: Phys. Rev. Lett. 120.26 (2018), p. 261103. DOI: 10.1103 / PhysRevLett.120.261103. arXiv: 1803.00549 [gr-qc].

[227] B. P. Abbott et al. In: Phys. Rev. X9.1 (2019), p. 011001. DoI: 10.1103/ PhysRevX.9.011001. arXiv: 1805.11579 [gr-qc].

[228] B. P. Abbott et al. In: Phys. Rev. Lett. 121.16 (2018), p. 161101. DoI: 10.1103/PhysRevLett.121.161101. arXiv: 1805.11581 [gr-qc].

[229] Volker Koch. "Hadronic Fluctuations and Correlations". In: Relativistic Heavy Ion Physics. Ed. by R. Stock. 2010, pp. 626-652. DOI: 10.1007/9783-642-01539-7\_20. arXiv: 0810.2520 [nucl-th]. 
[230] A. Bazavov et al. In: Phys. Rev. D 86 (2012), p. 034509. DoI: 10.1103/ PhysRevD.86.034509. arXiv: 1203.0784 [hep-lat].

[231] Volodymyr Vovchenko, Anton Motornenko, Paolo Alba, Mark I. Gorenstein, Leonid M. Satarov, and Horst Stoecker. In: Phys. Rev. C 96.4 (2017), p. 045202. DOI: 10.1103/PhysRevC.96.045202. arXiv: 1707.09215 [nucl-th].

[232] J. Schaffner, Horst Stoecker, and C. Greiner. In: Phys. Rev. C 46 (1992), pp. 322-329. DOI: 10.1103/PhysRevC.46.322.

[233] Jurgen Schaffner, Carl B. Dover, Avraham Gal, Carsten Greiner, and Horst Stoecker. In: Phys. Rev. Lett. 71 (1993), pp. 1328-1331. DOI: 10.1103/ PhysRevLett.71.1328.

[234] E.P. Gilson and R.L. Jaffe. In: Phys. Rev. Lett. 71 (1993), pp. 332-335. DOI: 10.1103/PhysRevLett.71.332. arXiv: hep-ph/9302270.

[235] Jurgen Schaffner-Bielich, Carsten Greiner, Alexander Diener, and Horst Stoecker. In: Phys. Rev. C 55 (1997), pp. 3038-3046. DOI: 10.1103 / PhysRevC.55.3038. arXiv: nucl-th/9611052.

[236] Stefan Scherer, Marcus Bleicher, Stephane Haussler, and Horst Stocker. In: Int. J. Mod. Phys. E 17 (2008), pp. 965-1014. DOI: 10.1142/S0218301308010386.

[237] Jan Steinheimer, Michael Mitrovski, Tim Schuster, Hannah Petersen, Marcus Bleicher, and Horst Stoecker. In: Phys. Lett. B 676 (2009), pp. 126-131. DOI: 10.1016/j.physletb.2009.04.062. arXiv: 0811.4077 [hep-ph].

[238] A.S. Botvina, J. Steinheimer, E. Bratkovskaya, M. Bleicher, and J. Pochodzalla. In: Phys. Lett. B 742 (2015), pp. 7-14. DOI: 10.1016/j.physletb.2014. 12.060. arXiv: 1412.6665 [nucl-th].

[239] Florian Seck, Tetyana Galatyuk, Ayon Mukherjee, Ralf Rapp, Jan Steinheimer, and Joachim Stroth. In: (Oct. 2020). arXiv: 2010.04614 [nucl-th].

[240] Pawel Danielewicz, Roy Lacey, and William G. Lynch. In: Science 298 (2002), pp. 1592-1596. DOI: 10.1126/science.1078070. arXiv: nucl-th/ 0208016 .

[241] Yasushi Nara and Horst Stoecker. In: Phys. Rev. C 100.5 (2019), p. 054902. DOI: 10.1103/PhysRevC.100.054902. arXiv: 1906.03537 [nucl-th]. 
[242] Yasushi Nara, Tomoyuki Maruyama, and Horst Stoecker. In: Phys. Rev. C 102.2 (2020), p. 024913. DOI: 10.1103/PhysRevC.102.024913. arXiv: 2004.05550 [nucl-th].

[243] Jan Steinheimer, Jörg Aichelin, and Marcus Bleicher. In: Phys. Rev. Lett. 110.4 (2013), p. 042501. DOI: 10.1103/PhysRevLett.110.042501. arXiv: 1203.5302 [nucl-th].

[244] Rene Bellwied, Jacquelyn Noronha-Hostler, Paolo Parotto, Israel Portillo Vazquez, Claudia Ratti, and Jamie M. Stafford. In: Phys. Rev. C 99.3 (2019), p. 034912. DoI: 10.1103/PhysRevC.99.034912. arXiv: 1805.00088 [hep-ph].

[245] Lukas R. Weih, Matthias Hanauske, and Luciano Rezzolla. In: Phys. Rev. Lett. 124.17 (2020), p. 171103. DOI: 10.1103/PhysRevLett.124.171103. arXiv: 1912.09340 [gr-qc].

[246] Mark G. Alford and Sophia Han. In: Eur. Phys. J. A 52.3 (2016), p. 62. DOI: 10.1140/epja/i2016-16062-9. arXiv: 1508.01261 [nucl-th].

[247] Andreas Zacchi, Laura Tolos, and Jürgen Schaffner-Bielich. In: Phys. Rev. D 95.10 (2017), p. 103008. DOI: 10.1103/PhysRevD.95.103008. arXiv: 1612.06167 [astro-ph.HE].

[248] Gloria Montana, Laura Tolos, Matthias Hanauske, and Luciano Rezzolla. In: Phys. Rev. D 99.10 (2019), p. 103009. DOI: 10.1103/PhysRevD.99.103009. arXiv: 1811.10929 [astro-ph.HE].

[249] Eemeli Annala, Tyler Gorda, Aleksi Kurkela, Joonas Nättilä, and Aleksi Vuorinen. In: Nature Phys. (2020). DOI: 10.1038/s41567-020-0914-9. arXiv: 1903.09121 [astro-ph.HE].

[250] P. Jakobus, A. Motornenko, R.O. Gomes, J. Steinheimer, and H. Stoecker. In: (Apr. 2020). arXiv: 2004.07026 [nucl-th].

[251] Hung Tan, Jacquelyn Noronha-Hostler, and Nico Yunes. In: (June 2020). arXiv: 2006.16296 [astro-ph.HE].

[252] Thomas E. Riley et al. In: Astrophys. J. Lett. 887.1 (2019), p. L21. DOI: 10.3847/2041-8213/ab481c. arXiv: 1912.05702 [astro-ph.HE] .

[253] M.C. Miller et al. In: Astrophys. J. Lett. 887.1 (2019), p. L24. DOI: 10. 3847/2041-8213/ab50c5. arXiv: 1912.05705 [astro-ph.HE] . 
[254] M. Punturo et al. In: Class. Quant. Grav. 27 (2010). Ed. by Zsuzsa Marka and Szabolcs Marka, p. 084007. DOI: 10.1088/0264-9381/27/8/084007.

[255] Benjamin P Abbott et al. In: Class. Quant. Grav. 34.4 (2017), p. 044001. DOI: 10.1088/1361-6382/aa51f4. arXiv: 1607.08697 [astro-ph.IM] .

[256] Isaac Vidaña. In: Proc. Roy. Soc. Lond. A A474 (2018), p. 0145. DOI: 10.1098/rspa.2018.0145. arXiv: 1803.00504 [nucl-th].

[257] V. A. Ambartsumyan and G. S. Saakyan. In: Astronomicheskii Zhurnal 37 (Oct. 1960), p. 193.

[258] Eduardo S. Fraga, Aleksi Kurkela, and Aleksi Vuorinen. In: Astrophys. J. Lett. 781.2 (2014), p. L25. DOI: 10.1088/2041-8205/781/2/L25. arXiv: 1311.5154 [nucl-th].

[259] Aleksi Kurkela, Paul Romatschke, and Aleksi Vuorinen. In: Phys. Rev. D81 (2010), p. 105021. DOI: 10.1103/PhysRevD.81.105021. arXiv: 0912.1856 [hep-ph].

[260] https://github.com/amotornenko/TOVsolver [Online; accessed 21-Sep2020].

[261] V. Dexheimer, J. Steinheimer, R. Negreiros, and S. Schramm. In: Phys. Rev. C87.1 (2013), p. 015804. DOI: 10.1103/PhysRevC.87.015804. arXiv: 1206.3086 [astro-ph.HE]. 


\section{Acknowledgments}

I would like to express my gratitude to everyone who directly and indirectly helped and supported me through the years of my doctoral studies in Frankfurt.

First of all, I thank Prof. Dr. Horst Stöcker for inviting me to Frankfurt and for getting me involved in numerous scientific activities at FIAS. It is incredible how much synergy is produced by your encouragement to cooperate. Your guidance and support can never be overestimated. Special thanks for the middle-of-thenight emails full of exciting scientific ideas, many of which were implemented in this thesis.

I would like to thank the late Prof. Dr. Stefan Schramm for involving me in the CMF activities. I wish we could work longer together. I am grateful that I had an opportunity to work with him. I learned a lot during this short time.

This thesis wouldn't have even been started without Prof. Dr. Mark Gorenstein, my supervisor during my Bachelor's and Master's studies in Kyiv. I'm thankful that I was able to share Mark's infinite passion and curiosity for physics. Huge thanks for bringing me to the physics of QCD matter!

Thanks to Prof. Dr. Marcus Bleicher, for agreeing to read and review this thesis.

To all my colleagues from FIAS and other institutions who shapened my scientific carrer path.

I thank Dr. Jan Steinheimer, a friend and a senior experienced colleague. For any question I have, either about physics or living in Germany you are always there to help. Special thanks for the BBQs and bringing people together (in the good old times when it was allowed).

To a friend, office-mate, travel-mate and a talented scientist Dr. Volodya Vovchenko. Thank you for productive and sometimes just fun conversations and for all the projects done together.

The person who supported me on an everyday basis through the whole period of the studies is my wife, my love, Mariia. It is hard to express how grateful I am to you. For your support and understanding, for your advice and comments, for caring that I'm not overworking or overeating, for always being with me... I'm so happy to have you!

The last but the biggest thanks to my parents, for their constant support during my physics studies and my whole life. No matter if I'm a few meters or thousands of kilometers away I feel your support. Thank you for everything! 


\section{Anton MOTORNENKO}

\section{Persönliche Daten}

GEBURTSORT UND -DATUM:

FAMILIENSTAND:

NATIONALITÄT:

ANSCHRIFT:

TELEFON:

EMAIL:

PROFIL IN DER INSPIRE-DATENBANK:

Kiew, Ukraine | 21 Februar 1994

Verheiratet

Ukrainisch

32 Kurmainzer, 61440 Oberursel, DE

+4916093327532

hello@motornenko.com

motornenko@fias.uni-frankfurt.de

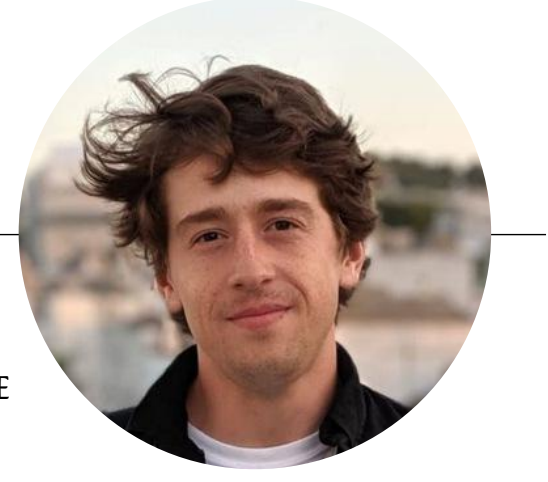

SPRACHEN

http://inspirehep.net/author/profile/A.Motornenko.1

Ukrainisch (Muttersprache), Russisch (Muttersprache),

Englisch (fließend), Deutsch (Grundkenntnisse)

\section{WISSENSCHAFTLICHE INTERESSEN}

- Gravitationswellen

- Kernphysik

- Maschinelles Lernen und künstliche Intelligenz

- Molekulardynamik

- Monte-Carlo-Simulationen
- Neutronensterne

- QCD-Phänomenologie und Phasendiagramm

- Relativistische Fluiddynamik

- Schwerionenkollisionen

- Wissenschaftliches Rechnen und Hochleistungsrechnen

\section{AUSBILDUNG}

2017-2021 Promotionsstudium, Fachbereich PHYSIK

Johann Wolfgang Goethe-Universität Frankfurt am Main, Deutschland

2017-2021 Stipendiat

Frankfurt Institute for Advanced Studies, Frankfurt am Main, Deutschland

2015-2017 MSc in PhysIK, Fachgebiet: QUANTENFELDTHEorIE

Nationale Taras-Schewtschenko-Universität Kiew, Ukraine

MASTERARBEIT:"Statistische und dynamische Eigenschaften der hadronischen Materie"

Betreuer: Prof. Mark GoRensteIN und Prof. Larissa BRAVINA

2017-2017 Austauschstudent (Wintersemester)

Universität Oslo, Norwegen

2011-2015 BSC in PHYSIK, Fachgebiet: QUANTENFELDTHEORIE

Nationale Taras-Schewtschenko-Universität Kiew, Ukraine

\section{ARBEITSERFAHRUNG}

2018-2020 Tutor "C ++, Scientific Computing, and Machine Learning"

Johann Wolfgang Goethe-Universität Frankfurt am Main, Deutschland

2016

Sommerpraktikant

Deutsches Elektronen-Synchrotron, Hamburg, Deutschland

\section{AUSZEICHNUNGEN}

Окт. 2020 Giersch excellence award für herausragende Leistungen während der Promotion

Окт. 2019 Giersch excellence award für herausragende Leistungen während der Promotion

JUNI 2018 Richard Feynman diploma und

Auszeichnung für den besten theoretischen Vortrag

INTERNATIONAL SCHOOl of SUbNUClear Physics 56 KURS in Ettore Majorana Foundation and Center

DEZ. 2016 Auszeichnung für den besten theoretischen Vortrag

Young Scientists Conference "Probleme von Theoretische Physik" am BogolyubovInstitut für Theoretische Physik

\section{COMPUTERKENNTNISSE}

Bash shell scripting, $\mathrm{C} / \mathrm{C}++_{+}$, cluster computing, Git, HTML, Java, JavaScript,

Linux, $\mathrm{LAT}_{\mathrm{E}} \mathrm{X}$, modern Fortran, Maschinelles Lernen, OpenGL,

parallele Berechnungen, Python, Tensorflow, Wolfram Mathematica 


\begin{tabular}{lllc}
$\begin{array}{l}\text { Zusammenfassung*: } \\
\text { PAPIERE }\end{array}$ & ZITATE & H-INDEX & ZITATE/PAPIER \\
\hline 26 & 223 & 9 & $\mathbf{8 . 6}$ \\
& & & $*$ gemäß iNSPIRE-Datenbank vom März 2021
\end{tabular}

Liste:

26 A. Motornenko, S. Pal, A. Bhattacharyya, J. Steinheimer and H. Stoecker, Repulsive properties of hadrons in lattice QCD data and neutron stars, [arXiv:2009.10848 [hep-ph]].

25 Y. L. Du, K. Zhou, J. Steinheimer, L. G. Pang, A. Motornenko, H. S. Zong, X. N. Wang and H. Stoecker, Identifying the nature of the QCD transition in heavy-ion collisions with deep learning, [arXiv:2009.03059 [nucl-th]].

24 P. Jakobus, A. Motornenko, R. O. Gomes, J. Steinheimer and H. Stoecker, The possibility of twin star solutions in a model based on lattice QCD thermodynamics, [arXiv:2004.07026 [nucl-th]]

23 - A. Motornenko, J. Steinheimer, V. Vovchenko, S. Schramm and H. Stoecker, QCD equation of state at vanishing and high baryon density: Chiral Mean Field model, [arXiv:2002.01217 [hep-ph]]

22 - Y. L. Du, K. Zhou, J. Steinheimer, L. G. Pang, A. Motornenko, H. S. Zong, X. N. Wang and H. Stöcker, Identifying the nature of the QCD transition in relativistic collision of heavy nuclei with deep learning,

Eur. Phys. J. C 80, no.6, 516 (2020)

21 - L. M. Satarov, I. N. Mishustin, A. Motornenko, V. Vovchenko, M. I. Gorenstein and H. Stoecker, Phase Transitions and Bose-Einstein Condensation in Alpha-Nucleon Matter,

Ukr. J. Phys. 64, no.8, 745-749 (2019)

20 - A. Motornenko, V. Vovchenko, C. Greiner and H. Stoecker, Kinetic freeze-out temperature from yields of short-lived resonances,

Phys. Rev. C 102, no.2, 024909 (2020)

19 M. Hanauske, L. Bovard, E. Most, J. Papenfort, J. Steinheimer, A. Motornenko, V. Vovchenko, V. Dexheimer, S. Schramm, and H. Stoecker, Detecting the Hadron-Quark Phase Transition with Gravitational Waves,

Universe 5, no. 6, 156 (2019)

18 - O. Panova, A. Motornenko, M. I. Gorenstein and H. Stoecker, Backward nucleon production by heavy baryonic resonances in proton-nucleus collisions,

Phys.Rev.C 100 (2019) 5, 054617

17 M. Hanauske, L. Bovard, J. Steinheimer, A. Motornenko, V. Vovchenko, S. Schramm, V. Dexheimer, E. Most, J. Papenfort, and H. Stoecker, MAGIC - how MAtter's extreme phases can be revealed in Gravitational wave observations and in relativistic heavy lon Collision experiments, J. Phys. Conf. Ser. 1271, no. 1, 012023 (2019)

16 A. Motornenko, J. Steinheimer, V. Vovchenko, S. Schramm and H. Stoecker, Matter And Gravitation In Collisions of heavy ions and neutron stars: equation of state,

PoS CORFU2018 (2019) 150

15 - R. Poberezhnyuk, V. Vovchenko, A. Motornenko, M. I. Gorenstein and H. Stoecker, Chemical freezeout conditions and fluctuations of conserved charges in heavy-ion collisions within quantum van der Waals model

Phys. Rev. C 100 (2019) 5, 054904

14 A. Motornenko, J. Steinheimer, V. Vovchenko, S. Schramm and H. Stoecker, Equation of state for hot QCD and compact stars from a mean field approach,

Phys. Rev. C 101 (2020) 3, 034904

13 - K. Taradiy, A. Motornenko, V. Vovchenko, M. I. Gorenstein and H. Stoecker, The analytic structure of thermodynamic systems with repulsive interactions,

https://doi.org/10.1103/PhysRevC.100.065202

12 - L. Csernai, M. Csete, I. Mishustin, A. Motornenko, I. Papp, L. Satarov, H. Stöcker, N. Kroo, Radiation dominated implosion with flat target,

arXiv:1903.10896 [physics.plasm-ph]

11 M. Hanauske, J. Steinheimer, A. Motornenko, V. Vovchenko, L. Bovard, E. Most, J. Papenfort, , S. Schramm, and H. Stoecker, Neutron Star Mergers: Probing the EoS of Hot, Dense Matter by Gravitational Waves,

Particles 2, no. 1, 44 (2019)

10 A. Motornenko, V. V. Begun, V. Vovchenko, M. I. Gorenstein and H. Stoecker, Hadron yields and fluctuations at the CERN Super Proton Synchrotron: system size dependence from $\mathrm{Pb}+\mathrm{Pb}$ to $\mathrm{p}+\mathrm{p}$ collisions,

Phys. Rev. C 99 (2019) 2, 024909

9 - L. M. Satarov, I. N. Mishustin, A. Motornenko, V. Vovchenko, M. I. Gorenstein and H. Stoecker, Phase transitions and Bose-Einstein condensation in alpha-nucleon matter, 
Phys. Rev. C 99, no. 2, 024909 (2019)

8- A. Motornenko, V. Vovchenko, J. Steinheimer, S. Schramm and H. Stoecker, QCD at high density: Equation of state for nuclear collisions and neutron stars,

Nucl. Phys. A 982, 891 (2019)

7 - A. Motornenko, K. Grebieszkow, E. Bratkovskaya, M. I. Gorenstein, M. Bleicher and K. Werner, Eventby-event fluctuations in $\mathrm{p}+\mathrm{p}$ and central $\mathrm{A}+\mathrm{A}$ collisions within relativistic transport models, J. Phys. G 45, no. 11, 115104 (2018)

6 - A. Motornenko, L. Bravina, M. I. Gorenstein, A. G. Magner and E. Zabrodin, Nucleon matter equation of state, particle number fluctuations, and shear viscosity within UrQMD box calculations,

J. Phys. G 45, no. 3, 035101 (2018)

5. V. Vovchenko, A. Motornenko, M. I. Gorenstein and H. Stoecker, Beth-Uhlenbeck approach for repulsive interactions between baryons in a hadron gas,

Phys. Rev. C 97, no. 3, 035202 (2018)

4 V. Vovchenko, A. Motornenko, P. Alba, M. I. Gorenstein, L. M. Satarov and H. Stoecker, Multicomponent van der Waals equation of state: applications in nuclear and hadronic physics, Phys. Rev. C 96, no. 4, 045202 (2017)

3. L. M. Satarov, M. I. Gorenstein, A. Motornenko, V. Vovchenko, I. N. Mishustin, and H. Stoecker, Bose-Einstein condensation and liquid-gas phase transition in alpha-matter,

J. Phys. G 44, 12 (2017)

2. A. Motornenko and M. I. Gorenstein, Cumulative pion production via successive collisions in nuclear medium,

Acta Phys. Polon. Supp. 10, 681 (2017)

1. A. Motornenko and M. I. Gorenstein, Cumulative production of pions by heavy baryonic resonances in proton-nucleus collisions,

J.Phys. G44 (2017) no.2, 025105

\section{VORTRÄGE AUF INTERNATIONALEN KONFERENZEN}

13. Januar 2020, Advances in Astroparticle Physics ANd Cosmology, Saha Institute of Nuclear Physics, Kolkata, Indien

12 - November, 2019, QUARK MATTER 2019, THE XXVIIITH INTERNATIONAL Conference ON UltraRELATIVISTIC NuClEUS-NuCleus Collisions, Wanda Reign Wuhan,

Wuhan, China

11 Oktober, 2019, HIGH ENERGY PHYSICS SEMINAR, Universität Oslo,

Oslo, Norwegen

10 - Mai 2019, NeW TRends In High ENergy Physics,

Odessa, Ukraine

9 - Mai 2019, Nuclear Theory Seminar, Nuclear Science Division, Lawrence Berkeley National Laboratory,

Berkeley, Kalifornien, USA

8 • Januar 2019, COST WORKSHOP ON INTERPLAY OF HARD AND SOFT QCD PROBES FOR COLLECTIVITY IN HEAVYION COLLISIONS, Lund University,

Lund, Schweden

7 - September 2018, Critical Point ANd OnSet of Deconfinement 2018, Corfu Summer Institute, Corfu, Griechenland

6 September 2018, NA61/SHINE Collaboration MeETing, CERN, Genf, Schweiz

5 - Juni 2018, INTERnational SChool of SUbNuClear Physics 56TH Course: from gravitational Waves TO QED, QFD AND QCD, Ettore Majorana Foundation and Centre for Scientific Culture, Erice, Italien

4 - Mai 2017, WorkShop "NuCleAR, PARTICLE PhYSICS AND COSMOLOGY", University of Oslo, Oslo, Norwegen

3• Dezember 2016, Young Scientists Conference "Problems of Theoretical Physics", Bogolyubov Institute for Theoretical Physics,

Kiew Ukraine

2 - Juni 2016, International School of subnuclear Physics 54th Course: The New Physics Frontiers IN THE LHC-2 ERA, Ettore Majorana Foundation and Centre for Scientific Culture,

Erice, Italien

1 - Juni 2016, Critical Point ANd OnSEt of Deconfinement 2016, University of Wroclaw, Breslau, Polen 